\title{
Development of Human Liver Extracellular Matrix Hydrogels for Three Dimensional Cell Culture and Cell Transplantation
}

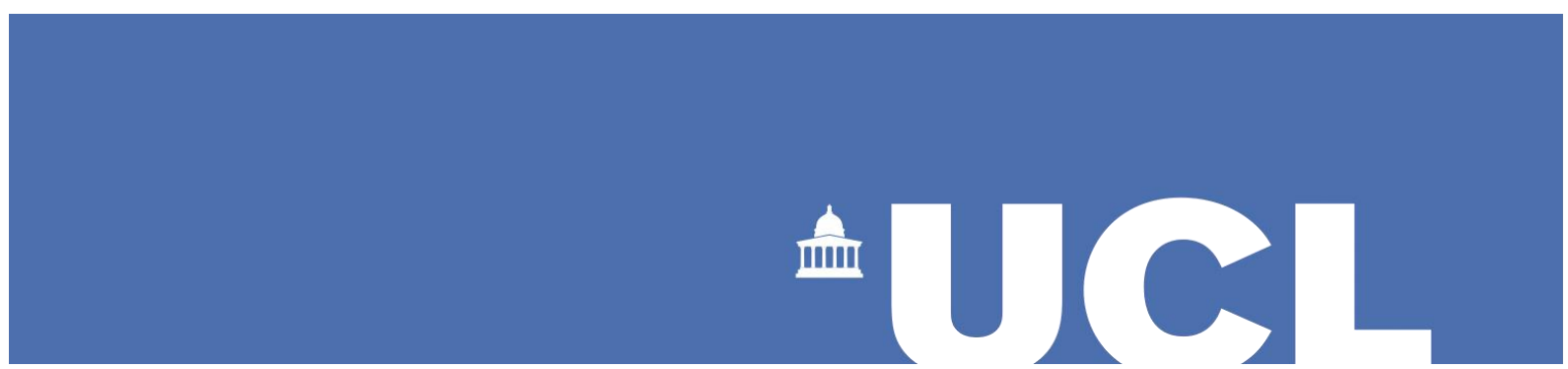

\section{Luca Frenguelli}

Division of Medicine

Institute for Liver and Digestive Health

University College London

A thesis submitted for the degree of

Doctor of Philosophy

Supervisors

Prof. Krista Rombouts

Prof. Massimo Pinzani 


\section{Achievements}

During my $\mathrm{PhD}$ the following achievements were obtained:

\section{Patents:}

- International patent application 62/609467 filed with Engitix Ltd (2017); Frenguelli L, Martinez H, Gatenholm E, Mazza G. "Tissue-specific human bio-inks for the physiological 3D-bio-printing of human tissues for in vitro culture and transplantation".

- UK patent application 1904793.5 filed with UCLB (2019); Mazza G, Frenguelli L, Cecchin D, Caon E, Battaglia G. "Extracellular Matrix Hydrogels".

\section{Publications:}

- Mazza G, Al-Akkad W, Telese A, Longato L, Urbani L, Robinson B, Hall A, Kong K, Frenguelli L, Marrone G, Willacy O, Shaeri M, Burns A, Malago M, Gilbertson J, Rendell N, Moore K, Hughes D, Notingher I, Jell G, Del Rio Hernandez A, De Coppi P, Rombouts K, Pinzani M. Rapid production of human liver scaffolds for functional tissue engineering by high shear stress oscillationdecellularization. Scientific Reports (2017). DOI:10.1038/s41598-017-05134-1

- Mangione P, Mazza G, Gilbertson J, Rendell N, Canetti D, Giorgetti S, Frenguelli L, Curti M, Rezk T, Raimondi S, Pepys M, Hawkins P, Gillmore J, Taylor G, Pinzani $\mathrm{M}$, Bellotti V. Increasing the accuracy of proteomic typing by decellularisation of amyloid tissue biopsies. Journal of proteomics (2017). DOI:10.1016/j.jprot.2017.06.016

- Zhang Z, Xu H, Mazza M, Zhang M, Frenguelli L, Liu Q, Al-Akkad W, Ren J, Zhao $R$, Ren F, Chen X, Huang A, Chen J. Decellularized human liver scaffold-based three-dimensional culture system facilitate hepatitis $B$ virus infection. Journal of Biomedical Materials Research Part A (2019). DOI:10.1002/jbm.a.36690

- Giuffrida P, Curti M, Al-Akkad W, Biel C, Crowley C, Frenguelli L, Telese A, Hall A, Tamburrino D, Spoletini G, Fusai G, Tinozzi FP, Pietrabissa A, Corazza GR, De Coppi P, Pinzani M, Di Sabatino A, Rombouts K, Mazza G. Decellularized Human Gut as a Natural 3D Platform for Research in Intestinal Fibrosis. Inflamm Bowel Dis. 2019. DOl:10.1093/ibd/izz115. 


\section{Poster/ Oral presentations}

- Frenguelli L et al. ECLCB 2016, Ascott. Oral presentation.

- Frenguelli L et al. AASLD 2016 (Boston) The $67^{\text {th }}$ Annual Meeting of the American Association for the Study of Liver Diseases: The Liver Meeting 2016. Oral presentation.

- Frenguelli $L$ et al. APAM 2017 The $11^{\text {th }}$ Annual meeting of the Association of Physicians of Great Britain and Ireland (London). Poster.

- Frenguelli L et al. UCL, Division of Medicine Research Retreat Day 2017 - Annual meeting (London). Oral presentation.

- Frenguelli L et al. EASL 2017 (Amsterdam) The International Liver Congress ${ }^{\mathrm{TM}}$ $2017-52^{\text {th }}$ Annual meeting of the European Association for the Study of the Liver. Oral presentation.

- Frenguelli L et al. EASL 2017 (Amsterdam) The International Liver Congress ${ }^{\mathrm{TM}}$ $2017-52^{\text {th }}$ Annual meeting of the European Association for the Study of the Liver. Poster presentation.

- Frenguelli L et al. BASL 2017 (New Abbot) The Annual meeting of the British Association for the Study of the Liver. Oral presentation.

- Frenguelli L et al. EASL 2018 (Paris) The International Liver Congress ${ }^{\mathrm{TM}} 2017$ $52^{\text {th }}$ Annual meeting of the European Association for the Study of the Liver. Oral presentation.

- Frenguelli L et al. EASL 2018 (Paris) The International Liver Congress ${ }^{\mathrm{TM}} 2017$ $52^{\text {th }}$ Annual meeting of the European Association for the Study of the Liver. Oral presentation.

- Frenguelli L et al. TERMIS World Congress 2018 (Kyoto) The $5^{\text {th }}$ World Congress of the Tissue Engineering and Regenerative Medicine International Society. Oral presentation.

- Frenguelli L et al. TERMIS World Congress 2018 (Kyoto) The $5^{\text {th }}$ World Congress of the Tissue Engineering and Regenerative Medicine International Society. Poster.

- Frenguelli L et al. UCL, Division of Medicine Research Retreat Day 2019 - Annual meeting (London). Oral presentation.

\section{Awards}

- EASL Travel and Registration Bursary Award 2017.

- EASL ILC 2017: Basic Science Seminar, Fellow Speaker Invitation.

- EASL Young Investigator Bursary Award 2018. 


\section{Declaration}

'I, Luca Frenguelli confirm that the work presented in this thesis is my own. Where information has been derived from other sources, I confirm that this has been indicated in the thesis.'

Signed 


\section{Acknowledgment}

I want to thank my supervisors Prof. Krista Rombouts and Prof. Massimo Pinzani for their immense help, patience, and supervision during this project. I'm also very grateful for their effort in reviewing and re-editing this thesis. I would also like to thank $\mathrm{Dr}$ Giuseppe Mazza for his massive effort in coordinating the tissue-engineering projects and for giving me wise advices all the time. Thank you all for supporting and letting me try my own ideas.

I would also like to thank Mr. Andrew Hall and Dr Mustapha Najimi for providing immunohistochemical staining, Dr Claire Crowley for her help with scanning electron microscopy, Dr Federico Scottoni for handling the animal experiment, Prof. Claire Selden and Prof Scot Friedman for providing the hepatic cell lines, Prof. Ludovic Vallier for providing the stem cells and Dr Rute Da Costa Tomaz for helping me with stem cells seeding and immunofluorescence staining. Thanks to all of you for your precious and direct contribution to this project.

I want to say thanks to Walid Al-Akkad, my lab mentor when I joined this amazing group. Thanks for your patience and for the time you invested in explaining and teaching me many skills. Thanks also for being a good friend outside the lab. I want to say thank you to all lab members (and friends of course!) I worked with, in particular Dr ZhenZhen Zang, Dr Marco Curti, Dr Paolo Giuffrida, Dr Domenico Bagordo, Elisabetta Caon, Dr Kessarin Thanapirom, Maria Giovanna Vilia, Simone Canestrari Martina Marrali, David Martos Ruiz, Eric Felli, Lamis Beshir, Yutaka Yasui and Bailin Chen.

I would like to thank my wife Giulia for her unconditional help, trust and support, you are able to transform a very bad day into the best day ever. Now I am realising how many difficulties we faced since we moved to London, but thanks to you everything has been perfect. I could not imagine a better life, and you are the backbone of my entire being. I also want to say thanks to my little princess Sophia, you made every day unpredictable and special, you made our lives incredibly full of happiness. 
Thanks to my mother Elisabetta, my dad Flavio, my brother Andrea and my grandmother Marcella for all the effort you have done to make all of this possible. Without your unconditional love I couldn't even think to move to London, overcome all the problems and start this PhD. Thank you for believing in me and in what I'm doing. 


\section{Table of Contents}

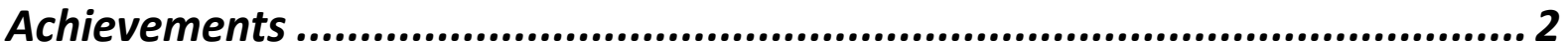

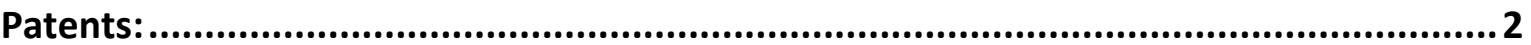

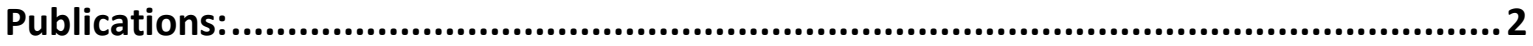

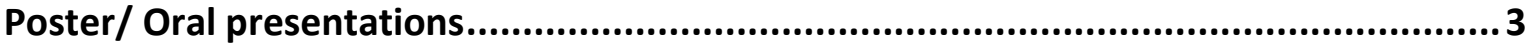

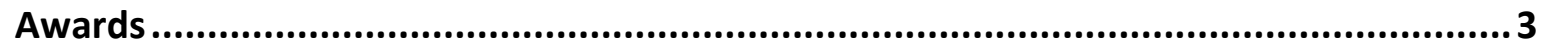

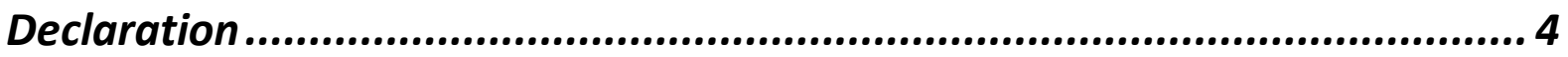

Acknowledgment............................................................................... 5

Table of Figures

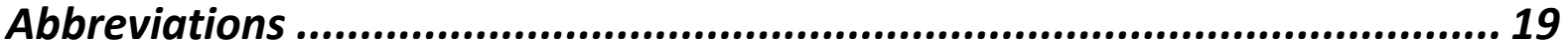

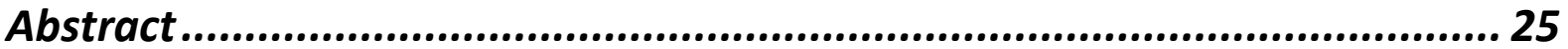

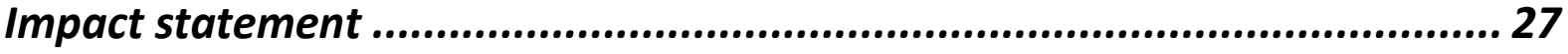

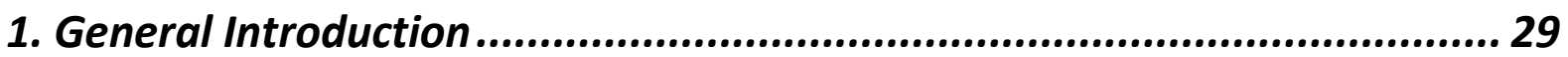

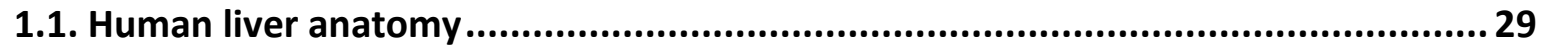

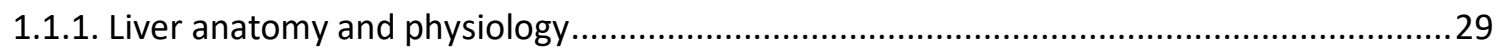

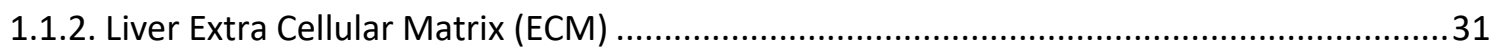

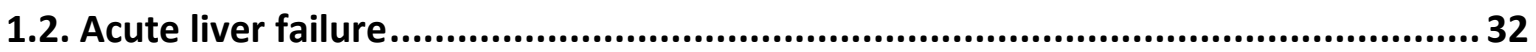

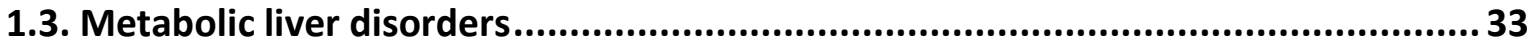

1.4. The treatment of end-stage liver diseases: need for new treatment strategies...... 35

1.4.1. Alternative strategies for the treatment of end-stage liver diseases ................................36

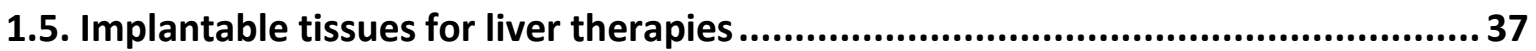

1.6. Current in vitro and in vivo liver disease models ............................................ 41

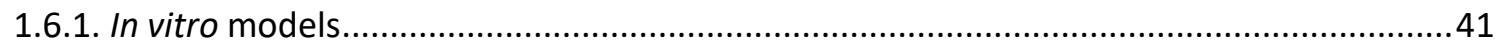

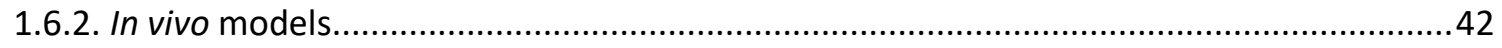


1.7. 2D vs. 3D ECM-based in vitro systems

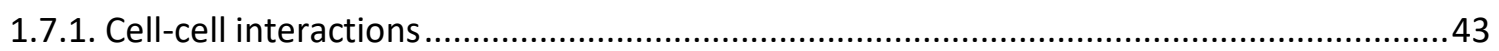

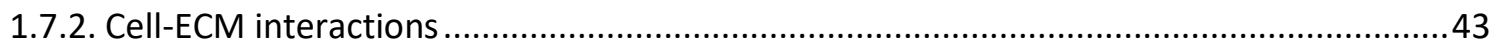

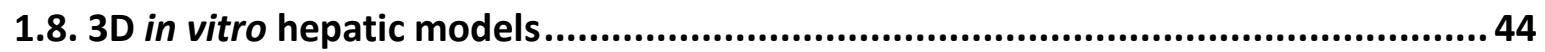

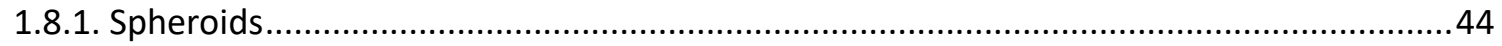

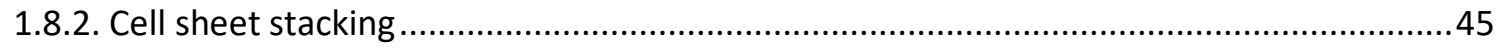

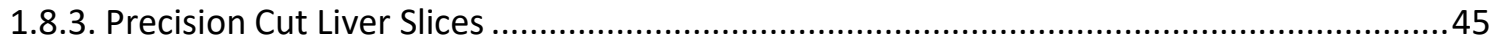

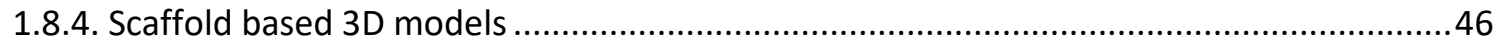

Chapter 2: Development of human liver ECM-hydrogels for liver bioengineering and cell transplantation ................................................ 48

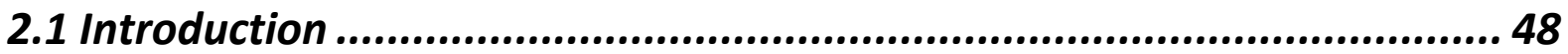

2.1.1 Hydrogels for tissue engineering and regenerative medicine............................. 48

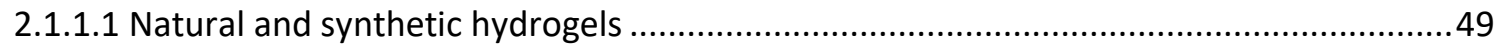

2.1.2 Hydrogel preparation methods............................................................... 50

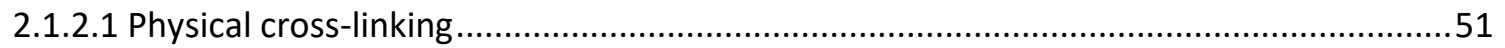

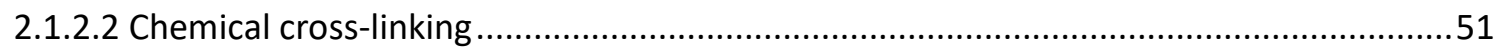

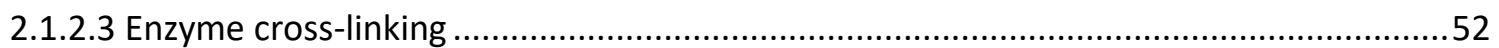

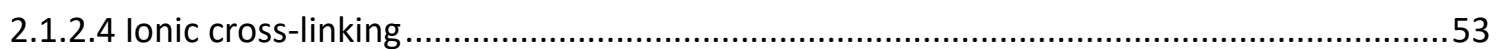

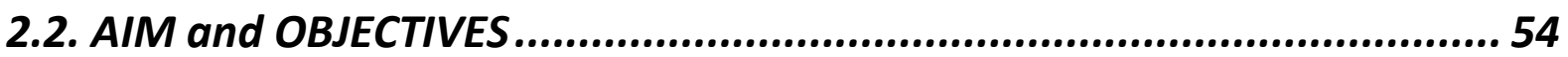

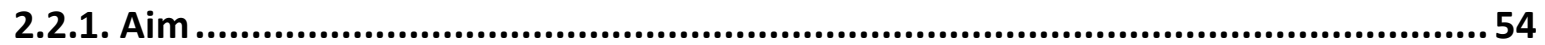

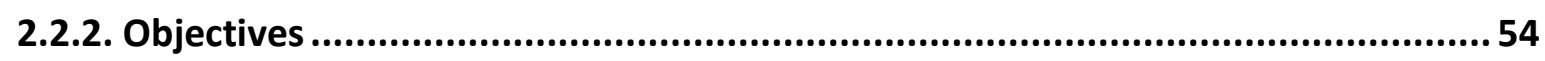

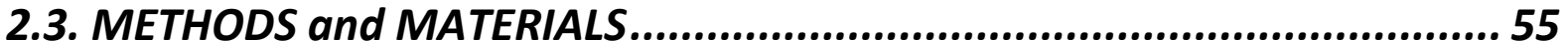

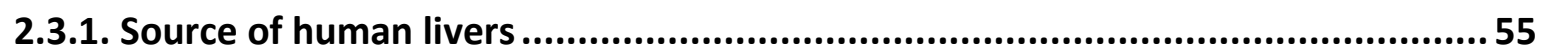

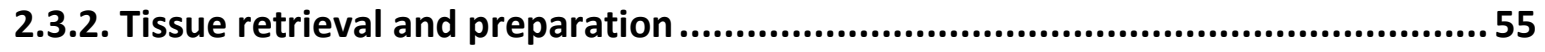

2.3.3. Development of Human Liver ECM-Hydrogels...............................................5 56

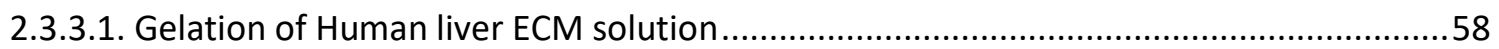

2.3.4. Histology, Immunohistochemistry and Immunofluorescence .............................59

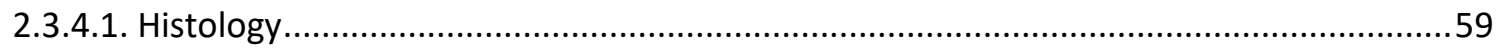




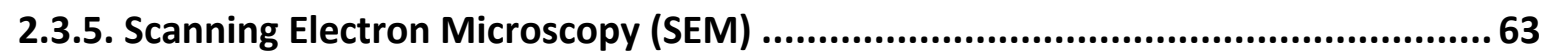

2.3.6. Rheological evaluation of the stiffness of human liver ECM hydrogel .................64

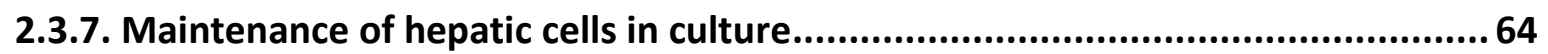

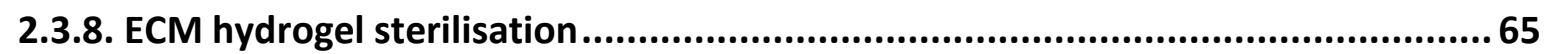

2.3.9. 3D cell cultures in human liver ECM-hydrogels...........................................65

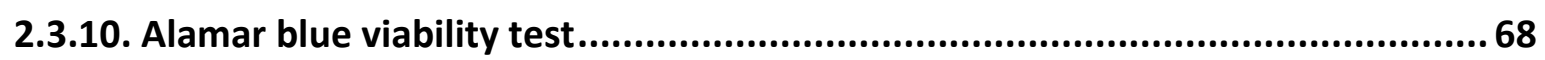

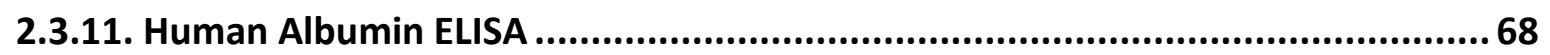

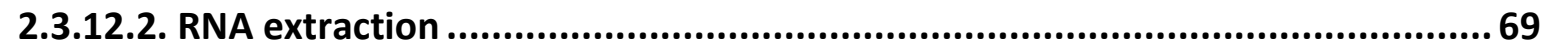

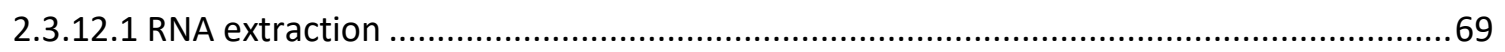

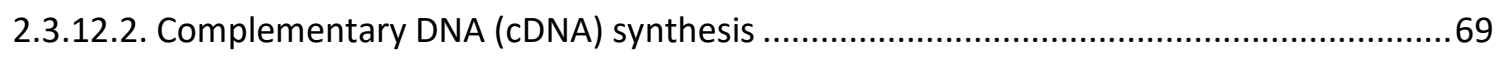

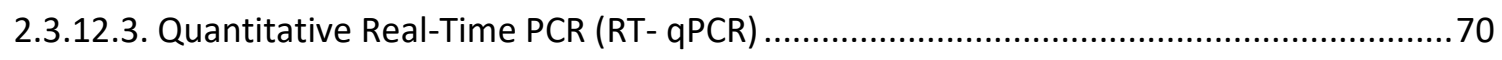

2.3.13. In vivo biocompatibility of bioengineered ECM-hydrogels ........................... 72

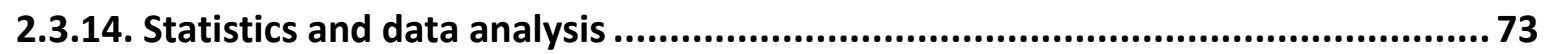

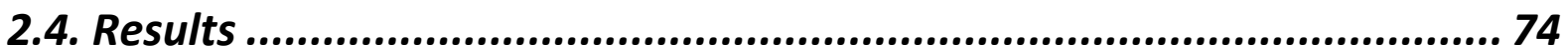

2.4.1. Development of Human Liver ECM-hydrogels ............................................ 74

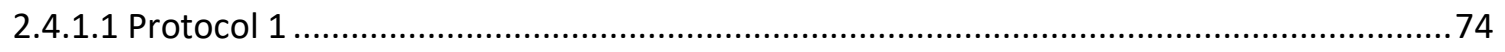

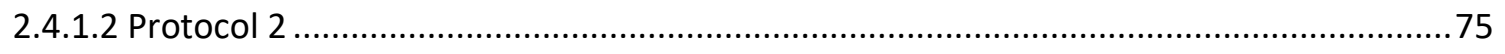

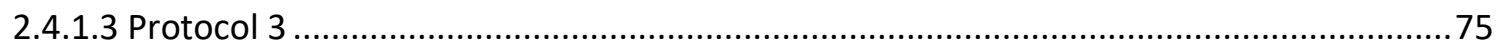

2.4.1.4 Gelation of Human liver ECM solution with gelling agents.........................................76

2.4.2. Characterisation of Human Liver ECM Hydrogel ............................................ 77

2.4.2.1. Histological and Immunohistochemistry analysis of human ECM-hydrogels ..................77

2.4.2.2. Scanning Electron Microscopy (SEM) of human liver ECM-hydrogels...........................78

2.4.2.3. Rheological analysis of human liver ECM-hydrogels................................................... 79

2.4.3. Assessing the in vitro biocompatibility of human liver ECM-hydrogels .............. 80

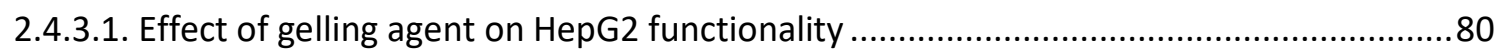

2.4.3.2. Evaluation of the importance of ECM proteins for cell attachment, viability and

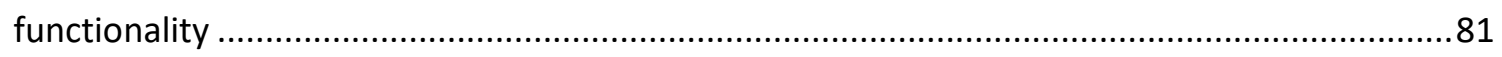


2.4.4 Comparison in gene expression and functionality of HepG2 cultured in ECMhydrogels and in acellular liver cubes

2.4.6 Evaluation of Human Induced Pluripotent Stem Cells (IPSC) differentiation on ECMhydrogels.

2.5. Discussion 96

2.5.1 Advantages of using human livers unsuitable for transplantation ...................................96

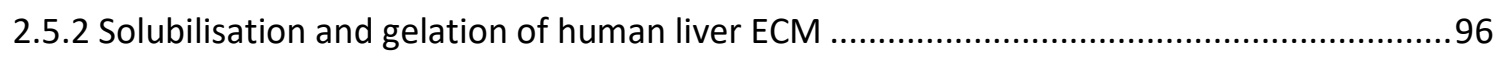

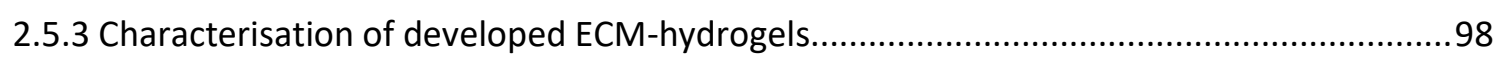

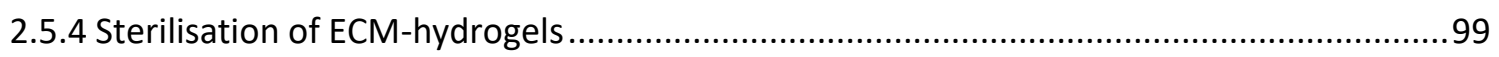

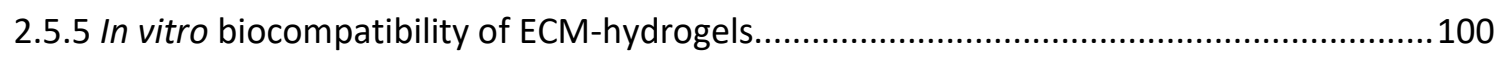

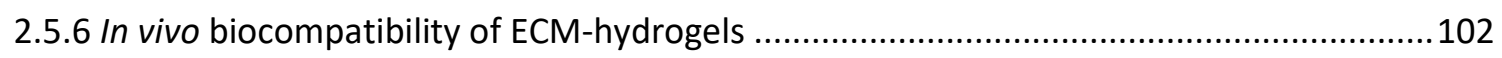

2.5.7 IPSC differentiation towards hepatocyte like cells..........................................................103

Chapter 3: 3D Bio-printing of Human Hepatic tissue using Human Liver Extracellular Matrix as Tissue-Specific Bio-ink........................................ 105

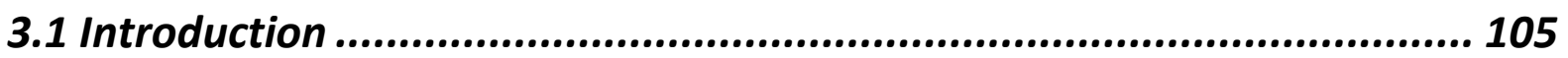

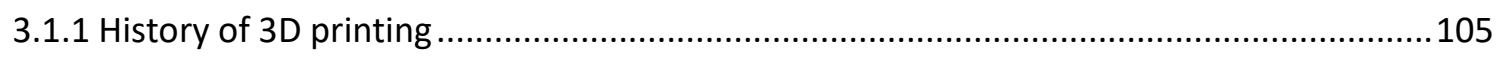

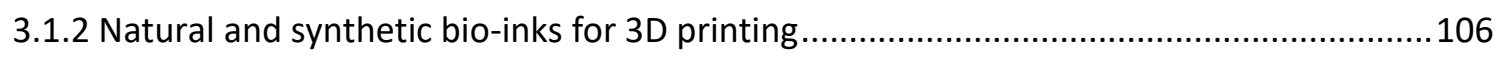

3.3.3 Decellularized extracellular matrix as a new source of bio-inks ......................................108

3.2 Aim and Objectives ........................................................................ 110

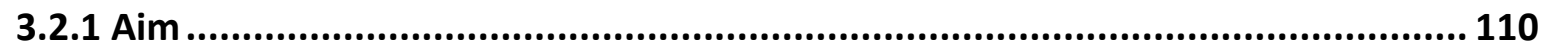

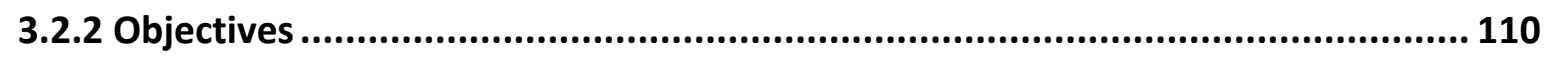

3.3 Methods and Materials .................................................................. 111

3.3.1 Development of Human Liver ECM solution ........................................... 111

3.3.2 Maintenance of hepatic cells in culture.................................................... 111

3.3.3 Printability tests of human liver ECM solution combined with Cellink bio-ink at different ratios 


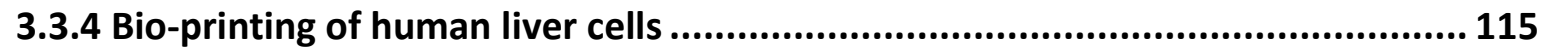

3.3.5 Cell culture in 3D bio-printed samples ................................................. 118

3.3.6 Live/Dead cell double staining ............................................................ 119

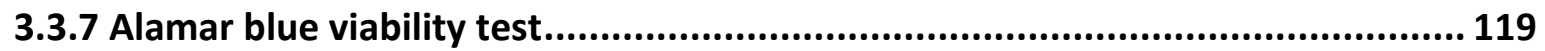

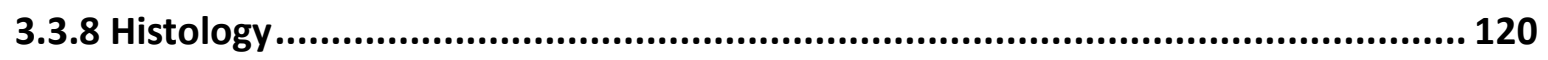

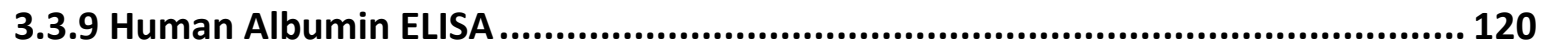

3.3.10 Lactate Dehydrogenase (LDH) Cytotoxicity Assay ....................................... 121

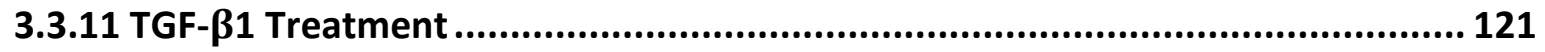

3.3.12 Human Pro-Collagen 1 alpha 1 ELISA................................................... 122

3.3.13 RNA extraction, reverse transcription and gene expression analysis.............. 122

3.3.13.1 RNA extraction ................................................................................... 122

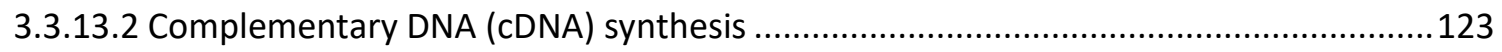

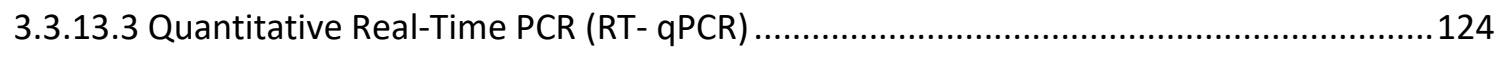

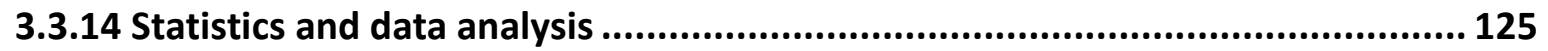

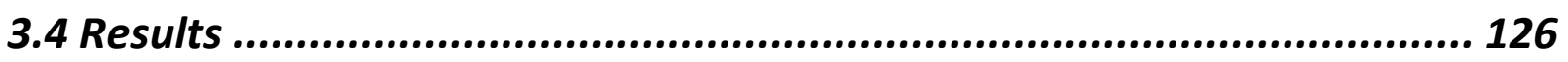

3.4.1 Assessing printability of human liver ECM solution combined with Cellink bio-ink at

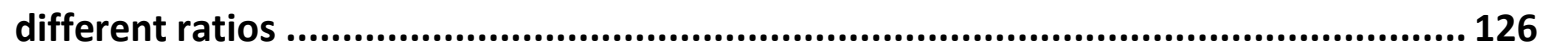

3.4.1.1 Bio-printing of liver ECM solution:Cellink in ratio 20:80........................................126

3.4.1.2 Bio-printing of liver ECM solution:Cellink in ratio 30:70 ...................................128

3.4.1.3 Bio-printing of liver ECM solution:Cellink in ratio 50:50......................................130

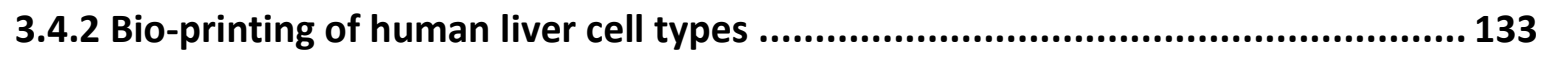

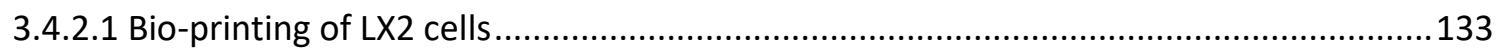

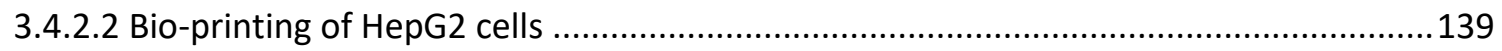

3.4.2.3 Bio-printing of primary human hepatocytes.................................................. 145

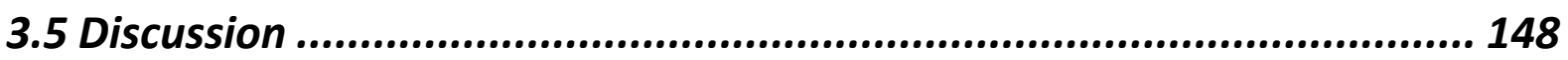


3.5.1 Advantages of ECM+Cellink bio-ink compared to available bio-inks .

3.5.2 Printability tests using ECM+Cellink bio-ink ..................................................... 150

3.5.3 In vitro biocompatibility of ECM+Cellink bio-ink .................................................... 150

3.5.4 ECM+Cellink bio-ink vs ECM-Agarose hydrogels .................................................. 152

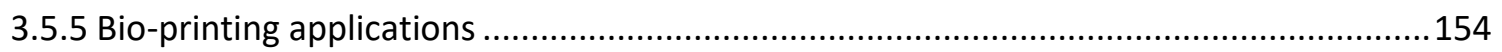

Chapter 4: Hep-Gel, a novel in vitro 3D model for the study of Hepatocellular carcinoma (HCC) and drug screening tests .............................................. 156

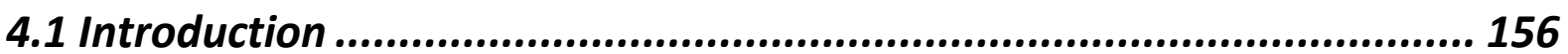

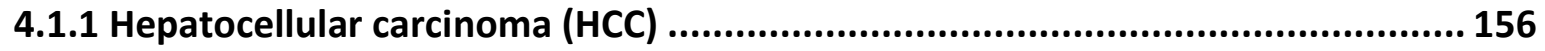

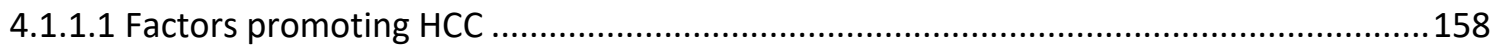

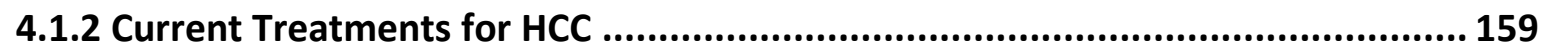

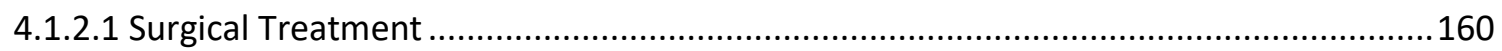

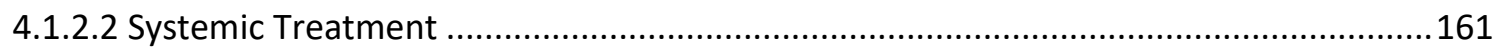

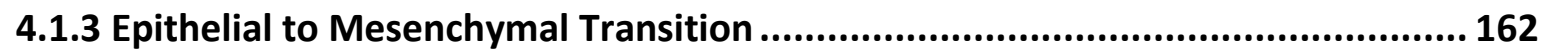

4.1.4 Hepatocellular Carcinoma SNU and HepG2 Cell Lines..................................... 164

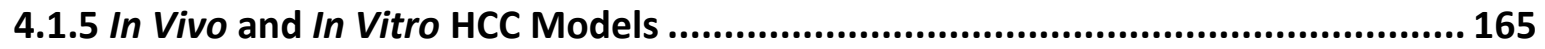

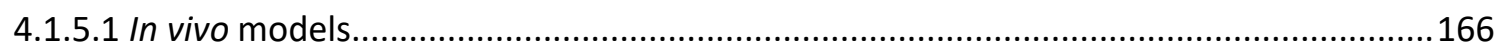

4.1.5.2 In vitro Models ...................................................................................... 167

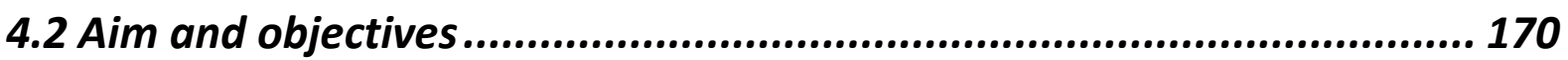

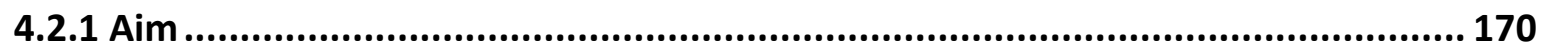

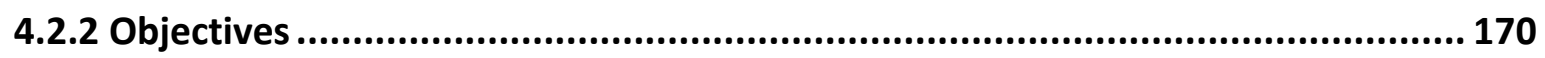

4.3 Methods and Materials.................................................................... 171

4.3.1 Preparation of Human Liver ECM solution ................................................... 171

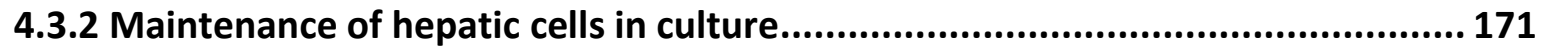

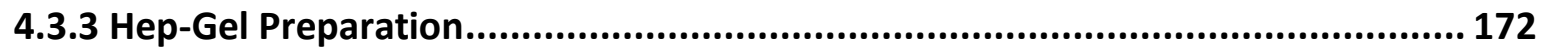

4.3.4 3D determining the optimal cell density in Hep-Gel ....................................... 172

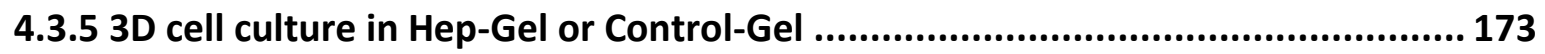




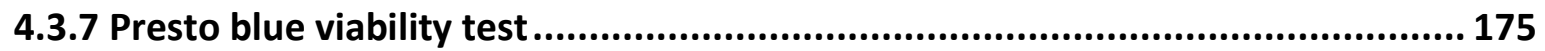

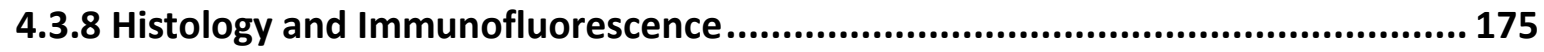

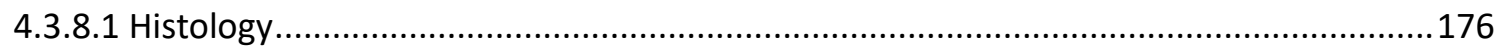

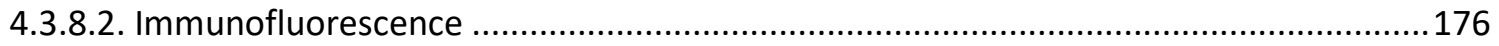

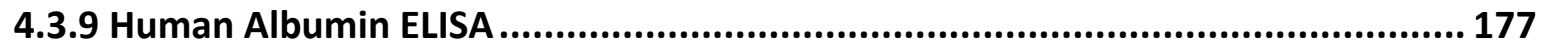

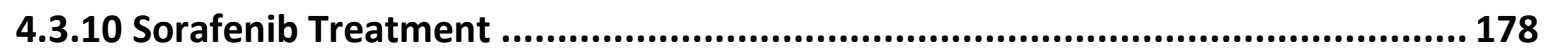

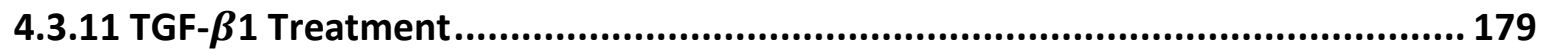

4.3.12 RNA extraction, reverse transcription and gene expression analysis............... 179

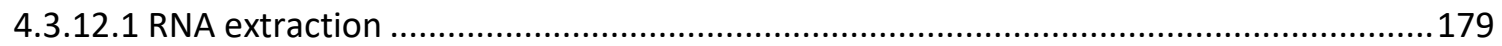

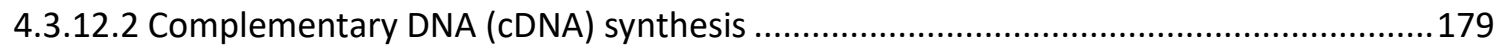

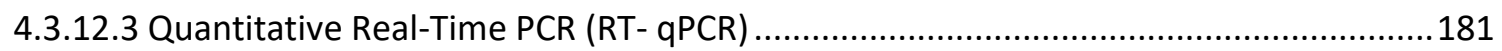

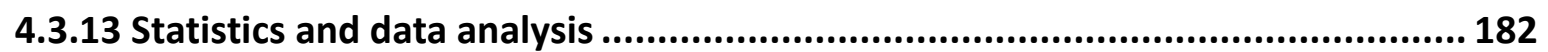

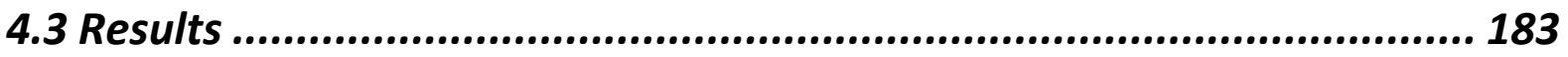

Assessing the optimal HepG2 cell density in Hep-Gel........................................... 183

4.3.2 Assessing the in vitro biocompatibility of the Hep-Gel ................................... 185

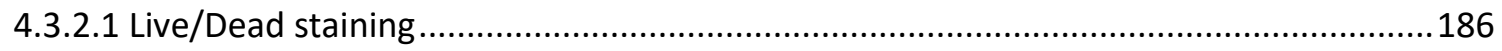

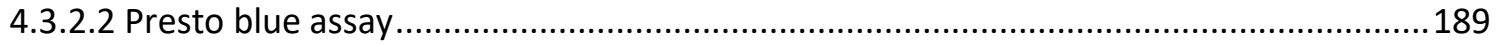

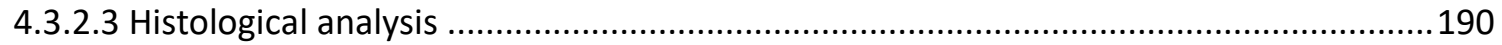

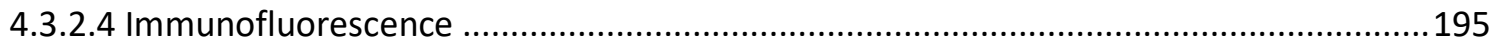

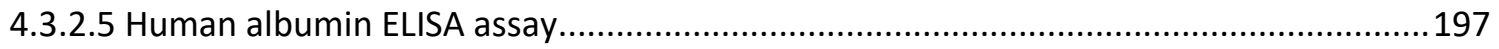

In-vitro Hepatocellular Carcinoma (HCC) models ................................................. 198

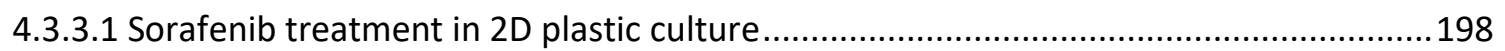

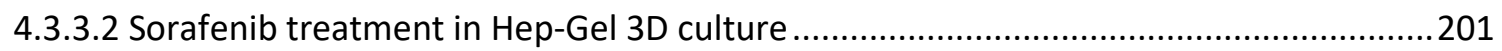

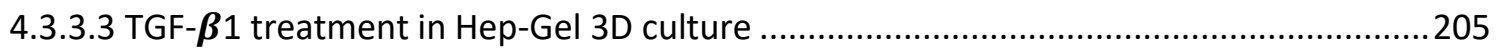

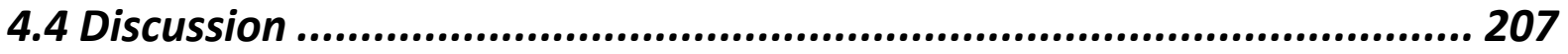

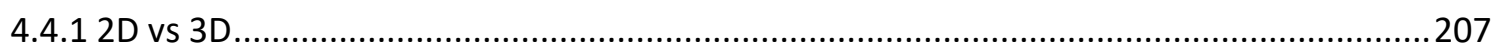

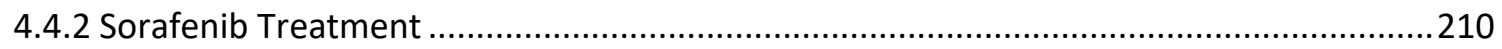

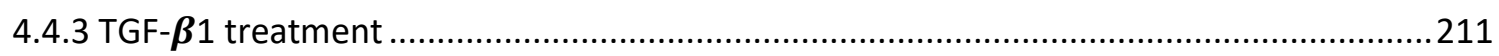


Chapter 5: General discussion and future work .................................... 213

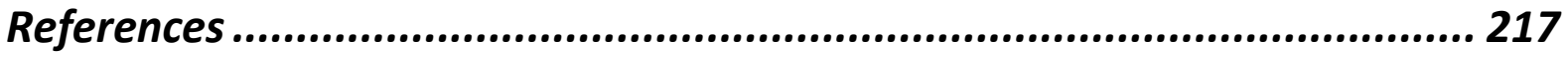




\section{Table of Figures}

Figure 1. Anatomical division of the human liver................................................ 29

Figure 2. Hepatic cells organisation in the human liver lobules............................. 31

Figure 3. Representation of liver sinusoid in health and diseased liver................... 32

Figure 4. Tissue engineering strategies for the treatment of liver disease. .............. 40

Figure 5. Schematic comparing effect of $2 D$ and $3 D$ cultures on cells................. 44

Figure 6. Histology of porcine liver and human healthy liver .................................5 50

Figure 7. Schematic view of the protocols carried out to prepare the ECM liver

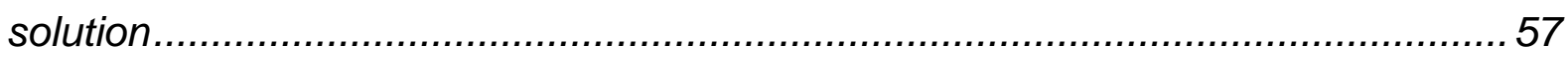

Figure 8. Schematic view of the process for producing hydrogels.........................59

Figure 9. Schematic representation of the timeline for 3D cell culture. ...................66

Figure 10. Schematic representation of IPSCs seeded on ECM-hydrogels. ............67

Figure 11. Schematic overview for the surgical implantation in mice of HepG2

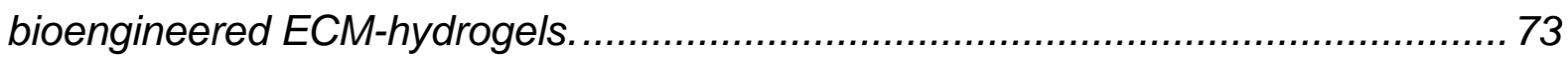

Figure 12. Outcome of Protocol 1 for the development of ECM-hydrogels ............. 74

Figure 13. Outcome of Protocol 2 for the development of ECM-hydrogels. ............ 75

Figure 14. Outcome of Protocol 3 for the development of ECM-hydrogels. ............. 76

Figure 15. Macroscopic view of ECM-hydrogels. ......................................... 77

Figure 16. Histological and Immunohistological characterisation ECM-hydrogels ... 78

Figure 17. Scanning Electron Microscopy (SEM) of ECM-hydrogels ..................... 79

Figure 18. Rheological characterisation of ECM hydrogels................................. 80

Figure 19. Albumin ELISA from HepG2 bioengineered ECM-hydrogels. ................ 81

Figure 20. H\&E staining, immunoflorescence and albumin ELISA from bioengineered HepG2 ECM-hydrogels and Agarose only hydrogels.

Figure 21. Alamar blue and albumin ELISA from bioengineered HepG2 ECM-

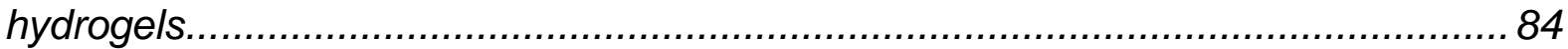

Figure 22 Comparison between ECM-hydrogels and acellular liver cubes ............. 85

Figure 23. Surgical procedure for the implantation of bioengineered HepG2 ECM-

hydrogels into the omentum of immunodeficient mice.

Figure 24. Surgical procedure for the removal of bioengineered HepG2 ECM-

hydrogels from the omentum of immunodeficient mice..... 88 
Figure 25. H\&E staining of bioengineered ECM-hydrogels with HepG2 implanted for three weeks into the omentum of immunodeficient mice.

Figure 26. mmunohistochemistry staining for human Ku80 of bioengineered ECMhydrogels with HepG2 implanted into the omentum of immunodeficient mice. 90 Figure 27. Albumin secretion analysis from iPSC cultured on 3D ECM-hydrogels... 92 Figure 28. Gene analysis of IPSC cultured on ECM-hydrogels. 93

Figure 29. Immunofluorescent images of IPSC cultured in ECM-hydrogels and on plastic. 94

Figure 30. Schematic view of performed bio-printed tests. 113

Figure 31. Line deposition test of the ECM+Cellink bio-ink in ratio 20:80............. 127

Figure 32. Spiral test using ECM+Cellink bio-ink in ratio 20:80........................ 127

Figure 33. Bio-printing of a cube using ECM+Cellink bio-ink in ratio 20:80........... 128

Figure 34. Line deposition test of the ECM+Cellink bio-ink in ratio 30:70.............. 129

Figure 35. Spiral test using ECM+Cellink bio-ink in ratio 30:70......................... 129

Figure 36. Bio-printing of a cube using ECM+Cellink bio-ink in ratio 30:70........... 130

Figure 37. Line deposition test of the ECM+Cellink bio-ink in ratio 50:50 ............ 131

Figure 38. Spiral test using ECM+Cellink bio-ink in ratio 50:50 .......................... 132

Figure 39. Bio-printing of a cube using ECM+Cellink bio-ink in ratio 50:50 ........... 132

Figure 40. Live/Dead staining of bio-printed LX2 at 7 and 13 days of cell culture. 134 Figure 41. Alamar blue of bio-printed LX2 after 13 days of cell culture................. 135

Figure 42. H\&E staining of bio-printed LX2 after 13 days of cell culture. .............. 136 Figure 43. Extracted mRNA from bio-printed LX2 bioprinted after 7 and 13 days of cell culture.

Figure 44. Gene analysis of bio-printed LX2 after 7 and 13 days of cell culture, with or without exposure to TGF- $\beta 1$ 138

Figure 45. ELISA Pro-Collagen 1 secretion from bio-printed LX2 samples........... 139

Figure 46. Live/Dead staining of bio-printed HepG2 samples. ............................. 140

Figure 47. Alamar blue of bio-printed HepG2 after 13 days of cell culture ............. 141

Figure 48. H\&E staining of bio-printed HepG2 after 13 days of cell culture............ 142

Figure 49. Extracted mRNA from HepG2 bio-printed samples............................ 143

Figure 50. Gene analysis of bio-printed HepG2 samples.................................. 144

Figure 51. ELISA Albumin secretion from bio-printed HepG2 samples................. 145

Figure 52. Live/Dead staining of bio-printed human hepatocytes samples ............ 146 
Figure 53. Lactate Dehydrogenase and Albumin ELISA from bio-printed hepatocytes samples.

Figure 54. UK mortality rate data since 1970

Figure 55. Schematic view of HCC development following the progression of fibrosis towards cirrhosis fibrosis 159

Figure 56. Schematic view of EMT/MET processes. 164

Figure 57. Schematic representation of the timeline for 3D cell culture in Hep-Gel or Control-Gel..

Figure 58. Live/Dead staining of HepG2 embedded in Hep-Gel at different cell density and cultured for 7 days. 184

Figure 59. Live/Dead staining of HepG2 embedded in Hep-Gel at different cell density and cultured for 13 days.. 185

Figure 60. Live/Dead staining of HepG2 cultured in Hep-Gel and Control-Gel. 189 Figure 61. Presto blue assay of HepG2 cells cultured in Hep-Gel and Control-Gel at day 1, 7 and day 13 of cell culture. 190

Figure 62. Histology of HepG2 cells cultured for 7 days in Control-Gel. ............... 192 Figure 63. Histology of HepG2 cells cultured for 13 days in Control-Gel .............. 193 Figure 64. Histology of HepG2 cells cultured for 7 days in in Hep-Gel.................. 194 Figure 65. Histology of HepG2 cells cultured for 13 days in in Hep-Gel.. 195 Figure 66. Immunofluorsecenc DAPI and Phalloidin staining of HepG2 cells cultured for 13 days in Hep-Gel and Control-Gel. 196 Figure 67. Immunoluorescence EpCAM and DAPI staining of HepG2 cultured for 13 days on Hep-Gel.

Figure 68. Albumin ELISA from bioengineered HepG2 Hep-Gel and Control-Gel after 13 days of cell culture. 198

Figure 69. Bright-field microscopy images and presto blue assay of 2D HepG2 control and treated samples with Sorafenib.

Figure 70. Bright-field microscopy images and presto blue assay of 2D SNU-449 control and treated samples with Sorafenib.

Figure 71. Live/Dead images and presto blue of bioengineered HepG2 Hep-Gel treated with different doses of Sorafenib. 202 Figure 72. Live/Dead images and presto blue of bioengineered SNU-449 Hep-Gel treated with different doses of Sorafenib. 204 
Figure 73. Live/Dead staining of bioengineered SNU-449 Hep-Gel treated with TGF$\beta 1 .$. 205

Figure 74. Gene expression of bioengineered SNU-449 Hep-Gel treated samples with TGF- $\beta 1$. 206 


\section{Abbreviations}

2-AAF - 2-acetylaminofluorene

AAT - alpha 1 antitrypsin

ACTA2 - $\alpha$-smooth muscle actin 2

AFP - alpha feto protein

AGT - alanine-glyoxylate amino-transferase

ALB - albumin

$\alpha$-SMA - alpha-smooth muscle actin

ATCC - American type culture collection

ATP7B - ATPase copper transporting beta

$\mathrm{BAL}$ - bio-artificial liver devices

BSA - bovine serum albumin

$\mathrm{CaCl}_{2}$ - calcium chloride

CAFs - cancer associated fibroblasts

CK - Cytokine

CN - Crigler-Najjar

$\mathrm{CO}_{2}$ - carbon dioxide

COL1A1 - collagen type 1

CPS - carbamyl phosphate synthetase

CLD - chronic liver diseases 
CYP1A2 - cytochrome P450 family 1 subfamily A member 2

DAPI - 4',6-diamidino-2-phenylindole

DEN - diethylnitrosamine

DMEM- dulbecco's modified eagle medium

2D- two-dimensional

3D - three-dimensional

dECM - decellularized extracellular matrix

$\mathrm{dH}_{2} \mathrm{O}$ - distilled water

DILI - drug-induced liver injury

ECM - extra-cellular matrix

EGF - epidermal growth factor

EGFR - epidermal growth factor receptor

EMT - epithelial to mesenchymal transition

EpCAM - epithelial cell adhesion molecule

EVG - elastic Van Gieson

FBS - foetal bovine serum

FDA - fluorescein diacetate

FGFR- fibroblast growth factor receptor

GAGs - glycosaminoglycans

GAPDH - glyceraldehyde 3-phosphate dehydrogenase

GMP - good manufacturing practices 
GF - growth factors

GSD - glycogen storage disease

HA - hyaluronic acid

HBSS - Hanks balanced salt solution

HBV - hepatitis B virus

HCV - hepatitis C virus

HCC - hepatocellular carcinoma

$\mathrm{HCl}$ - hydrochloric acid

H\&E - haematoxylin \& eosin

HGF - hepatocyte growth factor

$\mathrm{HH}$ - hereditary hemochromatosis

HNF4A - hepatocyte nuclear factor 4 alpha

HRP - horseradish peroxidase

HSC - hepatic stellate cells

HTS - high throughput screening

IPSCs - induced pluripotent stem cells

IDA - industrial denatured alcohol

IL-6 - interleukin-6

LOX- lysyl oxidase

IPSCs - induced pluripotent stem cells

IMS - industrially methylated spirits 
kPa - kilo Pascal

LDH - lactate dehydrogenase

LSEC - liver sinusoidal endothelial cells

MARS - molecular adsorbent recirculating system

MEK - mitogen-activated protein kinase/extracellular signal-regulated kinase kinase

MEKK - MAPK/Erk kinase kinase

MEM - minimum essential medium

MET - mesenchymal to epithelial transition

$\mu \mathrm{l}$ - microliter

$\mu \mathrm{M}$ - micromolar

$\mathrm{ml}$ - millilitre

NAD+ - nicotinamide adenine dinucleotide

$\mathrm{NADH}$ - nicotinamide adenine dinucleotide hydrogen

$\mathrm{NaOH}$ - sodium hydroxide

$\mathrm{NASH}$ - non-alcoholic steatohepatitis

NOD/SCID - non-obese diabetic/severe combined immunodeficiency

NOS - reactive nitrogen species

$\mathrm{NR} 1 \mathrm{H} 4$ - nuclear receptor subfamily 1 group $\mathrm{H}$ member 4

NPCs - non-parenchymal cells

NTP - national toxicology program

$\mathrm{OSO}_{4}$ - osmium tetroxide 
OTC - ornithine transcarbamylase

PBS - phosphate buffered saline

PDGF - platelet derived growth factor

PDGFR- $\beta$ - platelet-derived growth factor receptor-beta

PEG - poly-ethylene glycol

PI - propidium iodide

PIPAAm - poly (N-isoproplyacrylamide)

PLA - poly (I)-lactic acid

PCL - poly-caprolactone

PEGDA - poly (ethylene glycol)-diacrylate

PEGDMA - poly (ethylene glycol)-dimethacrylate

RAF- rapidly accelerated fibrosarcoma

ROS - reactive oxygen species

RT- qPCR - quantitative real-time polymerase chain reaction

SEM - scanning electron microscopy

SMAD - suppressor of mothers against decapentaplegic

SNU - Seoul national university

SPAD - single pass albumin dialysis

SR - picro-sirius red

TAA - thioacetamide 
TAECs - tumour associated endothelial cells

TBS - tris buffered saline

TNF - tumour necrosis factor

TTI - tyrosinemia type I

UGT1A1 - uridine diphosphate glucuronosyltransferase 1 family, polypeptide A1 VEGFR - vascular endothelial growth factor receptor

w/v - weight/volume 


\section{Abstract}

Introduction: It is increasingly evident that the currently available in vivo and in vitro methodologies for disease modelling are sub-optimal in recapitulating the complexity of human pathophysiology, as confirmed by the high failure rate of drug candidates due to lack of efficacy and safety. Moreover, hepatocyte transplantation has been tested as an alternative to liver transplantation for the treatment of liver diseases, but its applicability is hampered by the limited source of hepatocytes and poor hepatocyte engraftment.

Aims: to develop human liver ECM hydrogels as novel in vitro platform for target identification/drug screening and for cell transplantation.

Methods: Human livers unsuitable for transplantation were decellularized. The resulting ECM scaffold was then lyophilized and the resultant liver ECM powder was solubilised and mixed with three different biomaterials such as agarose, inert bio-ink or a synthetic thermo-responsive copolymer for hydrogel development. Samples were bioengineered with human hepatic cell lines (HepG2, LX2 or SNU-449), stem cells (IPSCs) or human primary hepatocytes. Validation of the hepatocellular carcinoma (HCC) model was investigated through treatment of SNU-449 samples with Sorafenib and TGF- $\beta 1$. Furthermore, HepG2 bioengineered hydrogels were implanted for 3 weeks in immune-deficient mice. Samples were analysed by histology, immunofluorescence, immunohistochemistry, viability assays, gene expression and metabolic activity.

Results: Bioengineered human liver ECM-based hydrogels with human liver cells showed an increase in cell survival, engraftment, proliferation and functionality compared to agarose, inert bio-ink or synthetic thermo-responsive copolymer. Viability assays of SNU-499 cells, upon Sorafenib treatment, revealed differences between 2D and 3D modelling in HCC. Implanted HepG2 ECM-hydrogels, retrieved from mice, showed that cells were still alive and engrafted. In vitro, ECM hydrogels combined with synthetic thermo-responsive copolymer showed the highest cell viability, better 
reproducibility, required less ECM volume and a smaller number of cells compared to ECM hydrogels combined with agarose or inert bio-ink.

Conclusion: This study describes the development and the technical validation of human liver ECM hydrogels for in vitro and in vivo applications. 


\section{Impact statement}

In the United Kingdom, the incidence rate of liver disease is dramatically increased since 1970 and it is considered the fifth 'big killer' in England \& Wales. Liver disease due to viral infection, alcohol, and metabolic causes is currently responsible for around $4 \%$ of all deaths worldwide.

At the moment, liver transplantation is the only effective treatment for advanced liver diseases but is limited by high costs and deficiency in donor organs and $15 \%$ to $25 \%$ of patients die while waiting for liver transplantation.

To overcome these problems, the transplantation of hepatocytes has been tested as an alternative therapy to orthotropic liver transplantation for the treatment of acute liver failure and metabolic liver deficiencies. The successful rate of this therapy is limited by shortage of available hepatocytes and poor hepatocyte engraftment.

Furthermore, currently available in vivo and in vitro models are inefficient to recapitulate the complexity of human pathophysiology, as confirmed by the high failure rate of drug candidates during clinical trials. To date, the majority of in vitro experiments in the drug discovery and development process are performed using conventional 2D plastic surfaces which have limitations as studies demonstrated that cells do not behave in vivo-like when cultured on rigid plastic surfaces. This underlines the need of an effective in vitro liver model for new target identification and drug screening processes.

The development of induced pluripotent stem cells (IPSCs) holds great promise for the realization of a personalized regenerative medicine and for the development of in vitro disease models. Important, IPSCs differentiate towards hepatocyte-like cells and could therefore replace primary hepatocytes for both in vitro and in vivo applications and overcome the limitation in cell availability. Indeed, IPSCs cells can be derived directly from the patient, can be differentiated into a variety of adult somatic cells including hepatocytes, and thus used for autologous transplantation in order to restore the functionality of the damaged organs without the need of an immunosuppressive therapy. Alternatively, IPSCs cells can be used to develop in vitro models to study the 
mechanisms underlying human diseases, to study tissue-and organ regeneration as well as for screening of new drugs.

So far, the major limitation of using IPSCs as replacement of human primary hepatocytes is their blunted phenotype when compare to the primary cells.

I believe that human liver ECM hydrogels, presented in this study, provide a more physiological microenvironment for hepatic cells allowing them to develop a phenotype more faithfully representing their effective function in vivo. Thus, human liver ECM hydrogels can be used to replace the conventional 2D plastic culture systems used for target identification and drug screening processes.

In future, human liver ECM hydrogels could be employed in clinic to improve long-term engraftment of primary hepatocytes or IPSC differentiated towards hepatocytes-like cells. Hence, ECM hydrogels bioengineered with human hepatocytes or IPSCs can be used as a treatment for metabolic disorder and acute liver failure, or function as a bridging strategy for whole organ transplantation. 


\section{General Introduction}

\subsection{Human liver anatomy}

\subsubsection{Liver anatomy and physiology}

The liver, not considering the skin, is the largest and the heaviest organ in the human body, accounting for $2-3 \%$ of the body weight [1]. It is characterised by a conical shape and is located in the upper right portion of the abdominal cavity [2].

The liver is covered by a thin layer of connective tissue called Glisson's capsule [3] and can be anatomically divided in 8 segments or four lobes, right and left lobes, divided by the falciform ligament, caudate and quadrate lobes (Figure 1) [4].

a

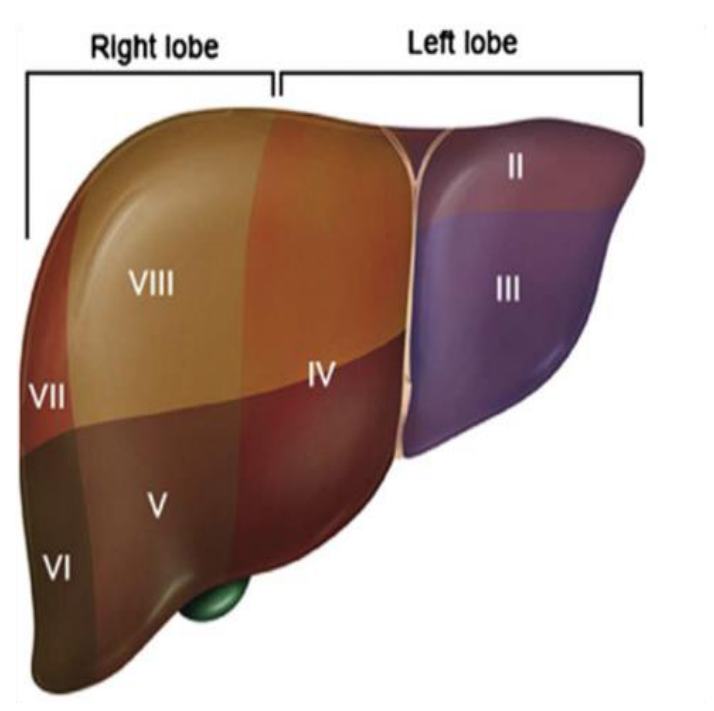

b

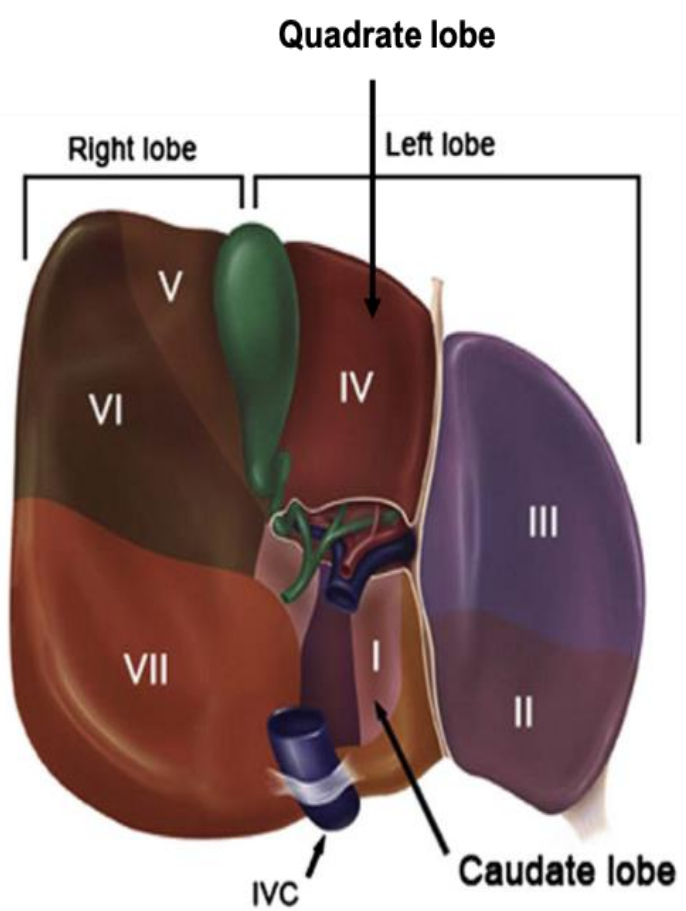

Figure 1. Anatomical division of the (a) anterior and (b) posterior faces of the human liver in lobes and segments. Images modified from S. R. Z. Abdel-Misih et al 2014 [1]. 
Liver lobes and segments can be further divided in lobules, the functional units of the liver characterised by a hexagonal shape and primarily composed of hepatocytes disposed radially around the central vein (Figure 2). Hepatocytes are parenchymal cells representing approximately $80 \%$ of the entire liver mass and perform most of the hepatic functions, as they play a crucial role in the metabolism of carbohydrate, lipids, and proteins, blood filtration and chemical detoxification, storage of glycogen, lipids and minerals, production of numerus vital molecules including blood-clotting proteins, albumin and bile [5]. The remaining $20 \%$ is composed of different non-parenchymal cells (NCPs) such as Hepatic Stellate Cells (HSC), Liver Sinusoidal Endothelial Cells (LSECs) and Kupffer cells [6]. Between the cords of hepatocytes are located the sinusoids, the microvascular unit of the liver characterised by abundant fenestrations made by the sinusoidal endothelial cells (LSECs) ideal to allow the bidirectional exchange of molecules between blood and hepatocytes [7]. Kupffer cells are liver tissue macrophages located in the lumen of the liver sinusoids that play an important role in digesting foreign antigens, bacteria fungi and parasites derived from the gut [8]. Hepatic stellate cells, liver specific pericytes, are located in the space of Disse that divides the hepatocytes from the sinusoidal endothelium. HSC store lipids and Vitamin A and play an essential role in the physiology of the space of Disse [9]. Indeed, in response to liver injury, these cells change from a quiescent state into an activated phenotype that leads to and increased secretion of extracellular matrix (ECM) [10], and for this reason they are considered the key cellular effectors of liver fibrogenesis in response to chronic liver injury [11-13]. 


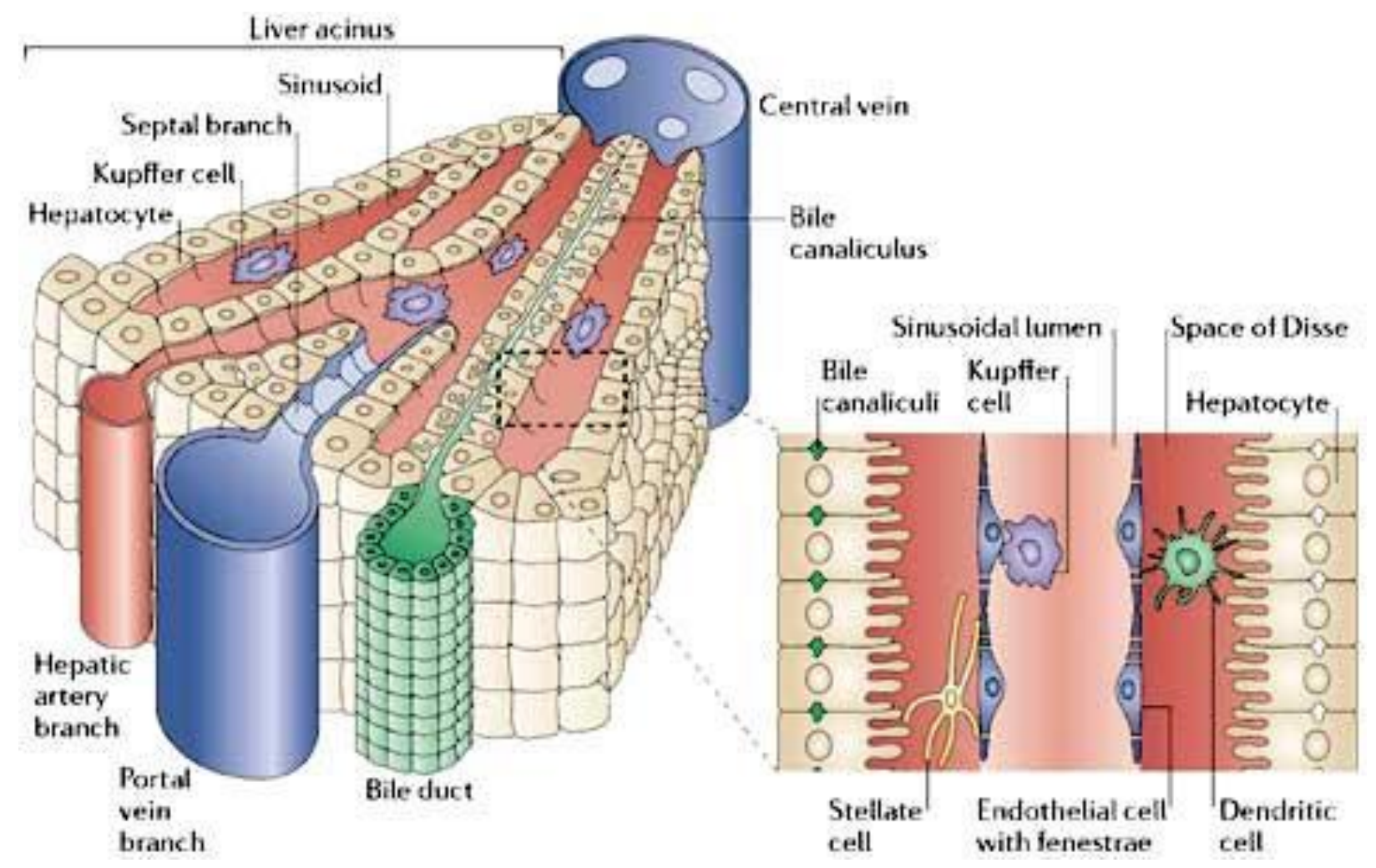

Figure 2. Hepatic cells organisation in the human liver lobules. Image from [14].

\subsubsection{Liver Extra Cellular Matrix (ECM)}

In contrast to other epithelial organs, the liver sinusoids lack a basement membrane that separates the epithelial cells (hepatocytes) from endothelial cells (LSECs). The sinusoids contain only a moderate ECM deposition primarily composed of fibronectin, collagen type I, and minor amounts of collagen types III, IV, V, and VI [7].

The liver ECM plays a key role in maintaining the healthy phenotype of the hepatic cells, thus contribute to the mechanical properties of the liver [15], provide a surface for cell adhesion, cell growth and migration, acts as reservoir for signalling molecules [16], and promote the differentiation and the expression of liver-specific functions and cell differentiation [17].

Hepatic cells differentiation, migration, proliferation, cell-cell interactions and cellmatrix adhesion is deeply correlated to the ECM composition, and its progressive qualitative and quantitative modifications, occurring during chronic fibrogenesis, 
dramatically affect the cellular behaviour, promoting the progression towards liver cirrhosis and cancer [18] [19].
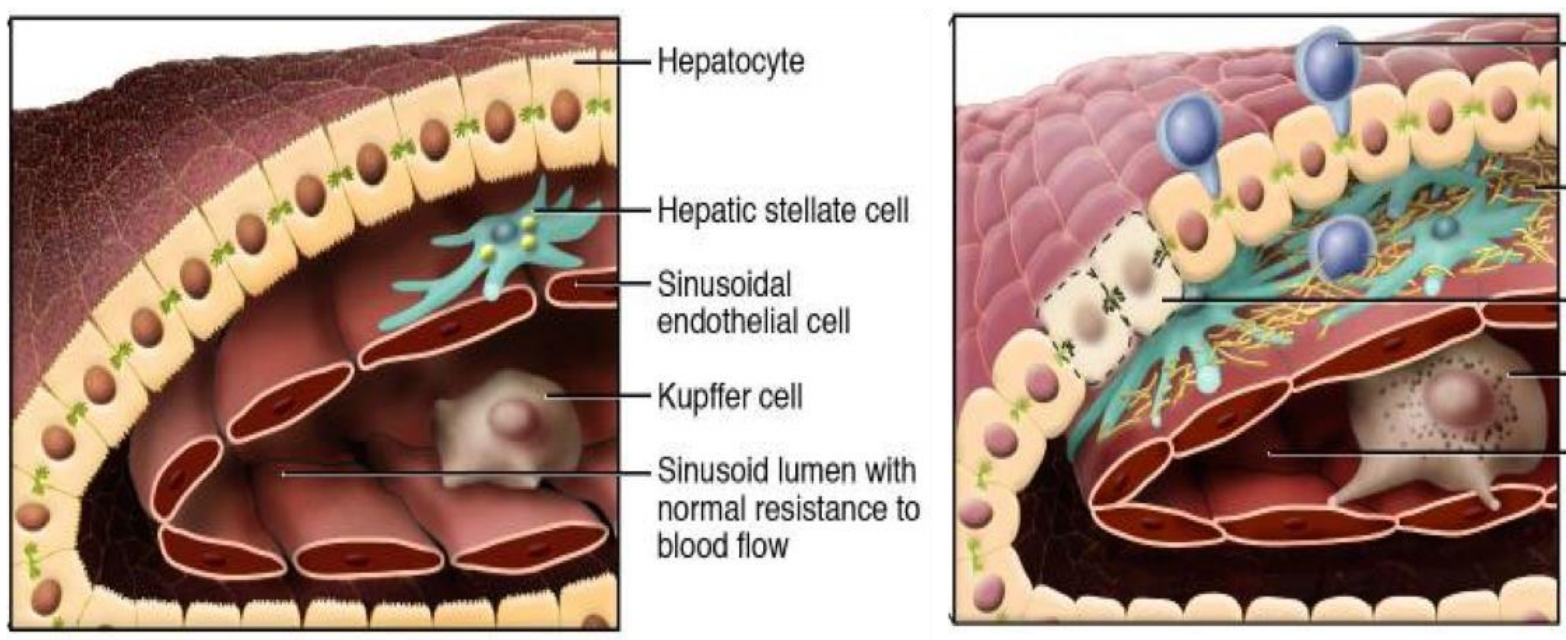

Figure 3. Representation of liver sinusoid in health liver (left Figure) and in diseased liver (right Figure) where activated hepatic stellate cells (HSC) increased the deposition of extracellular matrix (ECM) components in the space of Disse. This promotes changes in the cellular behaviour of other hepatic cells and leads to fibrosis, cirrhosis and cancer. Image modified from Friedman et al 2004 [13].

\subsection{Acute liver failure}

Acute liver failure is considered a rare disease characterised by an incidence of approximately 10 cases per million every year that usually occurs in adults between 30 and 40 years old not affected by pre-existing liver disorders [20]. The clinical manifestation of acute liver failure includes hepatic dysfunctions, abnormal liver coagulopathy and in $50 \%$ of the cases leads to multi organ failure and death [21]. In the last decade, especially in the developed world, the survival rate has considerably increased thanks to the improvements in emergency liver transplantation [22].

In the developing world, hepatitis virus type A, B and E infections are the most common cause of acute liver failure, with a survival rate below $50 \%$ [23-25], while in the developed world drug-induced liver injury (DILI) is the major cause of this pathology, accounting for approximately $50 \%$ of the cases in the United States [26]. Regarding the UK, acetaminophen-induced hepatotoxicity is the major cause of acute 
liver failure [27]. Moreover, a 2016 report from Office for National Statistics showed that approximately 200 people died in 2015 in the UK due to paracetamol poisoning [28].

\subsection{Metabolic liver disorders}

Metabolic liver disorders are usually caused by single enzyme deficiency causing dysfunctions in the synthesis or catabolism of proteins, carbohydrates or fats [29]. Metabolic liver disorders are rare diseases, but when considered all together they are responsible for $30 \%$ of the paediatric liver transplants [30].

Metabolic liver disorders include:

Alpha-1-antitrypsin (AAT) deficiency. AAT deficiency is a liver disease characterised by a hereditary single gene defect, that affects 1:3500 individuals and currently 180,000 people worldwide [31]. This deficiency in adult patients causes a chronic obstructive airway disease due to lack of circulating protease inhibitor alpha1-antitrypsin produced by hepatocytes. In paediatric populations, this condition leads to neonatal cholestasis, chronic liver disease and liver failure [32]. Liver transplantation is the only effective treatment to cure alpha-1-antitrypsin deficiency [33].

Wilson's disease. Wilson's disease affects one in 30.000-100.000 individuals and was first described by Wilson in 1912. It is a genetic disorder of copper metabolism characterised by severe movement disorder and liver syndromes including chronic hepatitis, cirrhosis and acute liver failure. The gene responsible for Wilson's disease is the ATPase copper transporting beta (ATP7B) gene, located on chromosome 13 and highly expressed in the liver, kidney, and placenta. ATP7B encodes for an ATPdependent copper transporter (ATP7B) [34], and defects in this enzyme cause accumulation of copper initially in the liver and later in other organs including the brain and kidney, causing liver and neurological manifestations [29]. 
Urea cycle disorders. The Urea cycle is characterised by consecutive biochemical reactions that allow the detoxification and conversion of ammonia into urea, a nontoxic excretory molecule [35]. These reactions are performed only by hepatocytes, and deficiency in one of the enzymes cause an increase of ammonia levels in the blood, which is highly neurotoxic [36]. The urea cycle disorders include Carbamyl Phosphate Synthetase (CPS) deficiency and Ornithine Transcarbamylase (OTC) deficiency [36, 37].

Crigler-Najjar (CN) Syndrome. The CN syndrome is caused by mutations in the gene encoding for uridine diphosphate glucuronosyltransferase 1 family, polypeptide A1 (UGT1A1) [38]. Defects in the activity of this enzyme cause hyperbilirubinemia, and when untreated can lead to kernicterus [29]. There are two different phenotypes of this disease: $\mathrm{CN} 1$ and $\mathrm{CN} 2$. The CN2 phenotype can be treated with phenobarbital, which increases the enzyme activity and allows to control jaundice. On the contrary, CN1 does not respond to phenobarbital induction and at the moment liver transplantation is the only definitive treatment [39].

Tyrosinemia Type I (TTI). TTI is a disorder caused by a deficiency in fumaryl acetoacetate hydrolase, the last enzyme involved in the tyrosine catabolism pathway. This enzymatic defect causes an accumulation in the hepatocytes and renal tubular epithelial cells of highly toxic metabolites, including fumarylacetoacetate and malelylacetoacetate [40], that trigger cell apoptosis and lead to acute liver failure, chronic liver disease, hepatocellular carcinoma (HCC), renal tubular dysfunction, and neurological complications [41]. Liver transplantation is usually required when acute liver failure occurs or when medical treatment fails [42].

Primary Hyperoxaluria. Primary hyperoxaluria is caused by a deficiency of the peroxisomal enzyme alanine-glyoxylate amino-transferase (AGT) [43] that lead to an excessive oxalate production. This enzymatic defect causes renal damage due to the accumulation and formation of calcium oxalate stones in the renal tubules and urinary tract. AGT is expressed in hepatocytes, and therefore liver transplantation acts as enzyme replacement therapy [44].

Glycogen Storage Disease (GSD). These liver diseases are caused by hereditary defects of different enzymes expressed in the liver, or in muscles, which are involved 
in the synthesis or catabolism pathways of glycogen. More than twelve types of glycogen storage disorders are known with an estimated incidence of 1 case every 20.000-40.000 individuals [45]. Hepatic GSDs include types I, III, IV, VI, and IX. The last 2 conditions tend to be mild and generally do not require liver transplantation [29].

Disorders of Bile Acid Synthesis. This group of liver disorders include nine different hereditary deficiencies of single enzymes involved in bile acid synthesis pathway [46]. All together, these metabolic liver disorders are responsible for $2 \%$ of persistent cholestasis in infants and if not treated, cause severe liver dysfunctions and may require liver transplantation [47].

Hemochromatosis. Hemochromatosis is a metabolic disorder that affects iron absorption, resulting in the accumulation of excess iron in different organs, in particular liver, heart and pancreas [48]. Hemochromatosis can be either genetic or acquired. Hereditary hemochromatosis $(\mathrm{HH})$ is the more common type and accounts for more than $80 \%$ of the cases [49]. Mutations of the gene HFE, which encodes a non-classical HLA class I protein involved in iron absorption, are the most common cause of hereditary hemochromatosis. The two homozygous mutation of the HFE gene C282Y and H63D account for the majority (85\%) of cases [50]. Left untreated, $\mathrm{HH}$ may lead to the life-threatening development of liver fibrosis, cirrhosis, hepatocellular carcinoma, cardiomyopathy, diabetes and other diseases such as arthritis and skin hyperpigmentation [51].

\subsection{The treatment of end-stage liver diseases: need for new treatment strategies}

In the United Kingdom, the incidence rate of liver disease is dramatically increased since 1970 and it is considered the fifth most common cause of death [52].

At the moment, liver transplantation is the only effective treatment for advanced liver diseases [53] but is limited by high costs and deficiency in donor organs and $15 \%$ of patients die while are waiting for liver transplantation [54]. 
Thus, an urgent need of new treatments is required as valid alternative to orthotopic liver transplantation.

\subsubsection{Alternative strategies for the treatment of end-stage liver diseases}

In the last decades, several strategies, including the use of extracorporeal bio-artificial liver devices [55, 56] and cell therapy [57, 58], have been tested as alternative approaches to liver transplantation for the treatment of acute liver failure or metabolic liver dysfunctions [55].

Bio-artificial liver devices (BAL) are external apparatus used to temporarily replace the hepatic functions in order to allow the repair and regeneration of the patient's liver, or act as a bridge to transplantation [59]. Several studies demonstrated that artificial liver devices based only on detoxification functions, including single pass albumin dialysis (SPAD) or molecular adsorbent recirculating system (MARS), were not able to significantly enhance survival in clinical trials $[60,61]$. More promising is the development bio-artificial liver devices functionalised with liver cells, since they are able to replace not only detoxification functions but also other important hepatic functions, including biotransformation, excretions and synthesis functions [61]. Selden et al. developed a bio-artificial liver device composed of alginate beads functionalised with HepG2 cells [62]. In this external device, alginate encapsulation imposes threedimensional growth of HepG2 with consequent high cell-to-cell contact and formation of HepG2 spheroids, resulting in increased cell functions up to ten-fold compared to conventional 2D monolayer culture systems [63]. Resulting BAL, when tried in a pig animal model, showed to improve several aspects of acute liver failure, such as acidosis, conjugation of bilirubin, and ammonia levels [64].

Cell therapy: hepatocyte transplantation. This approach was tested as an alternative to liver transplantation in order to replace the enzymatic defects in patients with metabolic liver disorders or to boost liver functions in patients diagnosed with acute liver failure [65]. Unfortunately, the successful rate of this therapy is limited due to poor hepatocyte survival and engraftment and to date this alternative treatment has not been able to reduce the need for organ transplantation [66]. Another important drawback is the limited source of available organs for the isolation of hepatocytes, 
considering that the main source for cell isolation derives from livers unsuitable for transplantation [67], i.e. ischaemic and/or abundantly steatotic. Recently, to overcome this problem and to increase the pool of available organs, the livers from non-heart beating donors have been tested for hepatocytes isolation, although cell viability and functionality of isolated hepatocytes was reduced compared to cells isolated from conventional ischaemic and/or abundantly steatotic livers [68].

\subsection{Implantable tissues for liver therapies}

As previously mentioned, the poor cell engraftment and survival are the main limitations correlated to the low successful rate of cell therapy for the treatment of liver disease. A promising strategy to overcome these problems is the development of in vitro scaffolds bioengineered with hepatocytes prior the in vivo implantation [69]. To improve the clinical treatment of acute liver failure or metabolic liver disorders, bioengineered implantable liver tissues should guarantee long-term engraftment and survival of hepatocytes as well as maintenance of their unique metabolic and synthetic functions after implantation [65].

Several natural and synthetic biomaterials characterised by adequate physiological, biochemical and biocompatible properties [70, 71], as well as different production methodologies, including cell encapsulation, 3D bio-printing and decellularizationrecellularization techniques, have been tested for the realization of functional implantable hepatic tissues.

Cell Encapsulation. Following this technique, a semipermeable polymer is employed to embed hepatic cells [72] in order to reduce the exposure of the implanted cells to host immune cells allowing at the same time the diffusion of oxygen and nutrients to maintain the cells alive [73]. Rat hepatocyte encapsulated in alginate beads and transplanted intraperitoneal in a rat animal model of acute liver failure improved liver functions up to 7 days [74]. The major drawbacks of this promising technique included the risk of an inflammatory reaction caused by the biomaterial [75] and the use of toxic 
cross-linking agents for the preparation of the implantable hepatic tissue that significantly reduced cell viability [76].

3D Printing. Using this novel approach, hepatic cells are combined with biocompatible materials, called bio-inks, to produce 3D functional constructs able to support cell viability and functionality [77]. Several natural and synthetic biomaterials, including decellularized extracellular matrix derived from different organs and tissues, have been employed as bio-inks for 3D printing $[78,79]$. In this context, porcine liver ECM bio-ink showed to improve the differentiation and metabolic activity of stem cells compared to monolayers of cells cultured on collagen type I [80].

Synthetic and natural scaffolds. Other approaches for the development of functional 3D scaffolds for in vitro culture and in vivo applications, consists on seeding the cells on 3D scaffolds composed of synthetic or natural biomaterials. Advantages of using synthetic scaffolds compared to natural scaffolds include easy manufacturing process, high reproducibility and good mechanical properties, but they lack some key properties, in particular bioactivity and biocompatibility [81]. The most common biomaterials used for the preparation of 3D scaffolds for biomedical applications include synthetic polylactide-co-glycolide, polyethylene glycol, and polycaprolactone (PLC) [80], or natural agarose, alginates and celluloses [82]. Furthermore, 3D scaffolds can be prepared using ECM-derived materials including type I collagen, elastin, laminin, fibronectin or hyaluronic acid. These ECM components are characterised by high biocompatibilityand bioactivity properties [83]. Nevertheless, several studies demonstrated that scaffolds composed of one or combinations of ECM components are not able to fully recapitulate the natural liver ECM microenvironment leading to a reduced hepatocyte viability and functionality [84].

Decellularization-recellularization. Decellularization process consists in the complete removal of cellular material from the tissue or organ while preserving ECM composition and $3 \mathrm{D}$ architecture of the native tissue $[85,86]$. This technique allows to overcome the previous described limitations and to develop functional scaffolds, able to mimic the natural microenvironment of tissues and organs. The decellularization followed by cell reseeding became an attractive technique for tissue and organ regeneration [87]. 
A promising methodology for cell delivery is the development of hydrogels composed of tissue-specific ECM obtained through the decellularization technique. Decellularized rat liver was used to develop hydrogels for both in vitro culture and in vivo transplantation of rat hepatocytes. Authors showed that in vitro culture of rat hepatocytes on ECM-hydrogels exhibited higher cell viability and functionality compared to collagen type I-coated plastic. Furthermore, rat ECM hydrogels containing rat hepatocytes were able to maintain the cells alive and functional after transplantation [88]. Porcine liver ECM hydrogels have also been used for hepatocyte transplantation, resulting in a better hepatocytes engraftment, viability and functionality compared to the classical infusion of hepatocytes [89].

To improve metabolic liver functions and increase the successful rate linked to cell therapy, an implantable liver tissue should replace at least $5 \%$ of the hepatic mass, while a larger hepatic tissue, at least $30 \%$ of the liver mass, is needed to fully replace a human liver [90]. Decellularization-recellularization of partial (i.e. left lobe) or whole liver represent an ambitious strategy to increase the pool of available organs for transplantation [91]. One of the biggest limitations of this approach is the number of hepatic cells needed. Indeed, a liver function less than $30 \%$ is not compatible with life [64] andaround 30 billion are required 80 billion hepatocytes are needed to obtain a transplantable functional liver suitable for a person of $70 \mathrm{Kg}$ average weight [92]. 


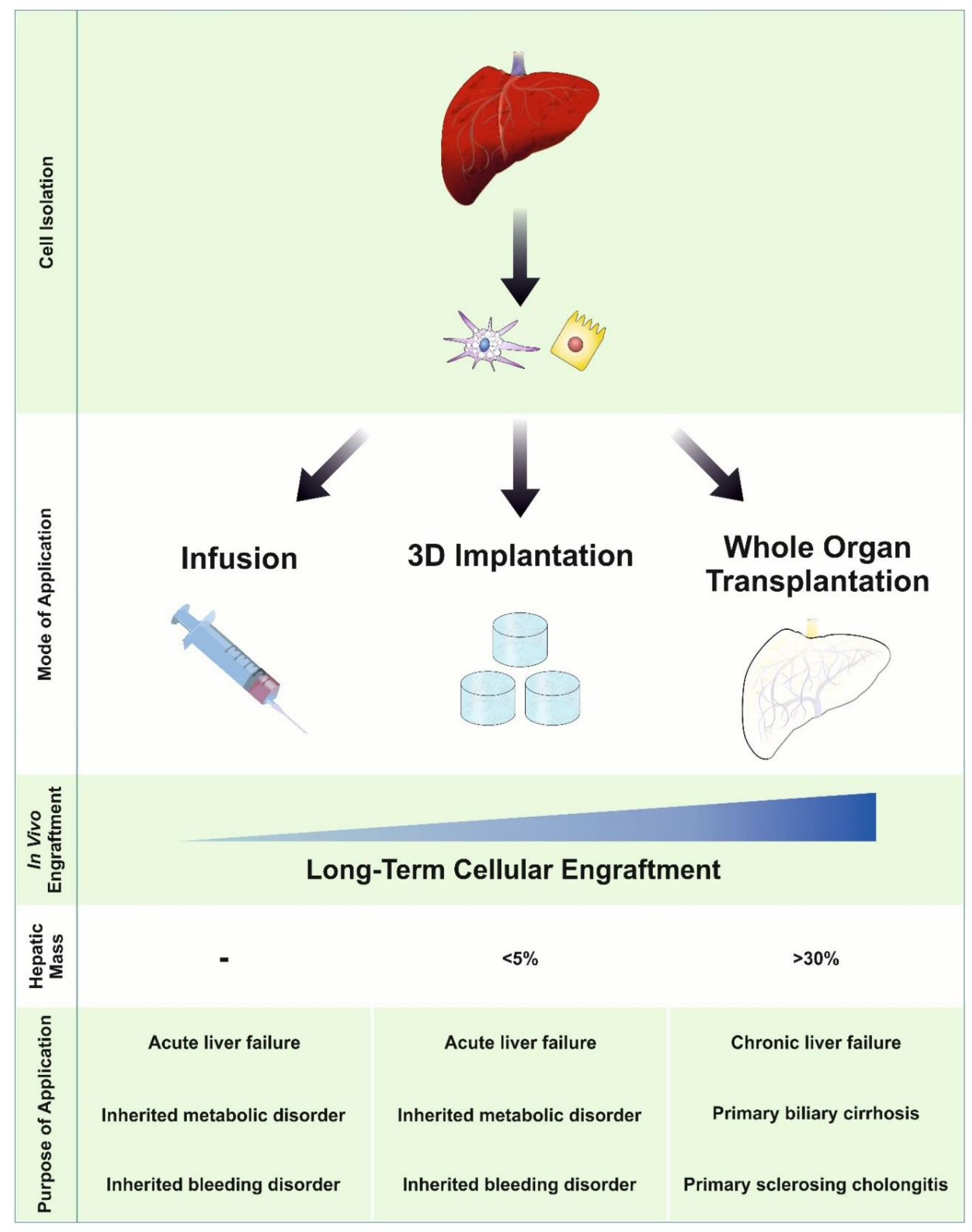

Figure 4. Tissue engineering strategies for the treatment of liver disease. The infusion of hepatocytes is already used in clinic for the treatment of metabolic liver disorders. A valid approach to improve the long-term engraftment and survival of hepatocytes after transplantation, is the bioengineering of implantable hepatic tissues able to replace $5 \%$ of the liver mass. Development of bioengineered whole liver allows to achieve more than $30 \%$ of liver mass and functionality thus increasing the pool of available organs for transplantation. Image modified from Mazza et al. [65]. 


\subsection{Current in vitro and in vivo liver disease models}

Liver disease models used to study liver disease and to develop/screen new drugs can be classified in two main groups: in vitro models and in vivo or animal models. Unfortunately, none of these models are able to fully recapitulate the complexity of human liver pathophysiology as confirmed by the high failure rate of drug candidates during clinical trials [93] and this due to lack of efficacy or safety issues when tested in humans [94-96].

\subsubsection{In vitro models}

To date, the majority of in vitro experiments performed in the drug discovery and drug development process are performed using 2D monolayers of cells grown on rigid plastic surfaces [97, 98]. In vitro culture of primary human hepatocytes using conventional two-dimensional (2D) culture systems is characterised by low hepatocytes viability and rapid dedifferentiation with consequent loss of their specific function such as ammonia metabolism, hepatic transport activity, albumin secretion and polarity [99]. Several attempts have been made to overcome these limitations and perform long-term in vitro studies. This include the pre-coating of plastic surfaces with different ECM proteins such as collagens, proteoglycans, laminin, fibronectin, but none of these 2D-ECM coated systems were able to avoid dedifferentiation of hepatocytes [99]. Better results in terms of hepatocytes viability and functionality were obtained using a sandwich culture system, in which cells are cultured in between two layers of collagen type I [100]. However, the sandwich system is characterised by high variability and lack of 3D organization, therefore is not able to mimic the native liver microenvironment [101, 102].

As a consequence, available $2 \mathrm{D}$ in vitro liver models are not efficient predictive tools to quantify in vivo drug efficacy [103-105]. Indeed, most of drug candidates showing high efficacy in in vitro models do not replicate the same performance when tested in vivo [106]. 


\subsubsection{In vivo models}

In vivo models have been extensively used for the development and testing of new drugs for the treatment of liver disease [107] because they are able to overcome some limitations associated to the 2D in vitro models including 3D architecture, cellular heterogeneity and provide a more physiological hepatic microenvironment [108]. Nevertheless, available animal models are not able to replicate human liver diseases and the numerous interspecies differences in gene and protein expression compromise their effectiveness to study drug efficiency, pharmacokinetic, metabolism and safety [109, 110]. Furthermore, these animal models are expensive and timeconsuming [111].

This emphasises the need of new models able to mimic the human liver microenvironment for the study and validation of new targets as well as to better predict drug efficacy and safety, reducing at the same time the costs of drug discovery and development process. In the last decades, research moved in this direction to develop alternative in vitro 3D models that can replace conventional 2D plastic system for the high throughput screening (HTS) of new drugs and reduce the use of animal models [112].

\subsection{D vs. 3D ECM-based in vitro systems}

3D ECM-based in vitro models present important features of native tissue microenvironment, including retention of ECM components, 3D architecture and tissue-specific biochemical and mechanical cues. These models allow more physiological cell-cell interactions and cell-ECM interactions, with oxygen and nutrients gradients allowing the cells to mimic better their in vivo behaviour compared to the 2D cell culture platform $[113,114]$.

Therefore, 3D ECM-based in vitro models are suitable platforms to study the mechanisms underlying human liver diseases, validate new targets and investigate drug efficacy and toxicity. 


\subsubsection{Cell-cell interactions}

Cell-cell interactions regulate several cellular mechanisms including differentiation, migration and proliferation [115]. Cadherins are transmembrane proteins that play a key role in the adherens junctions formation and cross talk between neighbouring cells by transducing mechanical signals into signalling cascades that regulate the cellular behaviour [116]. This emphasises the importance of 3D in vitro models that enable to mimic the natural cell-cell adhesions and interactions and therefore preserving the in vivo like cellular behaviour [117].

Moreover, a 3D co-culture of multiple cells is essential to recapitulate the high level of organization and interactions between the different hepatic cell types that characterize the liver microenvironment [118]. Indeed, several studies showed a better viability and preservation of hepatocytes specific functions when co-cultured with hepatic stellate cells $[119,120]$.

\subsubsection{Cell-ECM interactions}

The interactions between hepatic cells and ECM components lead to bidirectional chemical and mechanical stimuli that regulate the cellular phenotype and functions and as a consequence this may affect the ECM composition [121, 122]. Integrins are transmembrane proteins that mediate the interaction between cells and ECM components regulating cell survival, motility, migration, phenotype and functionality [123]. This highlight the importance of using 3D models composed of tissue-specific ECM to mimic the in vivo cell behaviour, functionality and phenotype [122]. In this context, several studies showed significant differences in cell phenotype and functionality when cells are cultured in 2D plastic systems versus 3D ECM-based models, further leading to different cell responses after drug treatment [121, 124]. 


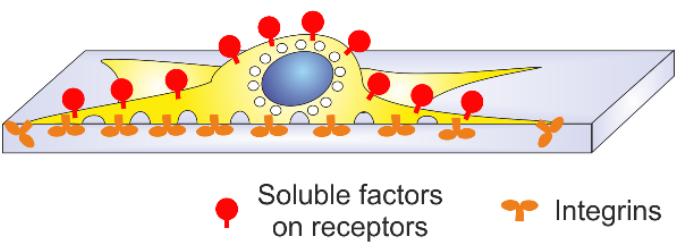

Cells on a Traditional 2D Surface

Adopt an unnatural flat and elongated shape

Large proportion of cell covered by plastic

Absence of exogenous signalling factor gradients

Stiffness of material is $>2 \mathrm{GPa}$

Lack of ECM-specific bioactive cues

Cell-cell interactions only on $x-y$ plane

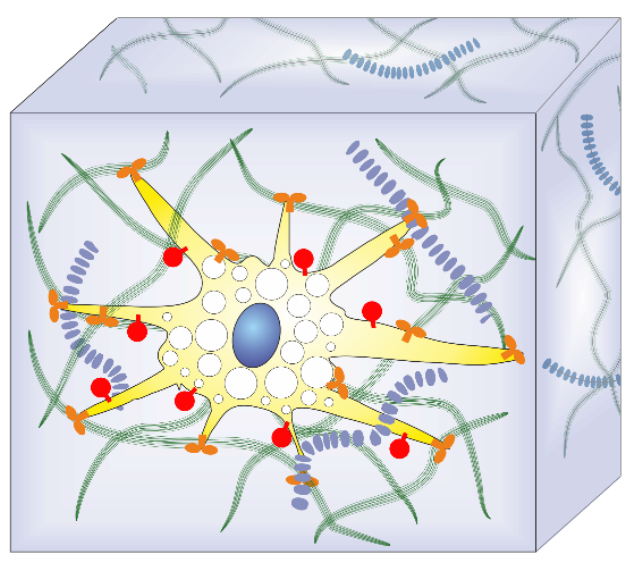

Matrix proteins 'monomon Proteoglycans

Cells maintain natural morphology

Large proportion of cell in contact with surrounding microenvironment

Presence of exogenous signalling factor gradients

Stiffness of material in the region of $1-20 \mathrm{KPa}$

Presence of ECM-specific bioactive cues

Cell-cell interactions on all planes

Figure 5. Schematic cartoon comparing the effect of 2D (a) and 3D (b) cultures on cells. 3D culture microenvironments maintain essential cellular cues. Image modified from Mazza et al [125].

\subsection{D in vitro hepatic models}

During the last decade, different methodologies have been investigated for the development of functional 3D in vitro hepatic models able to mimic the natural and diseased liver microenvironment [126]. This include the development of cell spheroids, cell sheet stacking, cut liver slices and scaffolds models.

\subsubsection{Spheroids}

This is one of the first developed 3D cell culture system and is based on the selforganisation of hepatic cells into 3D spherical multicellular structures within a soft biomaterial [127]. When hepatocytes are organised in spheroids cells produce ECM, 
therefore this technology allows both cell-cell interactions and cell-ECM interactions $[128,129]$. For these reasons the spheroids 3D culture enables a better preservation of hepatocytes-specific functions compared to conventional 2D plastic cell cultures [130]. Moreover, spheroids demonstrated to better predict the in vivo drug efficacy and toxicity compared to $2 \mathrm{D}$ plastic systems $[131,132]$.The main limitation of this 3D cell culture technology is the difficulty to maintain a uniform size of the spheroids that compromises the reproducibility of the experiments [133].

\subsubsection{Cell sheet stacking}

This technology is based on the use of thermo-responsive polymers that allow the development of cell sheets that are stacked on top of each other to create a 3D model [134]. Poly (N-isoproplyacrylamide) (PIPAAm) is one of the most used polymers in cell sheet technology because it allows the easy preparation of cell sheets and cell retrieval by changing the working temperature [135]. Indeed, below $20^{\circ} \mathrm{C}$ the polymer is hydrophilic and cells are not able to attach to the surface of the material while when the temperature increases up to $37^{\circ} \mathrm{C}$ the polymer becomes hydrophobic allowing cell engraftment. Different studies showed the development of hepatic cell sheets characterised by higher hepatic functions compared to standard 2D cell culture systems $[126,136]$.

The major drawback of this model is limited diffusion of nutrient and glucose that allow culturing the cells only for a short period $[137,138]$.

\subsubsection{Precision Cut Liver Slices}

These 3D models are developed by sectioning the liver into thin layers of approximately $250 \mu \mathrm{m}$ that are used as in vitro culture models. Liver slices can be prepared from livers obtained from different species including rat [139], mouse [140] and human [141] by retrieving cylindrical core biopsies that are further cut into thin slices using tissue slicers. There are different incubation systems used to maintain the hepatic cells alive during the in vitro culture. The most widely applied incubation 
systems are using 6- or 12-well culture plates [142]; with the dynamic organ culture system mostly used for long-term culture(>72 hours) [143], and the rocker incubator, in which slices are cultured on a Netwell insert in six-well plates [144]. In this 3D model, most researchers use culture media such as Williams medium E, supplemented with glucose and antibiotics and saturated with $95 \% \mathrm{O}_{2} / 5 \% \mathrm{CO}_{2}$. For long-term culture (>48 hours), other supplements including insulin, glucagon, corticosterone, epidermal growth factor and/or foetal calf serum are needed to improve cell viability [145]. The major advantages of this 3D model are the preservation of the natural liver microenvironment in terms of 3D architecture, cell heterogeneity and cell functionality [146]. On the contrary, the major limitation of using liver slices is the short length of in vitro culture capability due to the fast reduction in hepatic cell viability and functionality over a period of 1 to 7 days [147].

\subsubsection{Scaffold based 3D models}

3D in vitro culture systems can also be developed by reseeding cells into scaffolds derived from both natural and synthetic sources. Most common synthetic materials used for the development of liver 3D models includes Poly-ethylene glycol (PEG) [148], Poly-lactic acid (PLA) [149] and Poly-caprolactone (PCL) [150]. Even more, natural polymers can be employed for the development of these scaffolds. The most common natural sources are chitosan [151], cellulose [152] or alginate [153]. These models are easy to prepare and to modify in terms of topography and mechanical properties, but the lack of tissue specificity and not being able to replicate the microenvironment of the organ under investigation are limitations. Decellularized liver scaffolds, obtained through decellularization-recellularization technique present several advantages compared to the previously mentioned natural and synthetic scaffolds, including preservation of native liver 3D architecture and ECM composition, allowing to fully reproduce the liver microenvironment. Human primary hepatocytes and HepG2 cells cultured in this 3D hepatic model maintained their specific metabolic functions during the in vitro culture [154]. Limitations of this model include the time needed to prepare the scaffolds, the high number of cells needed to develop the 3D hepatic models and the co-culture limitations due to variability in cell engraftment. 
These limitations preclude the use of decellularized 3D liver scaffolds for high throughput screening of new drugs for the treatment of liver diseases. 


\section{Chapter 2: Development of human liver ECM-hydrogels for liver bioengineering and cell transplantation}

\subsection{Introduction}

This Chapter describes the development of human liver ECM-hydrogels through the optimisation of a novel protocol for the solubilisation of human liver ECM powder without the implementation of xenogeneic enzymes for the digestion of the liver ECM components. Experiments described in this Chapter will also show the feasibility of using ECM-hydrogels in in vitro and in vivo applications.

\subsubsection{Hydrogels for tissue engineering and regenerative medicine}

Tissue engineering is a key area of regenerative medicine with the aim of producing functional cells, tissues or organs in order to replace damaged tissues/organs to restore their normal functions and/or allow tissue regeneration [155, 156]. Another important aim in this field is to create in vitro models to study the mechanisms of human diseases and develop safe and effective treatments for patients [157].

In the last decades hydrogels have been increasingly used for tissue engineering and regenerative medicine applications due to their excellent biocompatibility, biodegradability, low toxicity, similarity of their mechanical properties to those of natural tissues, and simplicity of their preparation [158]. In this context, several studies demonstrated the feasibility of using hydrogels for regenerative applications of different organs and tissues, including bone [159, 160], cartilage [161, 162], muscle [163], blood vessels [164, 165] heart [166, 167], kidney [168], liver [169, 170] and brain [171]. 


\subsubsection{Natural and synthetic hydrogels}

Hydrogels, according to the biomaterial used for their preparation, can be classified in two main classes: synthetic and natural.

Synthetic hydrogels are commonly characterised by strong mechanical properties in terms of stiffness and viscosity, long term stability, high reproducibility, and for these reasons many synthetic biomaterials are FDA-approved [170]. Most common synthetic hydrogels include Poly-ethylene glycol (PEG) [148], Poly-lactic acid (PLA) [149] and Poly-caprolactone (PCL) [150]. On the contrary, natural hydrogels are characterised by poor mechanical properties, high biodegradability, fast biodegradation, low cytotoxicity and higher bioactive properties when compared to synthetic hydrogels [172]. Most used natural hydrogels for tissue engineering and regenerative medicine applications include chitosan [173], cellulose [152] and alginate [153]. Both synthetic and natural hydrogels lack tissue specificity, the characteristic architecture and the microenvironment of the organ under investigation. In addition, hydrogels can be developed by using biological materials ECM-construct derived. For instance, different hydrogels have been developed from components of the extracellular matrix (ECM) such as collagen, hyaluronic acid, laminin, fibronectin and elastin [174]. These hydrogels are characterized by preservation of ECM molecules that improve cell attachment, proliferation and differentiation. However, several studies demonstrated that individual ECM components, or combinations of, were not able to prevent dedifferentiation of hepatocytes [175-177].

Matrigel is a thermoresponsive hydrogel composed of proteins secreted by Engelbreth-Holm-Swarm sarcoma cells [178], which primarily consists of laminin, collagen IV and entactin [179]. Matrigel was found to be superior to collagen and other above-mentioned ECM based scaffolds. Indeed, cultured rat hepatocytes exhibited better preserved liver-specific functions [180]. However, due to its murine and tumorigenic source, Matrigel was shown to contain more than 100 mouse growth factors including transforming growth factor beta (TGF- $\beta$ ), epidermal growth factor (EGF), cytokines and other proteins involved in the inflammation/tissue remodelling response [181]. For these reasons, Matrigel is not the ideal biomaterial to use for clinical applications. Another limitation of using Matrigel is the high batch-to-batch variability that can compromise the reproducibility of the experimental results [182]. 
Many decellularised tissues obtained from different organs and species have been utilized to develop ECM based hydrogels [183]. Examples include porcine small intestinal submucosa [184], rat liver [185], porcine liver [175, 186], porcine urinary bladder [113] and porcine adipose tissue [187]. These studies showed a clear benefit in using ECM based hydrogels for in vivo and in vitro applications. However, the physical and biochemical composition of the ECM is tissue-specific [188], therefore these differences compared to human liver can affect the reproduction and maintenance of specific functions of human primary hepatocytes [189]. From the physiological and anatomical point of view, porcine liver is considered to be the most similar to human liver compared to rat and mouse [190]. As shown in Figure 2.1, porcine liver (a) is characterised by well-defined interlobular septa from connective tissue that are absent in a healthy human liver (b). In contrast, in a fibrotic human liver, the increased deposition of fibrillar ECM components, leads to the formation of interlobular septa similar to that of porcine liver. Therefore, although porcine liver ECM is considered to be the closest to human liver ECM, these changes in the ECM composition might alter cell signalling leading to a non in vivo like behaviour of hepatic cells [191].
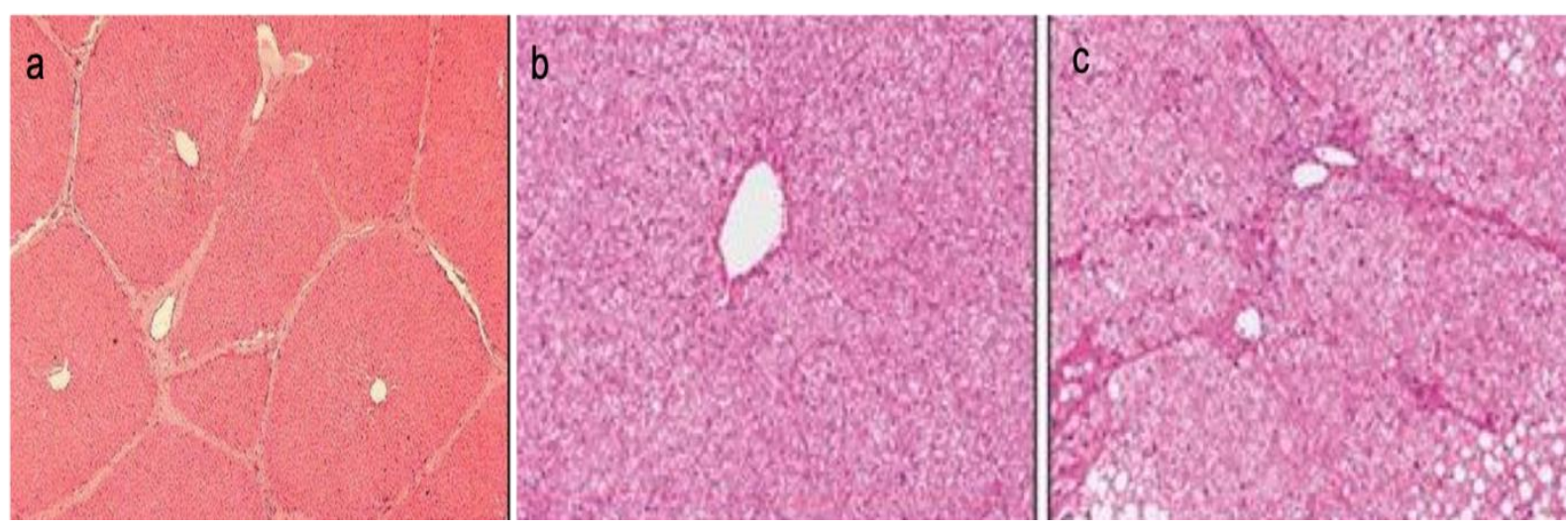

Figure 6. Haematoxylin and Eosin staining of (a) porcine liver and (b) human healthy liver. (c) Trichrome stain of a fibrotic human liver biopsy. Image (a) from [192], images (b,c) from Wanf et al [193].

\subsubsection{Hydrogel preparation methods}


Hydrogels can be prepared through different cross-linking methods: physical crosslinking, chemical cross-linking, enzyme cross-linking and ionic cross-linking [194].

\subsubsection{Physical cross-linking}

Most common physical cross-linking mechanisms for the preparation of hydrogels are triggered by changes in $\mathrm{pH}$ or temperature. The main advantages of employing these cross-linking methods are based on the simplicity of the process and that they do not require other cross-linking agents that might enhance the toxicity of the material [195].

Temperature-responsive hydrogels. Poly $(\mathrm{N}$-isopropylacrylamide) is one of the most used temperature-responsive biomaterial characterised by a sol-gel transition at approximately $32^{\circ} \mathrm{C}$ [196]. The main drawback of this synthetic polymer is based on its shrinking behaviour after gelation that affects the cell encapsulation process [197]. Furthermore, Poly(N-isopropylacrylamide) is not biodegradable and to overcome this problem different biodegradable polymers, such as chitosan [198] or dextran [199] have been combined with Poly( $\mathrm{N}$-isopropylacrylamide) to obtain biodegradable hydrogels for regenerative medicine applications.

Agarose is another temperature-responsive biomaterial whose cross-linking mechanism is characterised by the aggregation of double helical structures while the temperature decreases from melting point $\left(\sim 85^{\circ} \mathrm{C}\right)$ to the gel point $\left(\sim 35^{\circ} \mathrm{C}\right)$ [200].

pH-responsive hydrogels. Poly( $\mathrm{N}$-isopropylacrylamide) can be modified to respond to changes in the $\mathrm{pH}$ by combining it with $\mathrm{pH}$-sensitive polymers, including 2(diethylaminoethyl) methyl methacrylate [201]. However, due to the lack of biocompatibility and gel stability, $\mathrm{pH}$-responsive hydrogels are not considered the best choice for clinical applications [158].

\subsubsection{Chemical cross-linking}

Chemical cross-linked hydrogels are developed through the formation of covalent bonds between polymers. Chemical cross-linked hydrogels are characterised by high resistance to mechanical stimuli and significant volume changes after gelation. 
Chemical cross-linking hydrogels can be prepared through photopolymerization mechanism, Michael-type addition reaction or Schiff base reaction [158].

Photocrosslinked hydrogels. Photopolymerization is the most common technique used to prepare chemical cross-linking hydrogels. During this process a photoinitiator is exposed to visible or UV light with consequent formation of free radicals that interact with acrylate or methacrylate groups, triggering the formation of covalent bonds [202]. Different studies have reported the development of photocrosslinked hydrogels, including poly(ethylene glycol)-diacrylate (PEGDA) and poly(ethylene glycol)dimethacrylate (PEGDMA) for biomedical applications [203]. Furthermore, natural biomaterials including chitosan, hyaluronic acid alginate have been combined with PEGDA or PEGDMA to develop hydrogels with photo-crosslinkable properties [204206].

Michael-type addition reaction hydrogels. The covalent bond in the Michael-type addiction reaction occurs between thiols and acrylates. Acrylates are salts or esters of acrylic acid or its derivates. They are composed of vinyl groups directly attached to the carboxylic group. The advantage of using this method is the possibility of preparing chemical cross-linked hydrogels under physiological conditions, such as room temperature, neutral $\mathrm{pH}$ and using water as solvent for the reaction. Michael-type addition reaction was performed to develop hydrogels composed of thiol-modified hyaluronic acid and poly(ethylene glycol)-diacrylate that were able to support the in vitro culture of human stem cells and fibroblast [207].

Schiff base-crosslinked hydrogels. A Schiff base reaction allows the formation of a double bond between an amino-group and an aldehyde-group. Aldehyde groups introduced in modified polysaccharides, including hyaluronic acid or dextran, can form a double bond with the amino groups of other polymers, including $\mathrm{N}$-succinil-chitosan, to obtain a Schiff base-crosslinked hydrogels [208].

\subsubsection{Enzyme cross-linking}

The most common used enzymes to cross-link hydrogels for biomedical applications are transglutaminases and horseradish peroxidases. 
Transglutaminases are thiol enzymes that allow the formation of a covalent bond between the amine groups present in lysine amino acids and $\mathrm{y}$-carboxamide groups present in glutamine amino acids. Factor XIII is a transglutaminase enzyme that allows to develop hydrogels using fibrinogen and thrombin [209].

Horseradish peroxidase $(\boldsymbol{H R P})$ is a peroxidase enzyme that allows the formation of bonds between the phenol group of aromatic amino acids and aniline derivatives in the presence of peroxide [210].

\subsubsection{4 lonic cross-linking}

During ionic cross-linking, ionic polymers electrostatically interact with di- or tri-valent ions of opposite charge. lonic cross-linking is performed to develop alginate hydrogels in presence of calcium, a divalent cation that interact with anionic groups of alginate [211]. Chitosan-glycerol phosphate salt and chitosan-alginate hydrogels are other examples of hydrogels prepared through ionic cross-linking mechanism [212]. 


\subsection{AIM and OBJECTIVES}

\subsubsection{Aim}

To develop and characterize human liver extracellular matrix hydrogels that are able to provide a physiological microenvironment for in vitro culture and in vivo transplantation of human hepatic cells.

\subsubsection{Objectives}

To achieve this aim the following objectives will be considered:

1. Developing a suitable protocol for the solubilization and gelation of human liver ECM;

2. Evaluating the $3 \mathrm{D}$ ultrastructure and mechanical properties of ECM-hydrogels;

3. Assessing the in vitro biocompatibility by seeding human hepatoblastoma cells (HepG2) as a tool for Human Primary Hepatocytes;

4. Comparing gene expression and functionality of HepG2 cells cultured in ECMhydrogels and in acellular liver scaffolds. 


\subsection{METHODS and MATERIALS}

\subsubsection{Source of human livers}

The experiments described in this thesis were performed using human livers unsuitable for transplantation due to prolonged graft cold ischaemic time, the presence of extra-hepatic malignancy or other important extra-hepatic co-morbidities in donors or recipients. Livers used in this work were defined "healthy" since the histological analysis performed after the organs' retrieval did not prove evidence of any degree of tissue fibrosis and fat accumulation. The study was approved by the UCL Royal Free Biobank Ethical Review Committee (NRES Rec Reference: 11/WA/0077). Informed consent for research was approved by the NHSBT ODT organ retrieval pathway, as well as the NHSBT Research Governance Committee. Donor livers were processed in accordance with UCL Royal Free Biobank protocols under the Research Tissue Bank Human Tissue Act licence, prior to be used in research. Human livers obtained at the Royal Free London Foundation Trust were coordinated, received and recorded by the UCL Tissue Access for Patient Benefit organisation (TAPb) which links research activities between UCL Royal Free Biobank, the Royal Free Trust and UCL. TAPb has full governance in place for this purpose which involves the NHSBT ODT pathway, the Human Tissue Authority licencing, and the local Trust / UCL Research offices [154, 213].

\subsubsection{Tissue retrieval and preparation}

As mentioned above, healthy human livers, not suitable for transplantation, were retrieved, under local ethics, from the Royal Free BioBank. Upon retrieval, livers were perfused with 1\% Phosphate Buffered Saline (PBS; Sigma-Aldrich) to clear from blood, dried and stored at $-80^{\circ} \mathrm{C}$. 


\subsubsection{Development of Human Liver ECM-Hydrogels}

Human livers unsuitable for transplantation were decellularized following a novel protocol developed and published by Mazza et al. in 2015 [213]. The initial protocol (Protocol 1) tested for the development of human liver ECM-hydrogels, was aimed to compare the efficiency of previously published protocols for porcine liver [185, 186] with the decellularization of human livers. In the first step human decellularized liver was lyophilized (VIRTIS Benchtop) overnight to obtain a dried tissue that was then milled (rotary knife milling machine, Laval Lab) to create human liver ECM powder. $100 \mathrm{mg}$ of ECM powder was then digested with $10 \mathrm{mg}$ of pepsin (pepsin from porcine gastric mucosa, Sigma-Aldrich) in $10 \mathrm{ml}$ of $0.01 \mathrm{M}$ hydrochloric acid $(\mathrm{HCl}, \mathrm{VWR})$ at room temperature for 72 hours under magnetic stirring (Cole-Palmer). The $\mathrm{pH}$ of the resultant ECM solution was then neutralised with $0,1 \mathrm{~N}$ sodium hydroxide $(\mathrm{NaOH}$, Alfa Aesar) (1:10 volume of ECM solution) and the osmotic pressure was adjusted with sterile 10X phosphate buffer saline (PBS, Gibco) (1:9 volume of ECM solution). PBS solution 1X (Sigma-Aldrich) was added to achieve a final ECM concentration of 6 $\mathrm{mg} / \mathrm{ml}$. The last step was the incubation of the solution at $37^{\circ} \mathrm{C}$ for $1 \mathrm{~h}$ to gel the ECM solution.

Next, an alternative protocol was tested (Protocol 2) in which experimental steps were maintained identical to protocol 1, but the concentration of pepsin was doubled, and the final ECM concentration was increased up to $12 \mathrm{mg} / \mathrm{ml}$.

Lastly, a third protocol (Protocol 3) was investigated in which human liver ECM powder was suspended in $0.5 \mathrm{M}$ acetic acid (Thermo Fishes Scientific) (ECM concentration 4 $\mathrm{mg} / \mathrm{ml}$ ), without the implementation of pepsin, at $4{ }^{\circ} \mathrm{C}$ for 72 hours under magnetic stirring. Once suspended the resulting product has the appearance of a solution (i.e. it is clear). Human liver ECM solution was then ultra-centrifuged (Beckman Coulter, LE-80 K Ultracentrifuge) at $10000 \mathrm{~g}$ at $4{ }^{\circ} \mathrm{C}$ for 30 minutes. The pellet of human liver ECM was resuspended in acetic acid $0.25 \mathrm{M}$ (ECM concentration $6 \mathrm{mg} / \mathrm{ml}$ ) at $4{ }^{\circ} \mathrm{C}$ for 24 hours followed by sonication (Bandelin Sonoplus) (5 cycles of 20 seconds at 50 $\mathrm{Hz}$ ). The $\mathrm{pH}$ of the resultant ECM solution was then neutralised with $10 \mathrm{~N}$ sodium hydroxide (30 $\mu$ l per each $\mathrm{ml}$ of ECM solution) and the osmolarity was adjusted with 10X PBS (1:10 volume of ECM solution). Then 1X PBS was added to obtain the final 
ECM concentration of $4 \mathrm{mg} / \mathrm{ml}$. Again, during the last step the solution was incubated at $37^{\circ} \mathrm{C}$ for 1 hour to gel the ECM solution.

a

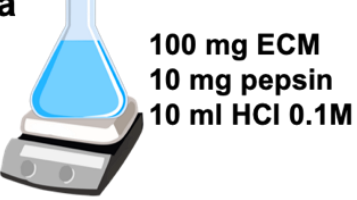

Solution is stirred for 3 days at room temperature b

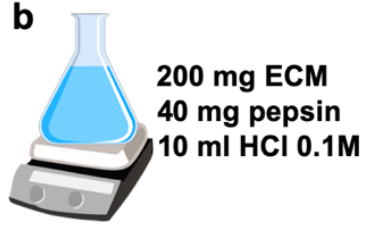

Solution is stirred for 3 days at room temperature
C

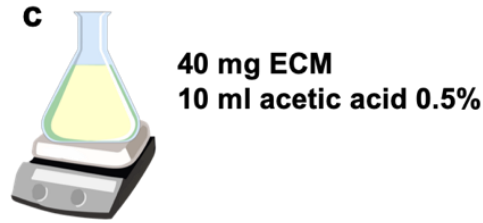

Solution is stirred for 3 days at $4^{\circ} \mathrm{C}$

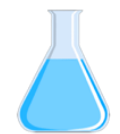

ECM concentration $10 \mathrm{mg} / \mathrm{ml}$

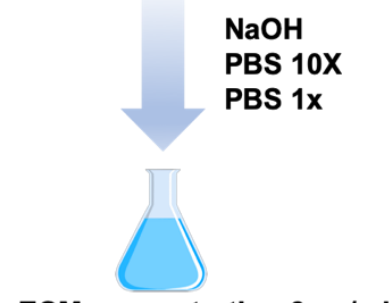

ECM concentration $6 \mathrm{mg} / \mathrm{ml}$

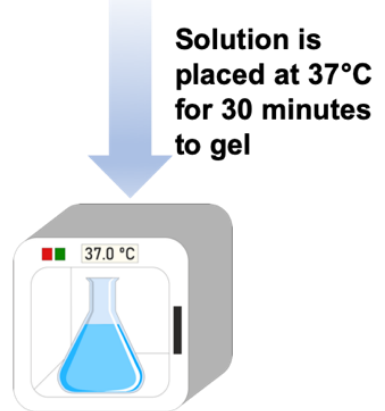

ECM concentration $20 \mathrm{mg} / \mathrm{m}$

$$
\begin{aligned}
& \text { NaOH } \\
& \text { PBS 10X } \\
& \text { PBS 1x }
\end{aligned}
$$

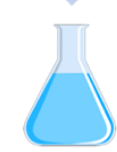

ECM concentration $12 \mathrm{mg} / \mathrm{ml}$

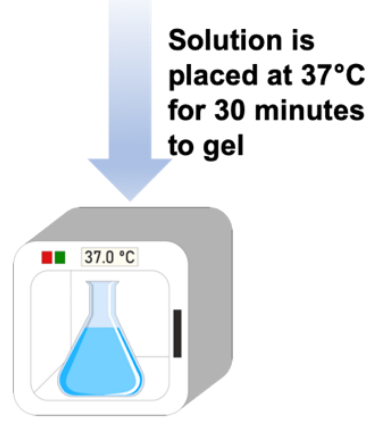

Centrifugation at $10000 \mathrm{~g}$ for 30 minutes

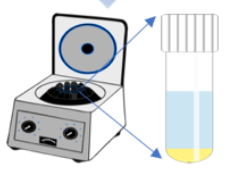

Pellet is resuspended in acetic acid $0.25 \%$ at $4^{\circ} \mathrm{C}$ for 1 day

Sonication

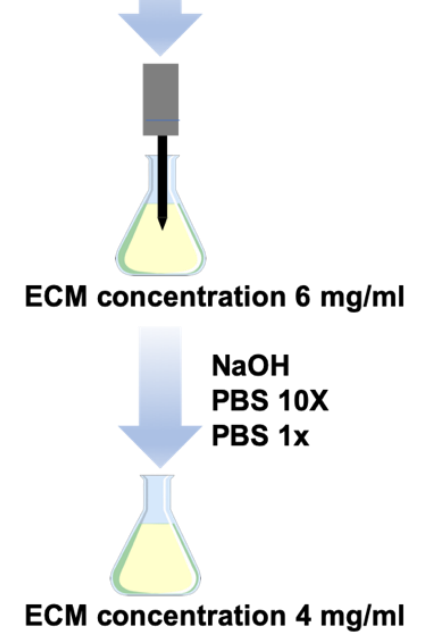

Solution is placed at $37^{\circ} \mathrm{C}$ for $\mathbf{3 0}$ minutes to gel

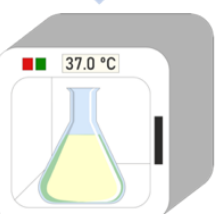

Figure 7. Schematic view of the protocols carried out to prepare the ECM liver solution. (a) Protocol 1 and (b) Protocol 2 were based on the use of hydrochloric acid and pepsin, while (c) Protocol 3 was based on the use of acetic acid without implementation of pepsin. 


\begin{tabular}{|c|c|c|c|}
\hline Protocol features & \multicolumn{1}{|c|}{ Protocol 1 } & Protocol 2 & Protocol 3 \\
\hline Acid used & Hydrochloric acid & Hydrochloric acid & Acetic acid \\
\hline $\begin{array}{c}\text { Xenogeneic } \\
\text { protease }\end{array}$ & $\begin{array}{c}\text { Porcine pepsin } \\
(10 \mathrm{mg} / 100 \mathrm{mg} \\
\text { ECM }) \text { in } 10 \mathrm{ml} \\
\mathrm{HCl} 0.01 \mathrm{~N}\end{array}$ & $\begin{array}{c}\text { Porcine pepsin }(20 \\
\mathrm{mg} / 100 \mathrm{mg} \text { ECM } \\
\text { in } 5 \mathrm{ml} \mathrm{HCl} 0.01 \mathrm{~N}\end{array}$ & Not used \\
\hline Sonication & Not performed & Not performed & $\begin{array}{r}5 \text { cycles of } 20 \\
\text { seconds at } 50 \mathrm{~Hz}\end{array}$ \\
\hline $\begin{array}{c}\text { Final ECM } \\
\text { concentration }\end{array}$ & $6 \mathrm{mg} / \mathrm{ml}$ & $12 \mathrm{mg} / \mathrm{ml}$ & $4 \mathrm{mg} / \mathrm{ml}$ \\
\hline
\end{tabular}

Table 1. Differences between the three protocols used to solubilise the ECM powder derived from human livers.

\subsubsection{Gelation of Human liver ECM solution}

The gelation of the human liver ECM solution was prepared following protocol 3 (acetic acid and sonication without the use of pepsin) and was obtained with the implementation of the gelling agent, Agarose (Sigma-Aldrich) or Agar (Sigma-Aldrich). Agar is a complex mixture of polysaccharides derived from red-purple seaweeds. The two major components of agar are agarose, the real gelling agent, and agaropectin that has a low gelling ability. These gelling agents are considered to be biocompatible, non-cancerogenic, non-toxic and completely biodegradable at low concentration [214]. Gels can be formed with Agar and Agarose in high diluted solutions, containing a fraction of $0.2 \%$ to $2.0 \%$ of the polymer. Agar and Agarose solutions form thermally hydrogels while being cooled down below their gelation temperature $\left(17-35^{\circ} \mathrm{C}\right)$.

Agarose or Agar powders were heated using a microwave (Sanyo. Super Showerwave) until complete solubilisation in 1X PBS was achieved and to obtain $2 \%$ w/v stock solutions. Resultant gelling agent solutions were cooled down and lastly, 
different volumes of agar and agarose solutions $(0.125-1 \% \mathrm{v} / \mathrm{v})$ were used to gel human liver ECM solution

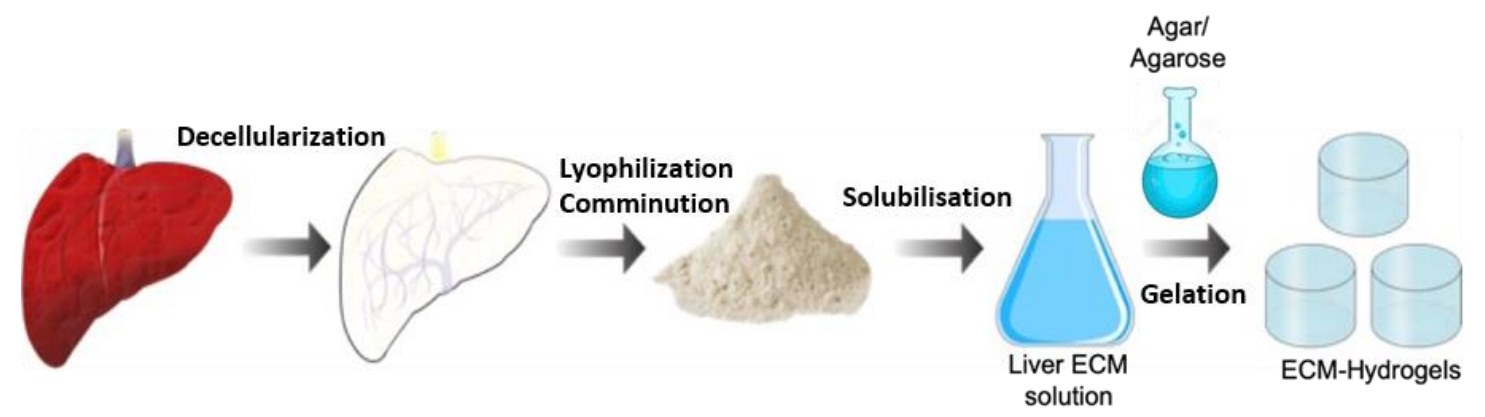

Figure 8.Schematic view of the process for producing hydrogels from human livers unsuitable for transplantation. First, the human liver is decellularized. The resulting acellular liver is lyophilised and comminuted to a particulate form. This ECM powder is then made into a solution, which is finally used in combination with a gelling agent to make the ECM-hydrogels.

\subsubsection{Histology, Immunohistochemistry and Immunofluorescence}

Human liver ECM-hydrogels, previously fixed in 10\% formalin (Leica Biosystems), were first washed in distilled water and then dehydrated in a series of ethanol (Acquascience) and xylene baths, followed by embedding in paraffin (Leica Biosystems). The samples were sliced into $5 \mu \mathrm{m}$ sections using a microtome (Leica Biosystems Leica RM2035). All sections were then immersed in xylene baths (Acquascience) for at least 5 minutes, and ethanol baths (Acquascience) for at least 2 minutes and then rinsed in tap water.

\subsubsection{Histology}

Sections were then stained as follows:

Haematoxylin and Eosin (H\&E): Sections were first stained with Harris Haematoxylin (Leica Biosystems) for 10 minutes followed by washing in tap water for 5 minutes. Next, sections were stained with Eosin (Leica Biosystems) for 3 minutes, followed by washing in tap water for 5 minutes. The sections were then quickly dehydrated in 
ethanol and then immersed in xylene bath (Acquascience) until mounted with DPX (Leica Biosystems).

Picro-Sirius Red $(S R)$ : Sections were stained with Picro-Sirius Red (R.A.Lamb; Cl35780) for approximately 20 minutes. The sections were then quickly dehydrated in ethanol and then immersed in xylene bath (Acquascience) until mounted.

Elastic Van Gieson (EVG): Sections were soaked in $0.5 \%$ potassium permanganate for 5 minutes and washed in washed in distilled water for 1 minute followed by immersion in $1 \%$ oxalic acid solution for 1 minute and washed in distilled water. Section were quickly treated with absolute alcohol and stained with neat Miller's Elastic - (R.A. Lamb, LAMB/080D) for 2 hours. Section were washed with industrially methylated spirits (IMS) (Thermo Fisher Scientific) and then washed in tap water for 5 minutes. In the final step, sections were stained with Van Gieson (Leica Biosystems) for 5 minutes. The sections were then quickly dehydrated in ethanol and then immersed in xylene bath (Acquascience) until mounted.

All sections were mounted with DPX (Leica Biosystems) and finally cover slipped. Images were captured with an Axiocam lcC5 using Zeiss Axiovision (verison 4.8.2)

\subsubsection{Immunohistochemistry}

Samples slides derived from fixed acellular ECM-hydrogels were analysed through immunohistochemistry in order to confirm the retention of the most abundant ECM proteins such as Collagen I, Collagen III, Laminin and Fibronectin. To do this, slides were incubated inside an oven placed at $37^{\circ} \mathrm{C}$ for 30 minutes in a solution composed of $0.5 \%$ Trypsin (MP Biomedical) / 0.5\% Chymotrypsin (Sigma) / 1\% Calcium Chloride $(\mathrm{BDH})$ in $10 \%$ Tris buffered saline (TBS). This was followed by a washing step in 10\% TBS at pH 7.6 with $0.04 \%$ Tween-20 (Sigma) for 5 minutes. Slides were incubated for 5 minutes in blocking solution (Novocastra). This was followed by an incubation step for 1 hour with the primary antibodies diluted in blocking solution as described in table 2. 
(Rabbit pAb to coll1, Abcam, ab34710)

\section{Collagen III}

(Rabbit pAB to coll3, Abcam, ab7778)

Fibronectin

(Mouse mAb to fibronectin, Millipore, MAB1937)

\section{Laminin}

(Mouse mAb to laminin a5-chain, Millipore MAB1924)
$1: 500$

$1: 100$

$1: 200$

Table 2. List of primary antibodies used for the immunohistochemical staining of ECMhydrogels.

Slides were then first incubated for 25 minutes in Novolink ${ }^{\mathrm{TM}}$ post primary (Novocastra) and then for other 25 minutes in Novolink ${ }^{\mathrm{TM}}$ polymer solution to avoid non-specific staining (Novocastra). Slides were then developed with Novolink ${ }^{\mathrm{TM}}$ 3,3' di-amino-benzidine (Novocastra) and finally counterstained for 5 minutes using Mayer's Hematoxylin (Sigma).

All sections were mounted with DPX (Leica Biosystems) and cover slipped. Images were taken with an Axiocam IcC5 using Zeiss Axiovision (verison 4.8.2).

For the optimization of the ECM-hydrogel immunohistochemical staining was performed by Mr Andrew Rennie Hall, Department of Cellular Pathology, UCL Medical school Royal Free Campus.

For the in vivo application of ECM-hydrogels, gels were bioengineered with HepG2 and implanted into the omentum of immunodeficient mice. Samples were retrieved from the abdominal area of the mice and fixed in 10\% formalin for 48 hours. Samples were paraffin-embedded, sliced into $5 \mu \mathrm{m}$ sections by using a microtome (Leica Biosystems Leica RM2035) and analysed through immunohistochemistry staining by Mustapha Najimi, Université Catholique de Louvain, Laboratory of Paediatric Hepatology \& Cell Therapy, Institute of Experimental and Clinical Research, Belgium. 
Prior to Ku80 staining, heat-induced epitope retrieval was performed in DAKO target retrieval buffer (DAKO, S1699) for 1 hour, followed by blocking with TBST containing $5 \%$ normal goat serum (Sigma, G9023). Sections were incubated overnight at $4{ }^{\circ} \mathrm{C}$ with Ku80 primary antibody (Cell Signalling, CST-2180; diluted 1:800 with blocking solution). Samples were incubated with secondary antibody (Invitrogen, A-11008; diluted 1:600 with blocking solution) and visualized using an EVOS microscope [215].

\subsubsection{Immunofluorescence}

Bioengineered ECM-hydrogels with HepG2 were fixed in 10\% formalin, paraffin embedded and sliced into $5 \mu \mathrm{m}$ sections. Slides were washed twice (5 minutes each time) with $1 \mathrm{X}$ PBS and fixed with $10 \%$ formalin in PBS for 10 minutes. This was followed by a washing step in PBS (3 times, 5 minutes). Cells were permeabilised with $1 \%$ triton X-100 in PBS for 5 minutes followed by a washing step with PBS (3 times, 5 minutes). Next, in order to avoid non-specific bindings, slides were incubated in a blocking solution ( $1 \%$ bovine serum albumin and $0.1 \%$ sodium azide in PBS) for 30 minutes. The first step of staining was incubation for 30 minutes with Alexa Fluor ${ }^{\text {TM }}$ 594 Phalloidin (Thermo Fisher Scientific; diluted 1:40 with the blocking solution). This was followed by washing steps with PBS (2 times, 5 minutes). Finally, slides were air dried and mounted with VECTASHIELD Antifade Mounting Medium with DAPI (Vector laboratories). Samples were then visualised under fluorescent microscope (Nikon model U-III equipped with Nikon mercury lamp).

Samples of bioengineered ECM-hydrogels with Induced Pluripotent Stem Cells (iPSC) were fixed in formalin $10 \%$ for 20 minutes and washed with two times with PBS $1 \mathrm{X}$ (Sigma). Samples were incubated for 1 hour with blocking solution composed of $1 \%$ Triton-X100 (Sigma) and 3\% BSA (Sigma) in PBS 1X, followed by a washing step with PBS 1X. Samples were incubated overnight with the following primary antibodies diluted 1:100 in blocking solution for A1AT (DAKO, A0012), ALB (R\&D, 188835) and HNF4a (Santa Cruz, sc6556). Samples were washed three times PBS 1X for 20 minutes each and then incubated for 2 hours with the following secondary antibodies diluted 1:500 in blocking solution for Alexa Fluor 488 (Invitrogen, A-11055) and Alexa Fluor 568 (Invitrogen, A-10037). This was followed by washing steps with 1X PBS (3 
times, 20 minutes). Nuclei were stained for 30 minutes with Hoechst 33258 (Sigma) diluted 1:5000 in PBS 1X, followed by a washing step with 1X PBS (3 times, 20 minutes).

For both DAPI and Phalloidin staining the bioengineered ECM-hydrogels were incubated for 1 hour with of Phalloidin TRICT (Sigma; diluted 1:200 in blocking solution). This was followed by washing steps with 1X PBS (3 times, 20 minutes). Samples were incubated for 20 minutes with of Hoechst 33258 (Sigma) diluted 1:5000 in PBS 1X, followed by a washing step with 1X PBS (3 times, 20 minutes).

Immunofluorescence staining of ECM-hydrogel was performed by Dr Rute Da Costa Tomaz, Wellcome Trust MRC Cambridge Stem Cell Institute.

\subsubsection{Scanning Electron Microscopy (SEM)}

Human liver ECM-hydrogels were first fixed at $4^{\circ} \mathrm{C}$ for 48 hours in $2.5 \%$ glutaraldehyde followed by washing steps in $0.1 \mathrm{M}$ phosphate buffer and then cryoprotected for 2 hours using a solution composed of $25 \%$ sucrose, $10 \%$ glycerol in 0.05 M PBS. ECMhydrogels were then snap frozen with liquid nitrogen and fractured at $-160^{\circ} \mathrm{C}$. Samples were then soaked in the aforementioned cryoprotectant solution in order to thaw at room temperature. ECM-hydrogels were washed in $0.1 \mathrm{M} \mathrm{PBS}$ and fixed at $4^{\circ} \mathrm{C}$ for 2 hours using $1 \%$ OsO4 in $0.1 \mathrm{M}$ PBS. This was followed by a washing step in $0.1 \mathrm{M}$ PBS and a dehydration step using ethanol-based solutions. Samples were dried using $\mathrm{CO}_{2}$ and then mounted on aluminium pin stubs with the help of adhesive carbon tapes. Last, fractured ECM-hydrogels were coated with a $2 \mathrm{~nm}$ layer of $\mathrm{Au} / \mathrm{Pd}$. Images were captured using a 7401 FEG scanning electron microscope (Jeol, USA).

ECM-hydrogels for scanning electron microscopy were processed by $\mathrm{Dr}$ Claire Crowley, UCL Institute of Child Health. 


\subsubsection{Rheological evaluation of the stiffness of human liver ECM hydrogel}

Rheological experiments were performed using a TA Instruments Discovery Hybrid HR-1 Rheometer using an 8mm parallel plate and a Peltier plate Steel to control the sample temperature. ECM-hydrogels containing Agarose $0.25 \%(\mathrm{w} / \mathrm{v})$ were prepared following protocol 3 (acetic acid and sonication) as described before in the paragraph 2.3.3. For the temperature ramp test, samples were loaded into the rheometer with the Peltier plate Steel set at $4{ }^{\circ} \mathrm{C}$. Before starting the experiment, the temperature was set at $40{ }^{\circ} \mathrm{C}$, with an increasing temperature rate of $1^{\circ} \mathrm{C}$ every 30 seconds, while a constant frequency was set at $0.998 \mathrm{~Hz}(6.28 \mathrm{rad} / \mathrm{s})$. The moduli of the samples were analysed during the increase in temperature from $4{ }^{\circ} \mathrm{C}$ up to $40^{\circ} \mathrm{C}$.

The viscoelastic profile of the ECM-hydrogels was measured by performing a frequency sweep test between $0.019 \mathrm{~Hz}(0.12 \mathrm{rad} / \mathrm{s})$ and $38.9 \mathrm{~Hz}(244.5 \mathrm{rad} / \mathrm{s})$ at 25 ${ }^{\circ} \mathrm{C}$. Results were analysed using software Trios V3.3.

Rheological measurements of ECM-hydrogels were performed with the help of Anastasia Papadopolou at UCL, Department of Engineering.

\subsubsection{Maintenance of hepatic cells in culture}

Human hepatoblastoma cells (HepG2) were used for this study. HepG2 is an immortalized cell line consisting of human liver carcinoma cells, derived from liver tissue of a 15-year-old Caucasian male [216] and kindly provided by Prof Clare Selden ILDH. HepG2 cells were cultured in MEM Medium (Gibco Thermo Fisher Scientific) supplemented with 10\% Foetal Bovine Serum (FBS; Gibco Thermo Fisher Scientific), 2mM L-glutamine (200mM Gibco Thermo Fisher Scientific), 1\% Sodium Pyruvate (100X Gibco Thermo Fisher Scientific), 1\% Non-Essential Amino Acids (100X Gibco Thermo Fisher Scientific) and 1\% 1X Antibiotic-Antimycotic (100X Gibco Thermo Fisher Scientific). HepG2 cells were cultured in a humidified incubator at $37{ }^{\circ} \mathrm{C}$ with 
$5 \% \mathrm{CO}_{2}$. Once cells reached $\sim 75 \%$ confluency, cells were trypsinised using $0.25 \%$ Trypsin-EDTA (Thermo Fisher Scientific) and passaged at a split ratio of 1:3.

\subsubsection{ECM hydrogel sterilisation}

Human liver ECM-hydrogels were individually sterilised in $2 \mathrm{ml}$ safe safe-lock tubes (Eppendorf) by adding $1.5 \mathrm{ml}$ of a sterilant solution composed of $0.1 \%$ peracetic acid (Sigma-Aldrich) and 4\% ethanol (Fisher Chemical) in deionised water. Hydrogels were exposed to the sterilising solution for 30 minutes in an orbital shaker (Staurt). This was followed by a washing step by adding to each ECM hydrogel $1.5 \mathrm{ml}$ of sterile 1X HBSS (Thermo Fisher Scientific) for 15 minutes on an orbital shaker. Sterile ECM-hydrogels were then transferred to a 48 well plate and $1.4 \mathrm{ml}$ of culture media was added to each ECM hydrogel. Samples were kept overnight inside the incubator at $37^{\circ} \mathrm{C}$ with $5 \%$ $\mathrm{CO}_{2}$ in order to verify their sterility prior to the re-seeding with human cells.

\subsubsection{D cell cultures in human liver ECM-hydrogels}

HepG2 cells and Induced Pluripotent Stem Cells (iPSC) were used to perform the experiments described in this Chapter. For both cell types, ECM-hydrogels were left overnight in media as mentioned above. After confirming their sterility microscopically, ECM-hydrogels were transferred into a 96 well plate.

HepG2 cells were trypsinised and re-suspended at a concentration of 0.5 million cells/20 $\mu \mathrm{l}$ culture medium. Twenty $\mu \mathrm{l}$ of cell resuspension was added on top of each ECM hydrogel and samples were placed in a humidified incubator at $37^{\circ} \mathrm{C}$ with $5 \%$ $\mathrm{CO}_{2}$. After 2 hours from the cell seeding, $120 \mu \mathrm{l}$ of culture medium was added to each ECM hydrogel and samples were placed in a humidified incubator at $37{ }^{\circ} \mathrm{C}$ with $5 \%$ $\mathrm{CO}_{2}$. The day after the seeding, ECM-hydrogels were transferred to a 48 well plate and $1.4 \mathrm{ml}$ of culture medium was added to each sample. During the experiment cell culture medium was changed every 3 days, such as day $4,7,10$, etc. During cell 
culture, cell supernatants were collected and stored in $-20^{\circ} \mathrm{C}$ freezer for further cell functionality evaluation.

Acellular liver cubes and hydrogels which contained solely agarose $0.25 \%$, used as $3 \mathrm{D}$ controls in the experiments described in this Chapter, were sterilised, re-seeded and cultured following the same methodology used for ECM hydrogels.

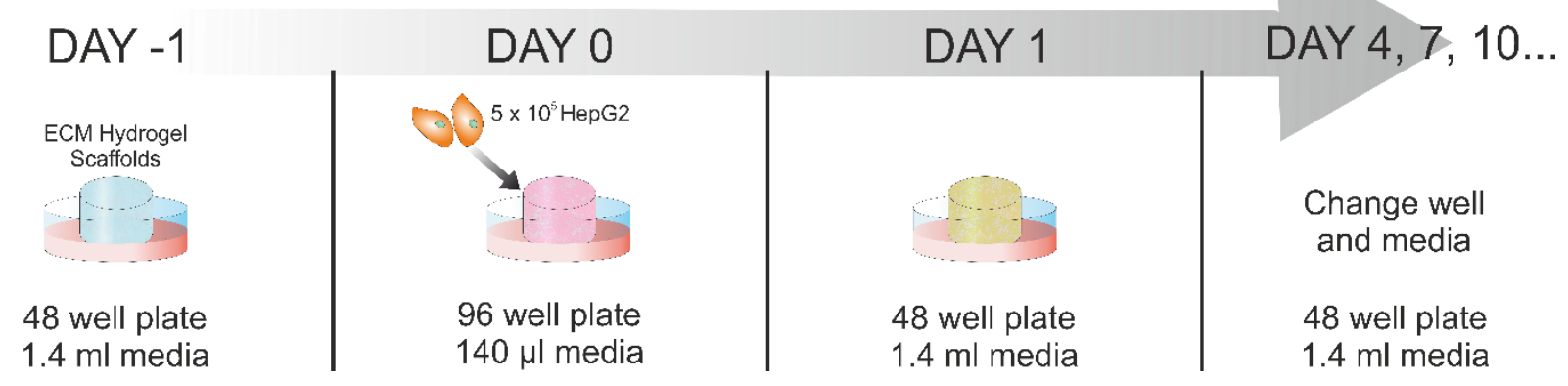

Figure 9. Schematic representation of the timeline for 3D cell culture.

Induced Pluripotent Stem Cells (iPSC), kindly provided by Prof Ludovic Vallier (Cambridge Stem Cell Institute and the Wellcome Sanger Institute), were re-seeded in ECM-hydrogels. To do this, IPSCs obtained from different stages of differentiation towards hepatocytes-like cells were re-seeded on ECM-hydrogels (see Figure 10 below) 1) IPSCs previously differentiated for 12 days on 2D fibronectin coated plastic (these cells are considered to be at the hepatoblast stage) were cultured for 18 days on ECM-hydrogels; 2) IPSCs previously differentiated for 23 days on 2D fibronectin coated plastic (these cells are considered to be immature hepatocytes) were cultured for 7 days on ECM-hydrogels; and 3) IPSCs previously differentiated for 30 days on 2D fibronectin coated plastic were cultured for 7 days on ECM-hydrogels (these cells are considered to be mature hepatocytes) (Figure 10).

For all IPSCs conditions, cells cultured in 2D were detached using Accutase (SigmaAldrich) and re-suspended at a concentration of 0.5 million cells $/ 20 \mu$ culture medium. Twenty $\mu$ l of cell resuspension was added on top of each ECM hydrogel and samples were placed inside a humidified incubator at $37{ }^{\circ} \mathrm{C}$ with $5 \% \mathrm{CO}_{2}$. After 2 hours from the cell seeding, $120 \mu$ of culture medium was added to each ECM hydrogel and 
samples were placed inside a humidified incubator at $37{ }^{\circ} \mathrm{C}$ with $5 \% \mathrm{CO}_{2}$. The day after the seeding, ECM-hydrogels were transferred to a 48 well plate and $1.4 \mathrm{ml}$ of culture medium was added to each sample. During the experiment cell culture medium was changed every 2 days, such as day 3,5,7,9,11 etc. IPSCs were cultured in Hepatozyme Medium (Gibco), supplemented with , 2\% CD Lipids (Gibco), 2mM Lglutamine (200mM Gibco Thermo Fisher Scientific), 2\% Non-Essential Amino Acids (100X Gibco Thermo Fisher Scientific),1\% 1X Antibiotic-Antimycotic (100X Gibco Thermo Fisher Scientific), 0.14\% Insulin (Gibco), 0.1\% Transferrin (Gibco), 0.1\% OncostatinM (R\&D 295-OM) and 0.1\% HGF (Peprotech 100-39). During cell culture, cell supernatants were collected and stored in $-20{ }^{\circ} \mathrm{C}$ freezer for further cell functionality evaluation.

Differentiation of IPSCs towards hepatocyte-like cells was evaluated through Albumin secretion analysis, gene expression and immunofluorescence

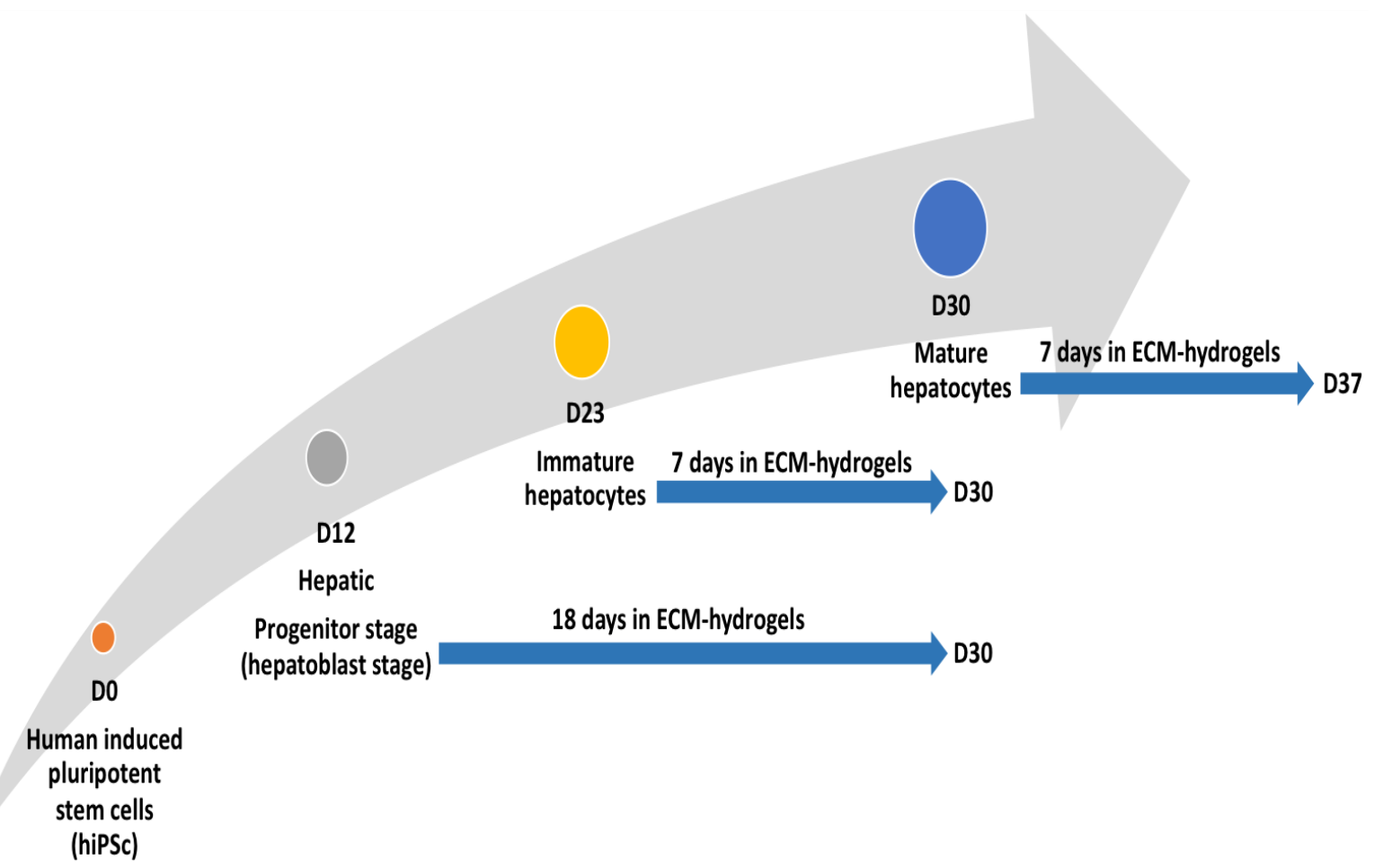

Figure 10. Schematic representation of IPSCs seeded on ECM-hydrogels. IPSC previously differentiated for 12 days on 2D fibronectin coated wells (hepatoblast stage) were cultured for 18 days on 3D ECM-hydrogels. IPSC previously differentiated for 23 days on 2D and (immature hepatocytes stage) were then cultured for 7 days on 3D ECM-hydrogels. IPSC previously differentiated for 30 days on $2 \mathrm{D}$ (mature hepatocytes stage) were cultured for 7 days on 3D ECM-hydrogels. 


\subsubsection{Alamar blue viability test}

Alamar blue assay is based on the reduction of resazurin, a blue and weakly fluorescent compound, to resorufin, a pink and fluorescent compound. Only the alive cells are able to produce resorufin and therefore the intensity of the detected fluorescence signal is proportional to the number of living cells. AlamarBlue ${ }^{\circledR}$ (Thermo Fisher Scientific) cell viability reagent $10 \mathrm{X}$ was first diluted 1:10 with cell culture medium and then $1.4 \mathrm{ml}$ of resulting solution was added to each bioengineered ECMhydrogels followed by a 4 hours incubation in a humidified incubator at $37^{\circ} \mathrm{C}$ with $5 \%$ $\mathrm{CO}_{2}$. Quadruplicates of each sample (200 $\mu \mathrm{l}$ per well) were analysed in a 96 opaque black well plate. Microplate Reader (Infinite M200 PRO Multimode Microplate reader, Tecan, Switzerland) was used to measure the absorbance at $570 \mathrm{~nm}$.

\subsubsection{Human Albumin ELISA}

Content of secreted albumin in HepG2 cell supernatants was quantified by performing Human Albumin ELISA assay (Human Albumin ELISA kit ABCAM). HepG2 supernatants, collected at different time points during cell culture, were diluted 1:60 with Diluent Solution provided in the kit. Fifty $\mu \mathrm{l}$ of diluted cell supernatants and Albumin Standard Solutions were added and incubated in a 96 well plate for 1 hour followed by 5 washes with Washing Buffer. Fifty $\mu$ l of 1 X Biotinylated Albumin Antibody was then added in each well and washed after 30 minutes. Next, 1X SP Conjugate antibody was added and washed after 30 minutes. Fifty $\mu \mathrm{l}$ of Chromogen Substrate Solution was added in each well and incubated for 25 minutes. Last, $50 \mu \mathrm{l}$ of Stop Solution was used to block the reaction until the colour of the samples turned from blue to yellow. Absorbance signal related to albumin content was read at $450 \mathrm{~nm}$ using the plate reader (FLUOstar Omega BMG Labtech).2.3.12. RNA extraction, reverse transcription and gene expression analysis. 


\subsubsection{RNA extraction}

\subsubsection{RNA extraction}

RNeasy Plant Kit (Qiagen) was employed to extract RNA from the HepG2 and IPSCs cells grown in ECM-hydrogels. Lysis Buffer (Qiagen) containing $1 \%$ of $\beta$ mercaptoethanol (Sigma-Aldrich) was first added to the previously snap frozen samples followed by vigorously pipetting to further disrupt the samples. The main reason for using the Plant kit was the necessity to remove the agarose present in the hydrogels in order to achieve the maximum yield of RNA. This step was performed by using the QIAshredder column provided by the manufacturer. As indicated in the protocol provided by the manufacturer, subsequent steps were performed using spin columns and different ethanol-based buffers. RNA extracted from ECM-hydrogels was measured at $260 \mathrm{~nm}$ with the spectrophotometer (NanoDrop, ThermoScientific).

\subsubsection{Complementary DNA (cDNA) synthesis}

Complementary cDNA reverse transcription was performed using high Capacity cDNA Reverse Transcription Kit (Applied Biosystems). The extracted RNA was diluted in RNase free water (Qiagen) in order to obtain the same RNA concentration in all the samples. Subsequently, $10 \mu \mathrm{l}$ of the samples' RNA was added in independent PCR microtubes and then mixed with $10 \mu \mathrm{l}$ of Master Mix, which composition is shown in table 3. The reverse transcription was performed using the 2720 Thermal Cycler (Applied Biosystems) following the program described in table 4. 


\begin{tabular}{|l|l|}
\hline RT Master Mix reagents & Volume $(\boldsymbol{\mu L})$ \\
\hline 10X RT Buffer & 2 \\
\hline 25X dNTPs Mix (100 mM) & 0.8 \\
\hline 10X RT Random Primers & 2 \\
\hline MultiScribe RT & 1 \\
\hline RNase Inhibitor & 1 \\
\hline Nuclease Free $\mathrm{H}_{\mathbf{2}} \mathbf{0}$ & 3.2 \\
\hline
\end{tabular}

Table 3. RT Master Mix composition.

\begin{tabular}{|l|l|}
\hline Step 1 & 10 minutes at $25^{\circ} \mathrm{C}$ \\
\hline Step 2 & 120 minutes at $37^{\circ} \mathrm{C}$ \\
\hline Step 3 & 5 minutes at $85^{\circ} \mathrm{C}$ \\
\hline Step 4 & Hold at $4^{\circ} \mathrm{C}$ \\
\hline
\end{tabular}

Table 4. Steps of Reverse transcription program using 2720 Thermal Cycler.

\subsubsection{Quantitative Real-Time PCR (RT- qPCR)}

The obtained cDNA was first diluted in RNase free water to get a final concentration of $2 \mathrm{ng} / \mathrm{\mu l}$. Ten $\mathrm{ng}$ of cDNA from each sample was added into a Fast Optical 96-well TaqMan PCR Plate (MicroAmp, Applied Biosystems) and then mixed with $15 \mu \mathrm{l}$ of 
qPCR Master Mix, which composition is described in table 5. Last, the PCR Plate was inserted in ABI 7500 Fast Real Time PCR System (Applied Biosystems) to perform 40 cycles of cDNA amplification (table 6).

Comparative $\mathrm{Ct}$ method was used to analyse the relative expression of the investigated genes (table 7) using Glyceraldeyde-3-phosphate dehydrogenase (GAPDH) as housekeeping gene [217].

\begin{tabular}{|l|l|}
\hline RT-qPCR master mix reagents & Volume $(\mu \mathrm{L})$ \\
\hline TaqMan Gene Assay-FAM & 1 \\
\hline TaqMan Univ PCR MM (2X), w/UNG & 10 \\
\hline $\mathbf{H}_{2} \mathbf{O}$ (nuclease free Water) & 4 \\
\hline
\end{tabular}

Table 5. RT-qPCR master mix composition.

\begin{tabular}{|c|c|}
\hline Step 1 & 2 minutes at $50^{\circ} \mathrm{C}$ \\
\hline Step 2 & 10 minutes at $95^{\circ} \mathrm{C}$ \\
\hline Step 3 & 5 minutes at $95^{\circ} \mathrm{C}$ for 40 cycles \\
\hline Step 4 & 11 minutes at $60^{\circ} \mathrm{C}$ \\
\hline
\end{tabular}

Table 1. Program used for Quantitative Real-Time PCR (RT- qPCR) using ABI 7500 Fast Real Time PCR System. 


\begin{tabular}{|l|l|l|}
\hline Gene & NCBI Ref. Seq. & ID Number \\
\hline GAPDH & NM_001256799.2 & Hs02786624_g1 \\
\hline AFP & NM_001134.2 & Hs01040598_m1 \\
\hline ALB & NM_000477.5 & Hs00910225_m1 \\
\hline HNF4A & NM_000457.4 & Hs00230853_m1 \\
\hline CYP1A2 & NM_000761.4 & Hs00167927_m1 \\
\hline NR1H4 & NM_001206977.1 & Hs01026590_m1 \\
\hline CSP1 & NM_001122633.2 & Hs00157048_m1 \\
\hline OTC & NM_000531.5 & Hs00166892_m1 \\
\hline
\end{tabular}

Table 2. List of TaqMan assays on demand used (LifeScience Technologies).

\subsubsection{In vivo biocompatibility of bioengineered ECM-hydrogels}

This study was carried out in accordance with the recommendations in the UK Animal (Scientific Procedures) Act 1986. The UK Home Office approved the study protocol (licence number70/2716). The work was approved by the London-Hampstead NRES ethics committee REC reference 13/LO/0171 [218]. The surgical implantation and explanation procedures were performed by Dr Federico Scottoni at the UCL, Institute of Child Health.

The in-vivo biocompatibility of ECM-hydrogels bioengineered with HepG2 cells was investigated by employing 3 male NOD SCID mice. To do this, 0.5 million were seeded on each ECM hydrogel and cultured for 7 days prior the implantation into the omentum of the immunodeficient mice (Figure 11).

During the surgical procedure, the abdominal area of the mice was first shaven and Videne antiseptic solution (Ecolab) was applied to clean the skin. Mice were 
anesthetised using Isoflurane followed by an abdominal incision to implant the ECM hydrogel wrapped into the omentum sheet. After three weeks, mice were euthanized to allow the retrieval of the implants that were immediately fixed in formalin $10 \%$ in order to be analysed through histology and immunohistochemistry.

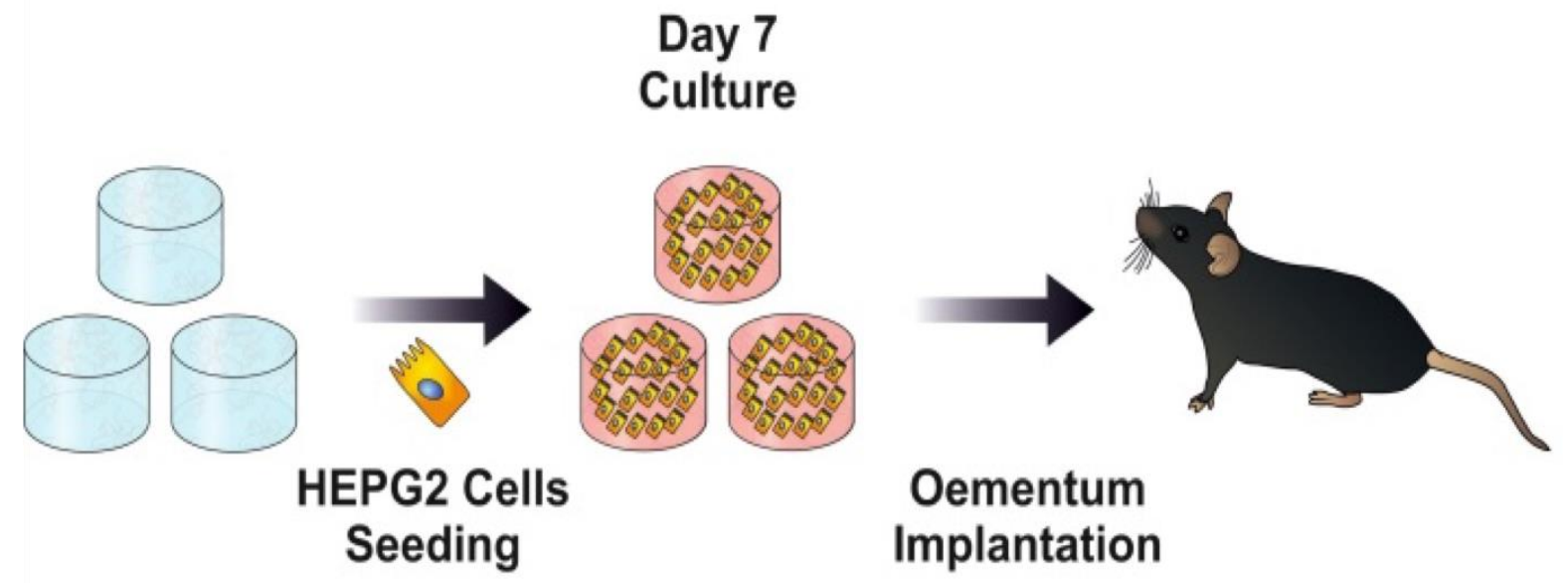

Figure 11. Schematic overview for HepG2 bioengineered ECM-hydrogel prior their surgical implantation into the omentum of immunodeficient mice.

\subsubsection{Statistics and data analysis}

Paired t-test was performed to evaluate statistical differences in the experimental results, using the error bars to represent the standard deviation. SPSS v21 software was used to perform the statistical analysis. 


\subsection{Results}

\subsubsection{Development of Human Liver ECM-hydrogels}

\subsubsection{Protocol 1}

As previously mentioned, the initial protocol (Protocol 1) tested for the development of human liver ECM-hydrogels, was aimed to compare the efficiency of a previously published protocol for porcine liver [186] with the decellularization of human livers. After 72 hours ECM proteins were not completely digested by pepsin, as some particles were visible present. Additionally, the resultant human liver ECM solution did not self-assemble into hydrogel after incubation for 1 hour at $37^{\circ} \mathrm{C}$ (Figure 12).

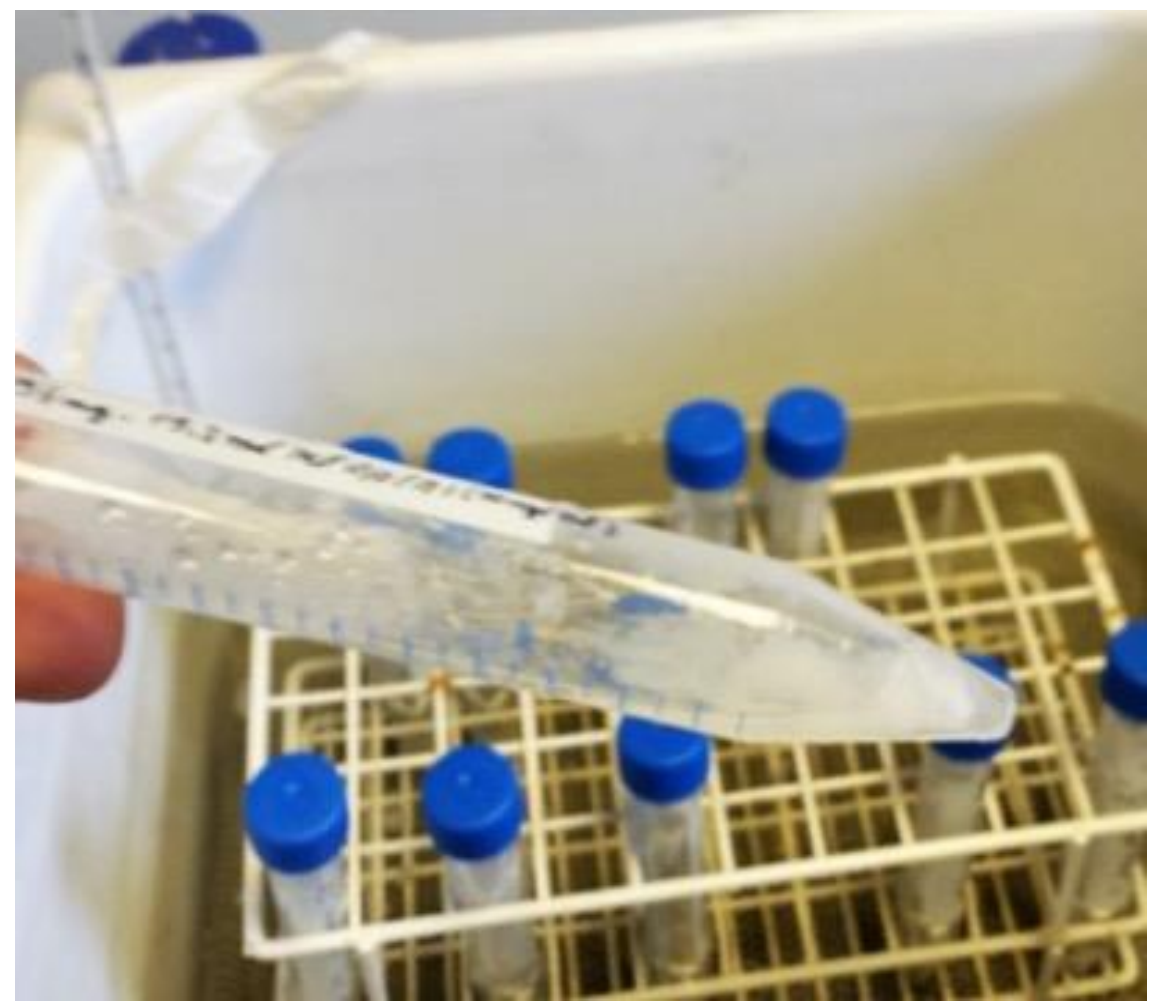

Figure 12. Outcome of Protocol 1 for the development of human liver ECM-hydrogels. ECM proteins were not completely digested and the resultant ECM solution did not self-assemble into a hydrogel after incubation at $37^{\circ} \mathrm{C}$. 


\subsubsection{Protocol 2}

To overcome previous described complications of protocol 1, protocol 2 was developed in which experimental steps remained the same but the pepsin concentration was doubled, in order to improve the ECM proteins digestion, thus the final ECM protein concentration was increased from $6 \mathrm{mg} / \mathrm{ml}$ to $12 \mathrm{mg} / \mathrm{ml}$. After 72 hours of pepsin digestion, very few particles were still visible present in the ECM solution, indicating that the ECM digestion was more successful. However, the resultant ECM solution did not self-assemble into a hydrogel after incubation for 1 hour at $37^{\circ} \mathrm{C}$ (Figure 13).

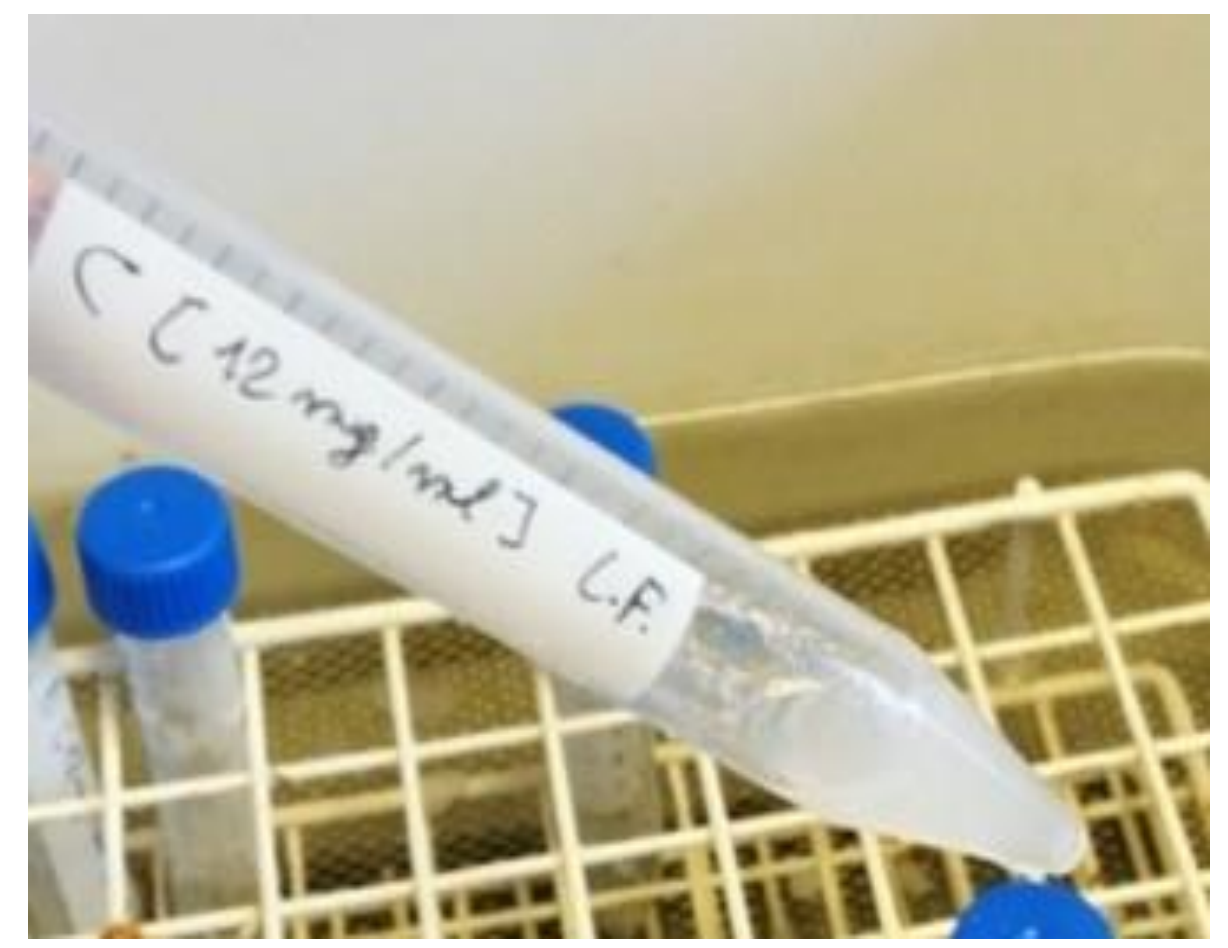

Figure 13. Outcome of Protocol 2 for the development of human liver ECM-hydrogels. ECM proteins were almost completely digested but resultant ECM solution did not self-assemble into hydrogel after incubation at $37^{\circ} \mathrm{C}$.

\subsubsection{Protocol 3}

Collagen and other ECM proteins are more soluble in organic acid, like acetic acid, compared to inorganic acids [219]. For this reason, in this $3^{\text {rd }}$ attempt human liver ECM 
powder was solubilised in acetic acid $0.5 \mathrm{M}$ for 3 days and the solution was sonicated to improve ECM components solubilisation. This novel protocol allowed complete suspension of human liver ECM components. In addition, final ECM concentration was diluted to $4 \mathrm{mg} / \mathrm{ml}$. Important, the resultant human liver ECM solution did not selfassemble into hydrogel after incubation for 1 hour at $37^{\circ} \mathrm{C}$ as shown in Figure 14 .

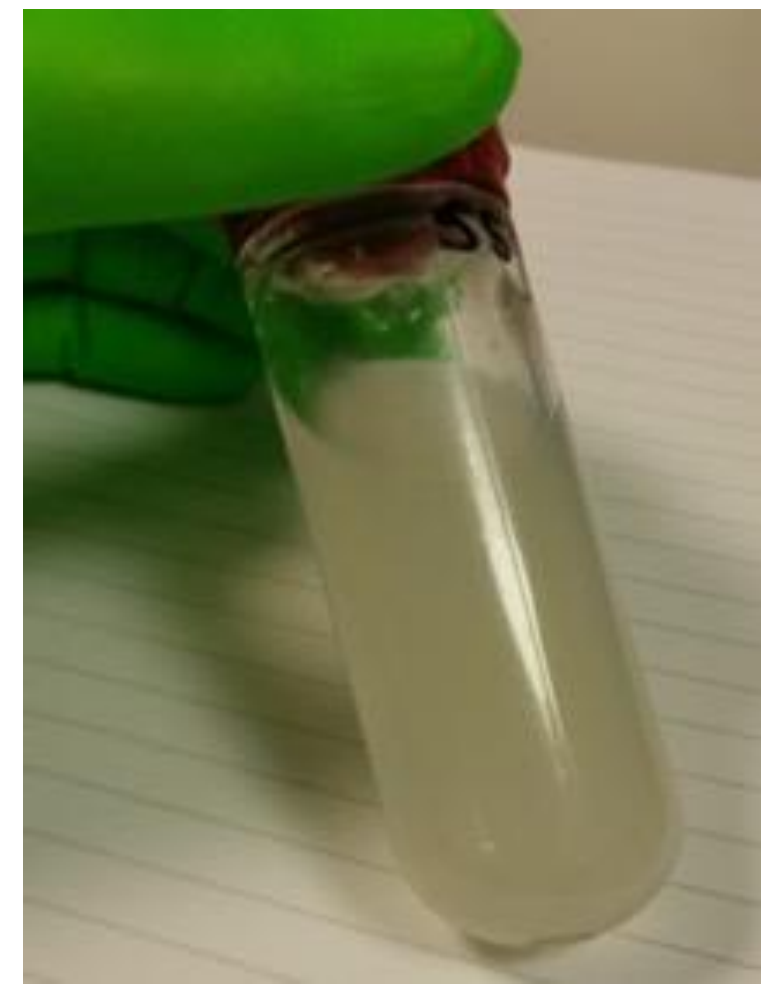

Figure 14. Outcome of Protocol 3 for the development of human liver ECM-hydrogels. By performing this protocol 3 the human liver ECM powder was completely solubilised but the resultant ECM solution did not self-assemble into hydrogel after incubation at $37^{\circ} \mathrm{C}$.

\subsubsection{Gelation of Human liver ECM solution with gelling agents}

Gelation of human liver ECM solution prepared following protocol 3 i.e. with acetic acid and sonication, but without the use of pepsin, was achieved by employing gelling agents such as agarose and agar. After preparing human liver ECM solution following protocol 3, agarose and agar powders were heated using microwave until complete solubilisation in $1 \mathrm{X}$ PBS to obtain $2 \% \mathrm{w} / \mathrm{v}$ stock solutions. Resultant gelling agent solutions were cooled down and lastly, different volumes of agar or agarose solutions 
$(0.125-1 \% \mathrm{v} / \mathrm{v})$ were used to gel the human liver ECM solution. Figure 15 showed the macroscopic view of the ECM-hydrogels prepared from decellularised human liver ECM solution, gelled with different concentrations of $(a-e)$ agarose or $(f-l)$ agar. $(a, f)$ $0.125 \%$, (b,g) $0.25 \%$, (c-h) $0.5 \%$, (d-i) $0.75 \%$, and (e,l) $1.0 \%$ of gelling agents. Except for agar at $0.125 \%$, all other gelling agent concentrations allowed to gel the human liver ECM solution.
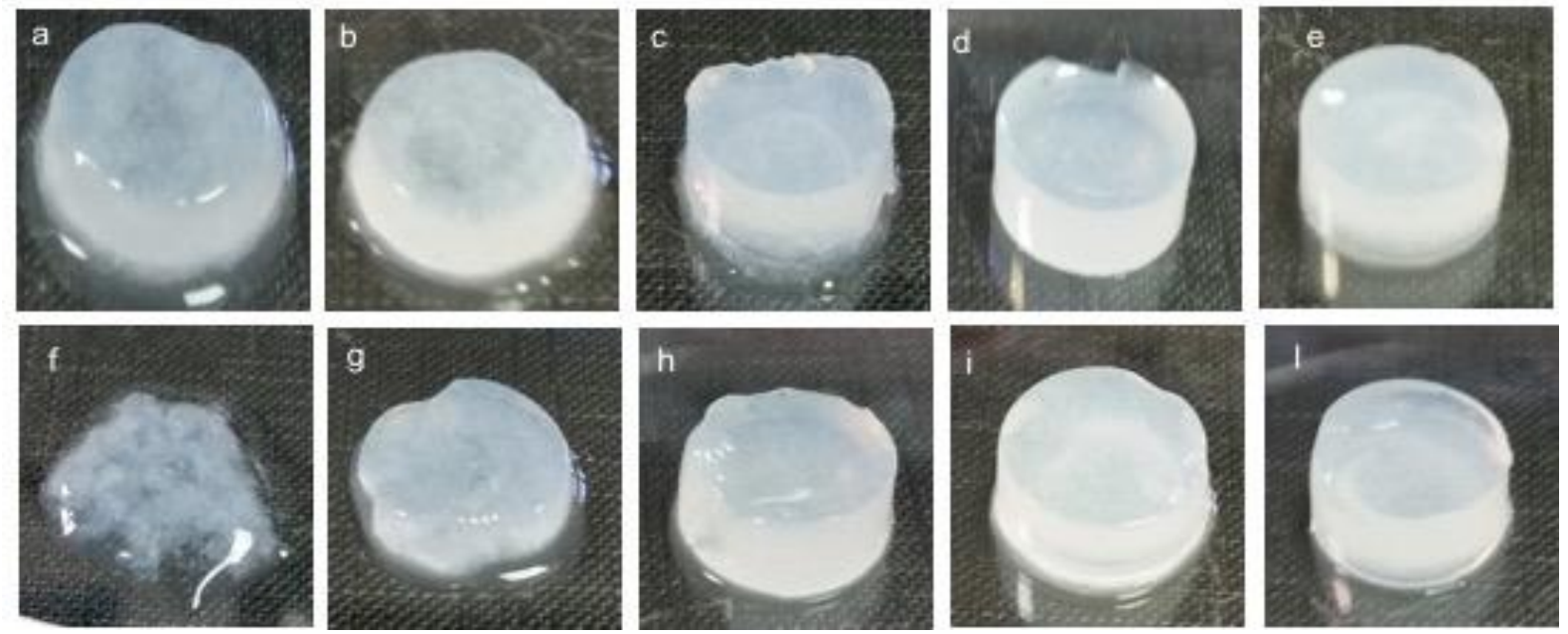

Figure 15. Macroscopic view of ECM-hydrogels prepared from decellularised human liver ECM solution, gelled with different concentrations of (a-e) agarose or (f-l) agar. $(a, f) 0.125 \%$, $(b, g) 0.25 \%$, (c-h) $0.5 \%$, (d-i) $0.75 \%$, and (e,l) $1.0 \%$ gelling agent.

\subsubsection{Characterisation of Human Liver ECM Hydrogel}

\subsubsection{Histological and Immunohistochemistry analysis of human ECM- hydrogels}

As shown in Figure 16, retention of the most abundant ECM proteins into the ECMhydrogels was investigated by histological staining for total collagen obtained by Picro Sirius Red staining (f) and elastin by Van Gieson staining (e), and by immunohistochemistry for collagen I (a), collagen III (b), laminin (c) and fibronectin (d). Resultant images confirmed retention and the presence of networks made by these 
ECM proteins after gelation of the ECM solution. Images were taken from human liver ECM hydrogel containing agarose $0.25 \%(\mathrm{v} / \mathrm{v})$.
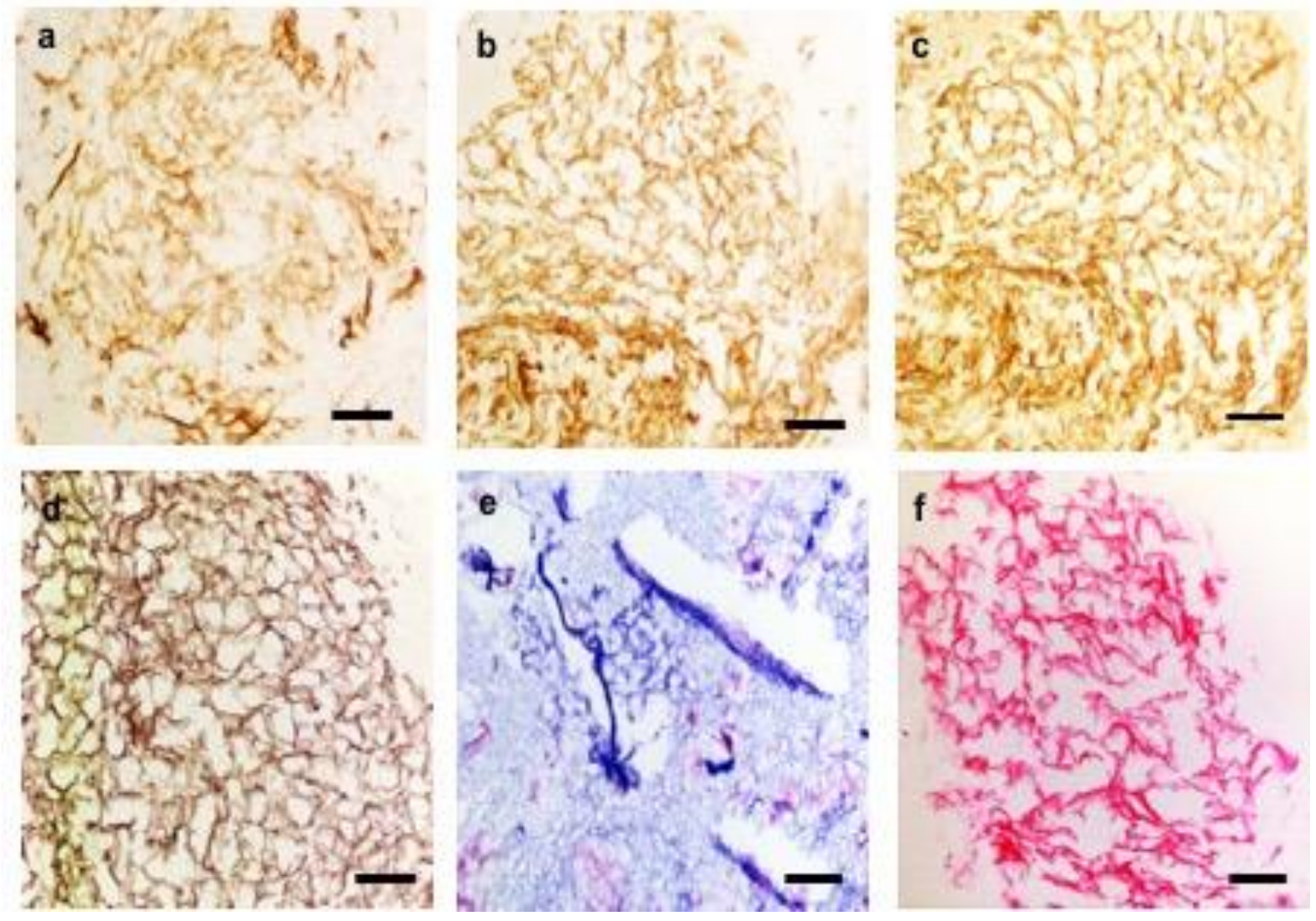

Figure 16. Retention of the most abundant ECM proteins within the ECM-hydrogels has been investigated by immunohistochemistry for (a) collagen-I, (b) collagen-III, (c) laminin, (d) fibronectin, (e) elastin Van Gieson staining and (f) Picro Sirius Red staining. Images confirm the presence of networks made by these ECM proteins. Scale bar $50 \mu \mathrm{m}$.

\subsubsection{Scanning Electron Microscopy (SEM) of human liver ECM-hydrogels}

Ultrastructure of human liver ECM-hydrogels was characterised by Scanning Electron Microscopy (SEM). As shown in Figure 17, SEM images at different magnification confirmed the presence of nanofibrous structures into the ECM-hydrogels. In addition, SEM images of ECM-hydrogels containing $0.25 \%(\mathrm{v} / \mathrm{v})$ of agarose confirmed a proper porosity. This would allow, or favour, in future experiments the diffusion of oxygen and nutrients during cell culture. 


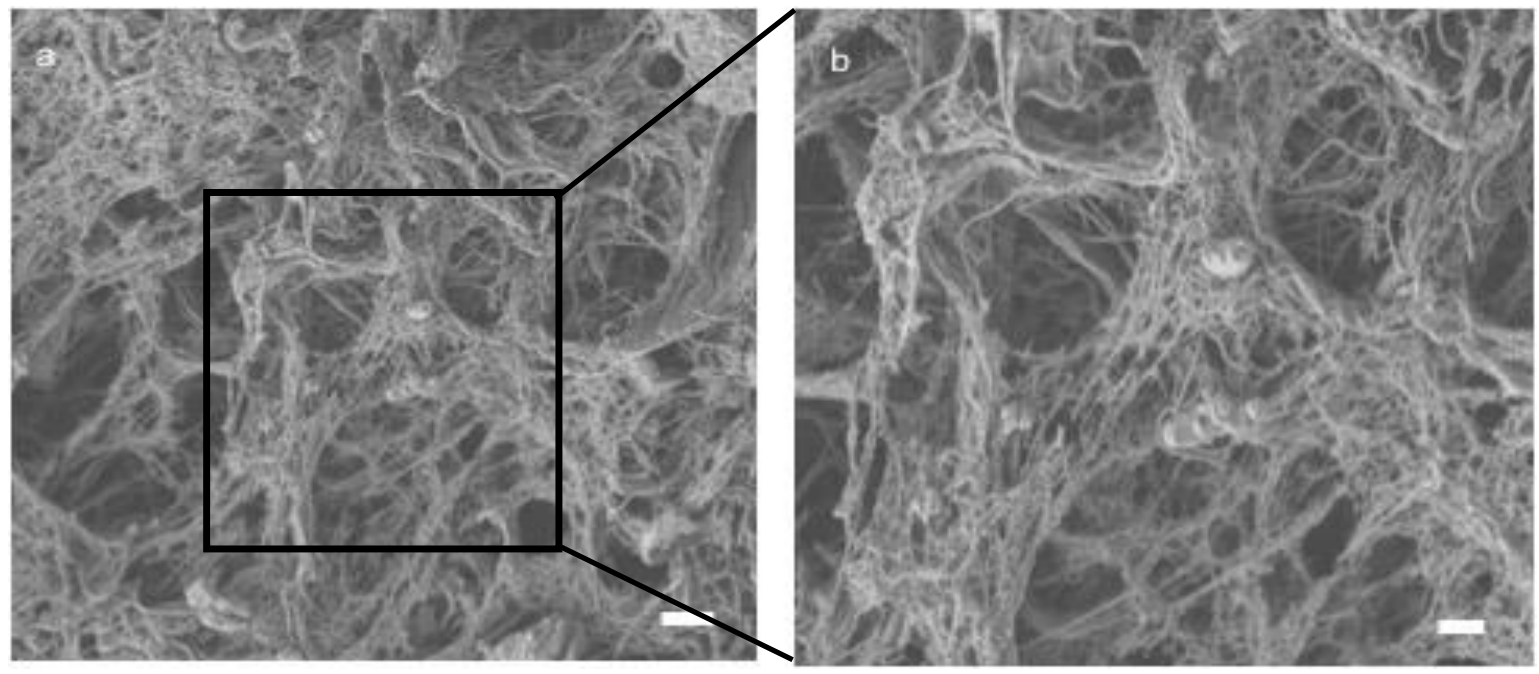

Figure 17. Scanning Electron Microscopy (SEM) images of ECM-hydrogels containing $0.25 \%$ (v/v) agarose. Scale bars (a) $10 \mu \mathrm{m}$, (b) $1 \mu \mathrm{m}$.

\subsubsection{Rheological analysis of human liver ECM-hydrogels}

The temperature ramp test was performed to evaluate if stiffness (storage modulus) and viscosity (loss modulus) were affected by temperature. As shown in Figure 18 (a), in the analysed temperature range $\left(4-40^{\circ} \mathrm{C}\right)$, stiffness and viscosity are constant, indicating that ECM hydrogel is very stable due to hydrogen bonds. Furthermore, the storage modulus results 10 fold higher than the loss modulus, suggesting a solid-like behaviour of the hydrogel at any analysed temperature. Important, stiffness of ECMhydrogels containing $0.25 \%(\mathrm{v} / \mathrm{v})$ agarose was found to be $1.2 \mathrm{kPa}$ at $37^{\circ} \mathrm{C}$.

Injectability of ECM-hydrogels containing agarose $0.25 \%(\mathrm{v} / \mathrm{v})$ was investigated by performing the frequency sweep test between $0.019 \mathrm{~Hz}(0.12 \mathrm{rad} / \mathrm{s})$ and $38.9 \mathrm{~Hz}$ $(244.5 \mathrm{rad} / \mathrm{s})$ at $25^{\circ} \mathrm{C}$. As shown in Figure $18(\mathrm{~b})$, dynamic viscosity decreased while increasing applied stress, showing a similar behaviour of commercially available injectable materials such as collagen type I injectable gel [113]. 


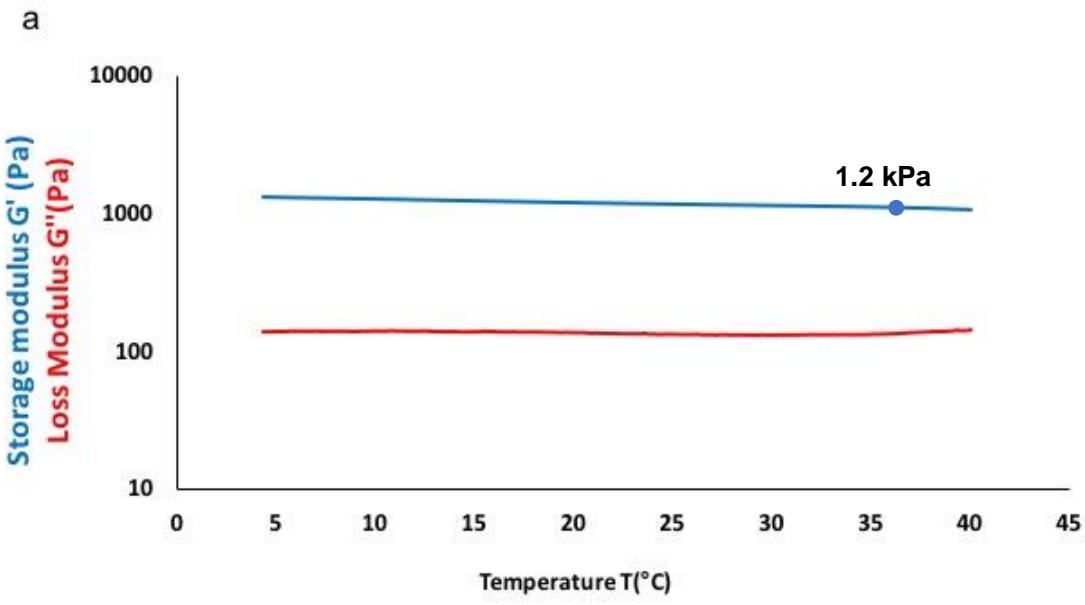

-Storage modulus $\mathrm{Pa} \quad$ - Loss modulus $\mathrm{Pa}$

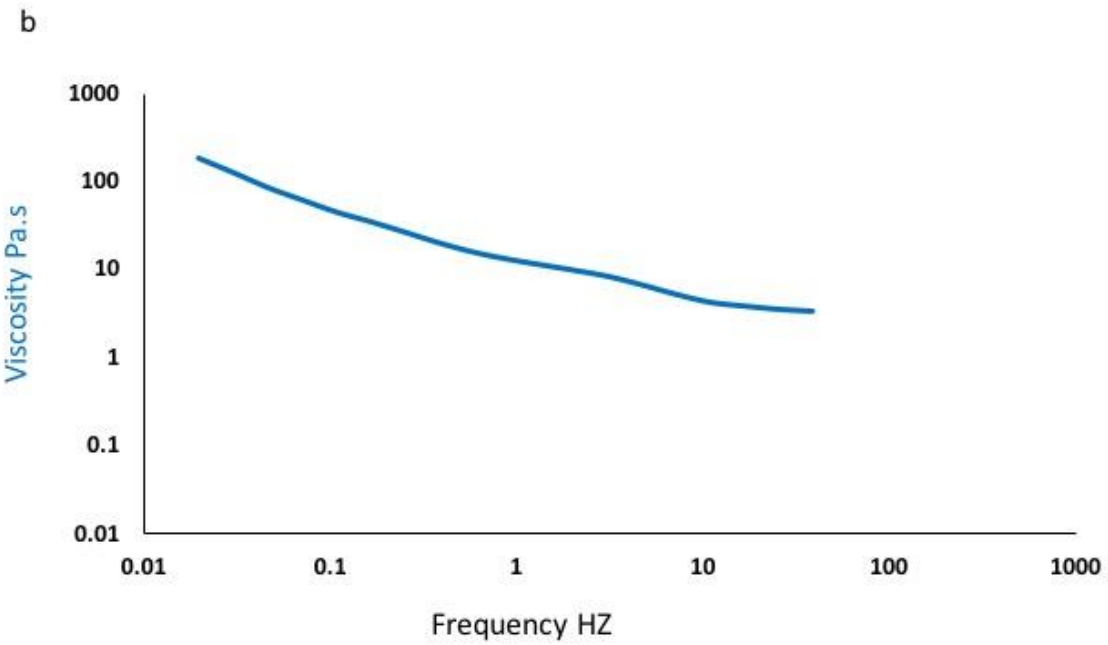

Figure 18. Rheological characterisation of ECM hydrogel containing $0.25 \%(\mathrm{v} / \mathrm{v})$ of agarose. (a) Temperature ramp test and (b) frequency sweep test. $n=3$.

2.4.3. Assessing the in vitro biocompatibility of human liver ECMhydrogels

\subsubsection{Effect of gelling agent on HepG2 functionality}

In vitro biocompatibility of the developed human liver ECM-hydrogels was investigated by seeding 0.5 million of HepG2 cells on ECM-hydrogels ( $n=4$ for each condition) containing $0.125 \%(\mathrm{v} / \mathrm{v})$ or $0.25 \%(\mathrm{v} / \mathrm{v})$ of agarose and $0.25 \%(\mathrm{v} / \mathrm{v})$ or $0.50 \%(\mathrm{v} / \mathrm{v})$ of agar. These four conditions were selected because containing the lowest gelling agent 
concentration (except for agar $0.125 \%$ but this concentration was not able to gel the ECM solution thus used as negative control). Functionality of HepG2 seeded on these 4 conditions was investigated by performing the human albumin ELISA assay. As shown in Figure 19, the albumin quantification of bioengineered HepG2 hydrogels showed a time dependent increase in albumin production/secretion between day 3 and day 7 for all conditions investigated except for hydrogels with agar $0.25 \%$.

The highest cell functionality and lowest standard deviation has been obtained by ECM-hydrogels containing $0.25 \%(\mathrm{v} / \mathrm{v})$ of agarose so this condition was selected to carry on in the next sets of experiments.

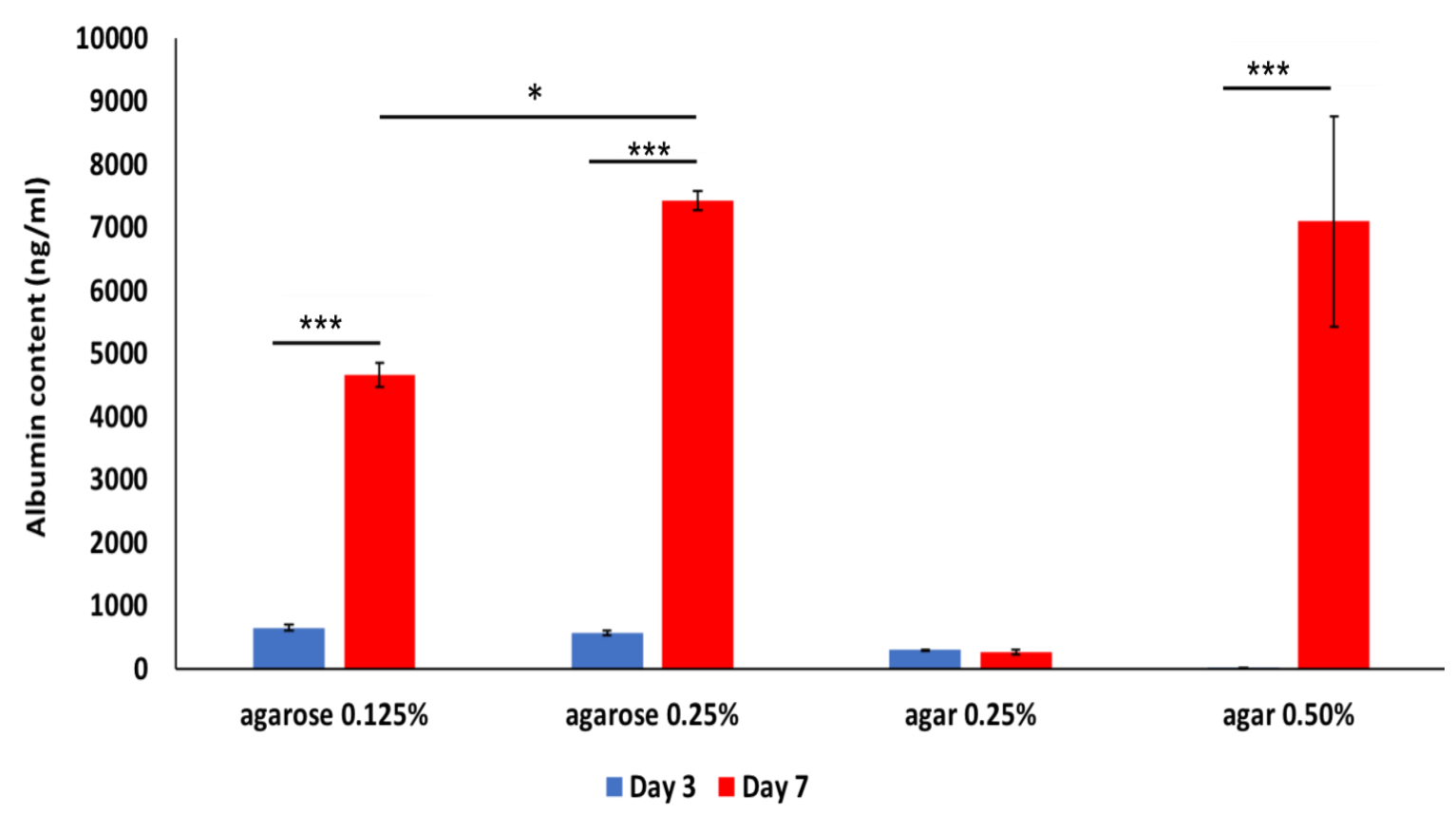

Figure 19. Albumin secretion quantified of HepG2 bioengineered ECM-hydrogels containing $0.125 \%(\mathrm{v} / \mathrm{v})$ and $0.25 \%(\mathrm{v} / \mathrm{v})$ of agarose and $0.25 \%(\mathrm{v} / \mathrm{v})$ and $0.50 \%(\mathrm{v} / \mathrm{v})$ of agar. $\mathrm{n}=4$ for each condition. ${ }^{*} p<0.05,{ }^{* * *} p<0.001$.

\subsubsection{Evaluation of the importance of ECM proteins for cell attachment, viability and functionality}

The importance of ECM proteins for cell attachment, viability and functionality, was evaluated by seeding HepG2 cells on ECM-hydrogels containing agarose $0.25 \%(\mathrm{v} / \mathrm{v})$ 
and on hydrogels that were composed solely by agarose $0.25 \%$. Scaffolds $(n=4$ for both conditions) were repopulated with 0.5 million HepG2 and cultured up to 10 days. As shown in Figure 20, attachment and viability of seeded HepG2 cells was investigated by histological-and immunofluorescence staining. (a) Haematoxylin and eosin staining of ECM-hydrogel in agarose $0,25 \%$ at 10 days of cell culture showed that HepG2 cells attached, migrated into the scaffold and were organised in clusters. No cells were found in hydrogels composed only by agarose $0.25 \%$. Cell morphology was analysed by performing (b) fluorescent DAPI (4',6-diamidino-2-phenylindole) to stain nuclei and (c) fluorescent phalloidin staining to capture the structure of the Factin cytoskeleton merged with DAPI confirmed the retention of the natural hexagonal shape of the HepG2 cell. Human Albumin ELISA was performed to quantify HepG2's functionality into bioengineered scaffolds and showed a time dependent increase of secreted human albumin in the ECM-hydrogels and absence of albumin in the hydrogels made of solely agarose (graph d). At day 4 of cell culture a minimum amount of albumin was detected from hydrogels composed by only agarose while at day 7 and 10 albumin was not detected. These data indicated that few cells attached to these hydrogels during the first days of culture but died during longer cell culture (day 7 and day 10$)$. 


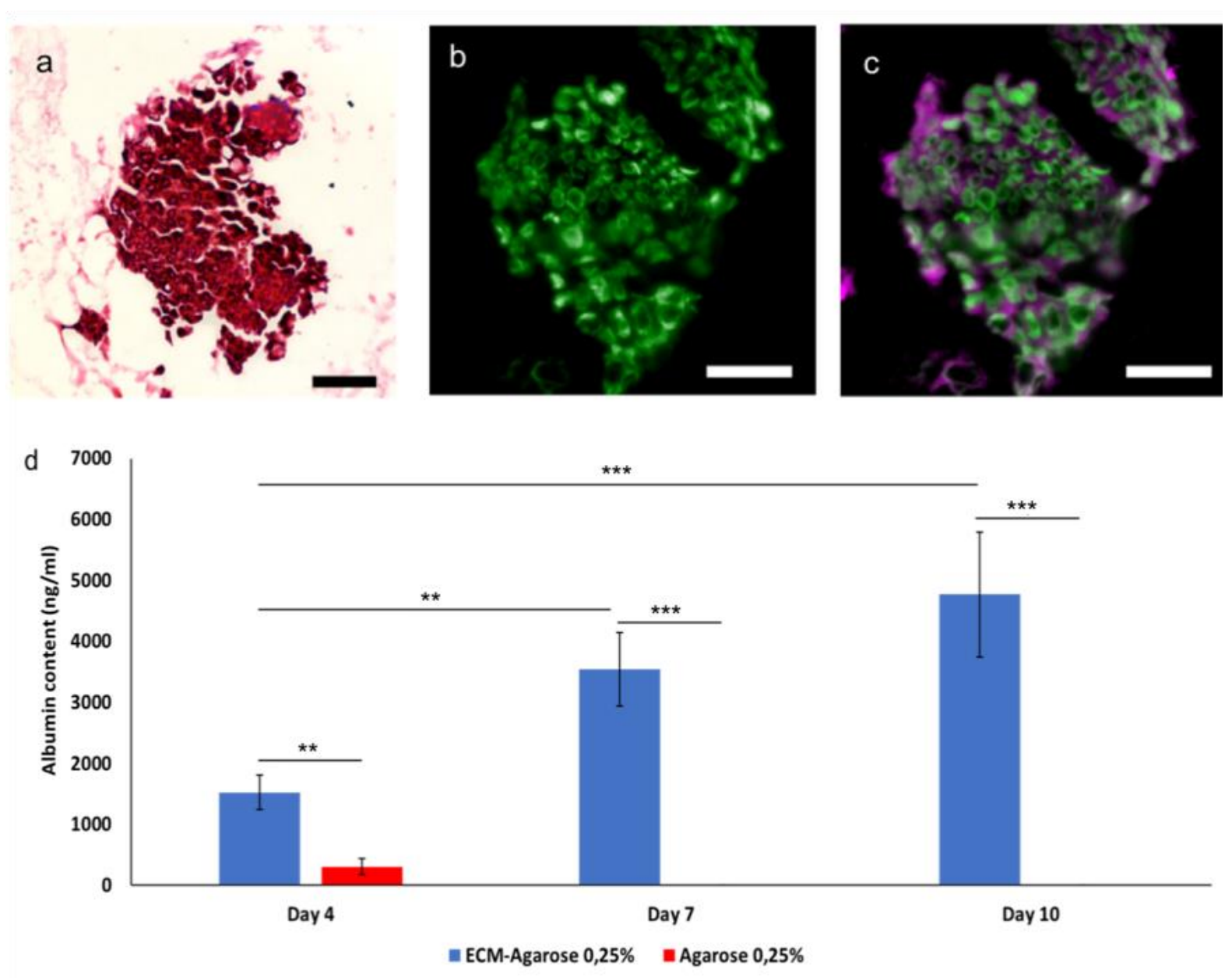

Figure 20. (a) Haematoxylin and eosin staining of ECM-hydrogel in agarose 0,25\% at 10 days of cell culture. Cell morphology analysis by performing (b) fluorescent DAPI (4',6-diamidino-2phenylindole) and (c) fluorescent Phalloidin staining merged with DAPI. (d) Human Albumin ELISA to evaluate HepG2 functionality into bioengineered hydrogels. Scale bar (a-c) $50 \mu \mathrm{m}$. ${ }^{* *} p<0.01,{ }^{* * *} p<0.001$. $n=4$ per condition.

\subsubsection{Long-term in-vitro culture of HepG2}

HepG2 cells $\left(0.5 \times 10^{6}\right)$ were seeded on ECM-hydrogels gel with agarose $0,25 \%(v / v)$ $(n=4)$ in order to evaluate cell viability and albumin production. As shown in Figure 21 (a), viability of HepG2 cells was evaluated by performing Alamar Blue assay and resulting graph showed a steady state in cell number from day 7 to day 13 while from day 13 to day 19 cell numbers significantly increased. Human Albumin ELISA was performed to evaluate cell functionality into ECM-hydrogels during cell culture. Resultant albumin secretion graph (Figure $21 \mathrm{~b}$ ) from bioengineered hydrogels showed a reduction in albumin secretion from day 7 to day 13 followed by a statistically significant increase from day 13 to 19 days. 

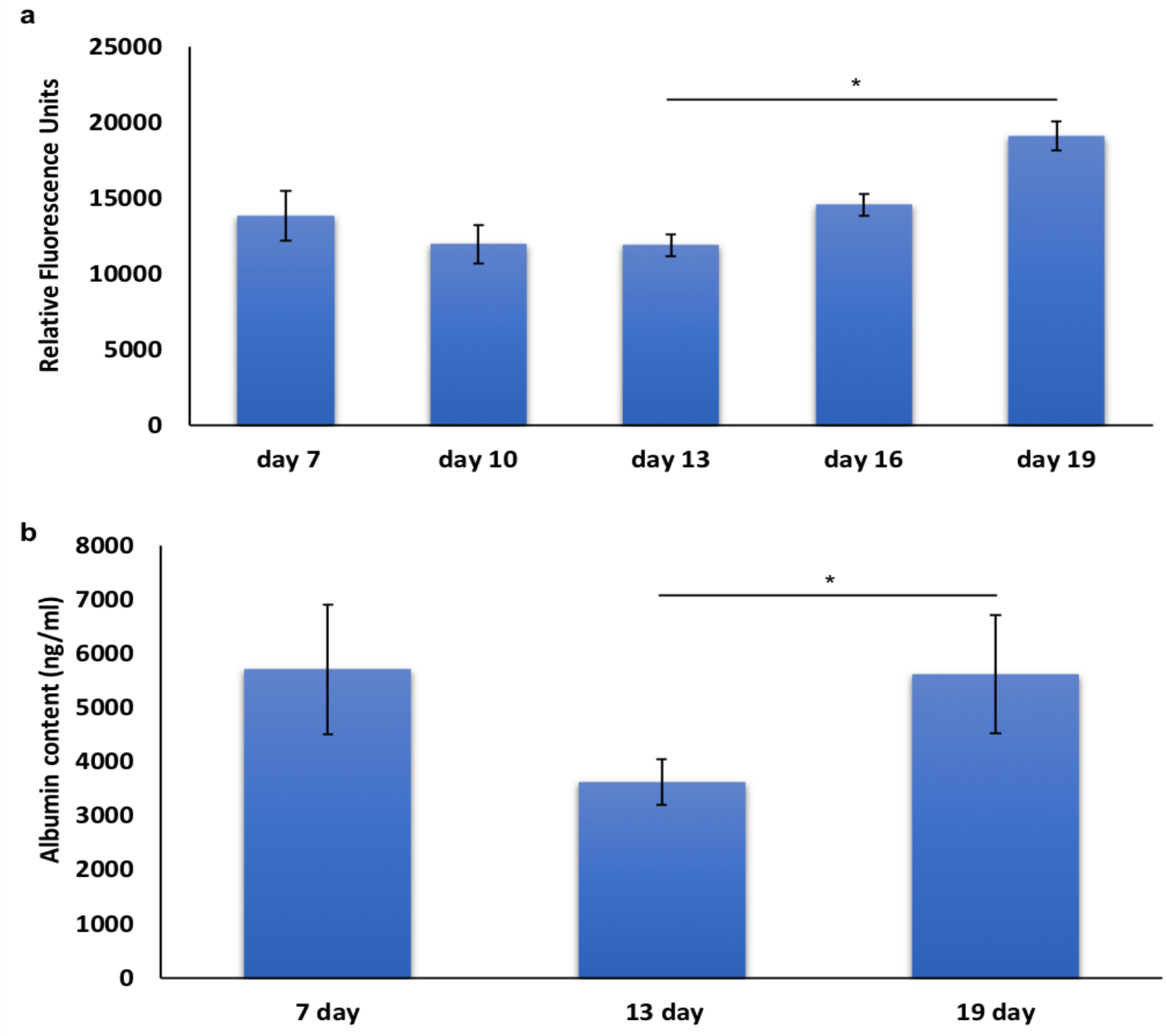

Figure 21. (a) Alamar blue viability assay and (b) HepG2 albumin secretion. * $p<0.05 . n=4$ per condition.

\subsubsection{Comparison in gene expression and functionality of HepG2 cultured in ECM-hydrogels and in acellular liver cubes}

Albumin secretion and gene expression of HepG2 cell culture were compared between ECM-hydrogels (Figure 22 a) $(n=4)$ and HepG2 cultured in acellular liver cubes (Figure 22 b) ( $n=4)$. The latter was employed as an internal three-dimensional platform control (65). Haematoxylin and eosin staining of ECM-hydrogel containing agarose $0,25 \%$ and $3 D$ liver cube (Figure $22 \mathrm{c}$ and d) after 13 days of cell culture confirmed attachment of the cells onto both types of 3D constructs. Albumin secretion (Figure $22 \mathrm{e}$ ) shows higher HepG2 cell functionality in acellular liver cubes in the first 
4 days of cell culture. Conversely, metabolic activity of HepG2 cells cultured in ECMhydrogels is shown to be higher from day 4 to day 13. Gene expression analysis (Figure $22 \mathrm{f}-\mathrm{I}$ ) confirmed a higher metabolic profile of HepG2 cultured in ECMhydrogels compared to those HepG2 cultured in acellular liver cubes. Indeed, gene expression showed that HepG2 cells seeded on ECM-hydrogels expressed a higher level of (f) albumin, (i) NR1H4 and (m) CPS1, and an equal level of (h) AFP, (h) HNF4A and (I) CYP1A2. In line with other publications [220], the expression of OTC was found undetectable in both HepG2 bioengineered ECM-hydrogels and acellular liver cubes.
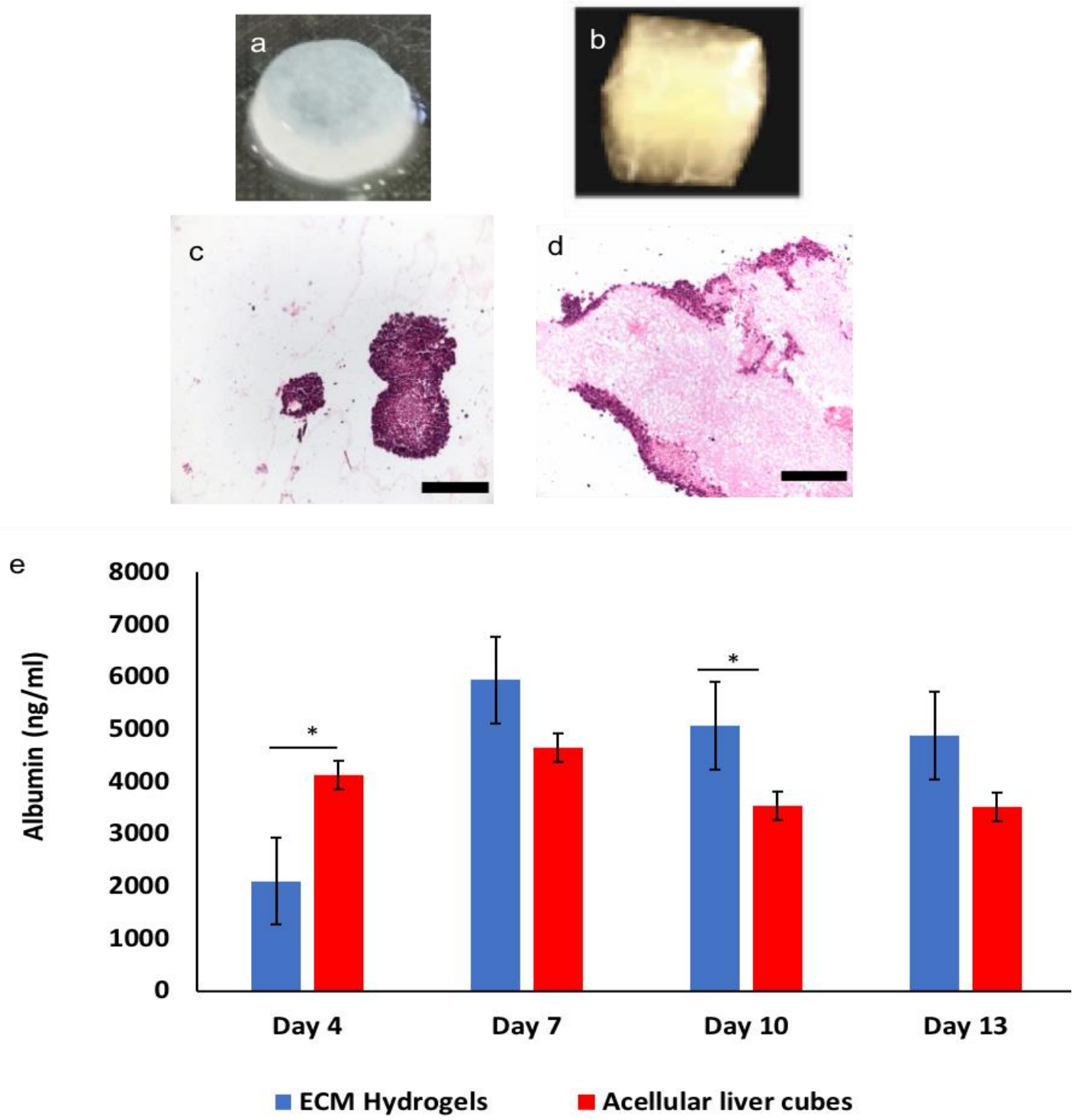

Figure 22 (a-e). (a,b) Macroscopic view of (a) human liver ECM hydrogel and (b) human liver acellular liver cube. (c,d) Hematoxylin and eosin staining of (c) ECM-hydrogel agarose $0,25 \%$ and (d) 3D liver cube after 13 days of cell culture. (e) Human albumin quantification graph 
f
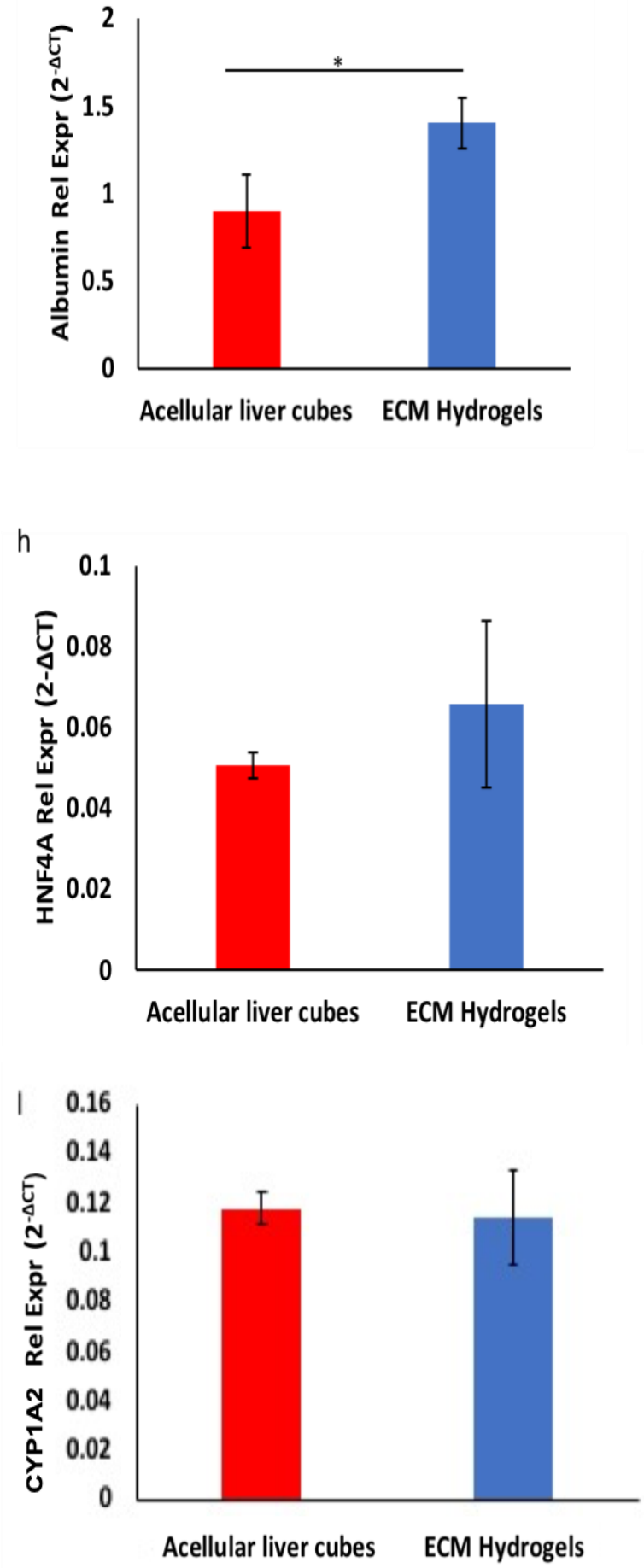

g
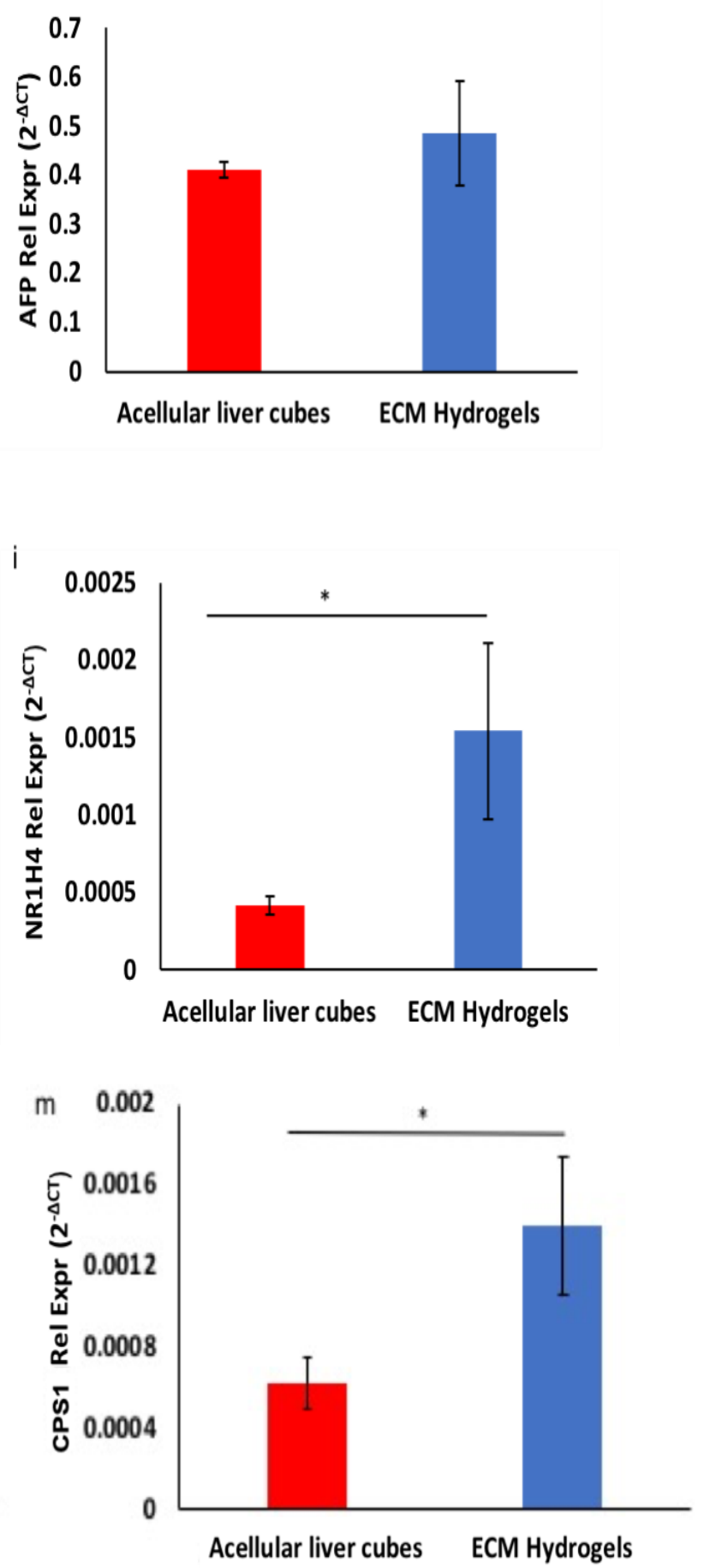

Figure $23(\mathrm{f}-\mathrm{m})$. (f-m) Gene expression analysis of HepG2 cells seeded on ECM-hydrogels and acellular liver cubes. HepG2 cells express a higher level of (f) albumin, (i) NR1H4 and (m) CPS1, and an equal level of (h) AFP, (h) HNF4A and (I) CYP1A2. (a,b) Scale bar $100 \mu \mathrm{m}$. ${ }^{*} \mathrm{p}<0.05 . \mathrm{n}=4$ per condition. 


\subsubsection{Investigating the in vivo biocompatibility of ECM-hydrogels}

Investigating the in vivo biocompatibility test of bioengineered ECM-hydrogels with HepG2 was a key experiment. More specifically to analyse whether this human liver ECM-based model has the potential to keep the cells alive after implantation and improve their engraftment in the host tissue.

Figure 23 shows HepG2 cells cultured for 7 days on ECM-hydrogels before the bioengineered samples $(n=3)$ were implanted into the omentum of immunodeficient mice (NOD SCID mice). The choice of implantation site was based on the characteristic vasculature system of this area [221] in order to supply the cells with oxygen and nutrients and therefore to maintain them alive after implantation.

Samples were implanted in mice for a total of 3 weeks. The surgical implantation (Figure 23) and explanation (Figure 24) procedures were performed by Dr Federico Scottoni at the UCL, Institute of Child Health.
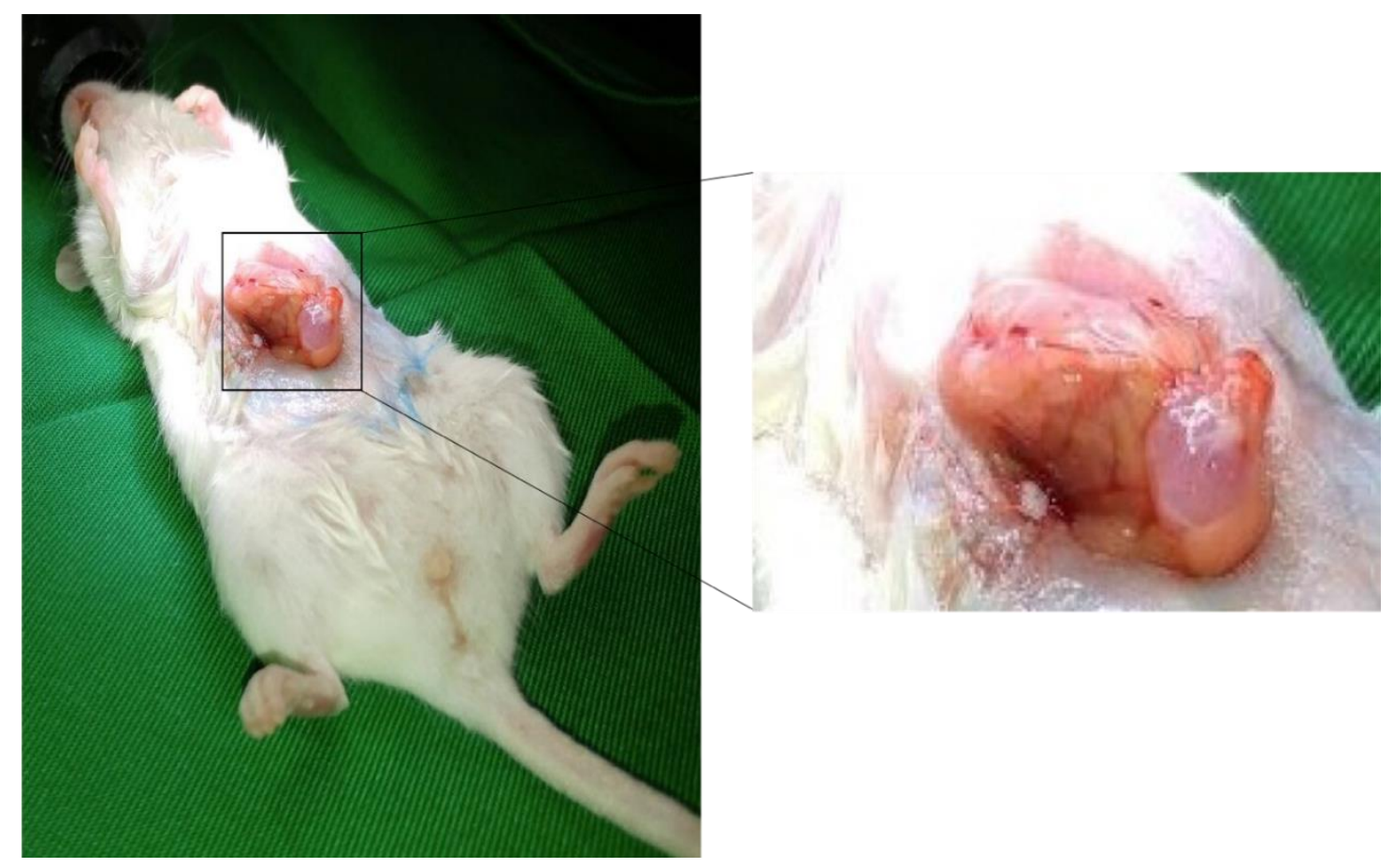

Figure 24. Surgical procedure for the implantation of bioengineered ECM-hydrogels with HepG2 into the omentum of immunodeficient mice. 


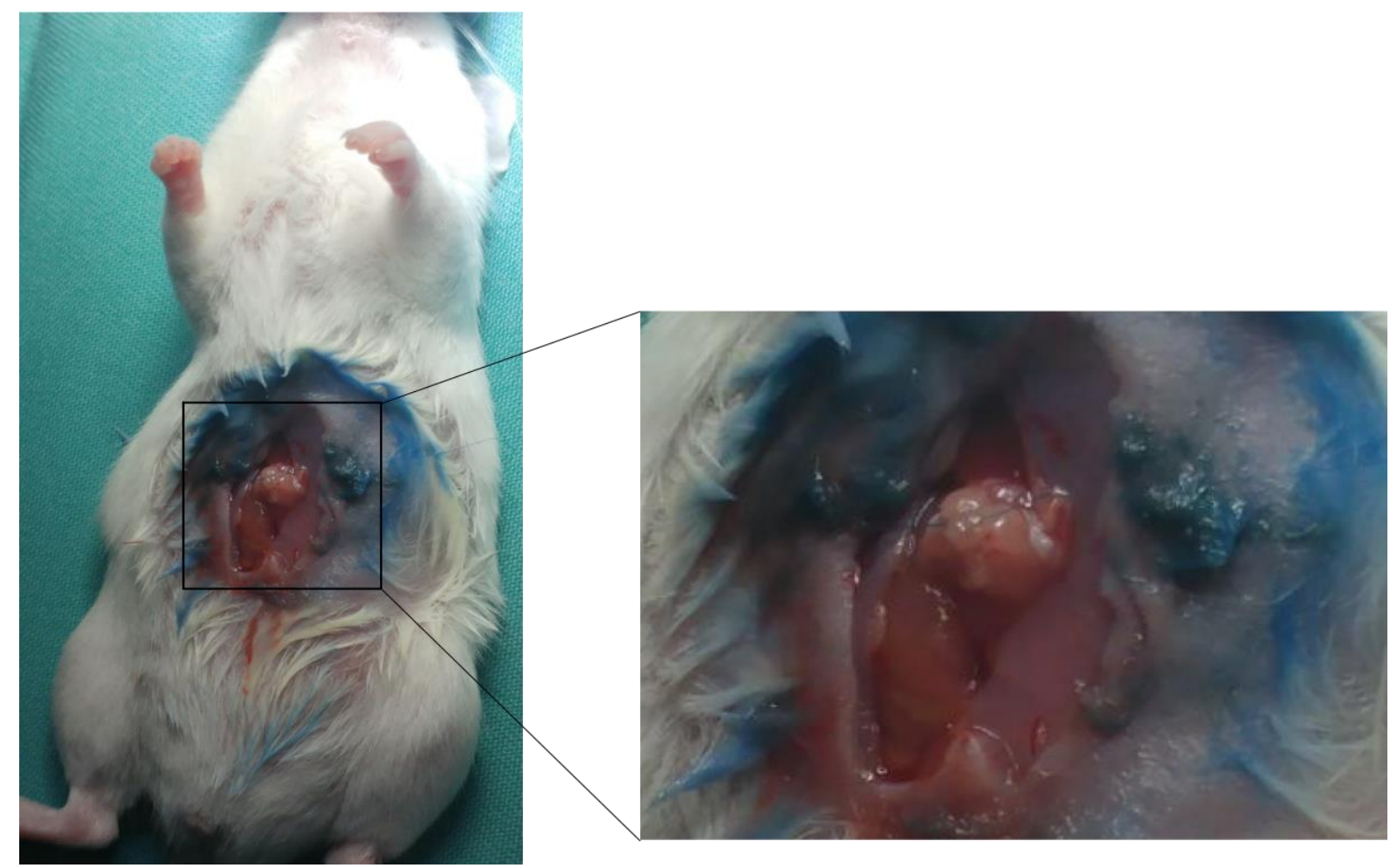

Figure 25. Surgical procedure for the removal of bioengineered ECM-hydrogels with HepG2 from the omentum of immunodeficient mice.

Mice hosting the ECM-hydrogels exhibited a normal behaviour during the course of the experiment and did not show any sign of local or systemic inflammation or infection.

At the end of this 3 weeks experiment, samples were retrieved from the omentum of the mice and the macroscopic analysis indicated no sign of redness or swelling in the implanted area, suggesting that inflammation did not occur during the course of the experiment.

Retrieved samples were then fixed in $4 \%$ formalin and analysed through Haematoxylin an Eosin staining (Figure 25) and immunohistochemistry staining (Figure 26) to evaluate if the HepG2 cells were still alive and engrafted into the implanted ECMhydrogel and to have a microscopic indication of signs of inflammation response.

Haematoxylin an Eosin staining (Figure 25 a-c) showed that ECM-hydrogels were attached to the liver of the mice and were fully repopulated with cells with a formation of nodules. Moreover, images did not show important signs of inflammation inside or 
around the implanted hydrogel, as confirmed by the absence of giant cell granulomas or formation of a thick fibrotic connective capsule.

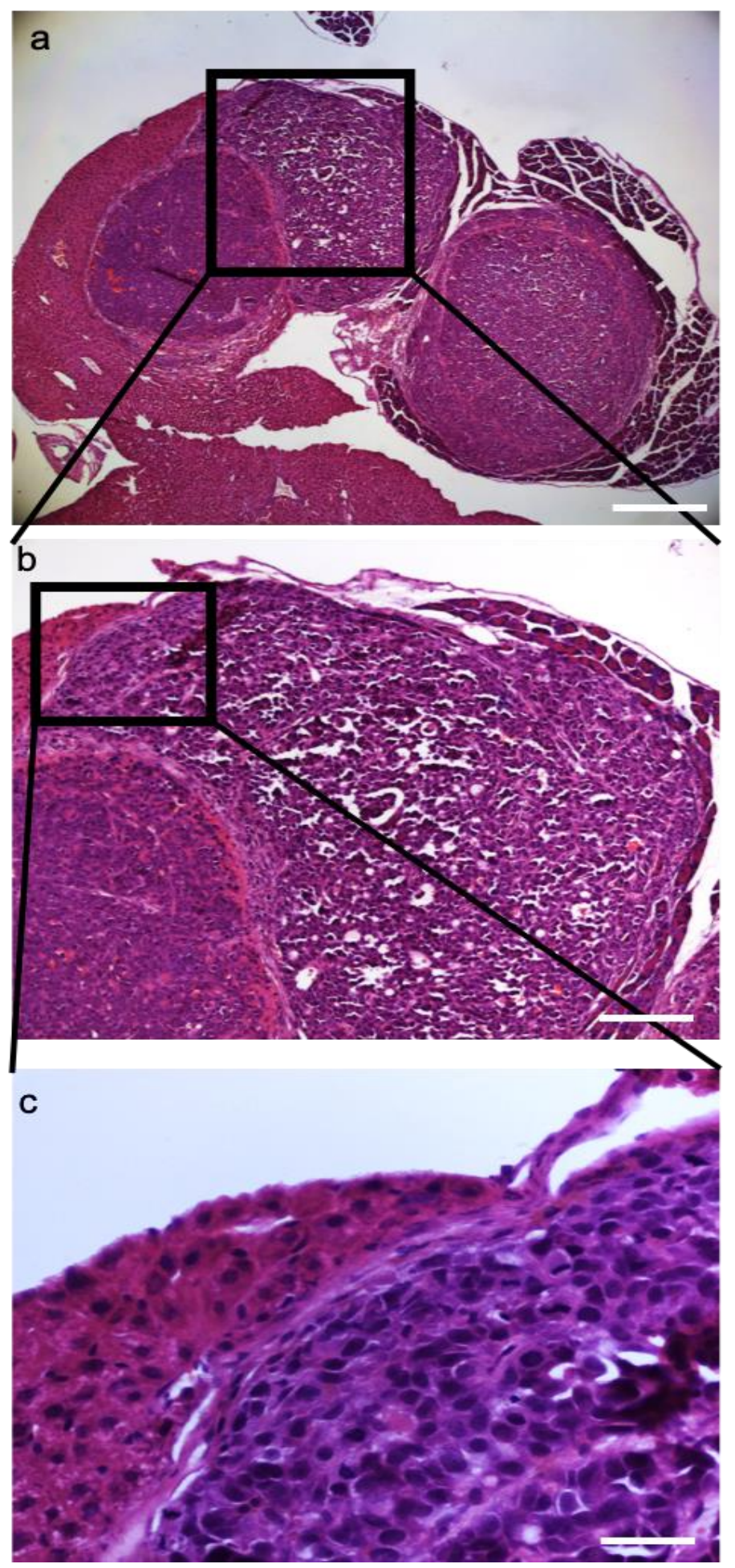

Figure 26. Haematoxylin an Eosin staining of bioengineered ECM-hydrogels with HepG2 implanted for three weeks into the omentum of immunodeficient mice. Scale bar (a) $500 \mu \mathrm{m}$, (b) $200 \mu \mathrm{m}$ and (c) $50 \mu \mathrm{m}$. 
Immunohistochemistry staining for human Ku80 was performed to evaluate if the implanted ECM-hydrogels were repopulated by the originally seeded human HepG2 cells (HepG2) or were repopulated with host cells. Resultant immunohistochemistry image (Figure 26) confirmed that the nodules were composed of HepG2 cells.

This result showed that HepG2 cells were alive and engrafted into the ECM-hydrogels after 3 weeks in the omentum of the mice, confirming the feasibility of using ECMhydrogels for in vivo applications, such as cell transplantation for the treatment of metabolic liver diseases.

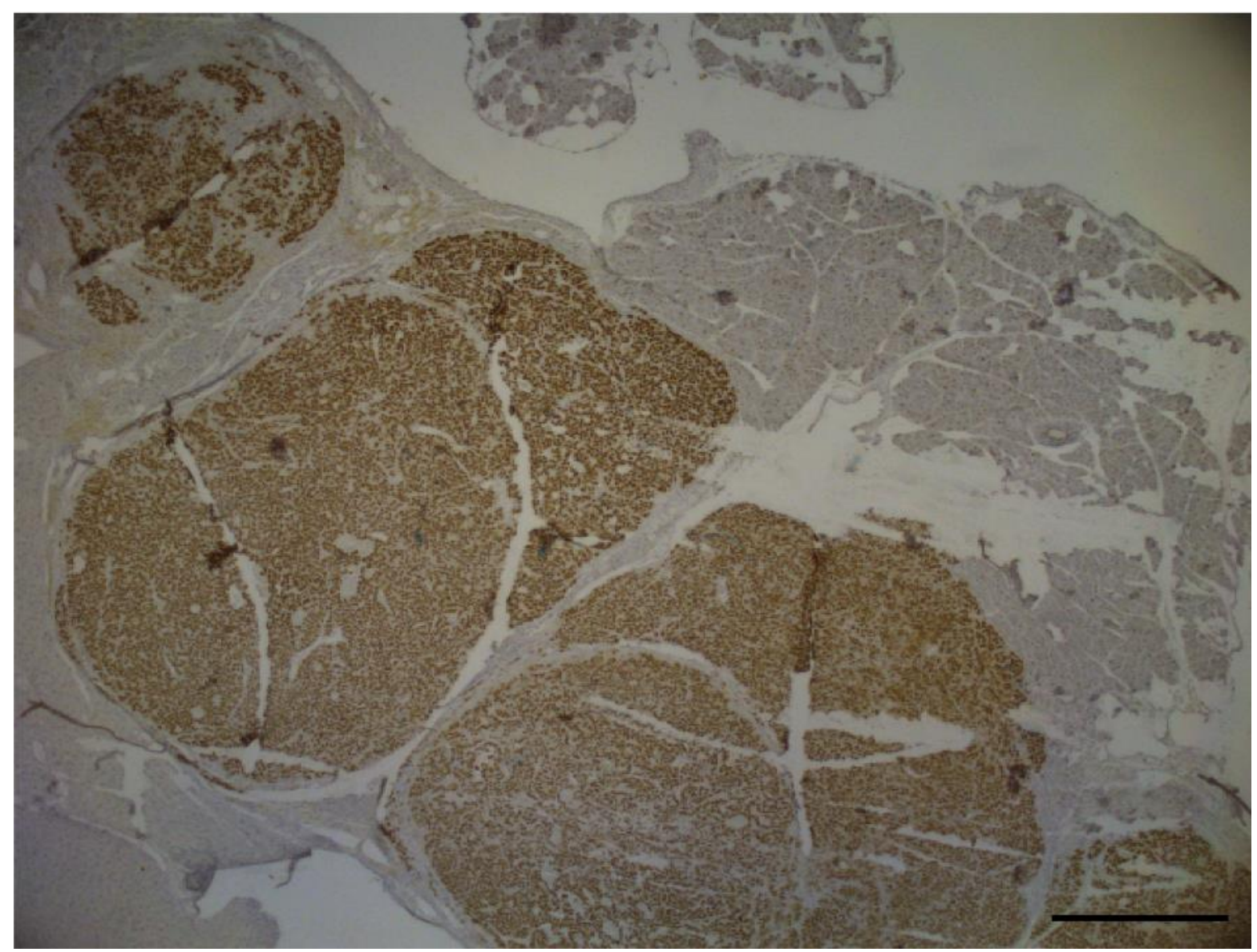

Figure 27. Immunohistochemistry staining for human Ku80. Resulting images confirmed that HepG2 were engrafted into the implanted ECM-hydrogels. Scale bar $500 \mu \mathrm{m}$. Immunohistochemistry staining was performed by Dr Mustapha Najimi, Université Catholique de Louvain, Laboratory of Paediatric Hepatology \& Cell Therapy, Institute of Experimental and Clinical Research, Belgium. 


\subsubsection{Evaluation of Human Induced Pluripotent Stem Cells (IPSC) differentiation on ECM-hydrogels}

The aim of this experiment was to evaluate the differentiation of human iPSC towards hepatocyte-like cells in 3D human liver ECM hydrogel. As previously described in the methods and materials (paragraph 2.3.9), IPSC differentiated for 12 days on 2D plastic were cultured for 18 days in 3D ECM-hydrogels, IPSC differentiated for 23 days on 2D plastic were cultured for 7 days in 3D ECM-hydrogels, while IPSC differentiated for 30 days on 2D plastic were cultured for 7 days in 3D ECM-hydrogels.

Differentiation towards hepatocyte-like cells of IPSCs cultured on ECM-hydrogels was evaluated through albumin quantification analysis, gene expression and immunohistochemistry.

Albumin secretion analysis (Figure 27) from iPSC cultured on ECM-hydrogels showed a time dependent statistically significant increase of secreted albumin from iPSC differentiated in 2D for 30 days and cultured for 3 and 7 days into 3D ECM-hydrogels. Similar result was obtained by IPSC seeded after 23 days of 2D differentiation into 3D ECM-hydrogels for both 3 and 7 days. The best result in terms of albumin secretion was obtained by IPSC differentiated for only 12 days in 2D and cultured for 18 days on ECM-hydrogels. 


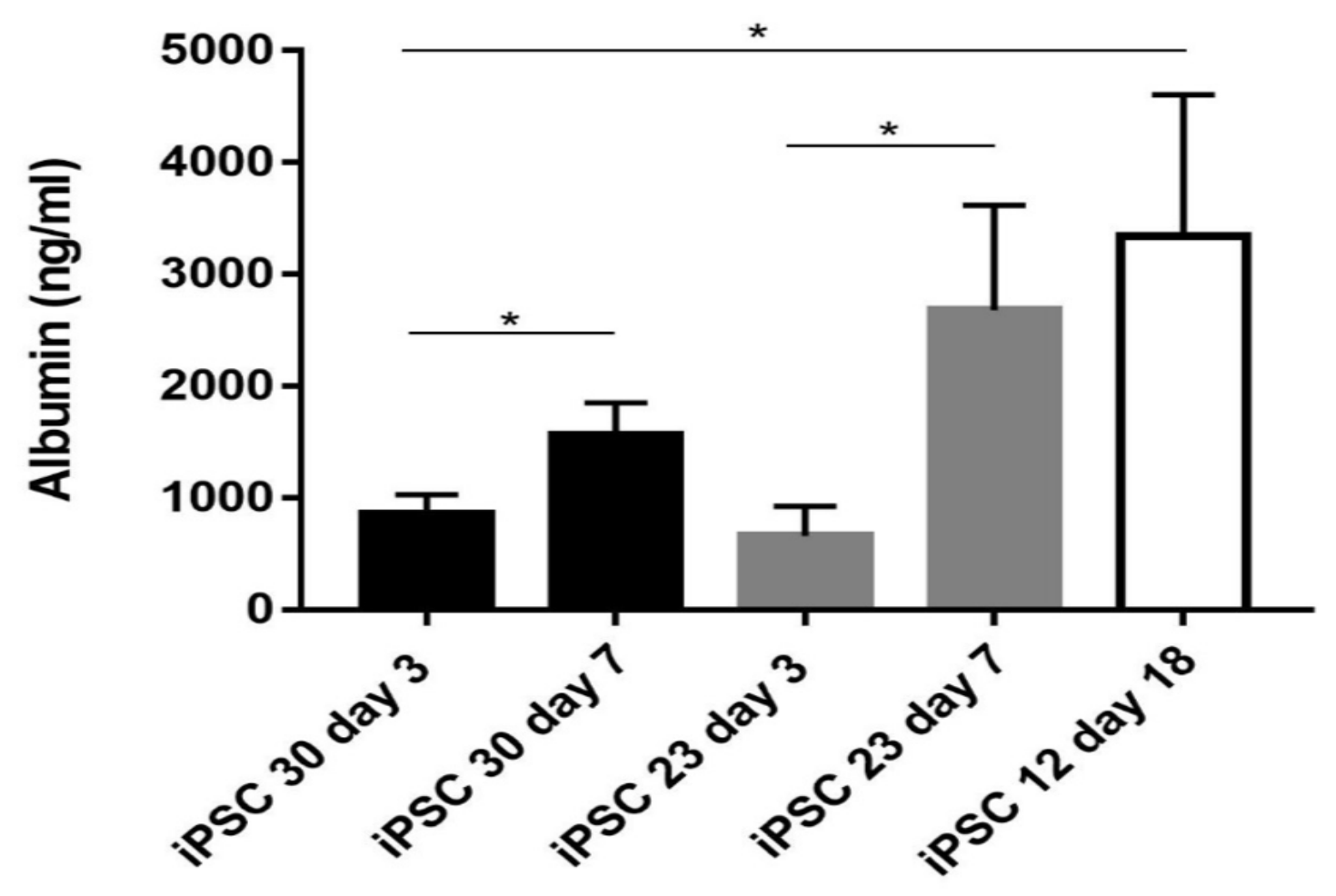

Figure 28. Albumin secretion of IPSC previously differentiated for 30 days on 2D fibronectin coated plastic (IPSC 30) was analysed after 3 and 7 days of culture in ECM-hydrogels (black bars). Albumin secretion of IPSC previously differentiated for 23 days on 2D fibronectin coated plastic (IPSC 23) was analysed after 3 and 7 days of culture in ECM-hydrogels (grey bars). IPSC previously differentiated for 12 days on 2D fibronectin coated plastic (IPSC 12) was analysed after 18 days of culture in ECM-hydrogels (white bar). ${ }^{*} p<0.05$. $n=4$ per condition.

Considering that IPSC differentiated for 7 and 18 days in ECM-hydrogels achieved the best results in terms of albumin secretion, these two conditions were further analysed through gene expression analysis in order to investigate the gene levels of important hepatocyte markers, such as HNF4A, UGT1A1, NR1H4, OTC, Albumin and AFP ( $n=4$ per condition).

Gene expression analysis (Figure 28) showed that HNF4A and UGT1A1 gene levels $(\mathrm{c}, \mathrm{f})$ were statistically significant up-regulated in IPSC 12 (cells differentiated for 12 days on 2D plastic and 18 days in 3D ECM-hydrogels) compare to IPSC 23 (cells differentiated for 23 days on 2D plastic and 7 days in 3D ECM-hydrogels). Albumin and NR1H4 gene levels (a,d) were up-regulated in IPSC 12 compare to IPSC 23 without statistically significant difference, while OTC gene level (e) was downregulated in IPSC 12 compared to IPSC 23 without statistically significant difference. 
AFP gene level (b) was statistically significant down-regulated in IPSC 12 compared to IPSC 23.

These results confirmed that the efficiency of IPSC differentiation towards hepatocytelike cells was better if these cells were cultured on ECM-hydrogels from an earlier stage (hepatoblast stage).

a

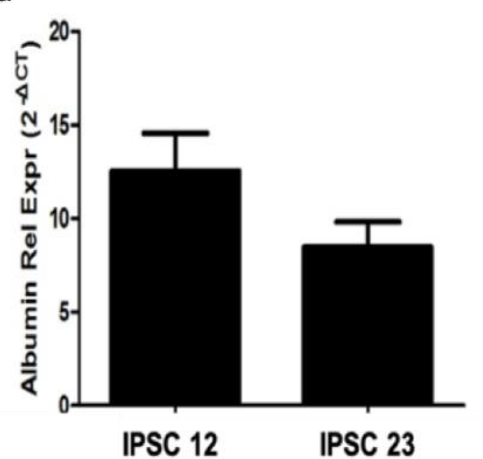

d

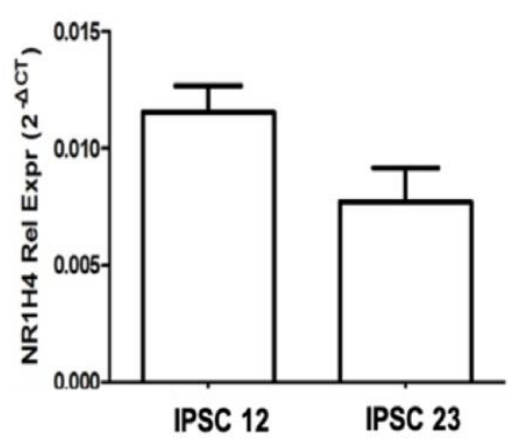

b

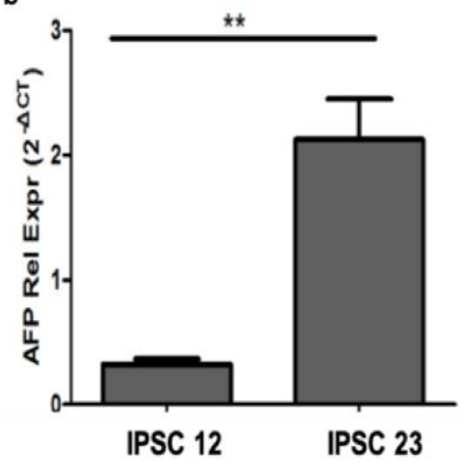

e

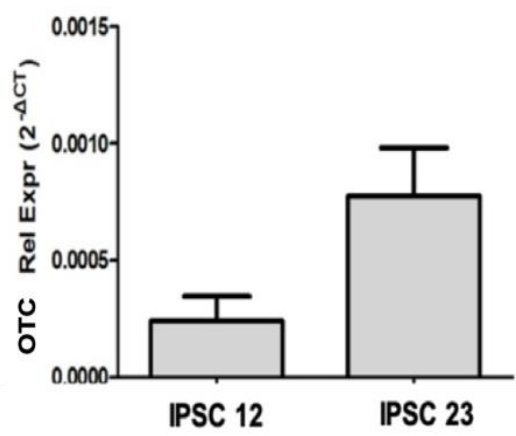

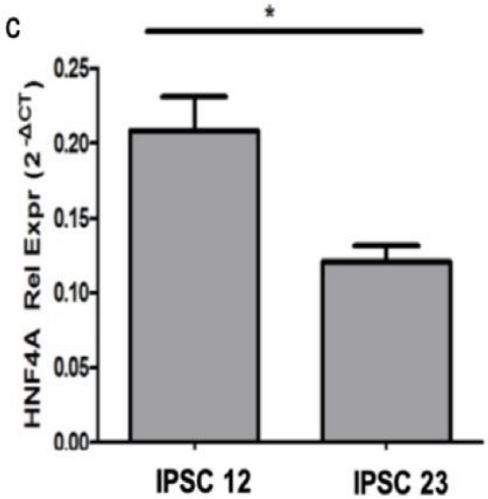

f

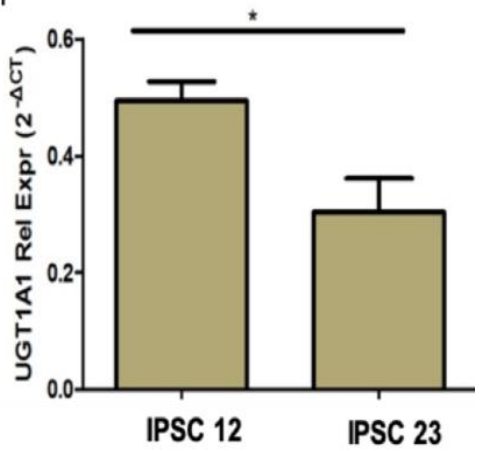

Figure 29. Gene expression analysis of IPSC cultured for 18 days (IPSC 12) and 7 days (IPSC 23) on $3 D$ ECM-hydrogels. ${ }^{*} p<0.05,{ }^{* *} p<0.01$. $n=4$ per condition.

Immunofluorescent images (Figure 29) captured for IPSC cultured on ECM-hydrogels for 18 days and on plastic as control, confirmed that IPSC cultured on ECM- hydrogels expressed hepatic differentiation markers including HNF4A, A1AT and Albumin. 

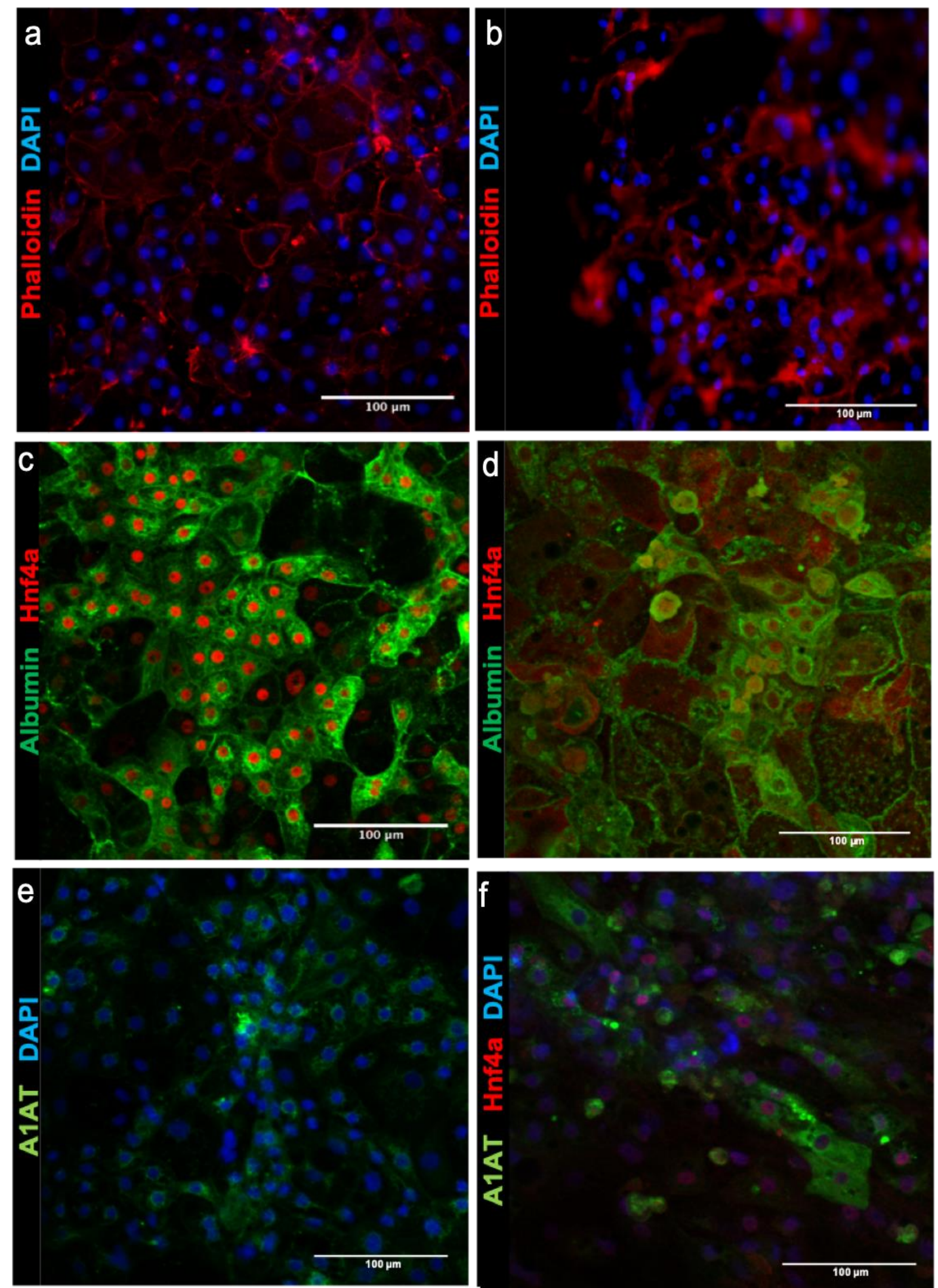

Figure 30. Immunofluorescent images of IPSC cultured for 18 days on ECM-hydrogels $(b, d, f)$ and on plastic $(\mathrm{a}, \mathrm{c}, \mathrm{e})$ as control. Resulting pictures confirmed that IPSC cultured on ECMhydrogels express hepatic differentiation markers including HNF4A, A1AT and Albumin. Scale bar (a-f) $100 \mu \mathrm{m}$. 
To conclude, this part of the study demonstrated that hepatocyte-like cells derived from iPSC technology are able to differentiate and mature in 3D ECM-hydrogels. The differentiation efficiency can be improved if hepatocyte-like cells are cultured from an earlier stage (i.e. hepatoblasts) in 3D ECM-hydrogels. This is a key advancement in the development of personalized 3D technologies for the study of liver diseases and for the understanding of liver development. 


\subsection{Discussion}

\subsubsection{Advantages of using human livers unsuitable for transplantation}

Decellularised ECM powder obtained from different organs and species have previously been utilized to develop ECM-based scaffolds. Examples include the porcine small intestinal submucosa, porcine liver, porcine urinary bladder and porcine adipose tissue. However, besides species specific, the physical and biochemical composition of the ECM is also tissue-specific. For example, $90 \%$ of the porcine small intestinal submucosa ECM is composed by collagen (predominantly type I), with minor amounts of type III, IV, V and VI collagens, glycosaminoglycans (GAGs), fibronectin and laminin as well as growth factors. Porcine urinary bladder matrix is similar in composition, but with greater amounts of type III collagen and type VII collagen originating from the endothelial basement membrane [222]. Furthermore, despite the fact that basic components of the ECM showed a considerable degree of conservation among species, the porcine liver ECM is characterised by well-defined lobules delineated by connective tissue, which are not present in healthy human livers but detected in fibrotic human livers [213]. Therefore, to recreate the physiological microenvironment for the in vitro culture of the human hepatic cells one would favour human liver as most suitable source of ECM.

\subsubsection{Solubilisation and gelation of human liver ECM}

The experiments and results described in this thesis demonstrated the possibility of developing a novel protocol for the preparation of human liver ECM-hydrogels starting from human livers unsuitable for transplantation.

All published protocols so far used for the development of ECM-based hydrogels employ pepsin for the digestion of the ECM powder. As described in this Chapter, the first two protocols (Protocol 1 and 2) used to solubilise the human liver ECM powder were based on the use of pepsin. Particularly in the case of the second protocol tested (Protocol 2), pepsin was used in a high concentration to digest ECM powder. Indeed, 
the ratio between human liver ECM components and pepsin in the solution was 5 to 1. This xenogeneic enzyme cannot be removed from the ECM solution, therefore ECM-hydrogels prepared with above mentioned digestion step, contain a high quantity of this enzyme and thus present a disadvantage for our proposed applications such as in vivo implantation/transplantation and in vitro drug screening model. Indeed, the homology between human and porcine pepsin is around 82\% [223] and therefore the presence of this xenogeneic enzyme into the ECM-hydrogels might cause activation of the host immune system when implanted in humans. Thus, the main advantage of Protocol 3 and an important step for future applications is that the obtained human liver ECM solution does not contain pepsin.

Following the novel protocol described in this thesis, human liver ECM solution was prepared with acetic acid and an additional sonication step to completely solubilise the ECM powder.

For all three 3 tested solubilisation protocols, the final ECM concentration was calculated according to the initial ECM powder weighed and assuming that there was not any lost of ECM components during the steps. However, protocol 3 is characterised by several steps and, particularly during the ultracentrifugation step, part of the ECM components might be lost. A possible way to better measure the final ECM concentration is to resuspend in deionised water the pellet obtained after ultracentrifugation step, freeze-dry the resultant solution and then weigh the powder. Moreover, the different ECM components resuspended in deionise water can be quantified by performing analysis of total proteins (Bicinchoninic acid assay), analysis of hydroxyproline content for collagens, analysis of elastin content and glycosaminoglycans (GAGs) content.

Furthermore, one important difference that was noticed is that the human liver ECM solution did not show self-assembling properties previously demonstrated by others xenogeneic organs ECM solutions, such as porcine liver ECM solution [186], porcine small intestine mucosa ECM solution [184] and porcine urinary bladder ECM solution [113] further indicating species specific characteristics. This observation is consistent with the study published by Loneker et al in 2016, where is shown that porcine, rat and canine liver ECM solutions were able to turn into gel when temperature increased to $37^{\circ} \mathrm{C}$, while the human liver ECM solution, at the same concentration of $8 \mathrm{mg} / \mathrm{ml}$, did 
not show any increase of storage modulus after being at $37^{\circ} \mathrm{C}$ for more than 30 minutes [185].

As shown in this Chapter, to overcome this limitation of the human liver, gelling agents such as agarose and agar were employed to gel the human liver ECM solution.

\subsubsection{Characterisation of developed ECM-hydrogels}

The resultant human liver ECM-hydrogels were characterized by the retention of most abundant ECM proteins, such as collagen I, collagen III, laminin, fibronectin and elastin as shown by immunohistochemistry. Presence of these ECM proteins is crucial to provide a more physiological microenvironment for the hepatic cells and allow the cells to behave more in vivo like. All developed ECM-hydrogels (containing agar $0.125 \%-1 \%$ and agarose $0.25 \%-1 \%$ ) were composed of the same concentration of ECM components $(4 \mathrm{mg} / \mathrm{ml})$ but different volumes of agar/agarose solutions and $1 \mathrm{x}$ PBS solution. For example, in the case of ECM-hydrogels containing agarose $0.25 \%$, the ECM solution was combined with more $1 \mathrm{X}$ PBS solution and less agarose solution than ECM-hydrogels containing agarose $0.5 \%$ or $1 \%$. For this reason, considering that all different ECM-hydrogels contained the same volume of ECM solution, in this Chapter were shown only the immunohistochemistry pictures of the samples containing agarose $0.25 \% \mathrm{w} / \mathrm{v}$.

A PAGE gel stained with Coomassie Blue and a western blot of the different collagens, laminin, fibronectin would have been important to further confirm the presence of these ECM proteins in the gels. Furthermore, would have been useful to perform a western blot of these ECM components pre- and post- ECM-hydrogels sterilisation to exclude the possibility that peracetic acid caused denaturation of a good portion of the proteins present.

The ultrastructure of developed ECM-hydrogels was investigated by Scanning Electron Microscopy (SEM). Resultant images showed the presence of and collagenic structures and an adequate porosity. In comparison to the porcine liver ECMhydrogels [224], the ultrastructure of human liver ECM-hydrogels seemed to be characterised by thicker fibres, suggesting a more stable and stiffer scaffold, and 
higher porosity. This is an advantage because porosity is a feature needed to allow diffusion of oxygen and nutrients for hepatic cells during their in vitro culture.

Rheological characterisation of ECM-hydrogels confirmed the very stable structure and this due to the usage of covalent cross-linkers and mechanical properties which were not affected by changes in temperatures. This is an important feature for the application of the ECM gels in in vivo applications such as injection/transplantation.

An important observation was that the stiffness of the ECM-hydrogels containing $0.25 \%(\mathrm{v} / \mathrm{v})$ of agarose was $1.2 \mathrm{kPa}$ at $37^{\circ} \mathrm{C}$, a value similar to that of normal human livers [225]. This indicates that ECM-hydrogels are able to mimic mechanical properties of a healthy human liver. This further enforces the usage of 3D ECM human liver hydrogels in in vitro experiments over 2D or Matrigel-based cultures. Indeed, the mechanical signals of the cell culture environment are known to be crucial in determining cell phenotype and activity [226]. Plastic surfaces are still used for the majority of cell culture, but the stiffness of this standard culture platform is around $10.000 \mathrm{kPa}$ [227] and cells failed to reproduce the in vivo like behaviour. Conversely, the human liver ECM-hydrogels are characterised by a stiffness similar to that of healthy human livers, a crucial feature to maintain the original cell phenotype and activation status.

The viscoelastic profile of ECM-hydrogels containing $0.25 \%(\mathrm{v} / \mathrm{v})$ of agarose was further investigated by performing a frequency sweep test between $0.019 \mathrm{~Hz}(0.12$ $\mathrm{rad} / \mathrm{s})$ and $38.9 \mathrm{~Hz}(244.5 \mathrm{rad} / \mathrm{s})$. The viscosity of the ECM hydrogel, in the frequency range of $0.01 \mathrm{~Hz}$ and $15 \mathrm{~Hz}$, is similar to that of the collagen type I injectable gel and porcine urinary bladder ECM gel [113], confirming that this novel 3D platform can be used for cell delivery in clinic, avoiding risks correlated to surgical invasive procedures.

\subsubsection{Sterilisation of ECM-hydrogels}

Sterilization of biological samples for tissue engineering and regenerative medicine applications is a necessary step to eliminate bacteria, virus, fungal and spores. The

most common sterilisation techniques include gamma irradiation, ultraviolet irradiation, ethylene oxide, peracetic acid, ethanol $70 \%$ and glutaraldehyde [228]. All 
these methods negatively modify natural properties of biomaterials, including biochemical composition, 3D structure and physical integrity, thus affecting cell adhesion, proliferation and cellular behaviour [229].

In 2014 Matuska and McFetridgea investigated the effects of three common sterilisation techniques on decellularized porcine temporomandibular joint samples: gamma irradiation, ethylene oxide, and peracetic acid/ethanol (peracetic acid $0.2 \%$ and ethanol $4 \%$ in deionised water). All sterilization methods negatively affected 3D ultrastructure, biochemical composition and physical stability. SEM images showed higher modifications of the microstructure when samples were gamma irradiated or exposed to ethylene oxide compared to peracetic acid/ethanol sterilisation method. Furthermore, adhesion and proliferation of Human umbilical cord Wharton's Jelly matrix cells (hWJMC) after 1 and 4 days was shown to be superior in peracetic acid/ethanol sterilized scaffolds compared to gamma irradiated or ethylene oxide treatments [228].

For this reason, peracetic acid/ethanol was chosen as a sterilization method for the described ECM hydrogels. Furthermore, knowing that the oxidative and acidic environment created by peracetic acid treatment causes protein denaturation, the concentration of this reagent was reduced to $0.1 \%$. The reduction of acid concentration did not affect the effectiveness of the sterilisation treatment.

\subsubsection{In vitro biocompatibility of ECM-hydrogels}

Human primary hepatocytes represent a golden standard for in vitro liver research and in vivo applications for the treatment of liver diseases [230]. Due to their low availability and high costs, human hepatoblastoma cell line HepG2 were employed in this study as a tool for human hepatocytes, since they present comparable morphological and biochemical characteristics [231].

The in vitro biocompatibility of human liver ECM-hydrogels, containing different concentrations $(\mathrm{v} / \mathrm{v})$ of agar or agarose, was evaluated by seeding HepG2 cells. The HepG2 cells engrafted and migrated into the different 3D scaffolds while maintaining the metabolic phenotype, except for the hydrogels containing agar $0.25 \%(\mathrm{v} / \mathrm{v})$. One 
possible explanation is that the ECM hydrogel containing agar $0.25 \%(\mathrm{v} / \mathrm{v})$ was too soft for the HepG2 cells as cells migrated through the gel towards the plastic of the well plate.

The importance of ECM proteins for cell attachment, viability and functionality was further tested by culturing HepG2 cells up to 10 days. Attachment and viability of seeded HepG2 cells was investigated by histological and immunofluorescence staining. Haematoxylin and eosin staining of ECM hydrogel in agarose 0,25\% confirmed that HepG2 attached and migrated into the scaffold. No cells were found in hydrogels composed only by agarose $0.25 \%$ confirming the inert features of agarose $[98,232]$. Cell morphology analysis with fluorescent DAPI and fluorescent phalloidin confirmed the retention of the natural hexagonal shape of HepG2 cells in the gels which is lost upon 2D culturing [233]. Human Albumin ELISA data demonstrated a time dependent increase of secreted human albumin from ECM-hydrogels while in contrast albumin was not detectable in hydrogels made of agarose only. Further indicating a favourable role of using human liver ECM gel for culturing HepG2 cells. Furthermore, these data demonstrate how the human liver ECM microenvironment positively influences cell attachment, viability and functionality.

Additionally, ECM-hydrogels with agarose $0.25 \%(\mathrm{v} / \mathrm{v})$ were able to maintain the HepG2 cells alive and functional for up to 19 days. This is in strong contrast with previously published work of HepG2 cells cultured in 2D plastic, where it was demonstrated that these cells lose their specific hepatic function after 10 days in culture [63]. The Alamar Blue viability assay showed a steady state in cell number from day 7 to day 13 while from day 13 to day 19 the cell number significantly increased. This was further corroborated by performing a Human Albumin ELISA. These data confirmed that during the first days in culture a reduction in albumin secretion was observed from day 7 to day 13 which was followed by a statistically significant increase from day 13 to 19 days. Similar results were observed when primary human hepatocytes were cultured in human 3D liver scaffolds where lactate dehydrogenase (LDH) was strongly increased during the first days of repopulation to decrease and being not detectable at day 10 , and at the same time albumin and factor IX secretion was significant upregulated. This indicates that the repopulation of cells, or contact with human ECM, requires/induces a certain cellular adaption [154]. 
Albumin secretion and gene expression of HepG2 cells cultured in ECM-hydrogels were compared to those of cells cultured in acellular liver cubes employed as an internal 3D platform control. Haematoxylin and eosin staining of ECM-hydrogel in agarose $0,25 \%(\mathrm{v} / \mathrm{v})$ and $3 \mathrm{D}$ liver cubes after 13 days showed different organization/localization of these cells. Indeed, HepG2 cells cultured in the ECMhydrogels were organised in spheroids within the gel, whereas HepG2 cells repopulating the 3D liver cube did not migrate within the ECM. The higher cell-to-cell contact, intrinsic of cluster organisation, might explain the higher metabolic profile of HepG2 cells cultured in ECM-hydrogels as was shown for HepG2 cells in spheroids [62]. A critical problem of the ECM-hydrogels is the difficulty to normalise results according to the concentration of DNA or cell number. This is due the presence of Agarose that interferes with the extraction of cells and mRNA. Indeed, from the histological images HepG2 cell number seems higher in the ECM-hydrogels compared to acellular liver cubes. mRNA extraction result was completely the opposite and not reliable since $314.2 \pm 41.4 \mathrm{ng} / \mathrm{ul}$ of mRNA were obtained from acellular liver cubes and $25.9 \pm 3.5 \mathrm{ng} / \mathrm{ul}$ of mRNA were obtain from ECM-hydrogels. For this reason, albumin ELISA results were not normalized to avoid false results. Nevertheless, gene expression results using GAPDH as housekeeping gene, confirmed that metabolic activity was higher in HepG2 cultured in ECM-hydrogels compared to acellular liver cubes.

Although in ECM-hydrogels the native ultrastructure of healthy livers is not preserved as in the acellular liver cubes, the main advantage of this novel 3D platform is the possibility to produce gels at large scale within a reasonable time.

\subsubsection{In vivo biocompatibility of ECM-hydrogels}

ECM-hydrogels bioengineered with HepG2 cells were implanted into the omentum of immunodeficient mice in order to evaluate their in vivo biocompatibility and ability to maintain implanted human cells alive and engrafted post implantation. Histological and immunohistochemistry analysis of samples implanted for 3 weeks into the omentum of mice showed that ECM-hydrogels did not cause inflammation in the abdominal area and important HepG2 cells were alive and still engrafted into the implanted area. On 
the contrary, the current clinical use of hepatocyte transplantation for the treatment of liver disease showed limited patient benefits due to poor cell engraftment and a time limited cell survival after transplantation [66]. For this reason, ECM-hydrogel could be tested for hepatocyte transplantation to enhance cell engraftment and survival after the surgical procedure.

\subsubsection{IPSC differentiation towards hepatocyte like cells}

The development of induced pluripotent stem cells (IPSCs) holds great promise for the realization of personalised regenerative medicine and in vitro disease models [234, $235](82,83)$.

IPSCs cells can be derived directly from the patient's own fibroblasts, differentiated into a variety of adult somatic cells, including hepatocytes [236], choanocytes [237], pancreatic beta-cells [238], cardiomyocytes [239], neural cells [240] and used for autologous transplantation in order to restore the functionality of the damaged organs without the need of an immunosuppressive therapy [241]. Furthermore, IPSCs cells can be used as in vitro models to study the mechanisms underlying human diseases, to study tissues and organs regeneration and for the screening of new drugs [242]. Important, IPSC differentiated in hepatocyte-like cells can also overcome the problem correlated to the limited source of available hepatocytes for both in vitro and in vivo applications [243].

This study demonstrated that hepatocyte-like cells derived from iPSCs are able to differentiate and mature in 3D ECM-hydrogels. Albumin secretion analysis and gene expression showed that the differentiation efficiency was improved when iPSCs were cultured from an earlier stage (i.e. hepatoblasts) in 3D ECM-hydrogels, confirming that ECM components play an important role in promoting the differentiation of IPSCs towards hepatocytes-like cells. For this reason, ECM-hydrogels bioengineered with IPSCs cultured from an early stage have the potential to be used as in vitro drug screening model as well as for the in vivo cell transplantation for the treatment of metabolic liver disorders. 
Similar to previous experiments described in this Chapter, normalisation of albumin ELISA results was not performed considering the low efficiency of cells and mRNA extraction from ECM-hydrogels. Furthermore, Primary human hepatocytes should have been used as a control for both albumin secretion and gene expression to better evaluate the differentiation of IPSCs cultured in ECM-hydrogels. In addition, IPSCs differentiated for 30 days in $2 \mathrm{D}$ should have been compared to bioengineered ECMhydrogels to evaluate if cells cultured in 3D better differentiated towards hepatocyteslike cells.

Altogether, the current study demonstrated the feasibility of employing human liver ECM-hydrogels for in vitro drug discovery and drug toxicity testing as well as for in vivo application. The main disadvantage of this human liver ECM-based hydrogel is the re-seeding technique because cells are added on top of the hydrogels and this causes variability due to a different cell attachment. In addition, the high number of cells needed limits the use of ECM-hydrogels for the high throughput screening (HTS) of new drugs for the treatment of liver disease. 


\section{Chapter 3: 3D Bio-printing of Human Hepatic tissue using Human Liver Extracellular Matrix as Tissue-Specific Bio-ink}

\subsection{Introduction}

This Chapter addresses the bio-printing of human liver ECM solution in combination with a biocompatible bio-ink (nanocellulose based bio-ink, provided by CELLINK AB) in order to bio-print human tissue specific samples for subsequent use in in vitro culture models and research applications in drug screening and development. Data reported in this Chapter have been incorporated into a patent (International patent application No. PTC/EP2018/086632).

\subsubsection{History of 3D printing}

In the last decades, printing technology has evolved from two-dimensional (2D) printing to an additive process of multiple layers of material to form 3D constructs [244].

In 1986 Charles W Hull, under the name of "sterolithography", described for the first time the 3D printing method, in which thin layers of a plastic material were overlaid one on top of the other and cross-linked with ultraviolet light to obtain solid 3D objects [245]. The 3D printing process was then later applied in many areas, including engineering, manufacturing, and medicine [77].

Recent advances in the 3D printing technology, with the possibility of printing cells in combination with biocompatible materials and the improved precision in the spatial deposition of the bio-inks, have allowed this technique to be applied in the field of tissue engineering and regenerative medicine to produce complex 3D functional samples able to recapitulate the microenvironment of native tissues and organs [246]. 


\subsubsection{Natural and synthetic bio-inks for 3D printing}

A crucial aspect for the bio fabrication of functional 3D samples is the choice of the bio-ink [247]. Bio-inks should exhibit several critical properties, including printability, mechanical properties, biodegradation, biological biocompatibility, cytocompatibility, and bioactivity [248]. Another key aspect is the permeability through the bio-printed constructs of oxygen gas, nutrients and metabolic wastes [78].

Additionally, a specific bio-ink should be selected according to the properties and characteristics of the desired tissue or organ in order to mimic the native microenvironment and allow the specific cells to behave in-vivo like [249].

In this context, several natural and synthetic biomaterials, such as agarose, collagen, alginate, cellulose, hyaluronic acid and polyethylene glycol (PEG) have been employed as bio-inks for 3D printing [78]. Other synthetic biomaterials, such as polycaprolactone (PCL) and polylactic acid (PLA) are extensively used in 3D printing for the fabrication of 3D scaffolds for bone [250] and tubular organs bioengineering [251]. Since PLC and PLA could only be printed at elevated temperatures and at high extrusion pressure, they are not ideal bio-inks for live cell printing [252, 253].

Agarose, a natural polysaccharide derived from seaweeds, is one of the most employed biopolymers for biomedical applications due to its excellent mechanical and biocompatibility properties [254]. However, cell culture in agarose constructs is characterised by low cell adhesion and limited cell proliferation rate, suggesting that agarose is a poor material for cell culture [255]. To take advantage of the mechanical properties of agarose and overcome the cell culture deficits, agarose was bio-printed in combination with more bioactive polymers such as collagen or fibrinogen to obtain 3D constructs able to support in vitro cell culture [256]. In another work, agarose was mixed with collagen and sodium alginate and bio-printed with chondrocytes for cartilage regeneration [257].

Alginate is a natural polysaccharide extracted from brown algae [258], extensively used in bio-printing processes due its low price, biocompatibility, ease of crosslinking and excellent gel formation to fabricate 3D structures [259]. For example, sodium alginate bio-ink, containing mouse fibroblast cells, was used to bio-print 3D biological vascular trees using calcium chloride as cross-linking agent [260]. 
To improve the limited cell adhesion and cell proliferation properties of alginate [261], the biomaterial was bio-printed in combination with collagen to develop 3D constructs with chondrocytes. The authors showed that the presence of collagen avoided the dedifferentiation of bio-printed cells and increased cell attachment and proliferation [257].

Collagen type $\mathrm{I}$ is one of the most abundant components of ECM and is formed by a triple helical structure [262]. Collagen type I is one of the most used bio-ink for 3D cell printing because of its excellent cell attachment and growth properties [263]. The biggest limitation of using collagen for the bio-printing of 3D constructs is its slow gelation rate at $37^{\circ} \mathrm{C}$ that affects the structural resolution and shape definition of the bio-printed constructs. Indeed, complete gelation requires up to 60 minutes at $37^{\circ} \mathrm{C}$ [264]. Another disadvantage when using collagen as biomaterial for 3D printing is related to its very low viscosity, thus cells are not homogeneously distributed into the collagen bio-ink as gravity pulls down the cells before gelation can take place [78]. To overcome these low mechanical properties and to increase the printability properties, collagen has been mixed with natural or synthetic supporting biomaterial such as alginate [257] or pluronic [265].

Hyaluronic acid (HA) is another ECM component abundantly present in cartilages and connective tissues [266]. This biomaterial, similar to collagen, is characterised by poor mechanical properties, slow gelation time and rapid degradation [267]. To enhance the bio-printability properties of hyaluronic acid, this polymer was chemically modified with methacrylate, obtaining a suitable bio-ink cross-linked by a photo-crosslinking mechanism [268].

Cellulose is a linear polysaccharide obtained from plants or bacteria [269]. Cellulosebased bio-inks, in combination with different biomaterial such as alginate, have been widely used for cartilage tissue engineering [270, 271].

Synthetic polymers do not usually have the same capability of natural polymer to promote cellular adhesion, growth and functionality, but they are helpful biomaterial to enhance the printability properties of natural bio-inks [272]. Within the synthetic polymers, pluronic and polyethyleneglycol (PEG) are the most used polymers for 3D bio-printing [249]. 
Pluronic is a synthetic copolymer composed of a hydrophobic chain connected to hydrophilic groups. Mozetic et al. reported that 3D bio-printed constructs composed of pluronic and alginate enhanced myoblast cell viability and alignment compared to traditional 2D cell culture [273].

The most used PEG-based bio-inks for 3D printing, are PEG-diacrylate and methacrylate [274]. Since PEGs are hydrophilic and are not able to support the cell culture, they were mixed with other natural polymers, such as alginate or collagen, or functionalized with biochemical cues by incorporating RGD (Arg-Gly-Asp) peptides [248].

\subsubsection{Decellularized extracellular matrix as a new source of bio-inks}

Following the recent improvements in the decellularization protocols of different organs and tissues, the decellularized extracellular matrix ( $\mathrm{dECM}$ ) became an attractive candidate as source of bio-ink because of its capability to retain the intrinsic bioactive cues and the appropriate microenvironment of the native organs and tissue to promote cell engraftment, survival, and function [79, 275, 276].

Due to the low printability performance of the extracellular matrix solutions, a supporting polymer, such as polycaprolactone (PLC), can be added to the ECM solutions [277].

So far, few studies have described the employment and the consequent advantages of using decellularized extracellular matrix ( $\mathrm{dECM}$ ) as a bio-ink for $3 \mathrm{D}$ cell printing. The first scientific publication in the field of ECM bio-inks was published in 2014 and the authors showed the feasibility of using porcine decellularized adipose, cartilage and heart tissues supported by a polycaprolactone framework. The authors demonstrated that the bio-printed 3D constructs containing dECM were characterised by high cell viability and functionality [278]. In another study published in 2017 by Lee et al., porcine liver ECM was combined together with polycaprolactone (PCL) as bioink for 3D-printer and the authors showed an improvement in metabolic function of bio-printed tissue in the presence of extracellular matrix [80]. Decellularised extracellular matrix based bio-inks were also applied for corneal tissue engineering. 
The authors showed that human mesenchymal stem cells differentiated into corneal keratocytes only when using a cornea ECM based bio-ink [279]. Human stem cells were also bio-printed using a human skin ECM based bio-ink, and the authors showed that the resultant bio-printed skin patches, used in mice, enhanced wound healing, neovascularization, and re-epithelialization [280]. 


\subsection{Aim and Objectives}

\subsubsection{Aim}

To use human liver extracellular matrix solution as bio-ink to bio-print human tissue specific constructs that are able to support the in vitro culture of human hepatic cells.

\subsubsection{Objectives}

To achieve this aim the following objectives will be considered:

1. Investigate the printability of human liver ECM solution combined with Cellink bio-ink employed as supporting biomaterial for bio-printing.

2. Assessing the in vitro biocompatibility by bio-printing Human Hepatoblastoma cells (HepG2 cell line), immortalised Human Hepatic Stellate cells (LX2 cell line) and Primary Human Hepatocytes.

3. Comparing gene expression and functionality of bio-printed hepatic cells between $\mathrm{ECM}+$ Cellink bio-ink and Cellink bio-ink employed as a control. 


\subsection{Methods and Materials}

\subsubsection{Development of Human Liver ECM solution}

Human livers were decellularized following a novel protocol developed and published by Mazza et al. in 2015. Next, the human decellularized liver was lyophilized (VIRTIS Benchtop) overnight to obtain a dried tissue that was then milled (rotary knife milling machine, Wiley Mini-Mill) to create human liver ECM powder as detailed described in the previous Chapter. Briefly, this powder was suspended in $0.5 \mathrm{M}$ acetic acid (Fisher scientific) (250 ml for $1 \mathrm{~g}$ of ECM powder), without the implementation of pepsin, at 4 ${ }^{\circ} \mathrm{C}$ for 72 hours under magnetic stirring. Human liver ECM solution was then ultracentrifuged (Beckman Coulter, LE-80 K Ultracentrifuge) at $10.000 \mathrm{~g}$ at $4{ }^{\circ} \mathrm{C}$ for 30 minutes. The pellet of human liver ECM was resuspended in acetic acid 0.25M (166.6 $\mathrm{ml}$ for $1 \mathrm{~g}$ of ECM powder) at $4{ }^{\circ} \mathrm{C}$ for 24 hours followed by sonication (Bandelin Sonoplus) ( 5 cycles of 20 seconds at $50 \mathrm{~Hz}$ ). The pH of the resultant ECM solution was then neutralised with $10 \mathrm{~N}$ sodium hydroxide ( $30 \mu \mathrm{l}$ per each $\mathrm{ml}$ of ECM solution) and the osmotic pressure was adjusted with 10X PBS (1:10 volume of ECM solution). This human liver ECM solution, characterised by an ECM concentration of $5,3 \mathrm{mg} / \mathrm{ml}$, was sterilised overnight by gamma-irradiation ( $1782 \mathrm{~Gy}$, using CIS bio international IBL $437 \mathrm{C}$ irradiator) at the Institute of Child Health (ICH) facilities at UCL.

\subsubsection{Maintenance of hepatic cells in culture}

HepG2, LX2 and primary human hepatocytes were used in this study.

HepG2 cells were cultured in MEM Medium (Gibco Thermo Fisher Scientifics) supplemented with 10\% Foetal Bovine Serum (FBS; Gibco Thermo Fisher Scientific), 2mM L-glutamine (200mM Gibco Thermo Fisher Scientific), 1\% Sodium Pyruvate (100X Gibco Thermo Fisher Scientific), 1\% Non-Essential Amino Acids (100X Gibco Thermo Fisher Scientific) and 1\% 1X Antibiotic-Antimycotic (100X Gibco Thermo 
Fisher Scientific). HepG2 cells were cultured in a humidified incubator at $37{ }^{\circ} \mathrm{C}$ with $5 \% \mathrm{CO}_{2}$. Once cells reached $\sim 75 \%$ confluence, cells were trypsinised using $0.25 \%$ Trypsin-EDTA (Thermo Fisher Scientific) and passaged at a split ratio of 1:3.

The LX2 is a human hepatic stellate cell line generated by immortalisation with Simian Vacuolating Virus 40 transforming antigen and consequent propagation in low serum conditions (kindly provided by Prof Scot Friedman) [281]. LX2 cells were cultured in Iscove's Modified DMEM supplemented with 10\% Foetal Bovine Serum (FBS; Gibco Thermo Fisher Scientific), 2mM L-glutamine (200mM Gibco Thermo Fisher Scientific), 1\% Sodium Pyruvate (100X Gibco Thermo Fisher Scientific), 1\% Non-Essential Amino Acids (100X Gibco Thermo Fisher Scientific) and 1\% 1X Antibiotic-Antimycotic (100X Gibco Thermo Fisher Scientific). LX2 cells were cultured under standard conditions in a humidified incubator at $37{ }^{\circ} \mathrm{C}$ with $5 \% \mathrm{CO}_{2}$. Once cells reached $\sim 75 \%$ confluence, cells were trypsinised using $0.25 \%$ Trypsin-EDTA (Thermo Fisher Scientific) and passaged at a split ratio of $1: 3$.

Vials of primary human hepatocytes (plateable hepatocytes, Thermo Fisher Scientific, catalogue number HMCPMS) were directly used for bio-printing as further described in the paragraph 3.3.4 of this Chapter.

\subsubsection{Printability tests of human liver ECM solution combined with Cellink bio-ink at different ratios}

The printability of human liver ECM solution was evaluated in combination with Cellink bio-ink which is a nanocellulose based bio-ink provided by CELLINK AB at different ratios, respectively ECM solution:Cellink bio-ink 20:80, 30:70 and 50:50.

$\mathrm{ECM}+$ Cellink bio-inks at different ratios were prepared by mixing the desired volume of liver ECM solution and Cellink bio-ink using $10 \mathrm{ml}$ Luer lock syringes (CELLINK AB) linked by a syringe connector (Cole Palmer). The homogeneous bio-inks were then transferred to a $3 \mathrm{ml}$ cartridge and loaded in an INKREDIBLE 3D Bio-printer (CELLINK AB) for the bio-printing tests. 
Printability of resultant $\mathrm{ECM}+$ Cellink bio-inks was investigated by performing the following defined parameters/analysis as indicated by CELLINK AB group: 1) line test, 2) spiral test and 3) bio-printing of a cube sample (Figure 30).

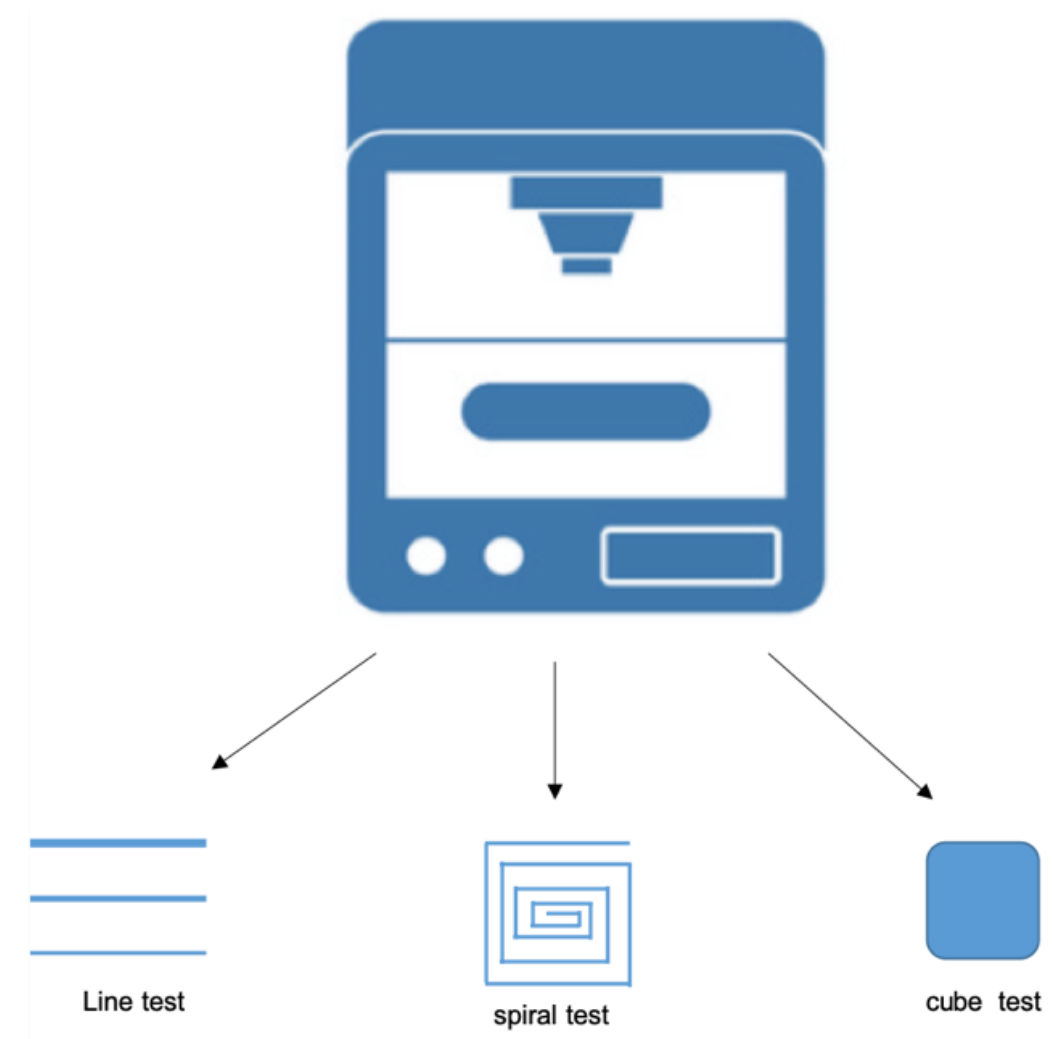

Figure 31. Schematic view of performed bio-printed tests to investigate the printability of human liver ECM solution in combination with Cellink bio-ink at different ratios.

\subsubsection{Line test}

The Line test for the ECM+Cellink bio-ink, composed by human liver ECM solution and Cellink bio-ink, in a ratio 20:80 was performed using a 22G nozzle and a constant extrusion pressure of $17 \mathrm{KPa}$. Different bio-printing speeds were tested, more specifically $450 \mathrm{~mm} / \mathrm{min}, 650 \mathrm{~mm} / \mathrm{min}, 750 \mathrm{~mm} / \mathrm{min}, 900 \mathrm{~mm} / \mathrm{min}$ and $1200 \mathrm{~mm} / \mathrm{min}$. Whilst it is not known the exact rate of fluid dispensation, it is likely to be of the order of microlitres per second.

Line test for the ECM+Cellink bio-ink, composed by human liver ECM solution and Cellink bio-ink, in a ratio $30: 70$ was performed using a $22 \mathrm{G}$ nozzle and a constant 
extrusion pressure of $10 \mathrm{KPa}$. Different bio-printing speeds were tested, more specifically $450 \mathrm{~mm} / \mathrm{min}, 650 \mathrm{~mm} / \mathrm{min}, 750 \mathrm{~mm} / \mathrm{min}$ and $900 \mathrm{~mm} / \mathrm{min}$.

Line test for the ECM+Cellink bio-ink composed by human liver ECM solution and Cellink bio-ink in a ratio 50:50 was performed using a $22 \mathrm{G}$ nozzle and a constant extrusion pressure of $5 \mathrm{KPa}$. Different bio-printing speed were tested, more specifically $450 \mathrm{~mm} / \mathrm{min}, 650 \mathrm{~mm} / \mathrm{min}, 750 \mathrm{~mm} / \mathrm{min}$ and $900 \mathrm{~mm} / \mathrm{min}$.

\subsubsection{Spiral test}

Spiral test for the ECM+Cellink bio-ink composed by human liver ECM solution and Cellink bio-ink in a ratio 20:80 was performed using a 22G nozzle, constant extrusion pressure of $13 \mathrm{KPa}$ and constant bio-printing speed of $600 \mathrm{~mm} / \mathrm{min}$.

Spiral test for the ECM+Cellink bio-ink composed by human liver ECM solution and Cellink bio-ink in a ratio 30:70 was performed using a 22G nozzle, constant extrusion pressure of $10 \mathrm{KPa}$ and constant bio-printing speed of $600 \mathrm{~mm} / \mathrm{min}$.

Spiral test for the ECM+Cellink bio-ink composed by human liver ECM solution and Cellink bio-ink in a ratio 50:50 was performed using a 22G nozzle, constant extrusion pressure of $5 \mathrm{KPa}$ and constant bio-printing speed of $800 \mathrm{~mm} / \mathrm{min}$.

\subsubsection{Bio-printing of cube samples}

Bio-printing of a cube sample of $10 \times 10 \mathrm{~mm}$ with a 2 bio-printing layers thickness, using $\mathrm{ECM}+$ Cellink bio-ink composed by human liver ECM solution and Cellink bio-ink in a ratio 20:80, was performed using a $22 \mathrm{G}$ nozzle, constant extrusion pressure of $17 \mathrm{KPa}$ and constant bio-printing speed of $600 \mathrm{~mm} / \mathrm{min}$.

Bio-printing of a cube sample of $5 \times 5 \mathrm{~mm}$ with a 3 bio-printing layers thickness, using $\mathrm{ECM}+$ Cellink bio-ink composed by human liver ECM solution and Cellink bio-ink in a ratio 30:70, was performed using a $22 \mathrm{G}$ nozzle, constant extrusion pressure of $6 \mathrm{KPa}$ and constant bio-printing speed of $600 \mathrm{~mm} / \mathrm{min}$. 
Bio-printing of a cube sample test of $3 \times 3 \mathrm{~mm}$ with a 3 bio-printing layers thickness, using ECM+Cellink bio-ink composed by human liver ECM solution and Cellink bioink in a ratio 50:50 was performed using a $22 \mathrm{G}$ nozzle, constant extrusion pressure of $5 \mathrm{KPa}$ and constant bio-printing speed of $600 \mathrm{~mm} / \mathrm{min}$.

\subsubsection{Bio-printing of human liver cells}

The previously mentioned bio-printing tests showed that the ratio of 50:50 was the most optimal ratio to prepare bioengineered bio-printing samples containing human cells, because at this ratio of 50:50 it was possible to bio-print with a very low extrusion pressure and obtain $3 \mathrm{D}$ constructs with a well define shape. For this reason, the ratio 50:50 was selected to perform all the experiments further described in this Chapter.

For all tested human liver cells, the first step consisted in the preparation of ECM+Cellink bio-ink composed by human liver ECM solution and Cellink bio-ink in ratio 50:50. This step was carried out by several mixing cycles of the desired volume of liver ECM solution and Cellink bio-ink using $10 \mathrm{ml}$ Luer lock syringes (CELLINK AB) linked by a syringe connector (Cole Palmer).

Cellink bio-ink only was used as internal control to evaluate if the presence of liver ECM components would enhance cell viability, proliferation and functionality. Resultant bioengineered samples bio-printed using solely Cellink bio-ink were called Cellink bio-ink samples.

HepG2 or LX2 cells were then trypsinised and counted to obtain a total amount of cells corresponding to the desired cell density of 10 million cells per millilitre $(\mathrm{ml})$ of bio-ink. After centrifugation, the required cell amount was resuspended in complete medium (100 $\mu \mathrm{l}$ of cell resuspension per $\mathrm{ml}$ of bio-printed bio-ink) and then transferred to a 1 $\mathrm{ml}$ syringe (CELLINK AB) and mixed with ECM+Cellink bio-ink or Cellink bio-ink only utilizing the CELLMIXER device (CELLINK AB). The homogeneous bio-ink containing the cell line was transferred to a $3 \mathrm{ml}$ cartridge and loaded in an INKREDIBLE 3D Bioprinter (CELLINK AB) for printing the samples under physiological conditions, such as room temperature and low extrusion pressure 6kPa versus 20-24 kPa (table 8 and 9). 
Bio-printing of HepG2 and LX2 cells

Values

using ECM+Cellink Bio-ink

\begin{tabular}{|c|c|}
\hline Bio-printing speed & $1200 \mathrm{~mm} / \mathrm{min}$ \\
\hline Nozzle & $22 \mathrm{G}$ \\
\hline Pressure & $6 \mathrm{kPa}$ \\
\hline Temperature & Room Temperature \\
\hline Cell density & $10 \times 10^{6} \mathrm{cell} / \mathrm{ml}$ bio-ink \\
\hline Sample shape & $6 \times 6 \mathrm{~mm}$ and 2 printed layers thickness \\
\hline Plate Dimension & 12 well plate \\
\hline
\end{tabular}

Table 8. Detailed parameters used for the bio-printing of single cell samples of HepG2 or LX2 using ECM+Cellink bio-ink in ratio 50:50.

\begin{tabular}{|c|c|}
\hline $\begin{array}{c}\text { Bio-printing of HepG2 and LX2 cells } \\
\text { using Cellink Bio-ink } \\
\text { Bio-printing speed }\end{array}$ & Values \\
\hline Nozzle & $1200 \mathrm{~mm} / \mathrm{min}$ \\
\hline Pressure & $22 \mathrm{G}$ \\
\hline Temperature & $20-24 \mathrm{kPa}$ \\
\hline Cell density & Room Temperature \\
\hline Sample shape & $10 \times 10^{6}$ cell/ml bio-ink \\
\hline Plate Dimension & $6 \times 6 \mathrm{~mm}$ and 2 printed layers thickness \\
\hline
\end{tabular}

Table 9. Detailed parameters used for the bio-printing of single cell samples of HepG2 or LX2 using Cellink bio-ink only.

In the case of primary human hepatocytes, cells were thaw in a water bath set at 37 ${ }^{\circ} \mathrm{C}$. Considering the high cost of purchasing primary human hepatocytes and the 
knowledge that LX2 and HepG2 cell viability and activity was higher when using ECMCellink bio-ink compared to Cellink bio-ink only, the bio-printing of primary human hepatocytes was performed only using ECM+Cellink bio-ink. Cell suspension and hepatocyte thawing medium was used (Cryopreserved Hepatocytes Recovery Medium, Thermo Fisher Scientific). Cells were transferred in a $50 \mathrm{ml}$ tube (Starlab) and centrifuged (Heraeus Instruments, Megafuge $1.0 \mathrm{R}$ ) at $100 \mathrm{xg}$ for 10 minutes. Supernatant was discarded and the cell pellet was resuspended in $200 \mu \mathrm{l}$ of hepatocytes plating medium (Williams' Medium E no phenol red, Thermo Fisher Scientific) supplemented with Hepatocyte Plating Supplement Pack (Thermo Fisher Scientific). This was followed by cell counting using trypan blue (Sigma-Aldrich) to distinguish alive cells from dead cells. The cell suspension was resuspended in the appropriate volume of ECM+Cellink bio-ink to obtain the cell density of 9 million cells per millilitre $(\mathrm{ml})$ of bio-ink. To do so, cell resuspension was then transferred to a $1 \mathrm{ml}$ syringe (CELLINK $A B$ ) and mixed with ECM+Cellink bio-ink utilizing the CELLMIXER device (CELLINK AB). The homogeneous ECM+Cellink bio-ink containing human primary hepatocytes was transferred to a $3 \mathrm{ml}$ cartridge and loaded in an INKREDIBLE 3D Bio-printer (CELLINK AB) to print the samples under physiological conditions, such as room temperature and an extrusion pressure of $6 \mathrm{kPa}$ (table 10).

\begin{tabular}{|cc|}
\hline $\begin{array}{c}\text { Bio-printing of primary human } \\
\text { hepatocytes using ECM+Cellink bio-ink }\end{array}$ & Values \\
\hline Bio-printing speed & $1200 \mathrm{~mm} / \mathrm{min}$ \\
\hline Nozzle & $22 \mathrm{G}$ \\
\hline Pressure & $6 \mathrm{kPa}$ \\
\hline Temperature & Room Temperature \\
\hline Cell density & $9 \times 10^{6} \mathrm{cell} / \mathrm{ml}$ bio-ink \\
\hline Sample shape & Drops $(50 \mu \mathrm{ll}$ of bio-ink $)$ \\
\hline Plate Dimension & 48 well plate \\
\hline
\end{tabular}

Table 10. Detailed parameters used for the bio-printing of primary human hepatocytes samples using ECM+Cellink bio-ink in ratio 50:50. 


\subsubsection{Cell culture in 3D bio-printed samples}

Following the bio-printing process, the resulting samples were crosslinked with 100 $\mathrm{mM}$ calcium chloride solution ( $\mathrm{CaCl}_{2}$, Sigma-Aldrich) for 5 minutes. Excess of $\mathrm{CaCl}_{2}$ solution was thereafter removed and the bio-printed samples were washed with HBSS $1 \mathrm{X}$ two consecutive times prior to the addition of $1 \mathrm{ml}$ of the cell specific complete medium.

HepG2 bio-printed samples were cultured in MEM Medium supplemented with 10\% Foetal Bovine Serum, 2mM L-glutamine, 1\% Sodium Pyruvate, 1\% Non-Essential Amino Acids and 1\% 1X Antibiotic-Antimycotic. HepG2 bio-printed samples were cultured in a humidified incubator at $37{ }^{\circ} \mathrm{C}$ with $5 \% \mathrm{CO}_{2}$ up to 13 days replacing the complete medium every other day.

LX2 bio-printed samples were cultured in Iscove's Modified DMEM supplemented with 10\% Foetal Bovine Serum 2mM L-glutamine, 1\% Sodium Pyruvate, 1\% Non-Essential Amino Acids and 1\% 1X Antibiotic-Antimycotic. LX2 bio-printed samples were cultured in a humidified incubator at $37^{\circ} \mathrm{C}$ with $5 \% \mathrm{CO}_{2}$ up to 13 days replacing the complete medium every other day. At 7 days of cell culture, four LX2 bio-printed samples were treated with TGF $\beta 15 \mathrm{ng} / \mathrm{ml}$ for 6 days, refreshing every other day (3x48hrs treatment).

Primary human hepatocytes bio-printed samples were cultured for the first 24 hours in hepatocytes plating medium which was then changed into hepatocyte incubation medium (Williams' Medium E no phenol red, Thermo Fisher Scientific) supplemented with Hepatocyte Maintenance Supplement Pack (Thermo Fisher Scientific). Primary human hepatocytes bio-printed samples were cultured in a humidified incubator at 37 ${ }^{\circ} \mathrm{C}$ with $5 \% \mathrm{CO}_{2}$ up to 10 days replacing the complete medium every other day.

During cell culture, cell supernatants were collected and stored at $-20{ }^{\circ} \mathrm{C}$ for further evaluation. 


\subsubsection{Live/Dead cell double staining}

Cell viability was measured employing the Live/Dead fluorescence double staining assay. This technique is based on the use of Propidium lodide (PI) (Sigma-Aldrich), an intercalating red fluorescent agent that binds only to the DNA of apoptotic cells since it cannot cross the membrane of living cells, and Fluorescein Diacetate (FDA) (Sigma-Aldrich), a non-fluorescent agent that in the nucleus of alive cells is converted to fluorescein, a green fluorescent compound.

Live/Dead double staining was performed at day 7 and 13 in the case of LX2 and HepG2 bio-printed samples while for the primary hepatocytes bio-printed samples Live/Dead double staining assay was performed at day 1 and 10.

Live/Dead cell double staining assay was performed by adding $500 \mu$ of pre-warmed 1X HBSS (Gibco) containing 4\% Propidium lodide (PI) and 2\% Fluorescein Diacetate (FDA) to each bio-printed sample. This was followed by incubation in the dark for 1 minute at room temperature and consequent several washes with HBSS $1 \mathrm{X}$ to remove any non-adherent dyes. Samples were then visualised under fluorescent microscope (Nikon model U-III equipped with Nikon mercury lamp) and analysis of the captured images was performed with Fiji v1.49d (ImageJ Jenkins server).

\subsubsection{Alamar blue viability test}

AlamarBlue $\AA$ (Thermo Fisher Scientific) cell viability reagent 10X was first diluted 1:10 with cell culture medium and then $1.4 \mathrm{ml}$ of this solution was added to each bioengineered bio-printed sample. Samples were then incubated for 4 hours in a humidified incubator at $37^{\circ} \mathrm{C}$ with $5 \% \mathrm{CO}_{2}$. Quadruplicates of each sample $(200 \mu \mathrm{l} \mathrm{per}$ well) were analysed in a 96 opaque black well plate. A Microplate Reader (Infinite M200 PRO Multimode Microplate reader, Tecan, Switzerland) was used to measure the absorbance at $570 \mathrm{~nm}$. 


\subsubsection{Histology}

The bioengineered bio-printed samples, previously fixed in 10\% formalin (Leica Biosystems), were first washed in distilled water and then dehydrated in a series ethanol (Acquascience) and xylene baths, followed by embedding in paraffin (Leica Biosystems). The samples were sliced into $5 \mu \mathrm{m}$ sections using a microtome (Leica Biosystems Leica RM2035). All sections were then immersed in xylene baths (Acquascience) for at least 5 minutes, and ethanol baths (Acquascience) for at least 2 minutes and then rinsed in tap water.

Sections were first stained with Harris Hematoxylin (Leica Biosystems) for 10 minutes followed by washing in tap water for 5 minutes. Next, the sections were stained with Eosin (Leica Biosystems) for 3 minutes, followed by washing in tap water for 5 minutes. The sections were then quickly dehydrated in ethanol and then immersed in xylene bath (Acquascience) until mounted with DPX (Leica Biosystems) and finally cover slipped. Images were captured with an Axiocam IcC5 using Zeiss Axiovision (version 4.8.2).

\subsubsection{Human Albumin ELISA}

Content of secreted albumin by human primary hepatocytes or HepG2 cell supernatants was quantified by performing the Human Albumin ELISA assay (Human Albumin ELISA kit ABCAM). Cell supernatants, collected at different time points during cell culture, were diluted 1:60 for HepG2 samples or 1:10 for human primary hepatocytes samples with Diluent Solution provided in the kit. Fifty $\mu \mathrm{l}$ of diluted cell supernatants and Albumin Standard Solutions were added and incubated in a 96 well plate for 1 hour followed by 5 washes with Washing Buffer. Fifty $\mu$ l of $1 \mathrm{X}$ Biotinylated Albumin Antibody was then added in each well and washed after 30 minutes. Next, 1X SP Conjugate antibody was added and washed after 30 minutes. Next, $50 \mu \mathrm{l}$ of Chromogen Substrate Solution was added in each well and incubated for 25 minutes. Last, $50 \mu \mathrm{l}$ of Stop Solution was used to block the reaction until the colour of the 
samples turned from blue to yellow. Absorbance signal related to albumin content was measured at $450 \mathrm{~nm}$ using the plate reader (FLUOstar Omega BMG Labtech).

\subsubsection{Lactate Dehydrogenase (LDH) Cytotoxicity Assay}

The cytotoxicity of bio-printed human primary hepatocytes was investigated using Pierce Lactate Dehydrogenase (LDH) Cytotoxicity Assay Kit (Thermo Fisher Scientific). Lactate dehydrogenase is a cytosolic enzyme present in specific cell types including the human primary hepatocytes. Following plasma membrane damage the LDH is released into the cell culture media and the level of this enzyme can be quantified by a coupled enzymatic reaction in which LDH first converts lactate to pyruvate via NAD+ reduction to NADH. In the second enzymatic reaction, Diaphorase, a flavin-bound enzyme, uses NADH to catalyse the reduction of tetrazolium salt to a red formazan product that can be measured at $490 \mathrm{~nm}$. The level of formazan formation is directly proportional to the amount of LDH released into the medium. Cytotoxicity of human primary hepatocytes was analysed by measuring the concentration of LDH in the cell supernatant collected at day 3, 5, 7 and 10 of cell culture. Fifty $\mu \mathrm{L}$ of cell supernatants were transferred to a 96 -well flat-bottom plate (each sample in triplicate). Next, $50 \mu \mathrm{L}$ of Reaction Mixture was added to each sample and mixed by pipetting. This was followed by incubation at room temperature for 30 minutes protected from light. Lastly, $50 \mu \mathrm{L}$ of Stop Solution was added to each sample and mixed by gentle tapping. Absorbance signal related to LDH content was read at $490 \mathrm{~nm}$ using the plate reader (FLUOstar Omega BMG Labtech).

\subsubsection{TGF- $\beta 1$ Treatment}

Bio-printed LX2 samples using ECM+Cellink bio-ink or Cellink bio-ink were employed to investigate the pro-fibrotic effect of TGF- $\beta 1$ treatment. LX2 cells were cultured for 7 days before being exposed to TGF- $\beta 1$ ( $5 \mathrm{ng} / \mathrm{ml}, \mathrm{R} \& D$ Systems). Treatment of the 
samples $(n=5)$ with TGF- $\beta 1$ was performed for 6 days replacing the dose every 48 hours. This was followed by quantification of secreted pro-Collagen 1 and gene expression analysis to evaluate differences between TGF- $\beta 1$ treated samples and untreated control samples ( $n=4$ for both TGF- $\beta 1$ treated and untreated samples).

\subsubsection{Human Pro-Collagen 1 alpha 1 ELISA}

Content of secreted pro-collagen in LX2 cell supernatants was quantified by performing Human Pro-Collagen 1 alpha 1 ELISA assay (Human Pro-Collagen 1 alpha 1 ELISA kit ABCAM). Cell supernatants, collected at different time points during cell culture, were diluted 1:60 with Diluent Solution provided in the kit. $50 \mu$ of diluted cell supernatants and Pro-Collagen 1 Standard Solutions were added in a 96 well plate. Fifty $\mu \mathrm{l}$ of Antibody Cocktail was added to each well containing the samples or standard solutions followed by a 1 hour incubation at room temperature on a plate shaker (Ika Labortechnik KS501 digital) set at $400 \mathrm{rpm}$. Each well was then washed 3 times with 1X Wash Buffer. $100 \mu \mathrm{l}$ of TMB Development Solution was added to each well and incubated for 10 minutes in the dark on a plate shaker set at $400 \mathrm{rpm}$. Next $100 \mu \mathrm{l}$ of Stop solution was added to each well followed by 1 minute incubation on a plate shaker (Ika Labortechnik KS501) Absorbance signal related to Pro-Collagen 1 content was read at $450 \mathrm{~nm}$ using the plate reader (FLUOstar Omega BMG Labtech).

\subsubsection{RNA extraction, reverse transcription and gene expression analysis}

\subsubsection{RNA extraction}

RNeasy Plant Kit (Qiagen) was employed to extract RNA from the LX2 and HepG2 cells grown in bio-printed samples using ECM+Cellink bio-ink or Cellink bio-ink only.

Lysis Buffer (Qiagen) containing $1 \%$ of $\beta$-mercaptoethanol (Sigma-Aldrich) was first added to the previously snap frozen bio-printed samples followed by vigorously 
pipetting to further disrupt the samples. The main reason for using the Plant kit was the necessity to remove the cellulose present in the hydrogels in order to achieve the maximum yield of RNA. This step was performed by using the QIAshredder column provided by the manufacturer. As indicated in the protocol provided by the manufacturer, subsequent steps were performed using spin columns and different ethanol-based buffers. RNA extracted from Hep-Gel was measured at $260 \mathrm{~nm}$ with the spectrophotometer (NanoDrop, ThermoScientific).

\subsubsection{Complementary DNA (cDNA) synthesis}

Complementary cDNA reverse transcription was performed using high Capacity cDNA Reverse Transcription Kit (Applied Biosystems). The extracted RNA was diluted in RNase free water (Qiagen) in order to obtain the same RNA concentration in all the samples. Subsequently, $10 \mu \mathrm{l}$ of the samples' RNA was added in independent PCR microtubes and then mixed with $10 \mu$ of Master Mix, which composition is shown in the table 11. The reverse transcription was performed using the 2720 Thermal Cycler (Applied Biosystems) following the program described in table 12.

\begin{tabular}{|l|l|}
\hline RT Master Mix reagents & Volume $(\boldsymbol{\mu L})$ \\
\hline 10X RT Buffer & 2 \\
\hline 25X dNTPs Mix (100 mM) & 0.8 \\
\hline 10X RT Random Primers & 2 \\
\hline MultiScribe RT & 1 \\
\hline RNase Inhibitor & 1 \\
\hline Nuclease Free $\mathrm{H}_{\mathbf{2}} \mathbf{O}$ & 3.2 \\
\hline
\end{tabular}

Table 11. RT Master Mix composition. 


\begin{tabular}{|l|l|}
\hline Step 1 & 10 minutes at $25^{\circ} \mathrm{C}$ \\
\hline Step 2 & 120 minutes at $37^{\circ} \mathrm{C}$ \\
\hline Step 3 & 5 minutes at $85^{\circ} \mathrm{C}$ \\
\hline Step 4 & Hold at $4^{\circ} \mathrm{C}$ \\
\hline
\end{tabular}

Table 12. Steps of Reverse transcription program using 2720 Thermal Cycler.

\subsubsection{Quantitative Real-Time PCR (RT- qPCR)}

The obtained cDNA was first diluted in RNase free water to get a final concentration of $2 \mathrm{ng} / \mu \mathrm{l} .10 \mathrm{ng}$ of cDNA from each Hep-gel was added into a Fast Optical 96-well TaqMan PCR Plate (MicroAmp, Applied Biosystems) and then mixed with $15 \mu \mathrm{l}$ of qPCR Master Mix, which composition is described in table 13. Last, the PCR Plate was inserted in ABI 7500 Fast Real Time PCR System (Applied Biosystems) to perform 40 cycles of cDNA amplification (table 14).

Comparative CT method was used to analyse the relative expression of the investigated genes (table 15) using Glyceraldeyde-3-phosphate dehydrogenase (GAPDH) as a housekeeping gene [217].

\begin{tabular}{|l|l|}
\hline RT-qPCR master mix reagents & Volume $(\mu L)$ \\
\hline TaqMan Gene Assay-FAM & 1 \\
\hline TaqMan Univ PCR MM (2X), w/UNG & 10 \\
\hline $\mathbf{H}_{2} \mathbf{O}$ (nuclease free Water) & 4 \\
\hline
\end{tabular}

Table 13. RT-qPCR master mix composition. 


\begin{tabular}{|c|c|}
\hline Step 1 & 2 minutes at $50{ }^{\circ} \mathrm{C}$ \\
\hline Step 2 & 10 minutes at $95^{\circ} \mathrm{C}$ \\
\hline Step 3 & 5 minutes at $95^{\circ} \mathrm{C}$ for 40 cycles \\
\hline Step 4 & 11 minutes at $60^{\circ} \mathrm{C}$ \\
\hline
\end{tabular}

Table 14. Program used for Quantitative Real-Time PCR (RT- qPCR) using ABI 7500 Fast Real Time PCR System.

\begin{tabular}{|l|l|l|}
\hline Gene & NCBI Ref. Seq. & ID Number \\
\hline GAPDH & NM_001256799.2 & Hs02786624_g1 \\
\hline AFP & NM_001134.2 & Hs01040598_m1 \\
\hline ALB & NM_000477.5 & Hs00910225_m1 \\
\hline HNF4A & NM_000457.4 & Hs00230853_m1 \\
\hline COL1A1 & NM_000088.3 & Hs00164004_m1 \\
\hline ACTA2 & NM_001141945.2 & Hs00426835_g1 \\
\hline LOX & NM_001178102.2 & Hs00942480_m1 \\
\hline
\end{tabular}

Table 15. List of TaqMan assays on demand used (Lifescience Technologies).

\subsubsection{Statistics and data analysis}

Paired t-test was performed to evaluate statistical differences between samples bioprinted using ECM+Cellink bio-ink and Cellink bio-ink only. SPSS v21 software was used to perform the statistical analysis. 


\subsection{Results}

\subsubsection{Assessing printability of human liver ECM solution combined with Cellink bio-ink at different ratios}

The printability tests were carried out to assess the feasibility of bio-printing human liver ECM solution together with Cellink bio-ink at different ratios, respectively ECM solution:Cellink bio-ink 20:80, 30:70 and 50:50. Performed printability tests included line test, spiral test and bio-printing of a cube sample using CELLINK INKREDIBLE bio-printer. Considering the fact that cells would be bio-printed in 3D constructs, the most important part of these tests was to define the optimal ECM solution:Cellink bioink ratio to produce cube samples.

\subsubsection{Bio-printing of liver ECM solution:Cellink in ratio 20:80}

Considering that the concentration of human liver ECM solution was 5,3 mg, the ECM concentration of the ECM+Cellink bio-ink in ratio $20: 80$ was $1,06 \mathrm{mg} / \mathrm{ml}$.

Line test (Figure 31) was performed at different bio-printing speed, more specifically $450 \mathrm{~mm} / \mathrm{min}$ (Figure 31 line a), $650 \mathrm{~mm} / \mathrm{min}$ (Figure 31 line b), $750 \mathrm{~mm} / \mathrm{min}$ (Figure 31 line c), $900 \mathrm{~mm} / \mathrm{min}$ (Figure 31 line d) and $1200 \mathrm{~mm} / \mathrm{min}$ (Figure 31 line e). The Line test confirmed the feasibility to precisely and homogeneously dispense the ECM+Cellink bio-ink in ratio 20:80 along a straight line at all tested bio-printing speeds except for the highest speed of $1200 \mathrm{~mm} / \mathrm{min}$ (Figure 31 line e). 


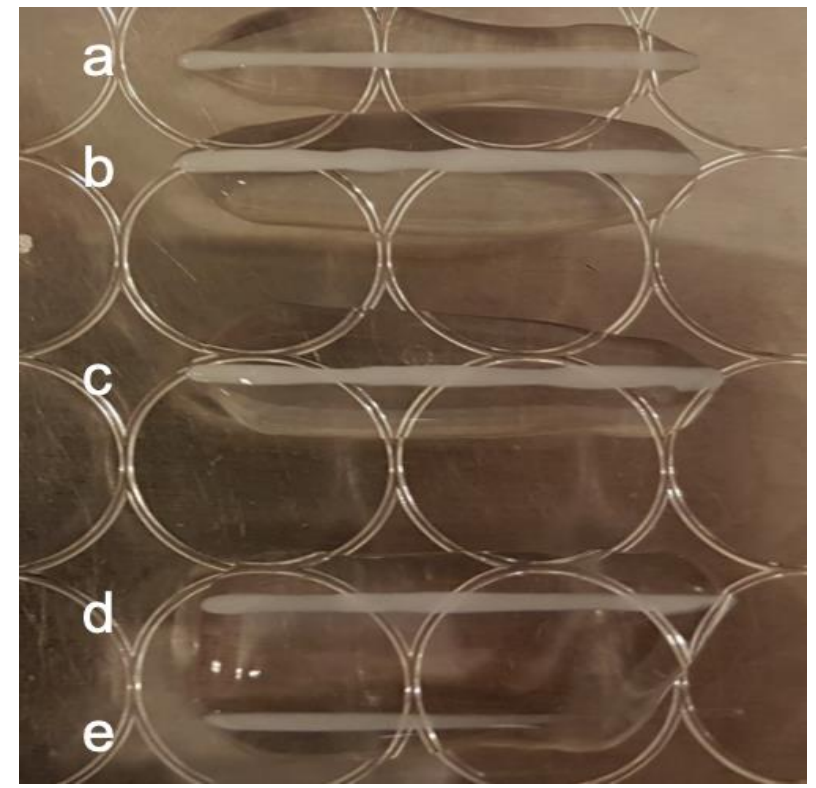

Figure 32. Line deposition test of the ECM+Cellink bio-ink composed by ECM solution and Cellink bio-ink in ratio 20:80 at different bio-printing speed: (a) $450 \mathrm{~mm} / \mathrm{min}$, (b) $650 \mathrm{~mm} / \mathrm{min}$, (c) $750 \mathrm{~mm} / \mathrm{min}$ and (d) $900 \mathrm{~mm} / \mathrm{min}$ and (e) $1200 \mathrm{~mm} / \mathrm{min}$.

Spiral test (Figure 32) using ECM+Cellink bio-ink in ratio 20:80 showed that this bioink was suitable for the bio-printing of more complex samples since it was feasible to change the spatial $X-Y$ direction of the bio-ink dispenser without affecting the accuracy in the bio-ink deposition.

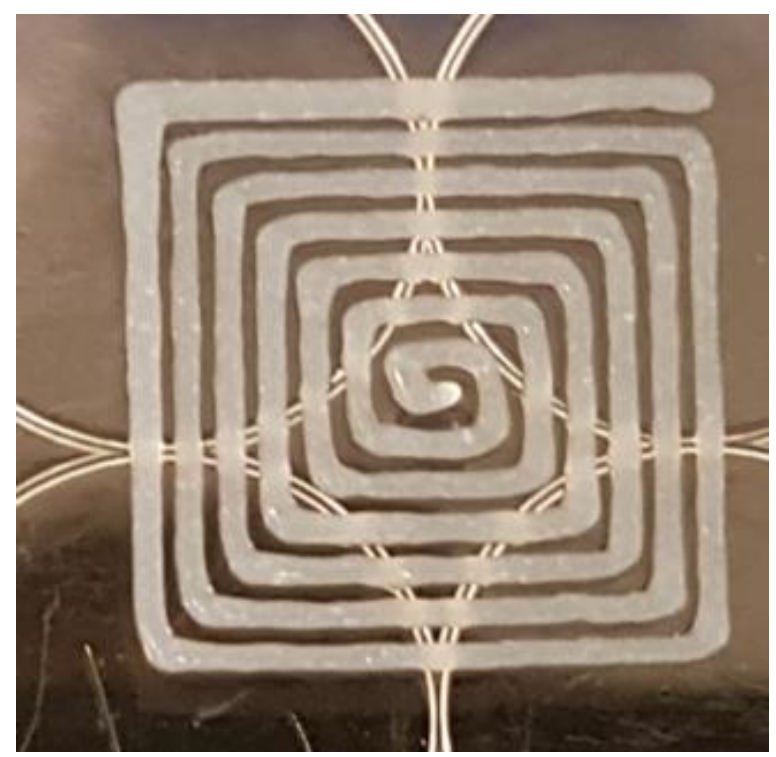

Figure 33. Spiral test using ECM+Cellink bio-ink composed by ECM solution and Cellink bioink in ratio 20:80. 
Bio-printing of a cube sample (Figure 33) of $10 \times 10 \mathrm{~mm}$ with 2 bio-printing layers confirmed the feasibility to precisely dispose/dispense the ECM+cellink bio-ink in ratio 20:80 to obtain the desired sample shape.

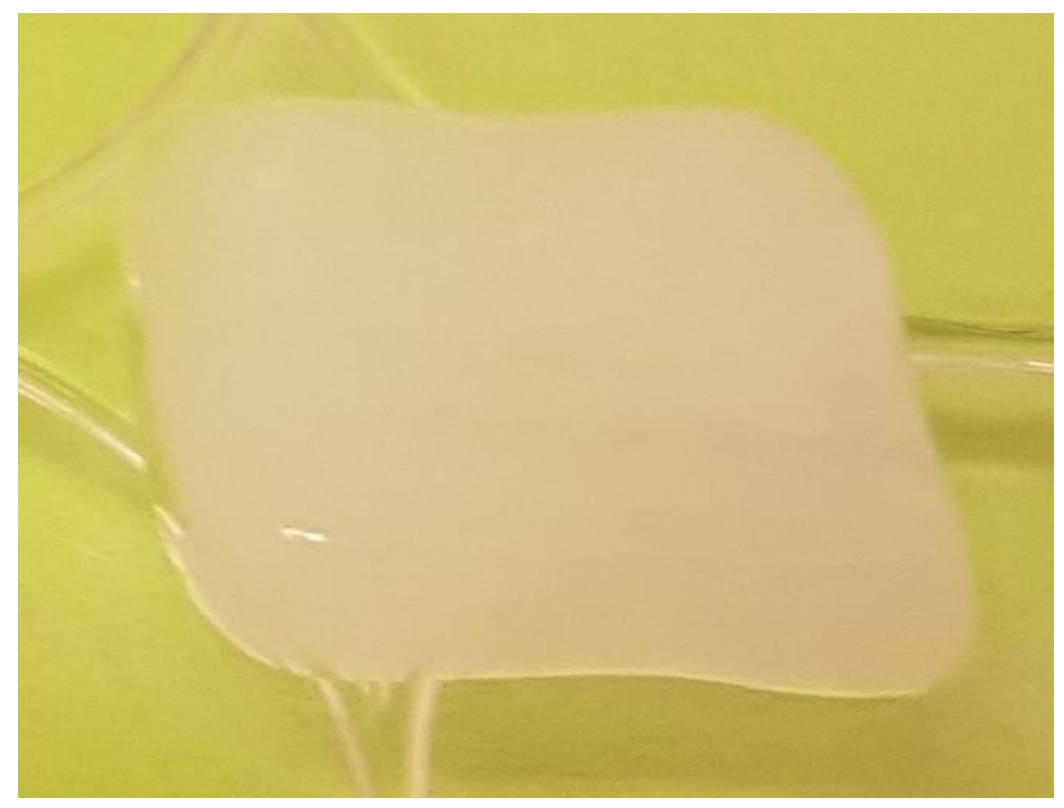

Figure 34. Bio-printing of a sample cube $10 \times 10 \mathrm{~mm}$ with 2 bio-printing layers thickness using ECM+Cellink bio-ink composed by ECM solution and Cellink bio-ink in ratio 20:80.

\subsubsection{Bio-printing of liver ECM solution:Cellink in ratio 30:70}

Considering that the concentration of human liver ECM solution was $5.3 \mathrm{mg}$, the ECM concentration of the ECM+Cellink bio-ink in ratio $30: 70$ was $1.59 \mathrm{mg} / \mathrm{ml}$.

Line test (Figure 34) using ECM+Cellink bio-ink in ratio 30:70 was performed at different bio-printing speeds, specifically $450 \mathrm{~mm} / \mathrm{min}$ (Figure 34 line a), $650 \mathrm{~mm} / \mathrm{min}$ (Figure 34 line b), $750 \mathrm{~mm} / \mathrm{min}$ (Figure 34 line c) and $900 \mathrm{~mm} / \mathrm{min}$ (Figure 34 line d). Line test confirmed the feasibility of a precisely and homogeneously dispense along a straight line the ECM+Cellink bio-ink in ratio 30:70 at all tested bio-printing speeds. 


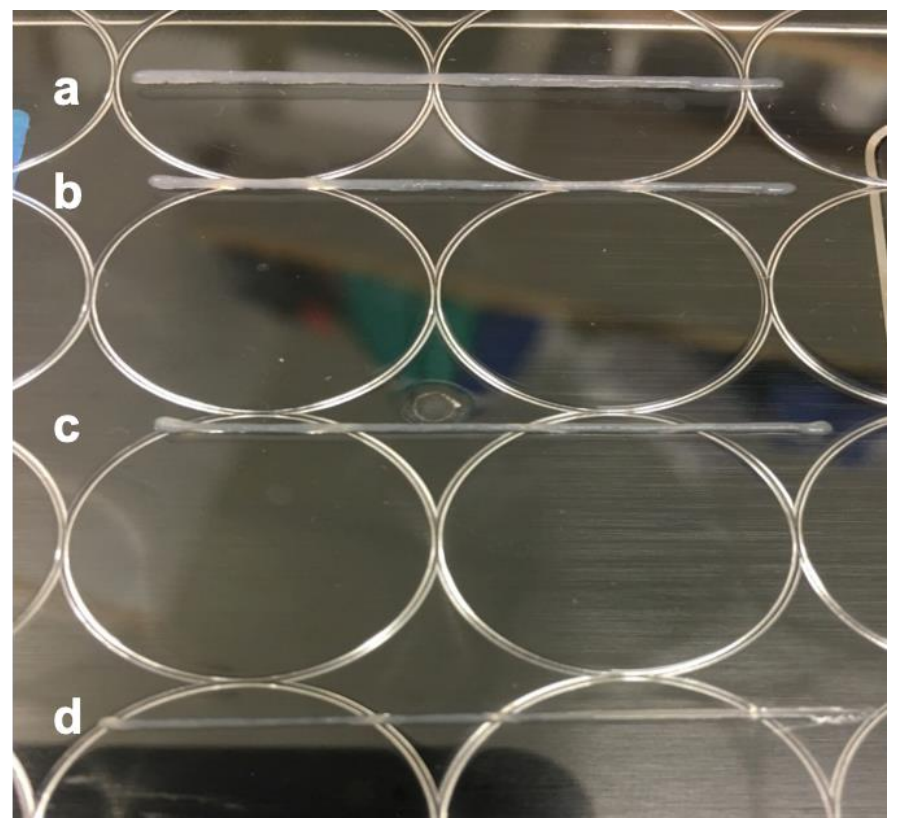

Figure 35. Line deposition test of the ECM+Cellink bio-ink composed by ECM solution and Cellink bio-ink in ratio 30:70 at different bio-printing speed: (a) $450 \mathrm{~mm} / \mathrm{min}$, (b) $650 \mathrm{~mm} / \mathrm{min}$, (c) $750 \mathrm{~mm} / \mathrm{min}$ and (d) $900 \mathrm{~mm} / \mathrm{min}$.

Similar to ECM+Cellink bio-ink in ratio 20:80, the spiral test (Figure 35) for the $E C M+C e l l i n k$ bio-ink in ratio 30:70 showed that this bio-ink composition was suitable for the bio-printing of the spiral sample as it was possible to change the spatial $X-Y$ direction of the bio-ink dispenser without affecting the accuracy in the bio-ink deposition.

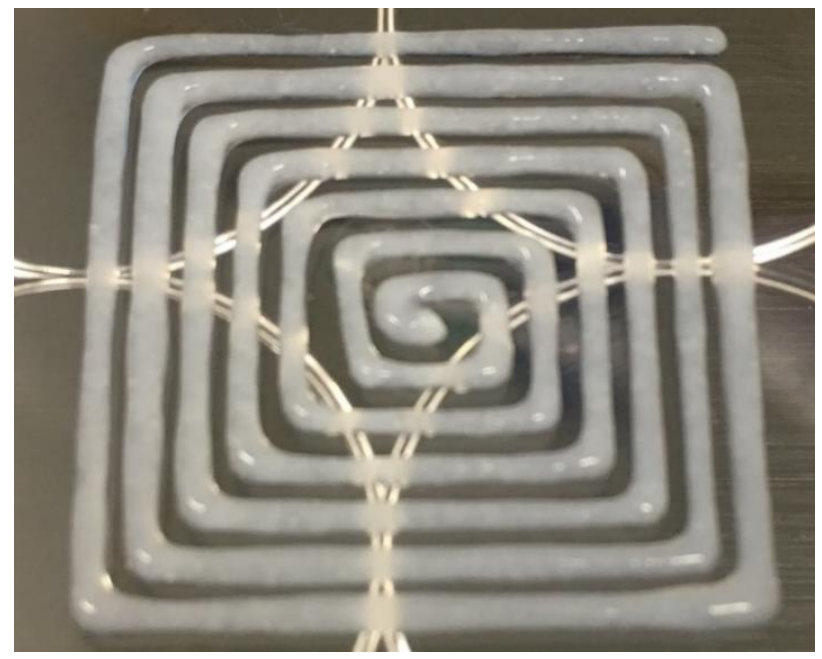

Figure 36. Spiral test using ECM+Cellink bio-ink composed by ECM solution and Cellink bioink in ratio 30:70. 
Bio-printing of a cube sample (Figure 36 ) of $5 \times 5 \mathrm{~mm}$ with 3 bio-printing layers thickness confirmed the feasibility to precisely dispense the ECM+cellink bio-ink in ratio 30:70 to obtain the desired sample shape.

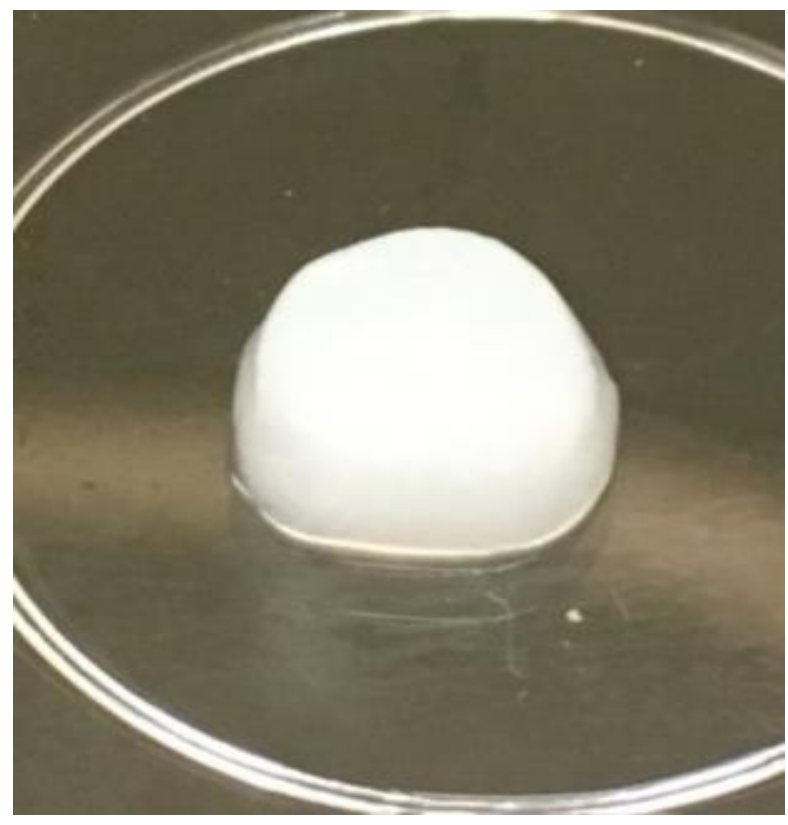

Figure 37. Bio-printing of a sample cube $5 \times 5 \mathrm{~mm}$ with 3 bio-printing layers thickness using ECM+Cellink bio-ink composed by ECM solution and Cellink bio-ink in ratio 30:70.

\subsubsection{Bio-printing of liver ECM solution:Cellink in ratio 50:50}

Considering that the concentration of human liver ECM solution was $5,3 \mathrm{mg}$, the ECM concentration of the ECM+Cellink bio-ink in ratio $50: 50$ was $2,65 \mathrm{mg} / \mathrm{ml}$.

Line test (Figure 37) using this bio-ink composition was evaluated at different bioprinting speed, in specific $450 \mathrm{~mm} / \mathrm{min}$ (Figure 37 line a), $650 \mathrm{~mm} / \mathrm{min}$ (Figure 37 line b), $750 \mathrm{~mm} / \mathrm{min}$ (Figure 37 line c) and $900 \mathrm{~mm} / \mathrm{min}$ (Figure 37 line d). Line test confirmed the feasibility of precisely and homogeneously dispense along a straight line the ECM+Cellink bio-ink in ratio 50:50 at all bio-printing speeds. Due to lower viscosity of this bio-ink composition compared to the previous two, at slow bio-printing speeds the bio-ink was excessively extruded from the dispenser, as suggested by the width of the bio-printed lines (Figure 37 line $a$ and b), which could compromise the accuracy of the construct its shape. Conversely, at high bio-printing speed, the bio-ink 
deposition did not have this problem and it was possible to obtain narrow bio-printed lines (Figure 37 line $c$ and d).

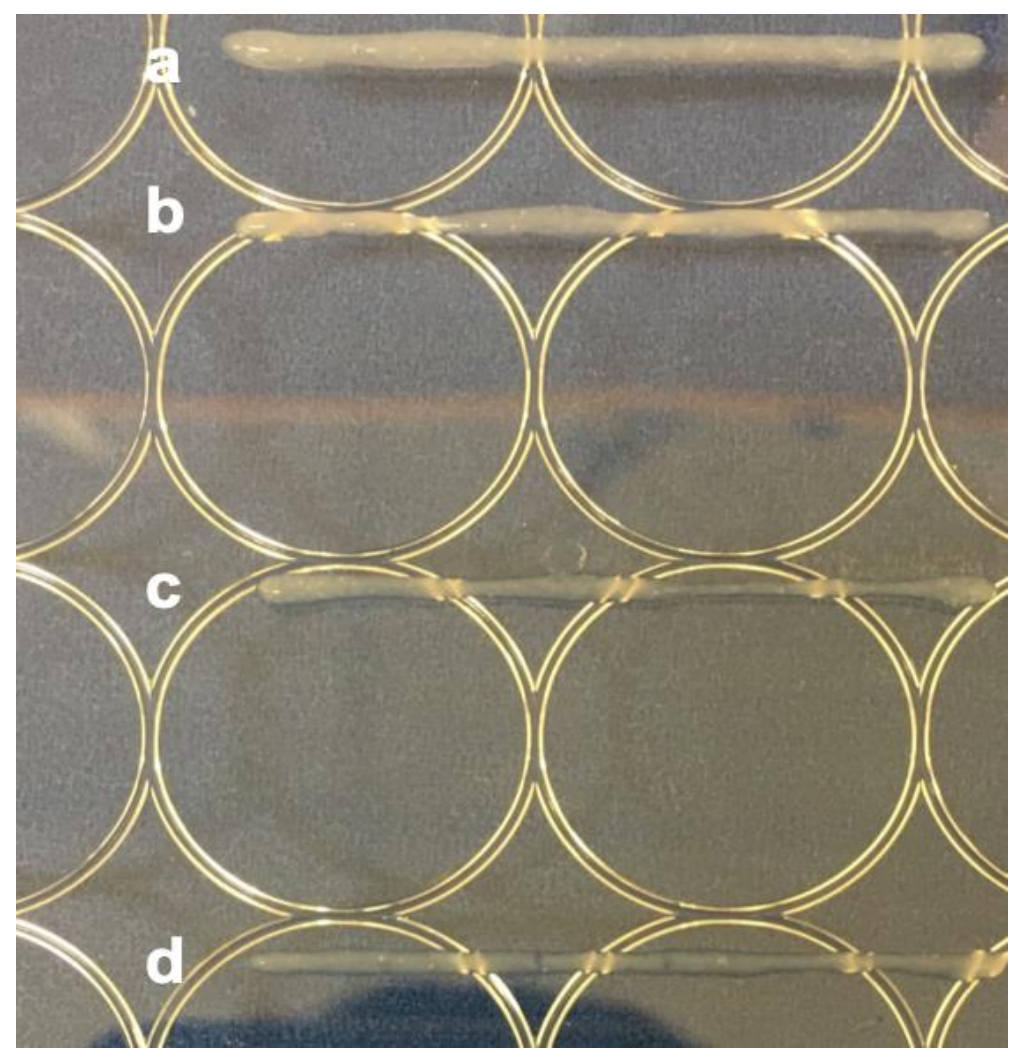

Figure 38. Line deposition test of the ECM+Cellink bio-ink composed by ECM solution and Cellink bio-ink in ratio 50:50 at different bio-printing speed: (a) $450 \mathrm{~mm} / \mathrm{min}$, (b) $650 \mathrm{~mm} / \mathrm{min}$, (c) $750 \mathrm{~mm} / \mathrm{min}$ and (d) $900 \mathrm{~mm} / \mathrm{min}$.

The Spiral test (Figure 38) showed that this bio-ink composition was not suitable for the bio-printing of complex samples since it was not possible to dispense the bio-ink with an adequate spatial precision. As demonstrated the bio-ink was not dispensed homogeneously on the external area of the spiral and, due to the low viscosity of the bio-ink, the parallel lines in the core of the spiral joined together. 


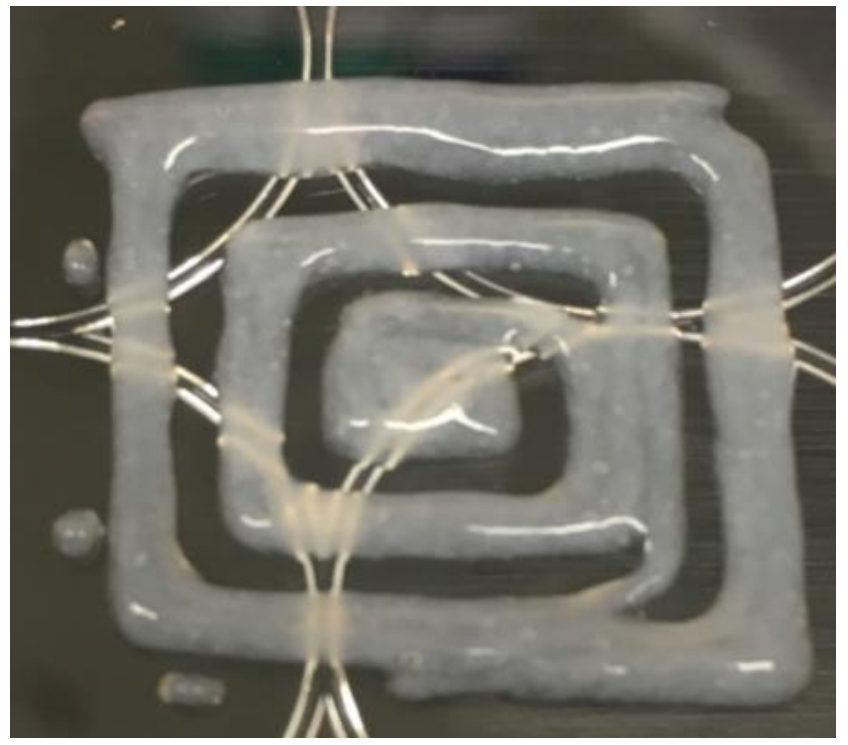

Figure 39. Spiral test using ECM+Cellink bio-ink composed by ECM solution and Cellink bioink in ratio 50:50.

Bio-printing of a cube sample (Figure 39 ) of $3 \times 3 \mathrm{~mm}$ with 3 bio-printing layers thickness confirmed the feasibility of exploiting the ECM+cellink bio-ink in ratio 50:50 to obtain a cube shape.

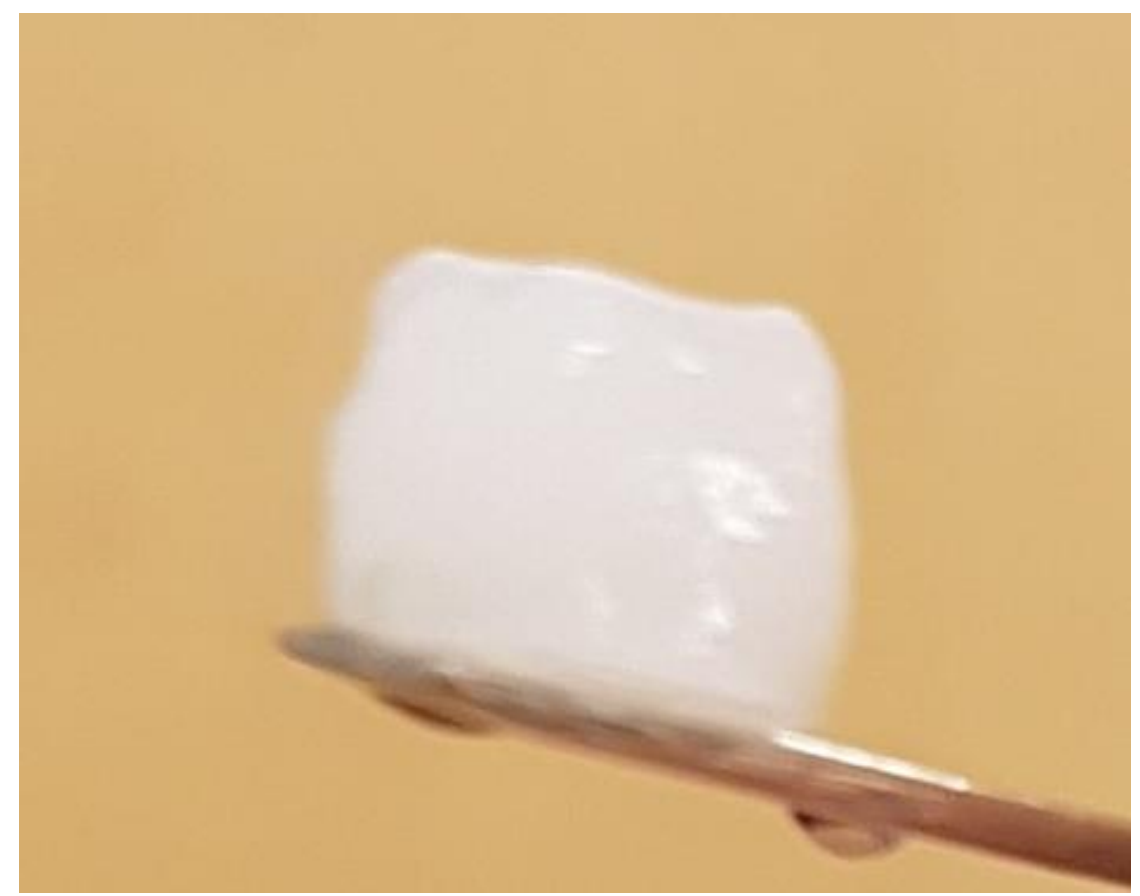

Figure 40. Bio-printing of a sample cube $3 \times 3 \mathrm{~mm}$ and 3 bio-printing layers thickness using ECM+Cellink bio-ink composed by ECM solution and Cellink bio-ink in ratio 50:50. 
Overall, these bio-printing tests showed that the ratio of 50:50 was the most optimal ratio to prepare bioengineered bio-printing samples containing human cells, because it allowed to obtain well defined 3D cubes by applying a very mild extrusion pressure. For this reason, the ratio 50:50 was selected to perform all the experiments further described in this Chapter.

\subsubsection{Bio-printing of human liver cell types}

The in vitro biocompatibility of the ECM+Cellink bio-ink was performed by bio-printing LX2 (a human hepatic stellate cell line), HepG2 (epithelial cells derived from hepatoblastoma) or primary human hepatocytes.

Bio-printing of these human hepatic cells was performed applying the previously mentioned parameters (see section 3.3.6 in Materials and Methods). Briefly, the solubilized ECM was mixed with cellulose-based bio-ink (Cellink ® Bio-ink) in ratio 1:1 as support for bio-printing. Solely Cellink bio-ink was used as internal control to evaluate if the presence of liver ECM components would enhance cell viability, proliferation and functionality.

Different concentrations of $L X 2$ or HepG2 or Human Primary Hepatocytes were gently mixed with ECM+Cellink bio-ink or Cellink bio-ink using a CELLMIXER® and then transferred in a $3 \mathrm{ml}$ cartridge before bio-printing using the INKREDIBLE 3D printer under physiological conditions.

\subsubsection{Bio-printing of LX2 cells}

Chosen shape of bio-printed LX2 samples was a cube of $6 \times 6 \mathrm{~mm}$ with a thickness of 2 bio-printed layers. Total amount of LX2 cells per sample was calculated as 250.000 cells. Bio-printed tissues were maintained in 3D culture up to 13 days and exposed to TGF- $\beta 1(5 \mathrm{ng} / \mathrm{ml})$ for 6 days in order to promote an in vitro pro-fibrogenic process. The resultant bio-printed LX2 samples were analysed by Live/Dead staining, histology, alamarBlue viability assay, gene expression and Pro-Collagen1 ELISA assay. 
Live/Dead staining (Figure 40), of bio-printed LX2 samples was performed at 7 days $(a, c)$ and 13 days $(b, d)$ of cell culture. At 7 days of cell culture, LX2 bio-printed using Cellink bio-ink (a) contained $\sim 50 \%$ living cells and a similar intensity of the green signal (alive cells) and red signal (dead cells) was detected in the captured Live/Dead image. At13 days (b) the cells were non-viable (dead) as indicated by the intense red signal observed in the captured Live/Dead image (b). On the other hand, Live/Dead staining of LX2 bio-printed using ECM+Cellink bio-ink showed a higher number of living cells (green signal) compared to dead cells (red signal) after 7 days in culture (c). At 13 days of cell culture (d) LX2 cells contained $\sim 50 \%$ living cells and a similar intensity of the green signal (alive cells) and red signal (dead cells) was detected in the captured Live/Dead image (d).
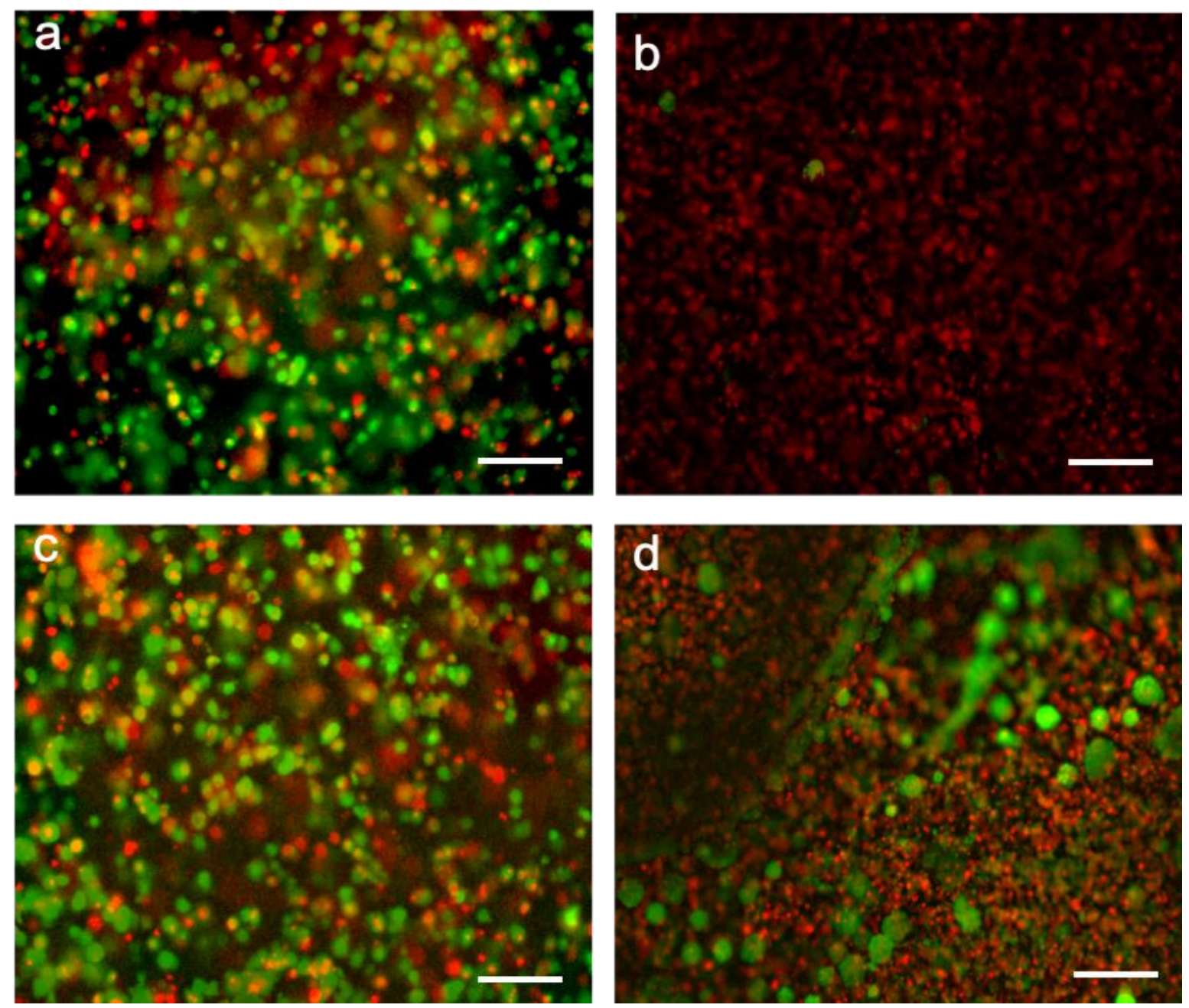

Figure 41. Live/Dead staining of bio-printed LX2 samples at 7 days (a,c) and 13 days $(b, d)$ of cell culture using Cellink bio-ink only $(a, b)$ and ECM+Cellink bio-ink (c,d). Scale bar $200 \mu \mathrm{m}$. 
Alamar blue test carried out after 13 days of cell culture (Figure 41) confirmed that viability and metabolic activity of LX2 cells was statistically significant improved when cells were bio-printed using ECM+Cellink bio-ink compared to Cellink bio-ink.

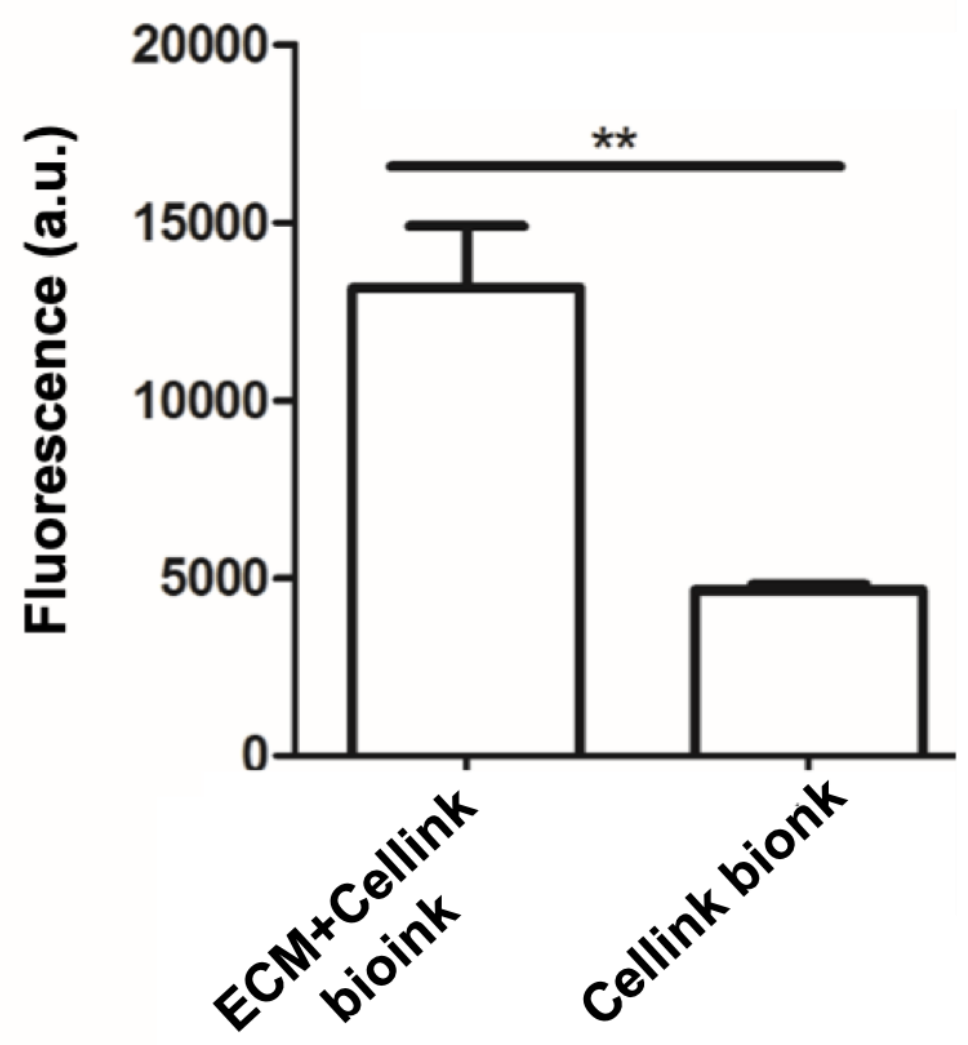

Figure 42. Alamar blue viability test of bio-printed LX2 after 13 days of cell culture. ${ }^{* *} p<0.01$. $\mathrm{n}=4$ per condition.

Haematoxylin and eosin staining (Figure 42) of bio-printed LX2 samples using Cellink bio-ink $(a, b)$ showed the presence of very few alive LX2 organised in single cells. Conversely, LX2 cells bio-printed using ECM+Cellink bio-ink (c,d) were homogeneously distributed into the samples and organised in small spheroids rather than single cells. 

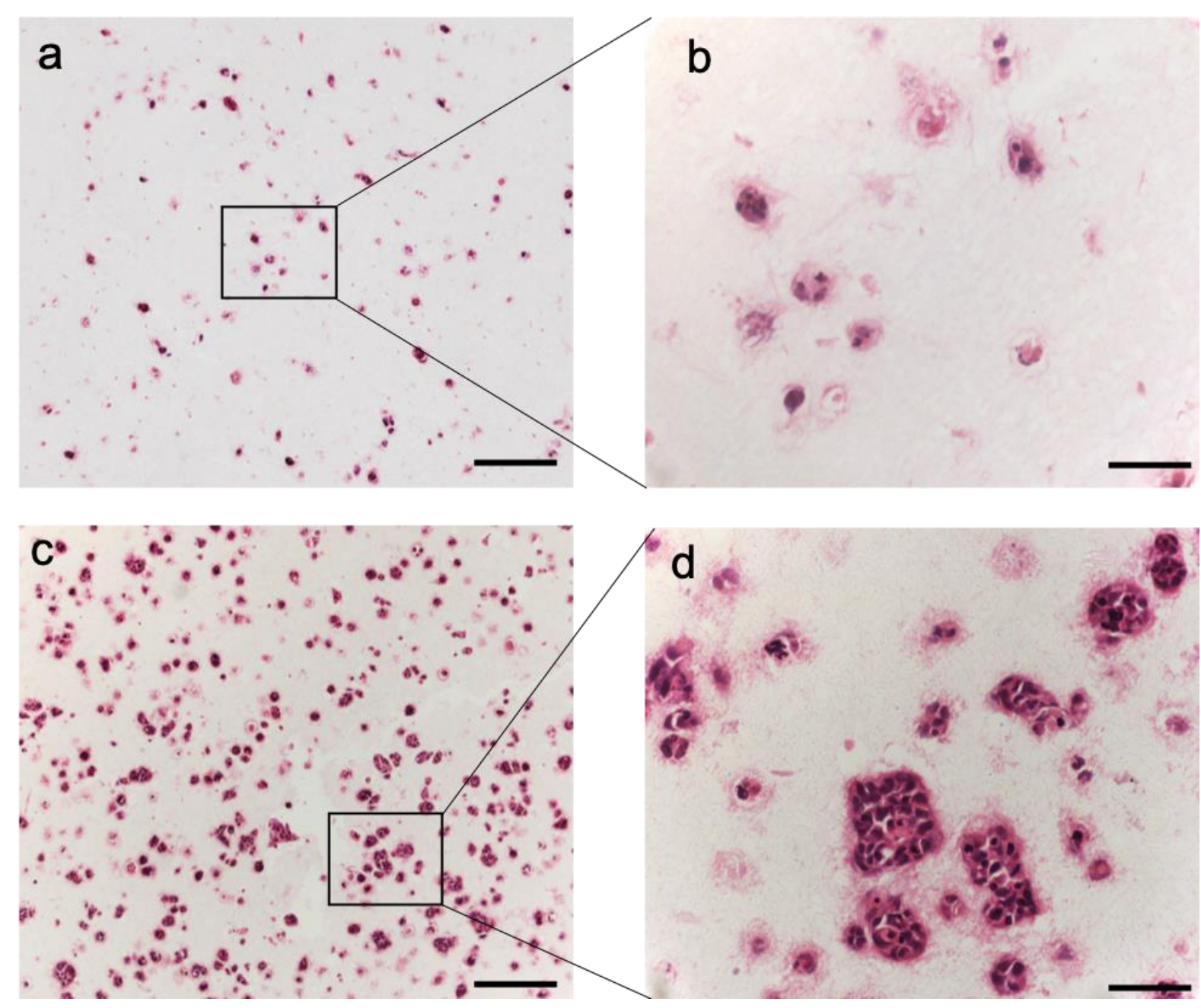

Figure 43. Hematoxylin and eosin staining of bio-printed LX2 samples using Cellink bio-ink $(a, b)$ or $\mathrm{ECM}+$ Cellink bio-ink (c,d) after 13 days of cell culture. Scale bar $200 \mu \mathrm{m}(a, c)$ and 50 $\mu \mathrm{m}(\mathrm{b}, \mathrm{d})$.

RNA was extracted from LX2 bio-printed samples using ECM+Cellink bio-ink Cellink bio-ink. As shown in Figure 43, LX2 mRNA concentration was statistically significant higher in all ECM+Cellink conditions compared to Cellink conditions. Furthermore, the mRNA extracted from ECM+Cellink samples dramatically increased between day 7 and day 14 conditions, while in Cellink bio-ink samples the amount of LX2 mRNA remained approximately the same between day 7 and day 13 conditions. In both LX2 bio-printed samples using Cellink bio-ink or ECM+Cellink bio-ink, TGF- $\beta 1$ treatment caused a decrease in LX2 mRNA extraction. 


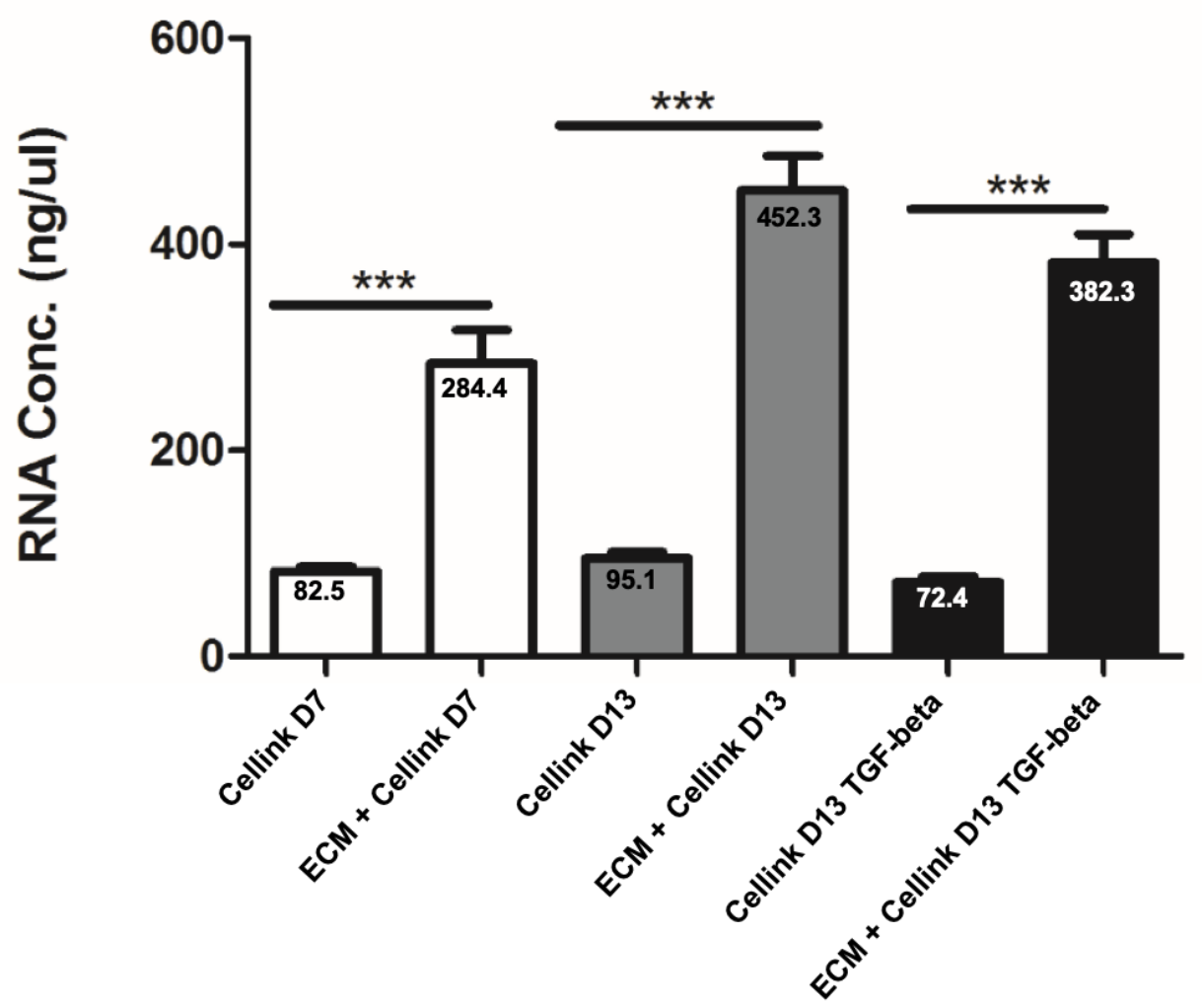

Figure 44. Extracted mRNA from LX2 bio-printed samples after 7 and 13 days of cell culture, with or without exposure to TGF- $\beta 1$ for 6 days. ${ }^{* *} p<0.001$. $n=4$ per condition.

Gene expression analysis of bio-printed LX2 samples (Figure 44) showed that COL1a1 mRNA expression was downregulated in ECM+Cellink samples compared to Cellink bio-ink samples at both 7 and 13 days of cell culture, suggesting a less activated status of LX2. Importantly, this gene was up-regulated upon exposure to TGF- $\beta 1$ for 6 days. ACTA2 was up-regulated at 7 days in ECM-Cellink samples compared to Cellink samples with no differences at prolonged culture conditions even in the presence of TGF- $\beta 1$ treatment. LOX, a liver fibrosis target, was up-regulated in ECM+Cellink samples compared to Cellink samples at both 7 and 13. Important, this gene was only up-regulated upon exposure to TGF- $\beta 1$ for 6 days in ECM+Cellink samples. 

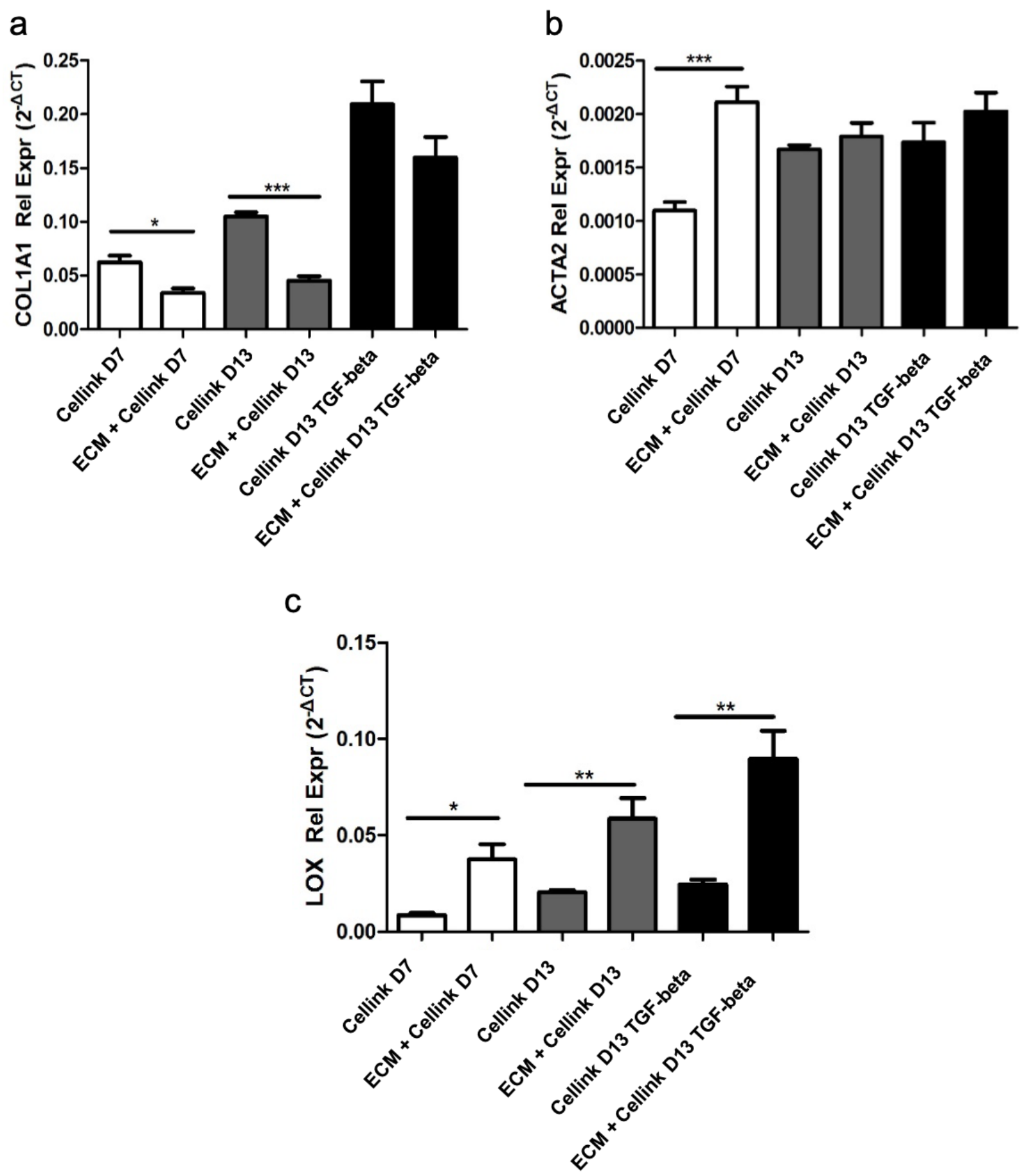

Figure 45. Gene expression of LX2 bio-printed samples at 7 and 13 days of cell culture, with or without exposure to TGF- $\beta 1$ for 6 days. ${ }^{*} p<0.05,{ }^{* *} p<0.01,{ }^{* *} p<0.001 . n=4$ per condition.

Pro-collagen1 ELISA was performed to investigate the pro-fibrotic effect of TGF- $\beta 1$ on LX2 cells. As shown in Figure 45, there was a time dependent statistically significant increase in pro-collagen 1 secretion by LX2 between day 7 and 13 in both ECM+Cellink and Cellink bio-ink. Importantly, LX2 cultured in ECM+Cellink samples were able to 
respond to the pro-fibrotic stimulus of TGF- $\beta 1$, as demonstrated by the increased procollagen1 secretion. Conversely, there was no difference in pro-collagen1 secretion between TGF- $\beta 1$ treated and untreated Cellink bio-ink LX2 samples.

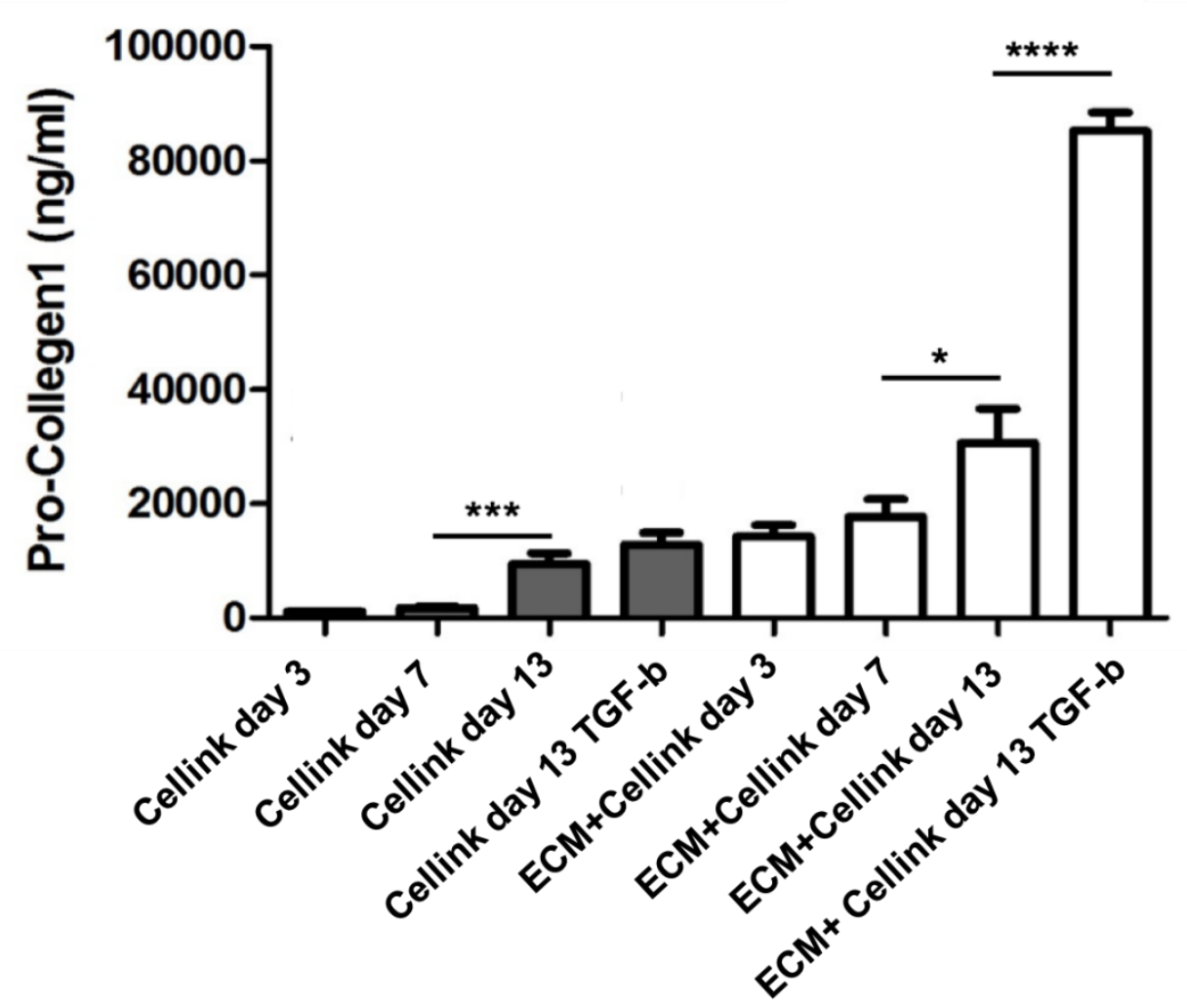

Figure 46. ELISA Pro-Collagen1 secretion from LX2 bio-printed samples at 7 and 13 days of cell culture, with or without exposure to TGF- $\beta 1$ for 6 days. ${ }^{*} p<0.05$, ${ }^{* * *} p<0.001,{ }^{* * * *}$ $\mathrm{p}<0.0001$. $\mathrm{n}=4$ per condition.

\subsubsection{Bio-printing of HepG2 cells}

Chosen sample shape for the bio-printing of HepG2 cells was a cube of $6 \times 6 \mathrm{~mm}$ with a thickness of 2 bio-printed layers. Total amount of HepG2 cells per sample was calculated as 250.000 cells. Bio-printed tissues were maintained in 3D culture up to 13 days and they were analysed by live and dead staining, histology, viability assay, gene expression and Albumin ELISA assay.

Live/Dead staining (Figure 46), of bio-printed HepG2 samples was performed at 7 days $(a, c)$ and 13 days $(b, d)$ of cell culture. After 7 days of cell culture HepG2 bio- 
printed using Cellink bio-ink (a) were found all dead. Conversely, bio-printed HepG2 using ECM+Cellink after 7 days of cell culture (c) showed a higher number of alive cells than dead cells. After 13 days of cell culture (d) HepG2 cells were found mainly alive and organised in spheroids.
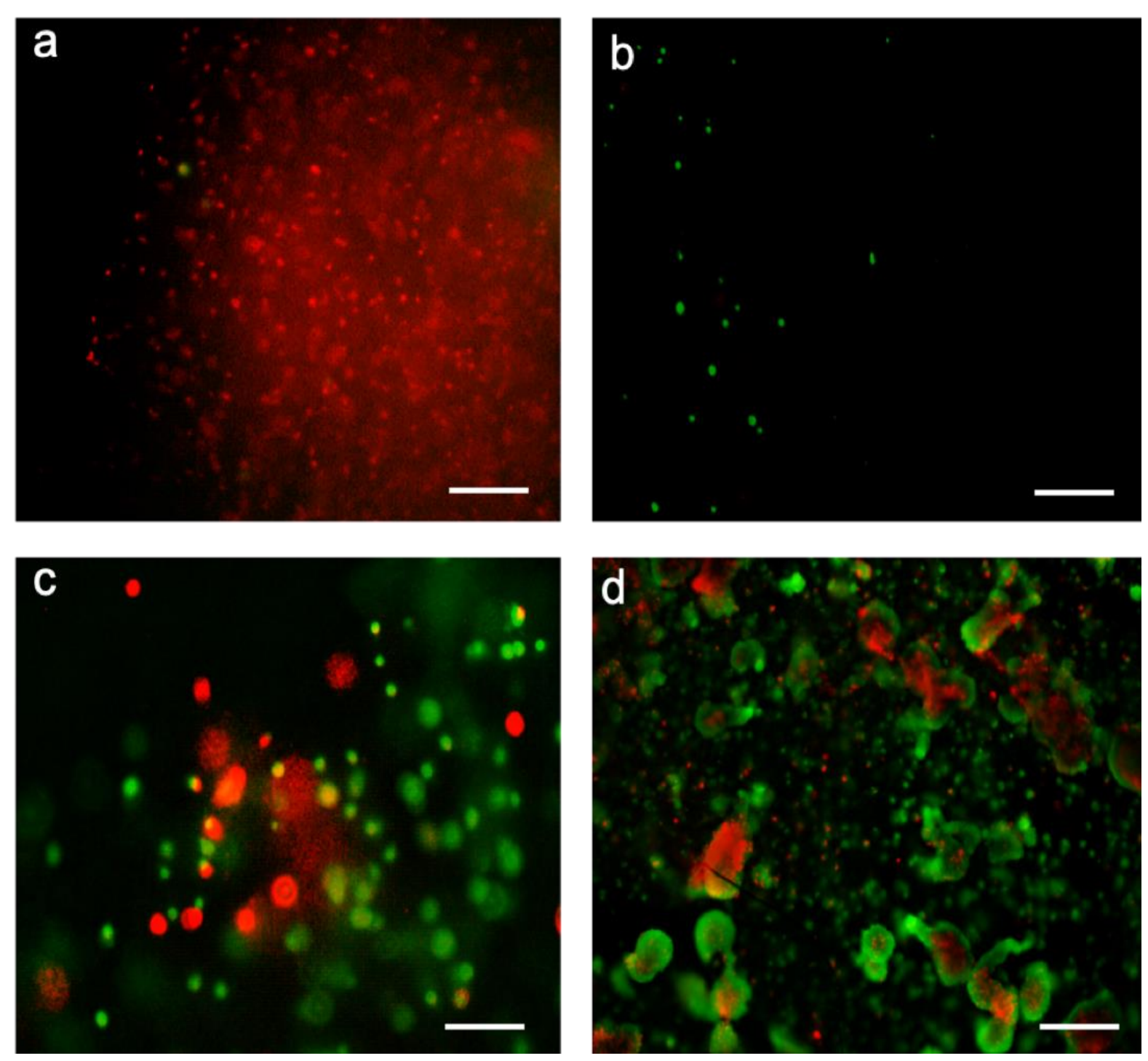

Figure 47. Live/Dead staining of bio-printed HepG2 samples at 7 days (a,c) and 13 days (b,d) of cell culture using Cellink bio-ink (a,b) and ECM+Cellink bio-ink (c,d). Scale bar $200 \mu \mathrm{m}$.

Alamar blue test performed after 13 days of cell culture (Figure 47) confirmed that viability and metabolic activity of HepG2 cells was statistically significant higher in $\mathrm{ECM}+$ Cellink samples compared to Cellink samples, which generated the same fluorescence signal as empty scaffolds, confirming that all HepG2 cells were dead. 


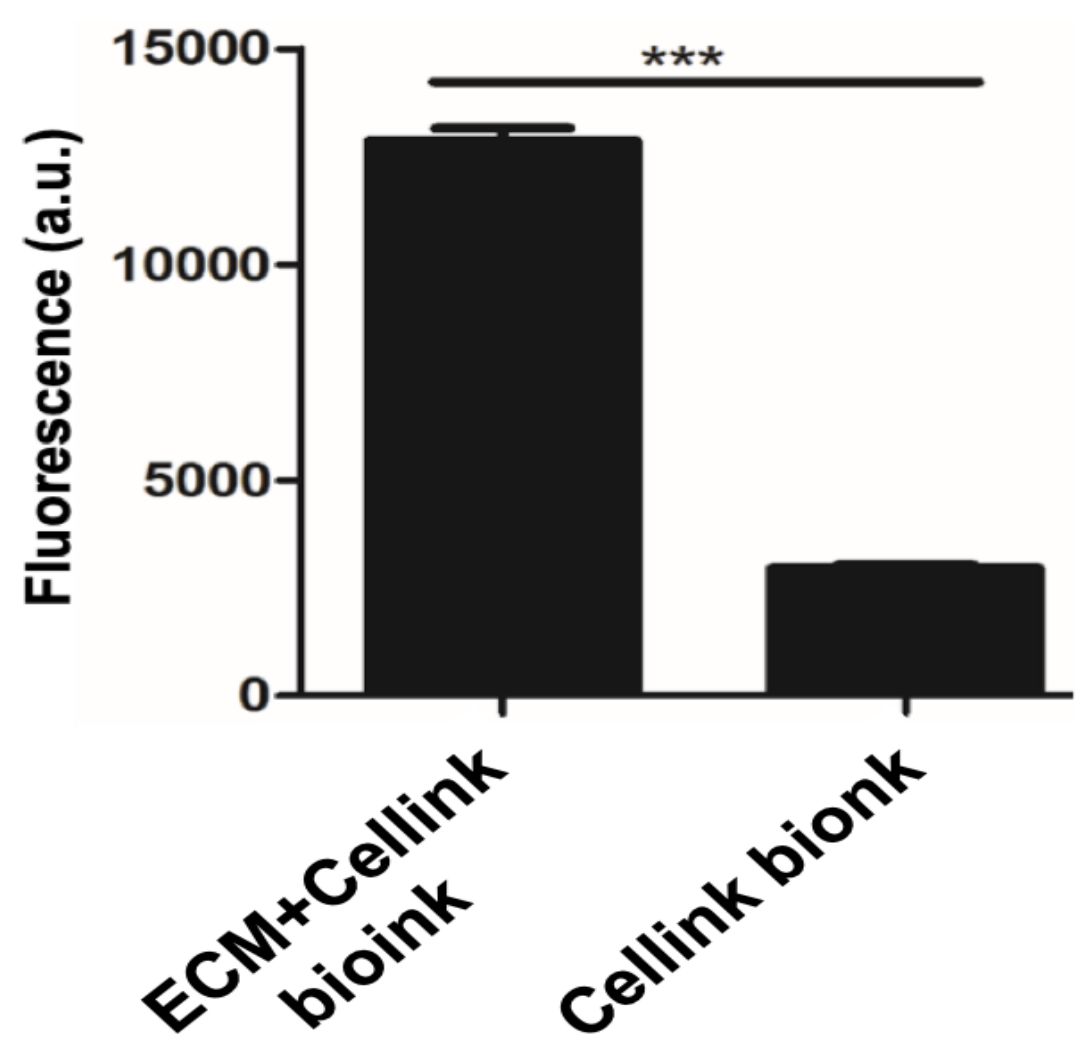

Figure 48. Alamar blue viability test of bio-printed HepG2 samples after 13 days of cell culture ${ }^{* * *} \mathrm{p}<0.001$. $\mathrm{n}=4$ per condition.

Haematoxylin and eosin staining (Figure 48) of bio-printed HepG2 samples using Cellink bio-ink $(a, b)$ showed the presence of very few HepG2 cells and probably not alive since the haematoxylin staining for the nuclei was not present. On the other hand, HepG2 cells in ECM+Cellink samples (c,d) were alive, homogeneously distributed into the samples and cells were organised in spheroids of around $200 \mu \mathrm{m}$. 

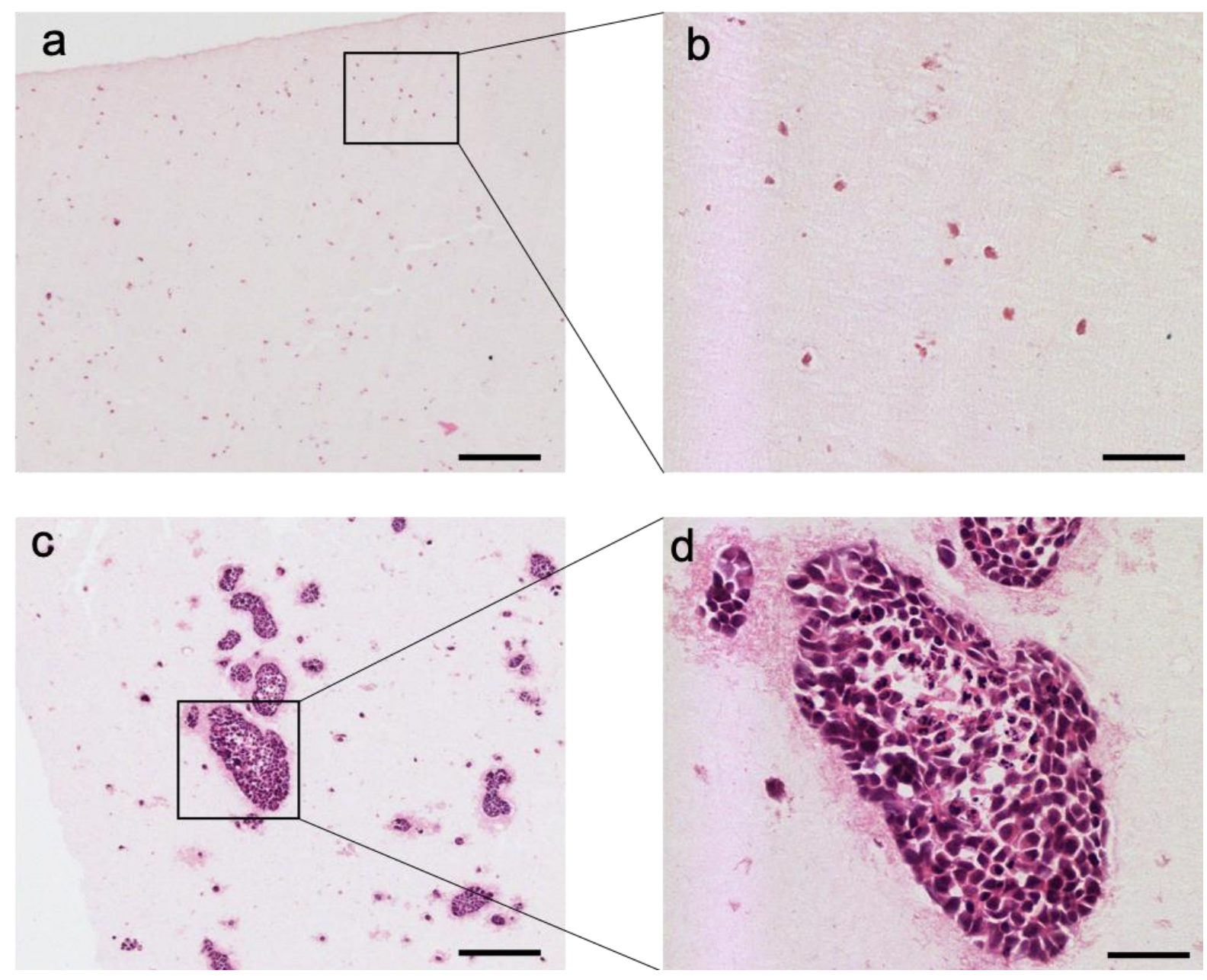

Figure 49. Hematoxylin and eosin staining of bio-printed HepG2 cells using Cellink bio-ink $(a, b)$ or ECM+Cellink bio-ink (c,d) after 13 days of cell culture. Scale bar $200 \mu \mathrm{m}(a, c)$ and 50 $\mu \mathrm{m}(\mathrm{b}, \mathrm{d})$.

Figure 49 illustrate the mRNA amount extracted from HepG2 bio-printeded samples using Cellink bio-ink or ECM+Cellink bio-ink. As shown in this graph, HepG2 mRNA concentration was statistically significant higher in all ECM+Cellink conditions compare to Cellink bio-ink conditions. Concentration of mRNA extracted from HepG2 bio-printeded samples using Cellink bio-ink was too low to be reliably used for further gene expression analysis. 


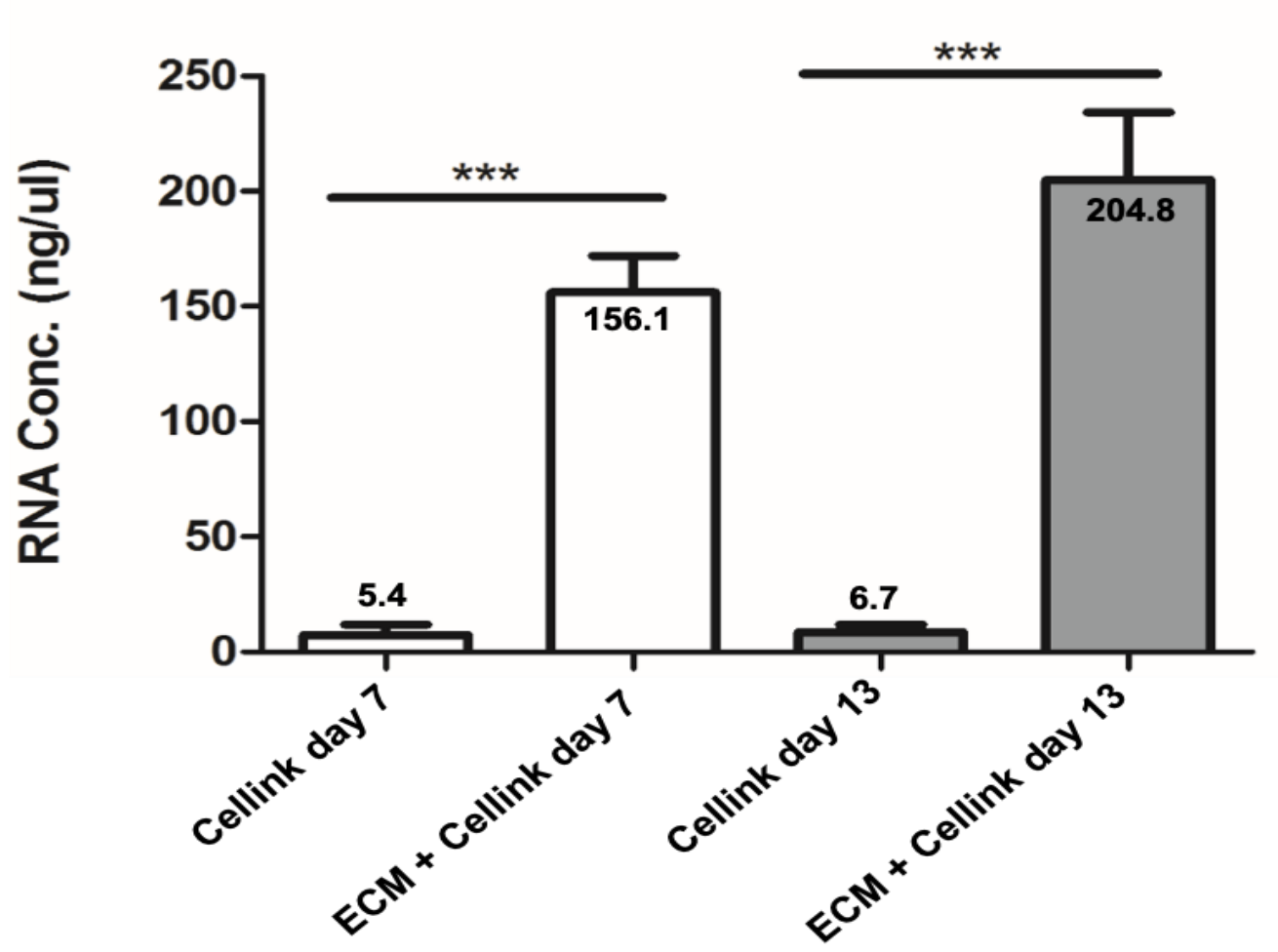

Figure 50. Extracted mRNA from HepG2 bio-printeded samples after 7 and 13 days of cell culture. ${ }^{* *} p<0.001$. $n=4$ per condition.

Gene expression analysis (Figure 50) was performed only on HepG2 bio-printeded using ECM+Cellink bio-ink since a not adequate amount of RNA was extracted from Cellink samples. Gene expression showed a time-dependent statistically significant increase in the mRNA expression of Albumin (a), as well as AFP (c). mRNA levels of HNF4A (b) did not statistically significant change between day 7 and day 13 of cell culture. 

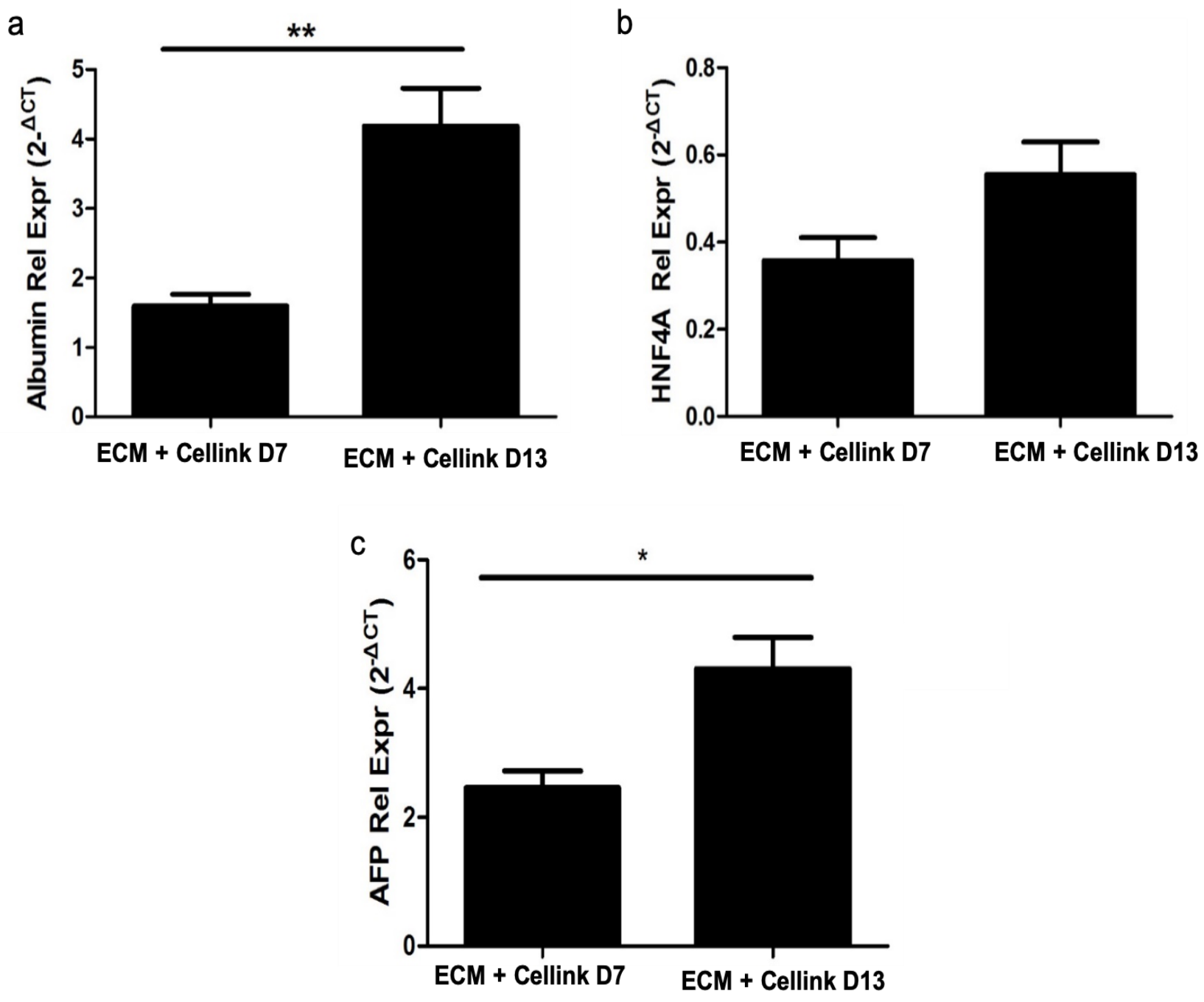

Figure 51. Albumin, HNF4A and AFP mRNA expression of HepG2 in ECM+Cellink bioprinteded samples at 7 and 13 days of cell culture. ${ }^{*} p<0.05,{ }^{* *} p<0.01 . n=4$ per condition.

HepG2 Albumin secretion (Figure 51) was analysed only in ECM+Cellink samples because HepG2 did not survive in Cellink bio-ink samples. Resulting graph showed a time-dependent statistically significant increase in secreted albumin from ECM-Cellink samples between day 3, 7 and 13 of cell culture, confirming the high metabolic activity of HepG2 cells in the presence of the liver ECM proteins. 


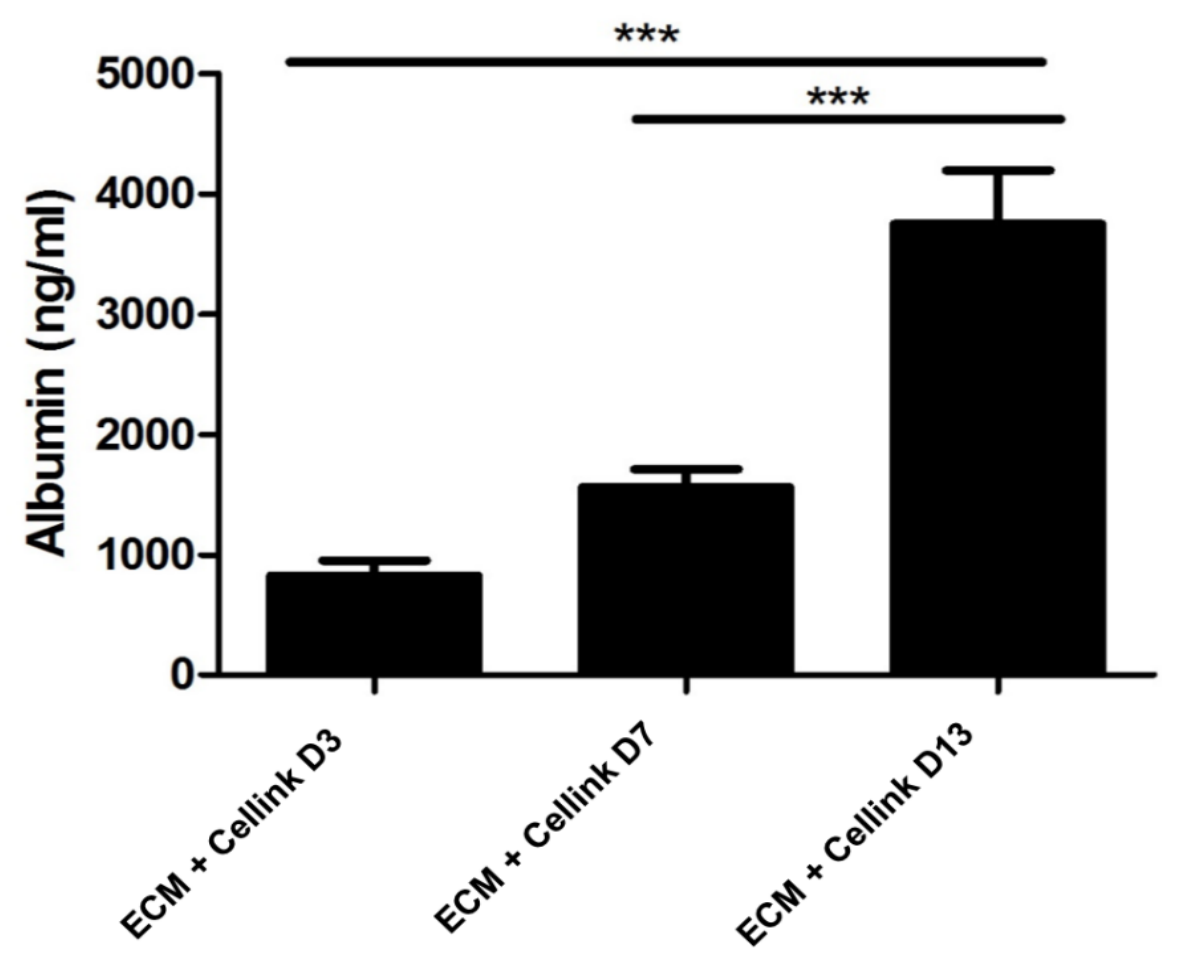

Figure 52. ELISA Albumin secretion from HepG2 bio-printed samples at 3, 7 and 13 days of cell culture. ${ }^{* * *} p<0.001 . n=4$ per condition.

\subsubsection{Bio-printing of primary human hepatocytes}

Chosen sample shape for the bio-printing of primary human hepatocytes cells was a cube of $4 \times 4 \mathrm{~mm}$ with a thickness of 2 bio-printed layers. Total amount of human primary hepatocytes per sample was calculated as 400.000 . Bio-printed tissues were maintained in 3D culture up to 10 days and they were analysed by Live/Dead staining, albumin and lactate dehydrogenase (LDH) ELISA assays.

Live/Dead staining (Figure 52), of bio-printed primary human hepatocytes using $\mathrm{ECM}+$ Cellink bio-ink was performed at 10 days of cell culture. Resulting image showed that primary human hepatocytes were found all alive after 10 days of cell culture. 


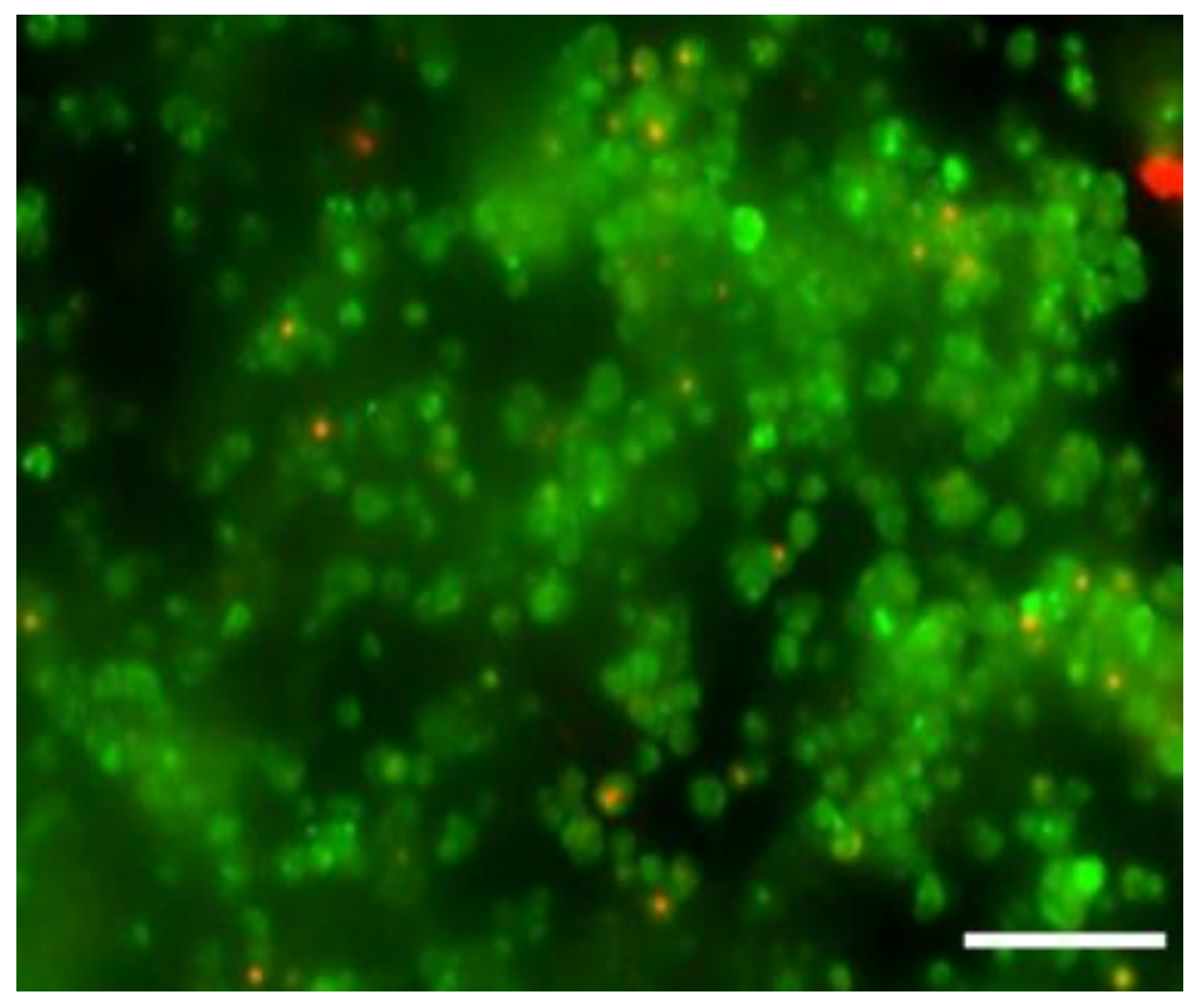

Figure 53. Live/Dead staining of bio-printeded primary human hepatocytes samples at 10 days of cell culture using ECM+Cellink bio-ink. Scale bar $200 \mu \mathrm{m}$.

Primary human hepatocytes cultured in ECM+Cellink samples showed that lactate dehydrogenase (LDH) (Figure 53 a) was strongly increased during the first days of cell culture followed by a statically significant decrease in LDH secretion from day 5 of cell culture. At the same time albumin secretion (Figure 53 b) showed a time-dependent statistically significant increase during the cell culture, suggesting that the cells require an adaption time to reactivate their normal metabolic activity. 

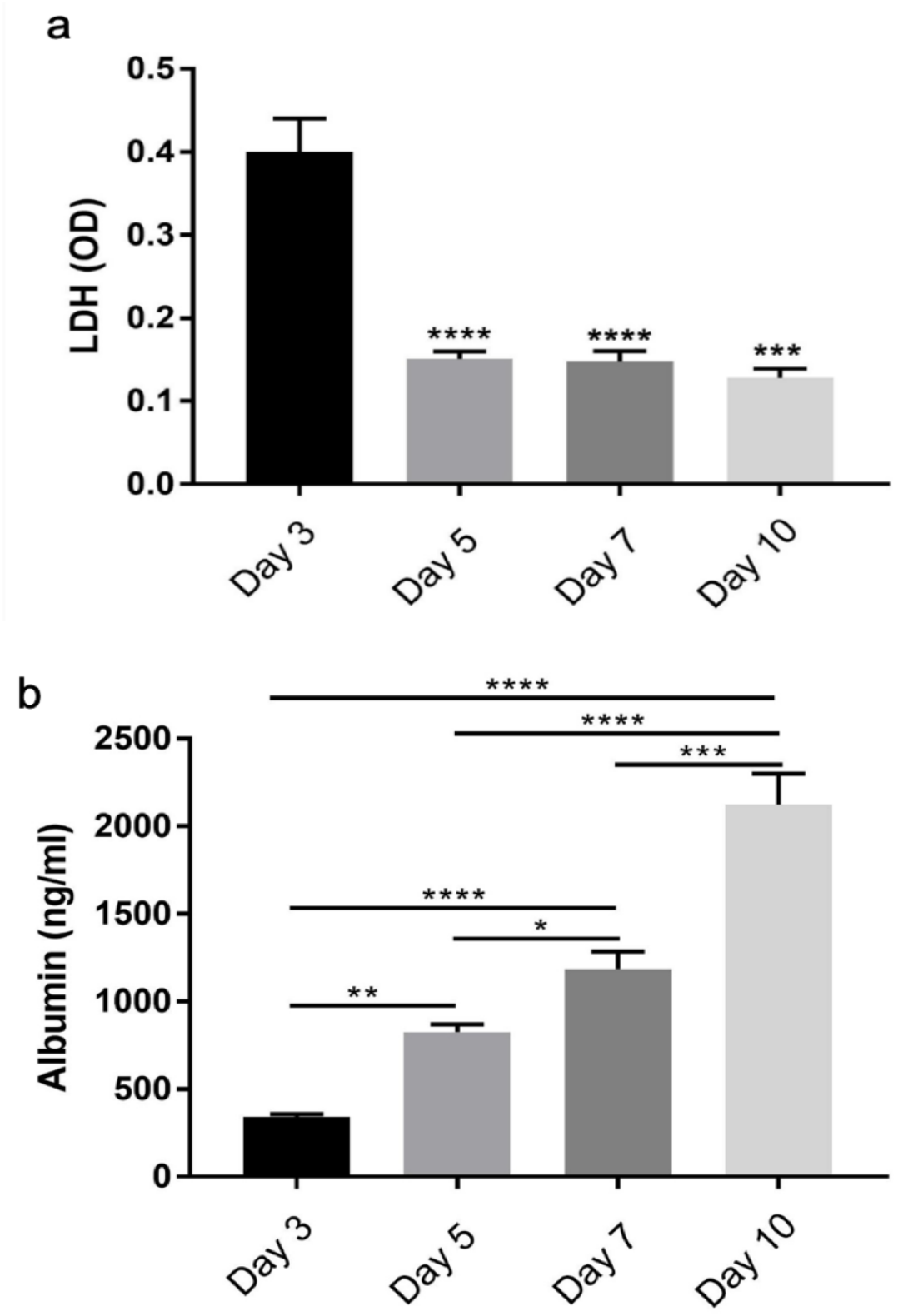

Figure 54. ELISA of (a) Lactate Dehydrogenase secretion and (b) Albumin secretion from primary human hepatocytes samples at $3,5,7$ and 10 days of cell culture. ${ }^{*} p<0.05,{ }^{* *} p<0.01$, ${ }^{* * *} p<0.001,{ }^{* * *} p<0.0001$. $n=4$ per condition. 


\subsection{Discussion}

\subsubsection{Advantages of ECM+Cellink bio-ink compared to available bio-inks}

In the last decades, 3D cell printing has become a helpful technology in the field of tissue engineering and regenerative medicine as it allows the accurate deposition of multiple cells onto a pre-defined position, moving a step forward to recapitulate the microenvironment of native tissue [79].

Different natural and synthetic biomaterial, alone or in combination, have been used as bio-ink for 3D cell printing. Several biomaterials used as bio-inks for 3D printing include agarose, collagen, cellulose, hyaluronic acid, PEG and PLC. With the exception of collagen and hyaluronic acid, all these biopolymers are characterised by excellent mechanical and printability properties [78]. However, they do not contain tissue specific bioactive cues thus are not able to mimic the microenvironment of native tissues and organs that is essential to support cellular adhesion, viability and activity [80]. Therefore, decellularized extracellular matrix is the optimal source of bioink because of its unique advantage of preserving the bioactive cues and the appropriate microenvironment of the native organs and tissue to promote cell engraftment, survival, and function [282].

Several studies have reported the feasibility of using bio-inks composed of decellularized extracellular matrix. In the first study, Pati et al. used porcine heart, adipose tissue and cartilage as source of ECM [278]. Although the author showed improvement in metabolic function of bio-printeded tissue in the presence of ECM, the extracellular matrix derived from xenogeneic origin could limit the translation towards Good Manufacturing Practices (GMP) and clinical applications. Furthermore, to enhance the printability of the ECM solutions, Poly-caprolactone (PLC) bio-inks were used to create a solid framework. PLC bio-ink is not suitable for cell printing, since it requires elevated temperatures and high extrusion pressure. The authors used nonphysiological bio-printing conditions as PCL was loaded in a syringe and heated to $80^{\circ} \mathrm{C}$ to melt the polymer. The applied pneumatic pressure was in the range of 400 $650 \mathrm{kPa}$ for fabrication of the PCL framework. At the same time, the decellularized 
ECM bio-inks mixed with the cells were loaded to the other syringe and maintained at temperatures below $15^{\circ} \mathrm{C}$.

In 2017, Lee et al. described the employment of porcine liver decellularized extracellular matrix bio-ink, in combination with PLC bio-ink, for 3D cell printing-based liver tissue engineering [80]. In this study the authors showed a better stem cell differentiation and HepG2 cell functions in the liver dECM bio-ink constructs compared to collagen bio-ink constructs. Again, non-physiological conditions were used for bioprinting and $\mathrm{PCL}$ was printed at a temperature of $60{ }^{\circ} \mathrm{C}$ and a pneumatic pressure of $500 \mathrm{kPa}$ was used, while porcine liver ECM was mixed with the cells and printed at $15^{\circ} \mathrm{C}$ under pneumatic pressures in the range of $5-60 \mathrm{KPa}$. Shear stress can have a negative impact on cell viability and indeed shear stress above $10 \mathrm{kPa}$ demonstrated to have an adverse effect on human mesenchymal stem cell and L929 mouse fibroblast viability [283].

One limitation is that the authors used decellularized ECM derived from porcine liver. As mentioned in the discussion of the previous Chapter, the healthy porcine liver ECM is characterised by well-defined lobules outlined by connective tissue, which is absent in healthy human liver and only present in fibrotic human liver [213, 284]. Therefore, human organs are the most suitable source of ECM to mimic the human physiological microenvironment for the in vitro culture of human cells and for clinical applications [285, 286].

In this project we evaluated the possibility of using human liver ECM as bio-ink for 3D bio-printing in order to develop tissue specific constructs to be used in drug discovery of hepatic diseases and for drug toxicity testing. Human liver ECM solution was combined with Cellink bio-ink, a nanocellulose based bio-ink containing a small percentage $(0.5 \%)$ of sodium alginate, to enhance the mechanical properties and therefore the printability property. Compared to the previously described bio-inks, the resulting $\mathrm{ECM}+$ Cellink bio-ink has several advantages such as 1) the possibility to bioprint under physiological conditions, such as room temperature and extrusion pressure below $10 \mathrm{KPa}, 2)$ reducing the negative impact on cell viability caused by shear stress. Another key advantage of ECM+Cellink bio-ink is the unique tissue specific composition, thus providing a new in vitro model composed of human liver ECM components. This advantage in unique tissue specific composition should provide the 
essential cues to mimic the liver microenvironment and to allow the hepatic cells to behave in vivo like [213].

\subsubsection{Printability tests using ECM+Cellink bio-ink}

The printability tests confirmed the feasibility of printing human liver ECM in solution together with CELLINK bio-ink at different ratios ECM:Cellink, respectively 50:50, 30:70 and 20:80. As shown in this chapter, different sizes of sample cubes were bioprinted and recorded. This was done to reduce the volume of ECM solution needed while increasing the ratio ECM:Cellink from 20:80 to 50:50. The best results in terms of spatial accuracy of bio-ink deposition was obtained by the bio-inks in ratios ECM:Cellink 20:80 and 30:70. However, although the bio-ink composed by a ratio ECM:Cellink 50:50 showed to be not suitable for the printing of complex shapes such as a well-defined spiral, it demonstrated to be suitable for the bio-printing of cube samples. Important, the 50:50 ECM:Cellink composition, compared to the other ratios, was characterised by superior bioactive properties and can be bio-printeded using a lower extrusion pressure, reducing the negative effect of shear stress on cell viability and behaviour. Considering all these aspects, the bio-ink composed by ECM solution and Cellink bio-ink in a ratio 50:50 was chosen for the bio-printing of liver tissue specific constructs described in this Chapter.

\subsubsection{In vitro biocompatibility of ECM+Cellink bio-ink}

The in vitro biocompatibility of bio-printeded ECM+Cellink or Cellink samples, the latter employed as a control, was first investigated using the cell lines LX2 and HepG2 and this in order to reduce the variability intrinsic with the use of primary cells derived from different donors, such as primary hepatocytes and hepatic stellate cells [287].

For both LX2 and HepG2 hepatic cell lines, ECM+Cellink bio-ink, showed better bioactive properties to promote cell engraftment, survival, and function compared to Cellink bio-ink only used as control. 
To validate new in vitro models it is necessary to demonstrate cell viability in the new substrate. As confirmed by Live/Dead staining and Alamar Blue viability test, an advantage of using the combination of human liver ECM+Cellink showed that LX2 cells had a much higher viability than bio-printed cells with Cellink bio-ink further indicating a pro-survival potential of the human ECM liver component in the system. Nevertheless, a Live/Dead staining and Alamar Blue performed at 24 hours from bioprinting would have been useful to evaluate the direct effect of shear stress on cell viability after this process.

LX2 gene expression analysis showed that COL1a1 is downregulated in ECM+Cellink samples compared to Cellink bio-ink samples, suggesting a less activated status of hepatic stellate cells when grown in human ECM. This condition is required for the development of an efficient in vitro fibrosis model [288, 289]. LOX, a liver fibrosis target, is an enzyme involved in the cross-linking of collagen and elastin [290] and indeed it is statistically significant expressed more in ECM+Cellink samples compared to Cellink samples, suggesting the key role of ECM in modulating also the expression of this enzyme. Moreover, upon exposure to TGF- $\beta 1$ LOX mRNA expression is upregulated, confirming that LX2 cells in this new 3D model are sensitive/responsive to the pro-fibrotic stimulus.

Pro-collagen 1 is abundantly secreted during liver fibrosis progression and one of the TGF- $\beta 1$ key function is to activate stellate cells and increase the expression and secretion of collagen 1 [291]. Indeed, LX2 cells grown in ECM+Cellink and exposed to TGF- $\beta 1(5 \mathrm{ng} / \mathrm{ml})$ for 6 days secreted dramatically higher level of pro-collagen1 compared to untreated samples at day 13. This difference in secretion of pro-collagen was not observed between treated and untreated Cellink samples, confirming the importance of adding the human liver ECM to develop an efficient 3D model for the in vitro study of liver fibrosis.

The importance of ECM proteins for cell adhesion, viability, proliferation and functionality was further confirmed by culturing HepG2 cells. Indeed, in Cellink bioprinteded samples HepG2 were found all dead at 7 days whereas HepG2 cells grown in ECM+Cellink were mainly alive. Similar to LX-2 samples, a Live/Dead staining and Alamar Blue performed at day 1 of cell culture would have been useful to evaluate the effect of shear stress on HepG2 cell viability after the bio-printing process. Both gene 
expression analysis and albumin ELISA showed a time-dependent increased expression of Albumin, suggesting a stronger metabolic-like phenotype, as well as AFP expression, which is a hepatoblastoma marker linked to proliferation.

Considering how the ECM microenvironment positively influences cell viability and functionality, bio-printing of primary human hepatocytes was performed using $E C M+$ Cellink bio-ink. Live and dead staining confirmed that primary human hepatocytes were still alive after 10 days of cell culture. The trend of lactate dehydrogenase (LDH) and albumin secretion during the cell culture was similar to that of primary human hepatocytes cultured in human 3D liver scaffolds [154]. Lactate dehydrogenase (LDH) was strongly increased during the first days of repopulation to decrease and being not detectable and this trend was in parallel with a significant increase in albumin and factor IX secretion. This indicates that the primary human hepatocytes require a certain time to adapt to this 3D printed construct.

Although albumin and LDH analysis confirmed the feasibility of bio-printing primary hepatocytes bio-printed using ECM+Cellink bio-ink, a better characterisation of these samples should have been done. For example, would have been interesting to evaluate the expression of important hepatic genes such as albumin and CYPs to investigate the metabolic activity of the cells, CCAAT-enhancer-binding proteins (C/EBP $\alpha$ and $\beta$ ) to investigate the hepatocytes proliferation, AAT, transferrin, and the gene levels of the enzymes involved in the urea cycle including OTC and CPS1.

\subsubsection{ECM+Cellink bio-ink vs ECM-Agarose hydrogels}

Compared to the ECM-Agarose hydrogel described in the previous Chapter, bioprinting using ECM bio-ink presents some important advantages, in particular the possibility to mix the cells with ECM before the bio-printing. Indeed, when using the ECM-Agarose hydrogels it was not possible to mix the cells with the ECM-Agarose pre-gel solution because of its fast gelation time and for this reason cells were seeded on top of the ECM-Agarose hydrogels. Mixing the cells before bio-printing is an important advantage as it allows to decrease the total cell number needed and to reduce the cell number variability between each bio-printeded sample. When cells were seeded on top of the ECM-Agarose hydrogels, only a fraction of the cells 
attached to hydrogels, while the rest of the cells adhered to the plastic of the culture well plate. As a consequence, this did not allow to know the exact cell number per ECM-Agarose hydrogel. This further affected the standardisation of the results. Using the 3D bio-printing technique, the cells are already mixed with the ECM solution and homogeneously distributed inside the ECM+Cellink samples. This allowed to reduce the number of cells needed in comparison to the ECM-Agarose hydrogels, more specifically from 0.5 to 0.25 million in each sample when using cell lines and from 0.5 to 0.4 million when repopulating primary human hepatocytes. Thus, ECM plus Cellink bio-printing favours a consistent total cell number per bio-printeded sample which reduces intervariability and induces consistency between samples as confirmed by the low standard deviation in the outcomes as shown for gene expression.

Another important advantage of the 3D bio-printing technique is the possibility to perform co-cultures of hepatic cells to maintain the correct in-vivo like cell distribution. Indeed, considering that hepatocytes and stellate cells account respectively for approximately $60 \%$ [292] and 5-8\% [293] of the total cells in the human liver, it would be possible to bio-print these two liver cell types in a ratio of hepatocyte: stellate cells 9:1 and this to maintain their in vivo ratio. As previously discussed, when using the ECM-Agarose hydrogels to grow co-cultures and to maintain this in vivo like cell ratio, both cell types needed to be seeded on top of the gels, which induced greater variability in cell attachment.

On the other hand, 3D bio-printing presents also some limitations such as the usage of calcium chloride as cross-linking. Calcium ions, with a cellular concentration of $2 \mathrm{mM}$, are involved in several cell functions including enzyme activities, motility, metabolic processes, signal transduction, proliferation and apoptosis [294]. Cao et al. demonstrated that $100 \mathrm{mM}$ calcium chloride solution, employed as crosslinking agent for the preparation of alginate hydrogels, caused a significant reduction in living cells due to the cell damage caused by the high calcium concentration [76].

Moreover, 3D bio-printing is characterised by several technical difficulties. Indeed, the $3 \mathrm{D}$ printer must be placed inside the laminar flow limiting the working space and hampering the sterility. Furthermore, several mixing steps are required to obtain a homogeneous bio-ink and this causes a loss of the biomaterial during the process. All 
together these limitations hamper the applicability of bio-printing process for high throughput screening (HTS) technology.

Overall the current study demonstrated the feasibility of employing human liver ECM solution for 3D bio-printing of tissue specific constructs that can be used for in vitro drug discovery of hepatic diseases and for drug toxicity testing. However, the high number of cells needed to obtain a functional in vitro platform, negatively affects their performance in high throughput screening (HTS) of new drugs.

\subsubsection{Bio-printing applications}

Despite the enormous technological improvements occurred in this field, bio-printers still do not allow us to fabricate a solid organ for transplantation. The biggest limitations include the printing time needed to develop a functional organ and the lack of required resolution to print the vasculature network [295, 296]. To date, this technology allows us to create structures with a resolution of $100-200 \mu m$ suitable for medium blood vessel, but it is inadequate for replicating capillaries of $5-10 \mu \mathrm{m}$ [297]. New improvements in this field are required to overcome these limitations in order to fabricate functional solid organs and increase the pool of available organs for transplantation. On the contrary, decellularization-recellularization allows us to preserve the natural 3D architecture as well as the vasculature network of the native organs, offering a better chance of developing a transplantable organ [87].

On the other hand, bio-printing can be applied to develop in-vitro models for target validation and drug screening.

This technology can also be applied to fabricate implantable liver tissue for the treatment of metabolic liver disorders in paediatric population. In this context Dhawan's team [298] directly infused primary human hepatocytes (pHep) via the portal vein at a dose of $10^{8} \mathrm{pHep} / \mathrm{Kg}$ of human body weight. Therefore, the number of cells needed for a $10 \mathrm{~kg}$ paediatric patients is $10^{9}$ Hepatocytes.

However, this approach failed to show long-term clinical benefits due to poor cell engraftment. Indeed, it is known that $\sim 1 \%$ of the cells infused will engraft in the liver 
so the real therapeutic dose should be adjusted to $10^{7}$ primary human hepatocytes. Most likely 10 millions of cells will not be enough to treat metabolic disorders therefore a therapeutic dose range for implantable liver tissue should be fully addressed in order to evaluate restoration of metabolic function.

A possible strategy to overcome these limitations is to bio-print primary human hepatocytes using ECM-Cellink bio-ink. The current bio-printed tissue employs a cell concentration of $10 \times 10^{6} / \mathrm{ml}$ with the possibility of printing $4 \mathrm{~mm} \times 4 \mathrm{~mm} \times 1 \mathrm{~mm}\left(16 \mathrm{~mm}^{3}\right)$ of tissue with $1 \times 10^{6}$ cells. According to this cell concentration, $65 \mathrm{~mm} \times 65 \mathrm{~mm} \times 4 \mathrm{~mm}$ $\left(>16000 \mathrm{~mm}^{3}\right)$ of tissue will be needed to implant 1 billion primary human hepatocytes and obtain $5 \%$ of liver mass. In the human body, the maximum distance between the cells and blood vessel for the optimal nutrients and oxygen uptake is approximately $200 \mu \mathrm{m}$ [299]. For this reason, the thickness of implantable liver should be as small as possible in order to avoid cell death and necrosis. Moreover, the possibility of fabricating implantable liver tissues having defined pores should improve the diffusion of oxygen and nutrients to the cells.

Further, an alternative possibility is to use the power of proliferation of hepatic stem cells to multiply and generate a viable number of hepatocytes in the model, thus reducing the size of the implantable liver tissue and the number of cells needed to restore metabolic functions.

Considering the elevated vascularization and the extended surface of the omentum up to $1500 \mathrm{~cm}^{2}$ [221], this organ is an ideal place to implant the bio-printed liver tissues. The $4 \mathrm{~mm} \times 4 \mathrm{~mm} \times 1 \mathrm{~mm}$ tissue size with $1 \times 10^{6}$ tissue will be the maximum therapeutic dose to be used in mice pre-clinical models. 


\section{Chapter 4: Hep-Gel, a novel in vitro 3D model for the study of Hepatocellular carcinoma (HCC) and drug screening tests}

\subsection{Introduction}

This Chapter addresses the development of a thermoresponsive hydrogel using human liver ECM solution in combination with a synthetic thermoresponsive copolymer (2-hydroxypropylmethacrylate and Poly-glycerolmonomethacrylate) kindly provided by Professor Giuseppe Battaglia, UCL Chemistry department. The thermoresponsive copolymer/liver ECM (Hep-Gel) suitability will be tested for high throughput screening (HTS) of new drugs for the treatment of liver diseases and for assessing drugs-related hepatotoxicity. Data reported in this Chapter have been incorporated into a patent (UK patent application No. 1904793.5).

\subsubsection{Hepatocellular carcinoma (HCC)}

The progress in health and technology fields in the last decades have reduced the mortality in most of the diseases with the exception of liver disease. Indeed, since 1970, the incidence of liver disease increased more than 400\% [300, 301] (Figure 54). Hepatocellular Carcinoma ( $\mathrm{HCC}$ ) is considered the most common liver disease among all liver malignancies, and accounts for $90 \%$ of all liver cancer cases in the world [302]. Moreover, in 2016, Hepatocellular Carcinoma was considered the fifth cause of cancer dead worldwide with approximately 800,000 deaths. In the same year, almost 5,500 deaths caused by HCC have been reported in the UK [303].

Regarding the HCC epidemiology, men are more likely to develop HCC compared to women (2.4:1 proportion) [304]. The highest incidence of this pathology has been registered in the Eastern Asia and Western Africa population with a rate above 20 cases every 100,000 individuals. The HCC incidence rate in the European population 
is $10-15$ cases every 100,000 individuals while in the case of the North and South American population this is rated below 5 cases every 100,000 individuals [305].

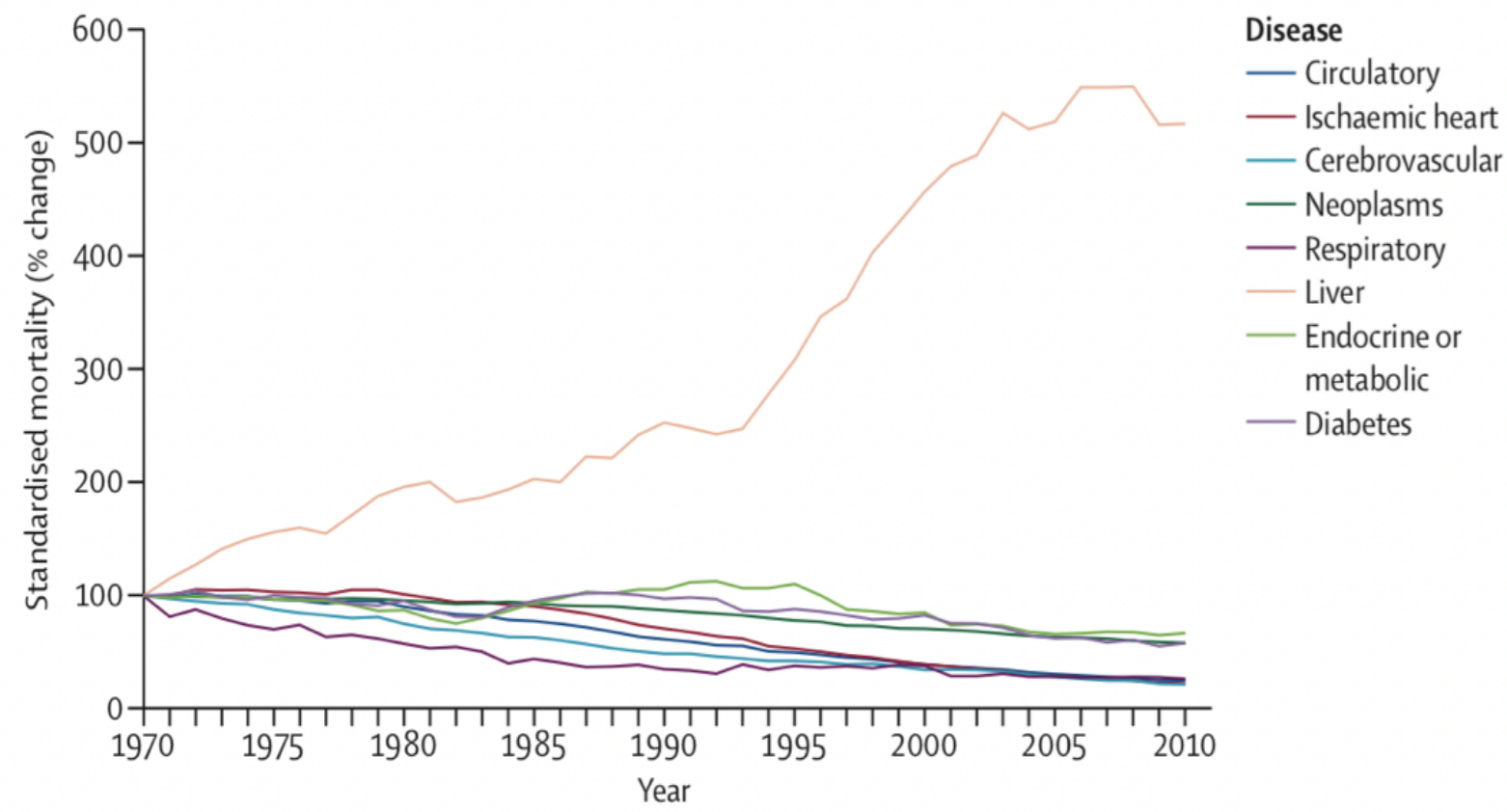

Figure 55. UK mortality rate data since 1970. Graphic retrieved from R. Williams et al. [300]. This graph was also used in the 2009 National Plan for Liver Services UK [306].

Subjects affected by Chronic Liver Diseases (CLD) such as Hepatitis B virus infection (HBV), Hepatitis C virus infection (HCV), Alcohol-related liver disease, Diabetes and Non-Alcoholic Steatohepatitis (NASH) are the one with higher risk to develop HCC [307]. These pathologies cause changes and mutations in the hepatocytes, which become more prone to develop a neoplastic behaviour. Moreover, the stromal cells, particularly the Hepatic Stellate Cells (HSC) [308] become activated, increasing the ECM deposition throughout the organ. Liver fibrosis is characterised by abnormal deposition of Extracellular Matrix (ECM) in the parenchyma and can ultimately lead to liver cirrhosis. Important, it has been shown that $90 \%$ of HCC cases is developed in fibrotic or cirrhotic subjects [309] with a survival rate of $50 \%$ after 2 years from the diagnosis and only $10 \%$ after 5 years [310]. 


\subsubsection{Factors promoting HCC}

The low efficiency of the current HCC treatments and the consequent high mortality rate of this disease are related to its heterogeneity [311]. Indeed, HCC is a multiple steps disease in which each phase is characterised by microenvironment remodelling and genetic and epigenetic modifications in different cells involved in this disease, causing a gradual variation in cell phenotype and function [312-314]. The accumulation of the extracellular and intracellular modifications causes high level of chemoresistance as demonstrated by the failure of the current treatments, and in most of the cases leads to intrahepatic and extrahepatic metastases [315].

As previously mentioned, in the majority of the cases HCC is developed following the progression of fibrosis towards cirrhosis. This is due to the chronic parenchymal injury and consequent activation of the inflammatory response that triggers a cascade of events that facilitate the development of HCC [316] (Figure 55). Chronic inflammation is promoted by macrophages and myeloid cells that infiltrate the liver and massively release Growth Factors (GF), including Platelet-Derived Growth Factor (PDGF), Transforming Growth Factor $\beta$ (TGF- $\beta$ ) and Tumour Necrosis Factor (TNF), Chemokines and Cytokines (CK), resulting in the continuous propagation of the tissue repair response. Furthermore, the activation of the inflammatory signalling pathways leads to an increased production of Reactive Oxygen Species (ROS) and Reactive Nitrogen Species (NOS) that cause DNA damage and mutations, promoting the neoplastic transformation of hepatocytes [317, 318]. Moreover, the chronic inflammation response causes activation of hepatic stellate cells with subsequent increase of ECM deposition, particularly collagen type I, II and III [319, 320]. The increased ECM production and tissue remodelling, in combination with inflammatory factors cause a continuous parenchymal damage leading to the death and necrosis of hepatocytes, and at the same time, inducing neoplastic hepatocytes to over-proliferate [321]. Furthermore, several studies demonstrated that also Kupffer cells play a key role in the inflammatory response and HCC development. Indeed, the death of hepatocytes activates the neighbouring Kupffer cells, resulting in an increased production of several Cytokines and Growth Factors, including Hepatocyte Growth Factor (HGF), Interleukin-6 (IL-6), and Tumour Necrosis Factor (TNF), which further promote the inflammatory response and enhance the proliferation rate of surviving 
hepatocytes [322]. The hepatocytes' death and regeneration increase the risk of DNA damage and mutations that can eventually lead to HCC [323]. Beside the hepatic stellate cells, that are the first stromal cells to be activated during the progression of fibrosis towards cirrhosis, several other fibroblast derived cell populations contribute to the heterogeneity of HCC. These cells include Cancer Associated Fibroblasts (CAFs) [324], Tumour Associated Endothelial Cells (TAECs) [325] and Myofibroblasts $\alpha$-Smooth Muscle Actin ( $\alpha$-SMA) positive cells [326] which all play a key role in the deposition of ECM and in the development of HCC [327]. Indeed, the qualitative and quantitative modifications of the ECM induced by the activation of these stromal cells increase the stiffness of the organ, producing mechanical stimuli that lead to changes in gene expression and in phenotype of the cells [327].

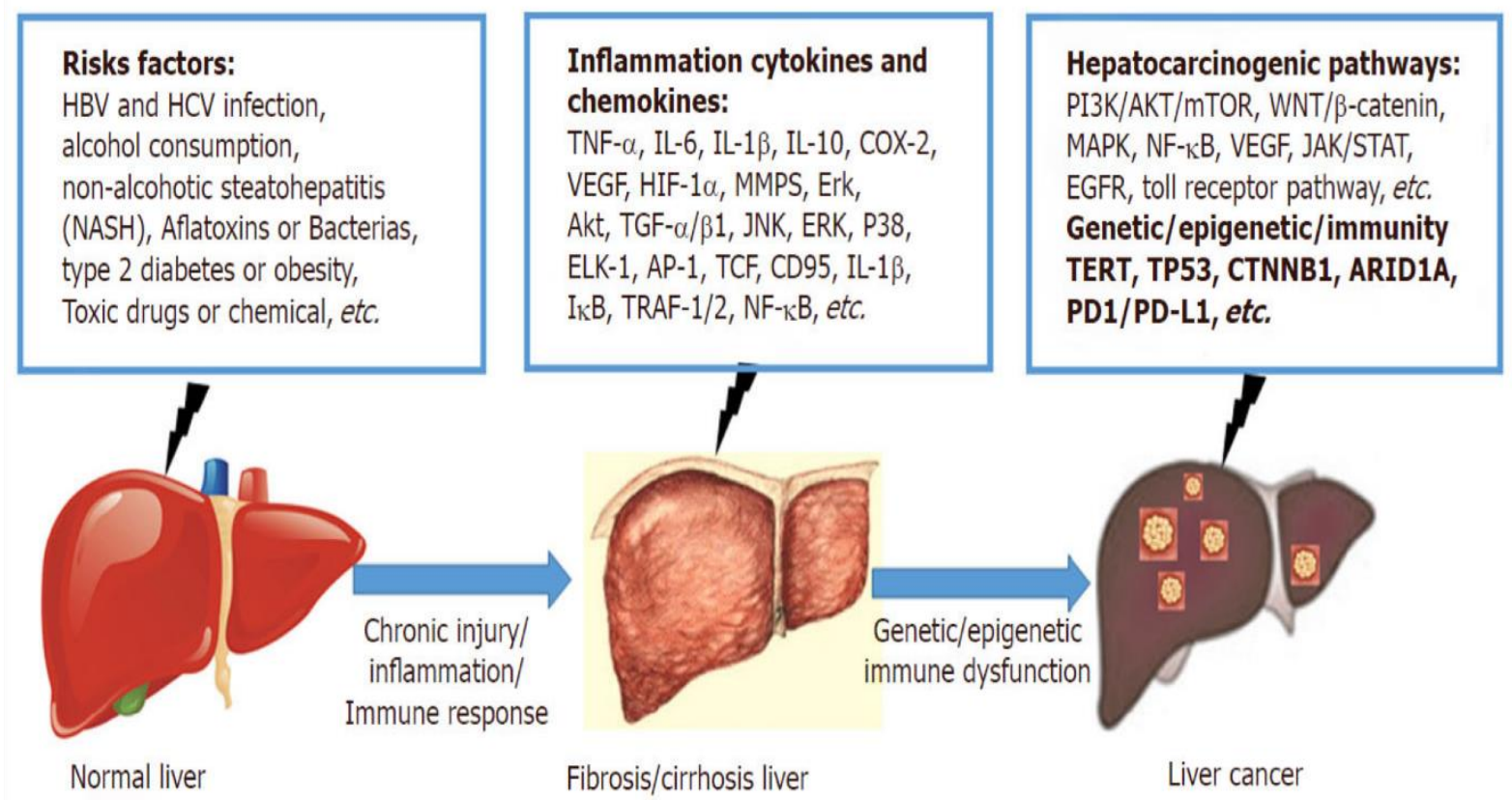

Figure 56. Diagram of $\mathrm{HCC}$ development following the progression of fibrosis towards cirrhosis fibrosis. Image from Hong-Jin Chen et al. [328].

\subsubsection{Current Treatments for HCC}


Several factors involved in the HCC development and disease progression have hampered the development of efficient treatments in order to increase the survival rate correlated to this liver disease.

Available HCC treatments can be classified in two main groups, surgical and systemic treatments. Surgical procedures are most efficient treatments of patients with hepatocellular carcinoma (HCC) and they are usually performed when the tumour is diagnosed in an early phase [329], while patients diagnosed with an advanced stage of HCC are eligible for systemic treatment [330].

\subsubsection{Surgical Treatment}

The three main surgical procedures are tumour ablation, partial liver resection or liver transplantation.

Liver transplantation is the most efficient treatment for patients with a small HCC [331]. Unfortunately the high cost of the procedure, compatible donor organ availability and the restrictive Milan inclusion criteria, such as the presence of a single tumour nodule less than $5 \mathrm{~cm}$ in diameter or a maximum of three nodules having a diameter less than $3 \mathrm{~cm}$ without vascular infiltration [332], limit its eligibility to less than $30 \%$ of patients [333]. In the case of large HCC nodules, liver transplantation is the only effective treatment [334].

Under partial liver resection only the malignant area of the liver is removed, allowing the unresected portion of the organ to regenerate its mass and functionality [334]. This procedure is less effective compared to liver transplantation and is also characterised by a high rate of tumour recurrence [335].

HCC ablation treatments are much less invasive procedures compared to liver transplantation or partial liver resection and are aimed at destroying the tumours in situ. These techniques are performed in patients with small tumours (less than $5 \mathrm{~cm}$ in size), or in patients non-eligible to liver surgery due to compromised liver functions 
[336]. These techniques are also used as a bridge in patients waiting for liver transplantation [307]. HCC ablation treatments include:

[337].

Transarterial Chemoembolization. This treatment is based on the embolization of the hepatic artery in order to limit the oxygen and nutrients supply to the tumour and to induce necrosis [338].

Percutaneous Local Ablation. This treatment is based on the use of radiofrequency that increase the temperature in the local area above $60^{\circ} \mathrm{C}$ causing cellular death. The main limitation of this technique is that the increased temperature damages also the tissues surrounding the tumour [337].

Microwave Ablation. This treatment is based on the use of electromagnetic waves that increase the temperature in located areas above $100^{\circ} \mathrm{C}$ causing cellular death. Similar to Percutaneous Local Ablation, the high temperatures generated using the electromagnetic waves damages the area surrounding the tumour [339].

\subsubsection{Systemic Treatment}

As previously mentioned, patients diagnosed with an advanced stage of HCC are eligible only for systemic treatment with chemotherapeutical drugs.

Sorafenib (Nexavar ${ }^{\circledR}$ ) is the first-line therapy in patients with advanced hepatocellular carcinoma [340]. The anti-cancer effect of Sorafenib is based on inhibiting several kinases involved in tumour proliferation, such as RAF, MEK and ERK, and in the angiogenesis process, such as Raf, VEGFRs and PDGFR- $\beta$, inducing tumour cell apoptosis [341, 342].

In different randomized, placebo-controlled clinical trials, patients were treated with $400 \mathrm{mg}$ of Sorafenib twice a day and results demonstrated that this chemotherapeutical drug prolonged the survival rate in patients with advanced HCC. Indeed, the average survival for sorafenib-treated patients was approximately 11 months while it was less than 8 months in the case of placebo-treated patients [343]. 
Unfortunately, several studies have demonstrated that Sorafenib resistance is common in HCC cancer cells [344]. This process is characterised by a progressive reduced efficacy of the treatment eventually causing tumour progression and metastasis [345].

Regorafenib (Stivarga $\AA$ ) is a systemic chemotherapeutical molecule that acts as an inhibitor of multiple kinases, and it is the second line drug approved for the treatment of HCC in patients having tumour progression during or after sorafenib therapy [346]. Its approval was based on the results achieved during a phase III trial in patients with HCC who had progressed during Sorafenib therapy, where Regorafenib showed to improve the overall survival of patients with advanced $\mathrm{HCC}$, with approximately $50 \%$ of these patients achieving disease control, while the remaining $50 \%$ patients experienced disease progression [347].

Other kinase inhibitors, such as Brivanib, Linifanib, Erlotinib, Dovitinib, or combinations of these chemo drugs, are currently tested in several clinical trials (phase I, II and III). Unfortunately, results obtained so far showed patients treated with these new kinase inhibitors did not experience a prolonged survival when compared to Sorafenib treated patients [348-351].

\subsubsection{Epithelial to Mesenchymal Transition}

Epithelial to Mesenchymal Transition (EMT) is a reversible process in which epithelial cells switch to a mesenchymal phenotype associated with high motility and invasive properties [352]. The EMT is a multi-step process in which cells gradually change from an epithelial phenotype, to an EMT-induced phase, characterised by the expression of both epithelial and mesenchymal markers, to a further mesenchymal phenotype. As previously mentioned, the EMT is a reversible process that can be shifted back through a process called Mesenchymal to Epithelial Transition (MET). Several studies showed that both EMT and MET play a key role in tumour growth, colonization and in metastases development (Figure 56) [353, 354]. 
In the case of HCC, the adverse tumorigenic microenvironment, based on inflammation, hypoxia and increased production of radical species including reactive ROS and NOS species, drives the hepatocytes to initiate the EMT process characterised by progressive loss of their epithelial markers, such as E-Cadherin and Intermediate Filaments (Keratin 8 and 18). The latter are essential to maintain cell to cell adhesion, and at the same time, these hepatocytes start progressively to express mesenchymal markers, such as Vimentin and $\alpha$-Smooth Muscle Actin ( $\alpha$-SMA), which confer them high motility and invasive properties [355, 356]. Such motility allows the hepatocytes to escape from the adverse microenvironment and migrate towards chemokine and cytokine rich environments where the neoplastic cells reverse their mesenchymal phenotype through MET and initiate the colonization process that leads to the formation of tumour metastases [357].

Transforming Growth Factor $\beta$ (TGF- $\beta$ ) is one of the key factors driving the hepatocytes EMT process [358, 359]. The most studied TGF- $\beta$ isoforms are TGF- $\beta 1$, 2 and 3 , and it has been shown that these ligands are able to trigger two different signalling cascades following the binding with TGF- $\beta$ receptors [360]. The first one, known as canonical TGF- $\beta$ signaling pathway, is based on the phosphorylation and activation of SMAD [361]. The second cascade, known as the non-canonical TGF- $\beta$ signalling pathway, is based on the phosphorylation of MAPK, ERK and MEKK [362, 363].

Both TGF $\beta$ signalling pathways regulate the expression of several transcriptional factors involved in the EMT process [364]. These factors, such as Snail [365], Slug [366], and ZEB1 [367] are able to coordinate a transcriptional network which leads in a downregulation of epithelial markers, such as E-Cadherin, and upregulation mesenchymal markers, such as Vimentin, Fibronectin and $\alpha$-Smooth Muscle Actin, with consequent loss of cell polarity, dissolution of cell to cell adhesion junctions and increased cell migration [368].

Another key transcriptional factor involved in the EMT process is Twist [369], which expression is mainly regulated through Wnt and EGFR signalling pathways [370]. Activation of Twist causes a downregulation of E-Cadherin expression, thus leading to a more mesenchymal phenotype. 
Beside the previously mentioned EMT markers, including E-Cadherin, Vimentin, Fibronectin and $\alpha$-Smooth Muscle Actin, another important marker of this transition is $\beta$-Catenin, which is found up-regulated in several tumours including $\operatorname{HCC}(71)$. $\beta$ Catenin expression is regulated through both Wnt and TGF $\beta$ signalling pathways [371, 372]. In normal Hepatocytes, characterised by an epithelial phenotype, $\beta$-Catenin is phosphorylated to create a complex with E-Cadherin providing anchoring junctions for the actin filament of the cytoskeleton that are essential to maintain cell adhesive properties and to transduce intra- and inter-cellular signalling pathways [116, 373]. During EMT, E-Cadherin is downregulated and the overexpressed $\beta$-Catenin is translocated to the nucleus where it induces the expression of several genes leading to an abnormal cellular proliferation and metastatic behaviour [374].

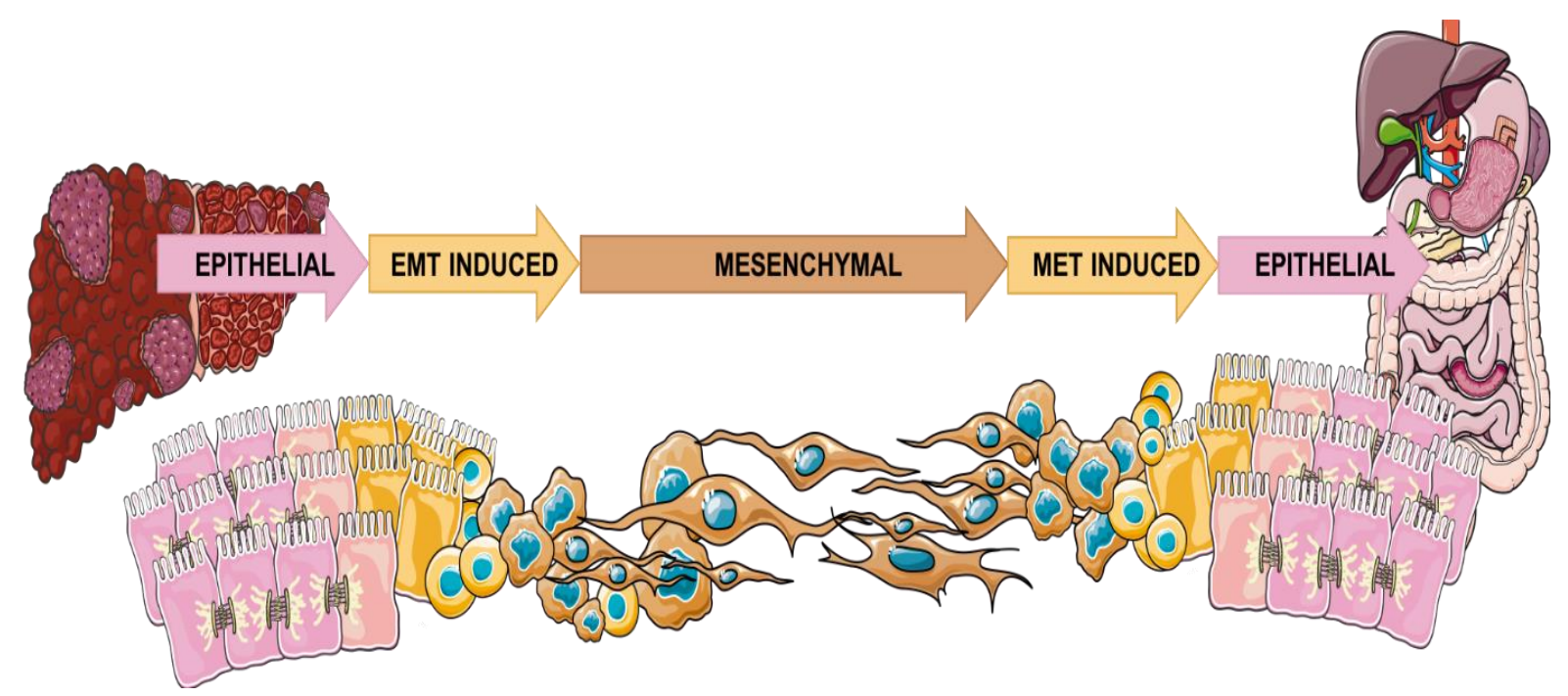

Figure 57. EMT/MET processes. Adverse microenvironment drives epithelial cells switch to a mesenchymal phenotype (EMT), promoting cell migration and colonisation of neighbouring tissues or organ where the epithelial phenotype is reversed [353].

\subsubsection{Hepatocellular Carcinoma SNU and HepG2 Cell Lines}

Seoul National University (SNU) is a well-known institution due to their massive effort in developing new cell lines. Indeed, since 1982 more than 100 cell lines were derived from primary cancer of Korean patients, including 12 cell lines derived HCC [375]. 
These 12 characterised HCC SNU cell lines are marked with several differences in the genetic profile which are tightly related to the developmental stage of the HCC.

One of the most commonly used SNU HCC cell lines is SNU-449, mainly due to its genetic profile characteristics and because it has been listed in the American Type Culture Collection (ATCC, Rockville, MD, USA), thus facilitating their use for research studies. The SNU-449 cell line was derived in 1990 through Hepatitis B Viral infection and consequent integration in the genome that caused mutations in p53 gene, specifically in the codon 138 [376, 377]. Mutations in p53 gene are detected in more than $30 \%$ of $\mathrm{HCC}$ patients [378], with an even higher percentage in Asian and African countries where Hepatitis B and C viral infections are more common [379]. This set of characteristics makes SNU-449 cells suitable for the in vitro study of HCC development and HCC chemoresistance.

Similar to SNU-449, HepG2 cell line has been listed in the ATCC repository (American Type Culture Collection, Rockville, MD, USA) making them accessible for research purposes. The classification of the HepG2 tumour of origin has risen questions between investigators. Indeed, when screening the scientific literature, it is possible to find HepG2 references for both hepatocellular carcinoma and hepatoblastoma studies. Hepatoblastoma is an embryonal malignancy of hepatocellular origin usually developed in the first years of life [380]. Paediatric hepatocellular carcinoma is a rare tumour characterised by a much worse aggressive behaviour and less responsive to chemotherapy compared to a hepatoblastoma. Moreover, the distinction between these tumours in older children can be difficult [381].

\subsubsection{In Vivo and In Vitro HCC Models}

The absence of efficient models able to recapitulate the human HCC pathophysiology in terms of cellular components and its specific microenvironment, limits the development of efficient drugs for the treatment of this hepatic tumour. Indeed, models that lack important parameters, such as mechanical properties, oxygen and nutrients diffusion, human tissue specific cell-cell interactions and cell-ECM interactions [382, 
383] can lead to false results regarding the toxicity and efficacy of new therapeutic drugs [98].

Available models used to study HCC and to develop new drugs can be divided in two classes: in vivo and in vitro models.

\subsubsection{In vivo models}

The laboratory mouse (Mus musculus) is considered the best in vivo model to study the cancer pathophysiology due to its breeding capacity, size, and availability of multiple gene manipulations that enhance its similarities to human biology [384].

Mus musculus in vivo models can be divided in four types according to the techniques performed to induce the tumour [385-387]:

Chemically Induced Models: The HCC in the mice is induced through the employment of several toxic chemical compounds. Carcinogenic compounds can be classified in two groups depending on their mechanism of tumour generation: (i) genotoxic compounds which directly induce DNA damage and (ii) promoting compounds which do not have a direct genotoxic capability but enhance tumour formation triggering chronic inflammation and tissue damage [385]. Some of the most common used chemicals are Diethylnitrosamine (DEN) [388], 2-Acetylaminofluorene (2-AAF) [389], Thioacetamide (TAA) [390] and Aflatoxins [391]. The Aflatoxins are considered the most hepatotoxic agents, and both International Agency of Research on Cancer (IARC) and U.S. National Toxicology Program (NTP) listed these agents as Class I, meaning that they are carcinogenic to humans.

Syngeneic and Xenograft Models: These in vivo models are obtained by transplanting HCC cell lines into immune or non-immunocompetent mice. HCC cell lines can be transplanted under the skin (ectopic) or directly into the liver (orthotopic transplantation) [392]. This leads to a syngeneic model (also called allograft models) when murine cell lines are transplanted into the mice to develop the tumour. The most common murine cell lines used to develop syngeneic model are Hepa I, MH129 and $\mathrm{MH} 134$ [386]. On the contrary, xenograft models are originated when human HCC cell 
lines are transplanted in immunodeficient (nude) mice. Human cell lines used to develop HCC xenograft models include HepG2 and Hep3B and PLC5 [386].

Genetically Engineered Models (GEMs): These transgenic mice are originated through genetic modifications, mainly via knock out and knock in of different genes, that allow to better understand the molecular and cellular mechanisms underlying the cancer process, to identify and to validate novel cancer genes and tumour biomarkers [393].

Although these animal models have been extensively used to study the mechanisms underlying the $\mathrm{HCC}$ and as predictive tools to evaluate the efficacy and safety of new therapeutic treatments before been tested in humans, they are characterised by several limitations such as $I$ the fact that they are not able to fully mimic the human microenvironment of disease and II) interspecies differences such as anatomy, size and metabolic activity [125]. These differences between the animal models and humans reflect the difficulty in developing efficient HCC treatments. Indeed, $90 \%$ of the drugs that successfully complete pre-clinical trials in animal models, do not provide a beneficial outcome and/or are characterised by unacceptable toxicity when tested in humans [394-396]. Furthermore, considering the large number of drugs that fail during clinical trials, the use of animal models as predictive tools for clinical outcome results a non-cost-effective process.

\subsubsection{In vitro Models}

Although in vivo models have been widely used in the field of HCC research, the lack in human specific cells and ECM, does not allow to fully recapitulate the specific human HCC pathophysiology [382, 383, 397]. In vitro models play a crucial role in translational medicine and can bridge the gap between basic research and clinical benefits.

During the past years, different methods have been employed for the production of in vitro HCC models. These include: 
Organ on a chip: this model is based on the use of interconnected 3D microfluidic cell culture chips that allow to control the flow and the exchange rate of different substances including oxygen, nutrients, waste products and chemical agents [398] simulating the activity and functionality of human organs [399]. Regarding the topic related to the study of this thesis, HCC cell lines and stromal cells were co-cultured in these models to better mimic the cell heterogeneity of human HCC $[400,401]$. The usage of porous polymeric membranes to culture the cells is the main limitation of these models. Indeed, the absence of tissue specific ECM limits the ability of these in vitro models to mimic the particular human HCC microenvironment.

Precision Cut Liver Slices: With regard to HCC, this model has been mainly used for drug screening by preparing micro-slices of the tumorous tissue [402]. Precision Cut Liver Slices overcome the limitations of other models as they allow the study of solid tumour with preservation of the tissue architecture, cell-cell and cell-matrix interaction. The major limitation of this in vitro model is the restricted preservation time, up to 48 hours [147].

Cell Sheet Stacking: in the case of HCC research, the HepG2 cell line in co-culture with stromal cells have been used to produce these cell sheets stacked on top of each other to create the 3D structure [403]. The main limitation of these models based on the use of synthetic polymers, such as poly ( $\mathrm{N}$ - isopropylacrylamide) (PNIPAAm), is the lack of tissue specific ECM to better mimic the human liver microenvironment.

Cell Spheroids: in the context of HCC, Matrigel (Corning) was used in several studies to develop HCC cell line spheroids [404, 405]. However, as previously mentioned in animal origin thus does not accurately represent the human tumour microenvironment. Indeed, Matrigel matrix is a reconstituted basement membrane preparation extracted from the Engelbreth-Holm-Swarm mouse sarcoma. This material, once isolated, contains approximately $60 \%$ laminin, $30 \%$ collagen IV, and $8 \%$ entactin.

Scaffold based 3D models: regarding the study of HCC, it is possible to find different studies mainly based on the use of synthetic porous hydrogels [406] or natural polymer hydrogels [407] to study parameters of the HCC cells such as drug resistance and phenotype. However, these 3D models are not able to recapitulate the human liver microenvironment. On the contrary, decellularised 3D scaffolds are based on the 
removal of cellular materials from an organ while preserving the mechanical and bioactive characteristics [408]. One of the major advantages of decellularised models is the preservation of the ECM components and the micro-architecture of the original native organs. The major limitations of the decellularised models are the engraftment capacity which depends on the cell type and the highly time-consuming process to develop such models.

The absence of efficient in vivo and in vitro models has become a major barrier for the identification of new targets and the development of new drugs, causing only a modest improvement in beneficial outcome in patients. 


\subsection{Aim and objectives}

\subsubsection{Aim}

To develop, optimise and validate a novel 3D in vitro model for the study of HCC and for testing anti-cancer treatments.

\subsubsection{Objectives}

To achieve the aim mentioned above the following objectives will be considered:

1. Assessing the in vitro biocompatibility of the thermoresponsive ECM-based hydrogels.

2. Investigate the maintenance of an increasing proliferation and metabolic activity, induced by spheroid formation, during at least 13 days of 3D culture.

3. Validate the anti-cancer predictive efficiency of the developed model using Sorafenib, the first-line treatment for patients with advanced HCC.

4. Validate the efficiency of the developed model through the TGF- $\beta 1$ treatment to induce EMT within the tested HCC cell line. 


\subsection{Methods and Materials}

\subsubsection{Preparation of Human Liver ECM solution}

Human livers were decellularized following a novel protocol developed and published by Mazza et al. in 2015. Next, the human decellularized liver was lyophilized (VIRTIS Benchtop) overnight to obtain a dried tissue that was then milled (rotary knife milling machine, Wiley Mini-Mill) to create human liver ECM powder. Human liver ECM powder was solubilised in $0.5 \mathrm{M}$ acetic acid (Thermo Fisher Scientific) $(250 \mathrm{ml}$ for $1 \mathrm{~g}$ of ECM powder), without the implementation of pepsin, at $4{ }^{\circ} \mathrm{C}$ for 72 hours under magnetic stirring. Human liver ECM solution was then ultra-centrifuged (Beckman Coulter, LE- $80 \mathrm{~K}$ Ultracentrifuge) at $10000 \mathrm{~g}$ at $4{ }^{\circ} \mathrm{C}$ for 30 minutes. The pellet of human liver ECM was resuspended in acetic acid $0.25 \mathrm{M}$ (166.6 $\mathrm{ml}$ for $1 \mathrm{~g}$ of ECM powder) at $4{ }^{\circ} \mathrm{C}$ for 24 hours followed by sonication (Bandelin Sonoplus) ( 5 cycles of 20 seconds at $50 \mathrm{~Hz}$ ). The $\mathrm{pH}$ of the resultant ECM solution was then neutralised with $10 \mathrm{~N}$ sodium hydroxide (30 $\mu \mathrm{l}$ per each $\mathrm{ml}$ of ECM solution) and the osmotic pressure was adjusted with 10X PBS (1:10 volume of ECM solution). This human liver ECM solution, characterised by an ECM concentration of $5,3 \mathrm{mg} / \mathrm{ml}$, was sterilised overnight by gamma-irradiation (1782 Gy, using CIS bio international IBL $437 \mathrm{C}$ irradiator) at the Institute of Child Health (ICH) facilities at UCL.

\subsubsection{Maintenance of hepatic cells in culture}

HepG2 cells and SNU-449 cells were used for this study. Both HepG2 and SNU-449 cells lines were cultured in MEM Medium (Gibco Thermo Fisher Scientific) supplemented with 10\% Foetal Bovine Serum (FBS; Gibco Thermo Fisher Scientific), 2mM L-glutamine (200mM Gibco Thermo Fisher Scientific), 1\% Sodium Pyruvate (100X Gibco Thermo Fisher Scientific), 1\% Non-Essential Amino Acids (100X Gibco Thermo Fisher Scientific) and 1\% 1X Antibiotic-Antimycotic (100X Gibco Thermo 
Fisher Scientific). These hepatic cell lines were cultured under standard conditions in a humidified incubator at $37{ }^{\circ} \mathrm{C}$ with $5 \% \mathrm{CO}_{2}$. Once cells reached $\sim 75 \%$ confluency, cells were trypsinised using $0.25 \%$ Trypsin-EDTA (Thermo Fisher Scientific) and passaged at a split ratio of $1: 3$.

\subsubsection{Hep-Gel Preparation}

Hep-Gel was prepared by combining the human liver ECM solution with Worm-gel solution in ratio 1:1 (v/v). Worm-gel is a synthetic thermoresponsive copolymer (2hydroxypropylmethacrylate and Poly-glycerolmonomethacrylate) kindly provided by Professor Battaglia, UCL Chemistry department. At low temperature this synthetic copolymer is characterised by a liquid state but turns into a gel when the temperature increases above $21^{\circ} \mathrm{C}$ [409]. For this reason, to prepare the Hep-Gel, both Worm-gel and human liver ECM solutions were kept in ice inside the cell culture cabinet. This was followed by a mix of the desired volume of two solutions by pipetting in ice.

From this point onwards, the following definitions will apply:

Hep-Gel: is the combination of Worm-gel with human liver ECM solution

Control-Gel: is composed of Worm-gel only and used as internal control

\subsubsection{D determining the optimal cell density in Hep-Gel}

Different amount of HepG2 cells were embedded and cultured in Hep-Gel for 7 days in order to define the best cell density in the biomaterial and investigated as followed:

- $0.33 \times 10^{3}$ cells $/ \mu$ l of biomaterial corresponding to $0.5 \times 10^{5}$ HepG2 in $150 \mu \mathrm{l}$ of biomaterial.

- $1.66 \times 10^{3} \mathrm{cells} / \mu \mathrm{l}$ of biomaterial, corresponding to $2.5 \times 10^{5} \mathrm{HepG} 2$ in $150 \mu \mathrm{l}$ of biomaterial. 
- $3.3 \times 10^{3} \mathrm{cells} / \mu \mathrm{l}$ of biomaterial, corresponding to $5 \times 10^{5} \mathrm{HepG} 2$ in $150 \mu \mathrm{l}$ of biomaterial.

- $5 \times 10^{3}$ cells $/ \mu \mathrm{l}$ of biomaterial, corresponding to $7.5 \times 10^{5} \mathrm{HepG} 2$ in $150 \mu \mathrm{l}$ of biomaterial.

Live/Dead staining confirmed that $0.33 \times 10^{3}$ cells/ $\mu$ l of Hep-Gel was the best cell density and for this reason it was selected to perform all the further experiment described in this thesis. Furthermore, considering the high cell viability and proliferation rate, the size of each dispended Hep-Gel was reduced from $150 \mu \mathrm{l}$ to 50 $\mu \mathrm{l}$, corresponding to a total amount of $0.165 \times 10^{5} \mathrm{HepG} 2$ in each Hep-Gel. This allowed to reduce the number of cells and biomaterial needed to perform the experiments.

\subsubsection{D cell culture in Hep-Gel or Control-Gel}

HepG2 or SNU-449 cells were then trypsinised and counted to obtain a total amount of cell corresponding to the desired cell density of $0.33 \times 10^{3}$ cells per microliter $(\mu \mathrm{l})$ of biomaterial. A maximum of $1.5 \mathrm{ml}$ of Hep-Gel or Control-Gel were used in each independent experiment with a total cell density of $0.495 \times 10^{6} / 1.5 \mathrm{ml}$ solution. After centrifugation, the required cell amount was resuspended in a volume of 150 microliters $(\mu \mathrm{l})$ of complete medium $(100 \mu \mathrm{l}$ per $\mathrm{ml}$ of biomaterial). The cell resuspension was then mixed with Hep-Gel or Control-Gel in a liquid state by pipetting on ice. Fifty microliters $(\mu \mathrm{l})$ of $\mathrm{Hep}-\mathrm{Gel}$ or Control-gel solutions were then dispensed in each well of a pre-warmed 48-well plate followed by an incubation of 5 minutes inside a humidified incubator at $37^{\circ} \mathrm{C}$ with $5 \% \mathrm{CO}_{2}$ to allow the complete gelation of the biomaterials. Finally, $1 \mathrm{ml}$ of pre-warmed cell culture medium was added to each HepGel or Control-Gel.

Cell culture medium used for both HepG2 and SNU-449 cell lines was MEM Medium supplemented with 10\% Foetal Bovine Serum, 2mM L-glutamine, 1\% Sodium Pyruvate, 1\% Non-Essential Amino Acids and 1\% 1X Antibiotic-Antimycotic. Samples were kept in culture inside a humidified incubator at $37{ }^{\circ} \mathrm{C}$ with $5 \% \mathrm{CO}_{2}$ up to 13 days replacing the complete medium every other day. 
During cell culture, cell supernatants were collected and stored at $-20^{\circ} \mathrm{C}$ for further cell functionality evaluation.

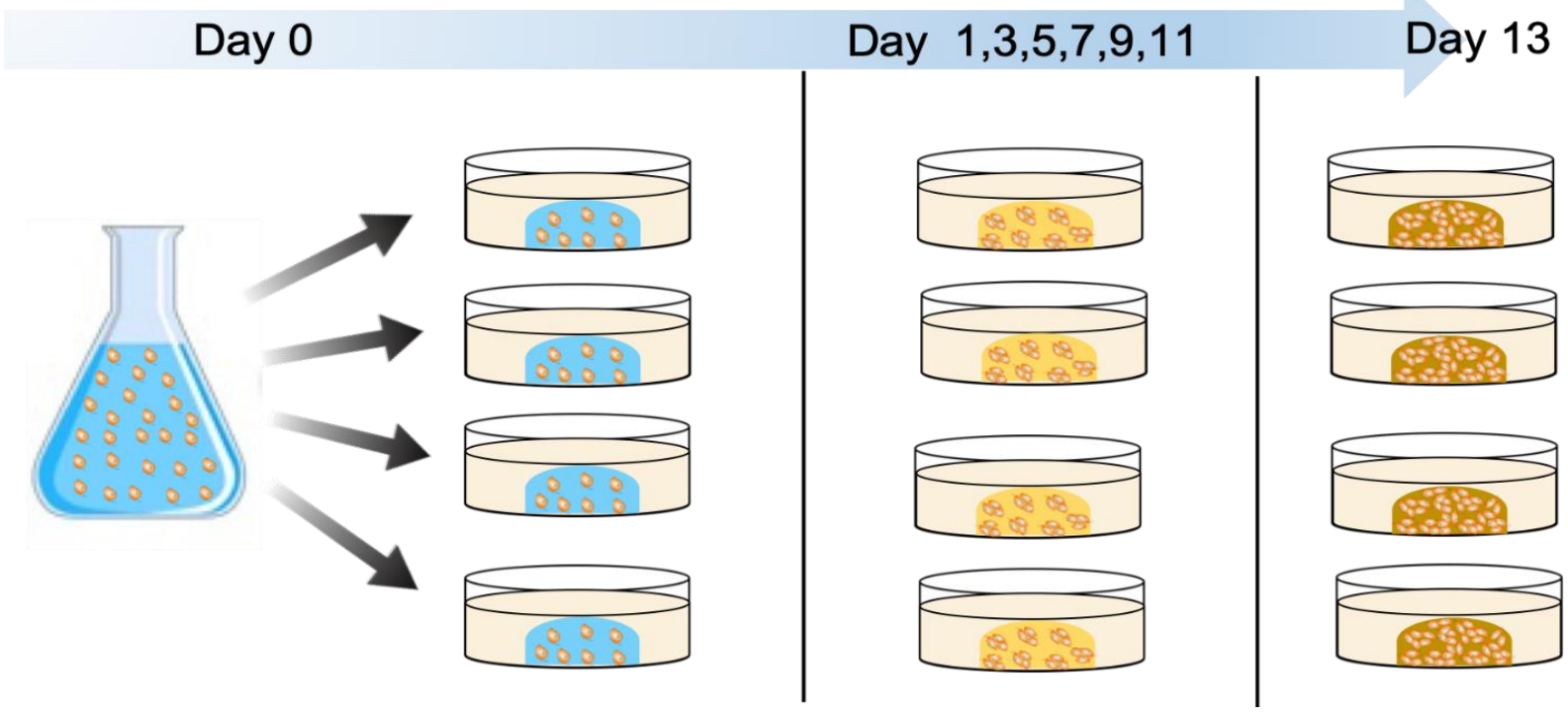

Figure 58. Schematic representation of the timeline for 3D cell culture in Hep-Gel or ControlGel. Hepatic cells are first mixed with the Hep-Gel or Control-Gel in a liquid state and then 50 $\mu \mathrm{l}$ of biomaterial is dispended in each well of a 48 well plate. One $\mathrm{ml}$ of cell culture medium is added in each well which is replaced every other day during the cell culture.

\subsubsection{Live/Dead cell double staining}

Cell viability was measured employing the Live/Dead fluorescence double staining assay. This technique is based on the use of Propidium lodide (PI) (Sigma-Aldrich), an intercalating red fluorescent agent that binds only to the DNA of apoptotic cells since it cannot cross the membrane of living cells, and Fluorescein Diacetate (FDA) (Sigma-Aldrich), a non-fluorescent agent that in the nucleus of alive cells is converted to fluorescein, a green fluorescent compound.

Live/Dead cell double staining was performed by adding $500 \mu \mathrm{l}$ of pre-warmed $1 \mathrm{X}$ HBSS (Gibco) containing 4\% Propidium lodide (PI) and 2\% Fluorescein Diacetate (FDA) to each Hep-Gel or Control-Gel bioengineered with HepG2 or SNU-449. The samples were incubated in the dark for 1 minute at room temperature followed by 
several washes with 1 X HBSS to remove any non-adherent dyes. Samples were then visualised under fluorescent microscope (Nikon model U-III equipped with Nikon mercury lamp) and analysis of the captured images was performed with Fiji v1.49d (ImageJ Jenkins server).

\subsubsection{Presto blue viability test}

Compared to the previous Chapters, PrestoBlue ${ }^{\circledR}$ (Thermo Fisher Scientific) was used to evaluate cell viability instead of AlamarBlue. Both reagents are based on the conversion of resazurin, a blue and weakly fluorescent compound, to resorufin, a pink and fluorescent compound. In the in the case of PrestoBlue this conversion is faster and therefore it allows to reduce the incubation time.

Presto Bluecell viability reagent $10 \mathrm{X}$ was first diluted $1: 10$ with cell culture medium and then $1 \mathrm{ml}$ of resulting solution was added to each Hep-Gel or Control-Gel bioengineered with HepG2 or SNU-449. Samples were then incubated for 2 hours in a humidified incubator at $37^{\circ} \mathrm{C}$ with $5 \% \mathrm{CO}_{2}$. Quadruplicates of each sample $(200 \mu \mathrm{l}$ per well) were analysed in a 96 opaque black well plate (Thermo Fisher Scientific). Microplate Reader (Infinite M200 PRO Multimode Microplate reader, Tecan, Switzerland) was used to measure the absorbance at $570 \mathrm{~nm}$.

When performing experiments with hepatic cell cultures in the conventional 2D plastic wells, Presto Blue $\AA^{\circledR}$ cell viability reagent $10 \mathrm{X}$ was first diluted 1:10 with cell culture medium and then $1 \mathrm{ml}$ of resulting solution was added to each well. Samples were incubated for 30 minutes in a humidified incubator at $37^{\circ} \mathrm{C}$ with $5 \% \quad \mathrm{CO}_{2}$. Quadruplicates of each sample (200 $\mu \mathrm{l}$ per well) were analysed in a 96 opaque black well plate. Microplate Reader (Infinite M200 PRO Multimode Microplate reader, Tecan, Switzerland) was used to measure the absorbance at $570 \mathrm{~nm}$.

\subsubsection{Histology and Immunofluorescence}


Bioengineered Hep-Gel and Control-Gel with HepG2, previously fixed in $10 \%$ formalin (Leica Biosystems), were first washed in distilled water and then dehydrated in a series ethanol (Acquascience) and xylene baths, followed by embedding in paraffin (Leica Biosystems). The samples were sliced into $5 \mu \mathrm{m}$ sections using a microtome (Leica Biosystems Leica RM2035). All sections were then immersed in xylene baths (Acquascience) for at least 5 minutes, and ethanol baths (Acquascience) for at least 2 minutes and then rinsed in tap water.

\subsubsection{Histology}

Sections were stained as follows:

Haematoxylin and Eosin: Sections were first stained with Harris Haematoxylin (Leica Biosystems) for 10 minutes followed by washing in tap water for 5 minutes. Next, the sections were stained with Eosin (Leica Biosystems) for 3 minutes, followed by washing in tap water for 5 minutes. The sections were then quickly dehydrated in ethanol and then immersed in xylene bath (Acquascience) until mounted.

Picro-Sirius Red (SR): Sections were stained with Picro-Sirius Red (R.A.Lamb; Cl35780 ) for approximately 20 minutes. The sections were then quickly dehydrated in ethanol and then immersed in xylene bath (Acquascience) until mounted.

All sections were mounted with DPX (Leica Biosystems) and finally cover slipped. Images were captured with an Axiocam IcC5 using Zeiss Axiovision (verison 4.8.2)

\subsubsection{Immunofluorescence}

Bioengineered Hep-Gel and Control-Gel with HepG2 were first washed twice (5 minutes each time) with 1X PBS (Sigma). Samples were then fixed for 20 minutes in $500 \mu \mathrm{L}$ of $10 \%$ formalin (Sigma) in $1 \mathrm{X}$ PBS at room temperature using an orbital shaker (Ika Labortechnik KS501 digital). This was followed by a washing step in 1X PBS (2 times, 10 minutes). A PBS $1 \mathrm{X}$ solution containing $500 \mu \mathrm{L}$ of $0.5 \%$ Triton-X100 (Sigma) and $3 \%$ BSA (Sigma) was added to each Hep-Gel or Control-Gel for 1 hour 
(permeabilization step performed on the orbital shaker) followed by a washing step with 1 X PBS (3 times, 20 minutes on the shaker).

When performing the Hoechst and Phalloidin staining, bioengineered Hep-Gel with HepG2 were incubated for 1 hour with $500 \mu \mathrm{L}$ of Phalloidin TRICT (Sigma) diluted 1:200 in a solution containing 3\% BSA in PBS 1X solution. This step was performed at room temperature, in the dark and on the orbital shaker. This was followed by washing steps with 1 X PBS (3 times, 20 minutes) on the orbital shaker. Samples were incubated for 20 minutes with $500 \mu \mathrm{L}$ of Hoechst 33258 (Sigma) diluted 1:5000 in PBS $1 X$. This step was performed at room temperature and on the orbital shaker. A washing step with 1X PBS (3 times, 20 minutes on the shaker) was done before the acquisition of the images using a confocal microscope (Leica SP5 inverted confocal microscope, Leica Application Suite X (LAS X) software).

When performing a double staining of Hoechst with EpCAM staining, samples were incubated overnight at $37^{\circ} \mathrm{C}$ and on the orbital shaker with $500 \mu \mathrm{L}$ of EpCAM antibody (Abcam ab71916) diluted 1:200 in in 3\% BSA in PBS 1X solution. This was followed by washing steps with $1 \mathrm{X}$ PBS (3 times, 20 minutes) on the orbital shaker. Samples were incubated with the secondary antibody Dyelight 448 (Biolegend) diluted 1:250 in $3 \%$ Bovine Serum Albumin (BSA) in PBS 1X. This step was done at room temperature, in the dark and on the orbital shaker. This was followed by washing steps with 1X PBS (3 times, 20 minutes) on the orbital shaker followed by an incubation for 20 minutes with $500 \mu \mathrm{L}$ of Hoechst 33258 (Sigma) diluted 1:5000 in PBS 1X at room temperature and on the orbital shaker. A washing step with 1X PBS (3 times, 20 minutes on the orbital shaker) was performed before the acquisition of the images using a confocal microscope (Yokogawa SQ1).

\subsubsection{Human Albumin ELISA}

Secreted albumin by HepG2 cell cultures was assessed in culture supernatants by performing the Human Albumin ELISA assay (Human Albumin ELISA kit ABCAM). Cell supernatants, collected at the end of the experiment (day 13), were diluted 1:60 
for HepG2 samples with Diluent Solution provided in the kit. Fifty $\mu \mathrm{l}$ of diluted cell supernatants and Albumin Standard Solutions were added and incubated in a 96 well plate for 1 hour followed by 5 washes with Washing Buffer. Fifty $\mu$ l of $1 \mathrm{X}$ Biotinylated Albumin Antibody was then added in each well and washed after 30 minutes. Next, 1X SP Conjugate antibody was added and washed after 30 minutes. Next, $50 \mu \mathrm{l}$ of Chromogen Substrate Solution was added in each well and incubated for 25 minutes. Finally, $50 \mu \mathrm{l}$ of Stop Solution was used to block the reaction until the colour of the samples turned from blue to yellow. Absorbance signal related to albumin content was measured at $450 \mathrm{~nm}$ using the plate reader (FLUOstar Omega BMG Labtech).

\subsubsection{Sorafenib Treatment}

HepG2 or SNU-449 cells lines were cultured in Hep-Gels for 7 days before being exposed to the Sorafenib chemo drug. Treatment of the resultant bioengineered HepGels with HepG2 or SNU-449 cell lines was performed applying Sorafenib (Nexavar) at different concentrations i.e. $2.5 \mu \mathrm{M}$ and $10 \mu \mathrm{M}$, corresponding to the clinical dose given to HCC patients (10 $\mu \mathrm{M}$ equivalent to $400 \mathrm{mg} / \mathrm{twice}$ a day) (123). Forty $\mu \mathrm{M}$ Sorafenib was used to investigate the possibility of drug resistance in these cell lines when cultured in the $3 D$ models. The sample size of this experiment was $n=5$ for the untreated control samples as well as each Sorafenib concentration and the incubation time with the drug was 48 hours for all the concentrations.

Following the treatment, cell viability was immediately analysed through Presto Blue $(n=4)$ and Live/Dead staining $(n=1)$.

When analysing Sorafenib in 2D plastic cultures, $0.3 \times 10^{6}$ of HepG2 or SNU-449 were plated in each well of a 12 well-plate ( $n=4$ for each condition). After $24 \mathrm{hrs}$ in culture cells were treated with Sorafenib at $2.5 \mu \mathrm{M}$ for 48 hours. Following the treatment, cell viability was immediately analysed through Presto Blue $(n=4)$ and bright-field microscopy images (Nikon microscope model U-III). 


\subsubsection{TGF- $\beta 1$ Treatment}

To investigate the EMT in SNU-449 cells, cells were cultured in Hep-Gel, and the EMTinducing growth factor TGF- $\beta 1$ was added ( $5 \mathrm{ng} / \mathrm{ml}$, R\&D Systems). SNU-449 cells were cultured in Hep-Gel for 7 days before being exposed to TGF- $\beta 1$. Treatment of the samples $(n=5)$ with TGF- $\beta 1$ was performed for 48 hours. This was followed by analysis of cell viability through Live/Dead staining $(n=1)$ and $\mathrm{GPCR}$ to evaluate differences in the gene expression compared to the untreated control samples $(n=4$ for both TGF- $\beta 1$ treated and untreated Hep-Gel).

\subsubsection{RNA extraction, reverse transcription and gene expression analysis}

\subsubsection{RNA extraction}

RNeasy mini Kit (Qiagen) was employed to extract RNA from the SNU-449 cells grown in Hep-Gel. The previously snap frozen bioengineered Hep-gel were first placed for 30 minutes in ice to allow the Hep-Gel to turn into a liquid state. Lysis Buffer (Qiagen) containing $1 \%$ of $\beta$-mercaptoethanol (Sigma-Aldrich) was then added to the samples followed by vigorously pipetting to further disrupt the samples. As indicated in the protocol provided by the manufacturer, consequents steps were performed using spin columns and different ethanol-based buffers. RNA extracted from Hep-Gel was measured at $260 \mathrm{~nm}$ with the spectrophotometer (NanoDrop, ThermoScientific).

\subsubsection{Complementary DNA (cDNA) synthesis}

Complementary cDNA reverse transcription was performed using high Capacity cDNA Reverse Transcription Kit (Applied Biosystems). The extracted RNA was diluted in RNase free water (Qiagen) in order to obtain the same RNA concentration in all the samples. Subsequently, $10 \mu \mathrm{l}$ of the samples' RNA was added in independent PCR microtubes and then mixed with $10 \mu$ of Master Mix, which composition is shown in 
the table 16. The reverse transcription was performed using the 2720 Thermal Cycler (Applied Biosystems) following the program described in table 17.

\begin{tabular}{|l|l|}
\hline RT Master Mix reagents & \multicolumn{1}{|l|}{ Volume $(\mu \mathrm{L})$} \\
\hline 10X RT Buffer & 2 \\
\hline 25X dNTPs Mix (100 mM) & 0.8 \\
\hline 10X RT Random Primers & 2 \\
\hline MultiScribe RT & 1 \\
\hline RNase Inhibitor & 1 \\
\hline Nuclease Free $\mathrm{H}_{\mathbf{2}} \mathbf{0}$ & 3.2 \\
\hline
\end{tabular}

Table 16. RT Master Mix composition.

\begin{tabular}{|l|l|}
\hline Step 1 & 10 minutes at $25^{\circ} \mathrm{C}$ \\
\hline Step 2 & 120 minutes at $37^{\circ} \mathrm{C}$ \\
\hline Step 3 & 5 minutes at $85^{\circ} \mathrm{C}$ \\
\hline Step 4 & Hold at $4^{\circ} \mathrm{C}$ \\
\hline
\end{tabular}

Table 17. Steps of Reverse transcription program using 2720 Thermal Cycler. 


\subsubsection{Quantitative Real-Time PCR (RT- qPCR)}

The obtained cDNA was first diluted in RNase free water to get a final concentration of $2 \mathrm{ng} / \mu \mathrm{l}$. $10 \mathrm{ng}$ of cDNA from each Hep-gel was added into a Fast Optical 96-well TaqMan PCR Plate (MicroAmp, Applied Biosystems) and then mixed with $15 \mu \mathrm{l}$ of qPCR Master Mix, which composition is described in table 18. Last, the PCR Plate was inserted in $\mathrm{ABI} 7500$ Fast Real Time PCR System (Applied Biosystems) to perform 40 cycles of cDNA amplification (table 19).

Comparative CT method was used to analyse the relative expression of the investigated genes (table 20) using Glyceraldeyde-3-phosphate dehydrogenase (GAPDH) as a housekeeping gene [217].

\begin{tabular}{|l|l|}
\hline Step 1 & 2 minutes at $50^{\circ} \mathrm{C}$ \\
\hline Step 2 & 10 minutes at $95^{\circ} \mathrm{C}$ \\
\hline Step 3 & 5 minutes at $95^{\circ} \mathrm{C}$ for 40 cycles \\
\hline Step 4 & 11 minutes at $60^{\circ} \mathrm{C}$ \\
\hline
\end{tabular}

Table 18. Program used for Quantitative Real-Time PCR (RT- qPCR) using ABI 7500 Fast Real Time PCR System.

\begin{tabular}{|l|l|}
\hline RT-qPCR master mix reagents & Volume $(\mu \mathrm{L})$ \\
\hline TaqMan Gene Assay-FAM & 1 \\
\hline TaqMan Univ PCR MM (2X), w/UNG & 10 \\
\hline $\mathbf{H}_{2} \mathbf{O}$ (nuclease free Water) & 4 \\
\hline
\end{tabular}

Table 19. RT-qPCR master mix composition. 


\begin{tabular}{|l|l|l|}
\hline Gene & NCBI Ref. Seq. & ID Number \\
\hline GAPDH & NM_001256799.2 & Hs02786624_g1 \\
\hline Vimentin & XM_006717500.2 & Hs00958111_m1 \\
\hline E-Cadherin & NM_001317184.1 & Hs01023894_m1 \\
\hline $\boldsymbol{\beta}$-Catenin & NM_001317227.1 & Hs00362037_m1 \\
\hline Twist1 & NM_000474.3 & Hs00361186_m1 \\
\hline
\end{tabular}

Table 20. List of TaqMan assays on demand used (Lifescience Technologies).

\subsubsection{Statistics and data analysis}

Paired t-test was performed to evaluate statistical differences between Hep-Gel and Control-Gel as well as between Hep-Gel Sorafenib or TGF- $\beta 1$ treated and untreated Hep-Gel. The same statistical analysis was performed to evaluate differences upon Sorafenib exposure when cells were culture in 2D plastic surface. SPSS v21 was the software used to perform the statistical analysis 


\subsection{Results}

\section{Assessing the optimal HepG2 cell density in Hep-Gel}

Different concentrations of HepG2 cells were embedded and cultured in Hep-Gel for 13 days in order to define the best cell density through Live/Dead staining analysis.

As shown in Figure 58, different amounts of HepG2 were embedded in Hep-Gel, more specifically $0.33 \times 10^{3}$ cells $/ \mu$ of biomaterial (a), $1.66 \times 10^{3} \mathrm{cells} / \mu \mathrm{l}$ of biomaterial (b), $3.3 \times 10^{3} \mathrm{cells} / \mu \mathrm{l}$ of biomaterial (c) and $5 \times 10^{3}$ cells $/ \mu \mathrm{l}$ of biomaterial (d).

Representative Live/Dead staining images, performed at day 1 of cell culture, showed a homogenous distribution of alive HepG2 (green signal) in the Hep-Gel at all tested cell concentrations. When HepG2 were embedded at higher concentrations, in particular $3.3 \times 10^{3} \mathrm{cells} / \mu \mathrm{l}$, (c) and $5 \times 10^{3} \mathrm{cells} / \mu \mathrm{l}$ (d), Live/Dead images showed that the Hep-Gel were already fully repopulated by HepG2 cells and some cells were found dead, as indicated by the presence of a red signal in the images $(11.6 \%$ dead cells in figure $c$ and $12.6 \%$ dead cells in figure $d$ ). On the contrary, Live/Dead images of HepG2 embedded at lower cell density $(a, b)$ confirmed that the cells were almost all alive (94.5\% alive cells in figure a and $95.9 \%$ in figure b) since only the green signal generated by alive cells was captured in the images. Furthermore, especially at the lowest cell density (a) the Hep-Gel hydrogel was only partially repopulated, allowing the cells to better move and proliferate. Overall, the Live/Dead images captured after 1 day of cell culture suggested that the best HepG2 cell density was $0.33 \times 10^{3}$ cells/ $/ \mathrm{l}$ of Hep-Gel (a). 

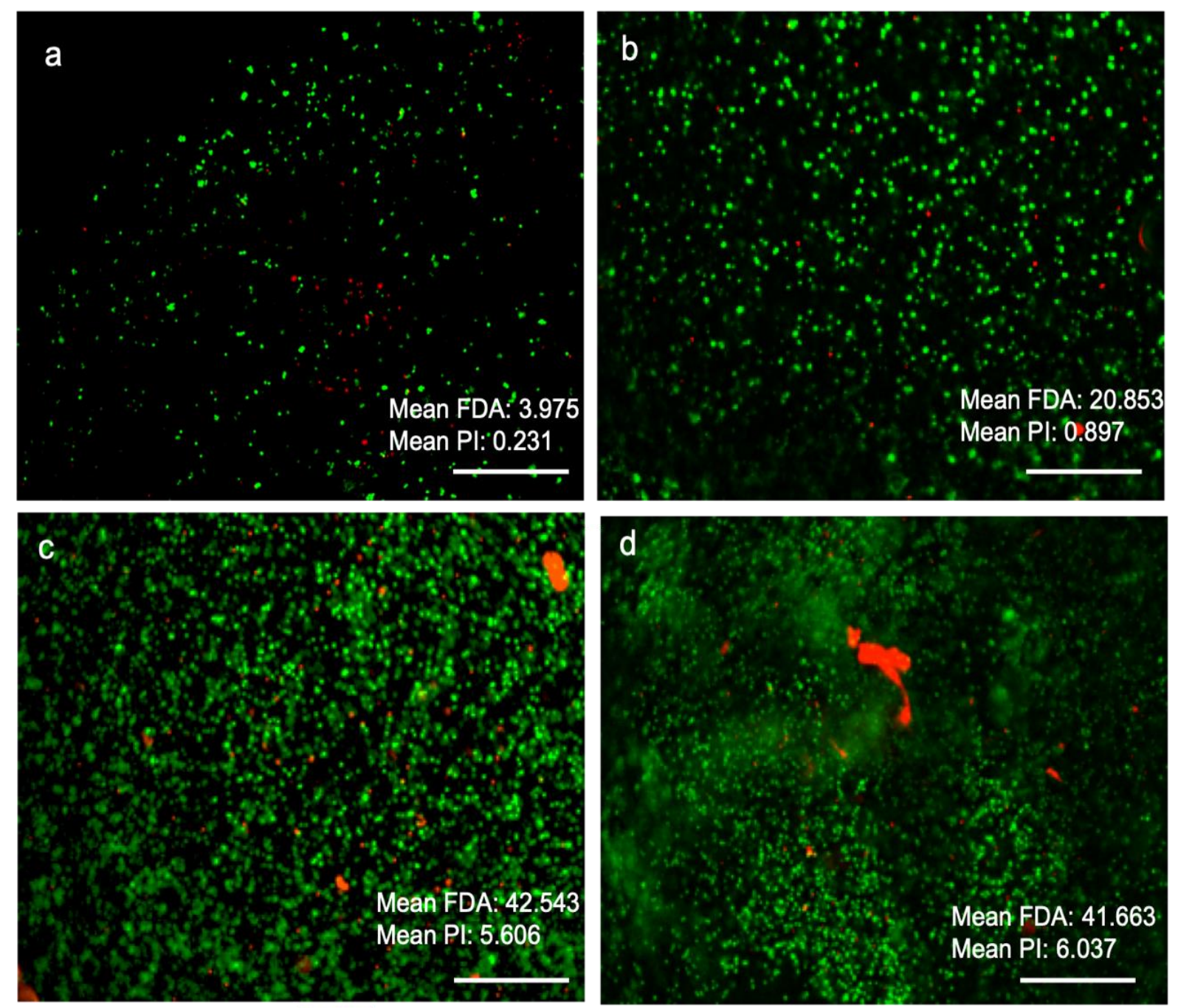

Figure 59. Live/Dead staining of HepG2 embedded in Hep-Gel at different cell density. (a) $0.33 \times 10^{3} \mathrm{cells} / \mu \mathrm{l}$ of biomaterial, (b) $1.6 \times 10^{3} \mathrm{cells} / \mu \mathrm{l}$ of biomaterial, (c) $3.3 \times 10^{3} \mathrm{cells} / \mu \mathrm{l}$ of biomaterial and (d) $5 \times 10^{3}$ cells/ $\mu$ l of biomaterial. Images were captured after 1 day of cell culture. Mean fluorescence intensity for FDA and PI was calculated using Image J. Scale bar (a-d) $500 \mu \mathrm{m}$.

Live/Dead images captured after 13 days of cell culture in Hep-Gel (Figure 59), confirmed that the lowest cell density, $0.33 \times 10^{3} \mathrm{cells} / \mu \mathrm{l}$ of biomaterial (a) was the best in terms of cell viability ( $85.1 \%$ alive cells). Indeed, at this cell concentration and after 13 days of culture all HepG2 were found alive (green signal) while the other tested cell densities (b,c,d) led to a much higher cell death as indicated by the intense red signal observed in the captured Live/Dead images, particularly $72.7 \%$ dead cells in figure $b$, $61.2 \%$ dead cells in fugure $\mathrm{c}$ and $73.1 \%$ in figure $\mathrm{c}$. 
For this reason, $0.33 \times 10^{3}$ cells $/ \mu$ l of biomaterial, corresponding to $0.165 \times 10^{5}$ cells in $50 \mu \mathrm{l}$ of biomaterial, was selected as the optimal cell density, which was used to perform the experiments further described in this Chapter.
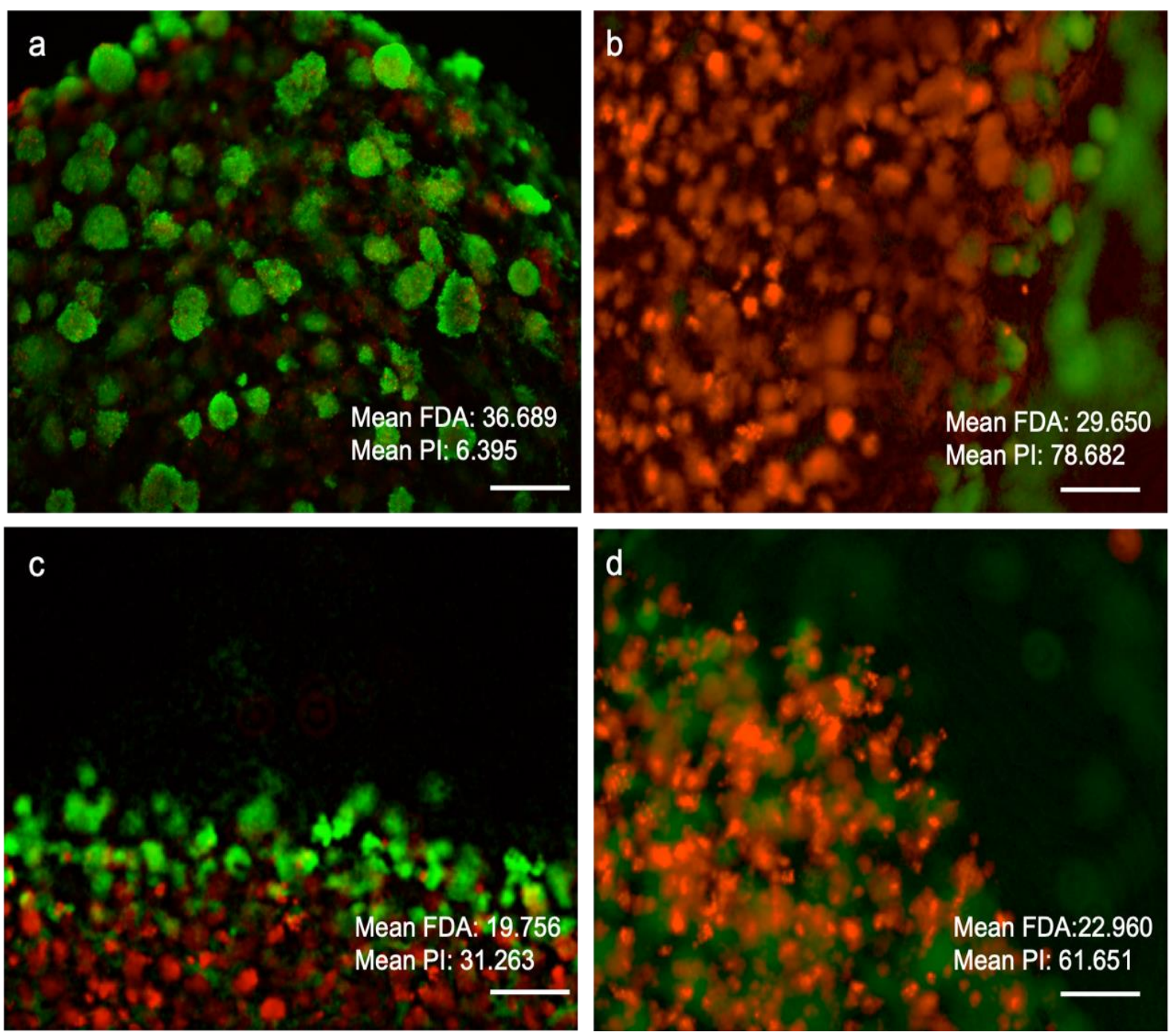

Figure 60. Live/Dead staining of HepG2 embedded in Hep-Gel at different cell density. (a) $0.33 \times 10^{3} \mathrm{cells} / \mu \mathrm{l}$ of biomaterial, (b) $1.6 \times 10^{3} \mathrm{cells} / \mu \mathrm{l}$ of biomaterial, (c) $3.3 \times 10^{3} \mathrm{cells} / \mu \mathrm{l}$ of biomaterial and (d) $5 \times 10^{3}$ cells/ $\mu$ l of biomaterial. Images were captured after 13 day of cell culture. Mean fluorescence intensity for FDA and PI was calculated using Image J. Scale bar (a-d) $500 \mu \mathrm{m}$.

\subsubsection{Assessing the in vitro biocompatibility of the Hep-Gel}

In vitro biocompatibility of the Hep-Gel was investigated by embedding and culturing $0.165 \times 10^{5}$ HepG2 cells in each Hep-Gel (50 $\mu$ l of biomaterial with the optimal cell 
density of $0.33 \times 10^{3}$ cell $/ \mu$ l of biomaterial) for up to 13 days $(n=4)$. Control-Gel was used as internal 3D platform to evaluate if the presence of ECM components would enhance HepG2 cell viability, proliferation and functionality. To do so, similar to the Hep-Gel, $0.165 \times 10^{5} \mathrm{HepG} 2$ were embedded and cultured in each Control-Gel (50 $\mu \mathrm{l}$ of biomaterial with the optimal cell density of $0.33 \times 10^{3} \mathrm{cell} / \mu \mathrm{l}$ of biomaterial) up to 13 days $(n=4)$.

Cell viability and proliferation was investigated by performing Live/Dead staining, Presto Blue assay, histological analysis and immunofluorescence. Functionality of HepG2 cells was evaluated by employing human albumin ELISA assay.

\subsubsection{Live/Dead staining}

Live/Dead staining of HepG2 cells embedded in Hep-Gel or Control-Gel was performed at day 1 (Figure 60 a-d), day 7 (Figure 60 e-h) and day 13 (Figure i-n).

As shown in the representative Live/Dead images, after 1 day of cell culture, HepG2 cultured in both Hep-Gel (a,b) and Control-Gel (c,d) were all alive (93\% alive cells in both conditions), as indicated by the presence of the green signal and the absence of red signal, and showed to be homogeneously distributed into the hydrogels and organised in single cells or small clusters of 2-4 cells.

After 7 days of cell culture, HepG2 cultured in Hep-Gel (e,f) were found essentially all alive (95.3\% alive cells), as indicated by the high intensity of the green signal generated by the alive cells and the low intensity of red colour generated by dead cells. Moreover, at this time point, captured Live/Dead images showed that HepG2 were organised in spheroids of around $100 \mu \mathrm{m}$ in diameter and very close to each other especially at the border of the hydrogels.

When analysing the Control-Gel $(\mathrm{g}, \mathrm{h})$, HepG2 cells appeared to be mainly alive but the image captured at higher magnification $(h)$ showed that some cells were dead (13.3\% dead cells), as indicated by the presence of red spots in the aggregates located on the top left part of the picture. Similar to Hep-Gel condition, at this time point HepG2 cultured in Control-Gel were organised in spheroids of around $100 \mu \mathrm{m}$ in 
diameter but spheroids did not seem to be in contact with each other indicating a possible inhibition in cell proliferation.

After day 13 of cell culture, HepG2 cultured in Hep-Gel (i,l) were still all alive (97\% alive cells) and repopulated the whole hydrogels, as indicated by the intense green signal in Figures $\mathrm{i}$ and I. Furthermore, the diameter of the HepG2 spheroids increased up to around $300 \mu \mathrm{m}$, in comparison to $100 \mu \mathrm{m}$ diameter at day 7 (Figures e,f for day 7 and i,l for day 13 of cell culture), suggesting a massive cell proliferation. Important, the image captured at high magnification (I) did not show any sign of HepG2 cell death in the middle of the spheroids, confirming a great efficiency in oxygen and nutrient diffusion throughout the Hep-Gel. On the contrary, HepG2 cells cultured for 13 days in Control-Gel $(m, n)$ were found more dead than alive (55.7\% dead cells), since a more intense red signal was detected derived from dead cells compared to the green signal originated from alive cells. Furthermore, the size of the spheroids slightly increased compared to day 7 , suggesting a limited cell growth in the Control-Gel.

Overall, the captured Live/Dead images confirmed the superiority of Hep-Gel in supporting HepG2 cell viability and proliferation during the in vitro culture in comparison to Control-Gel. 

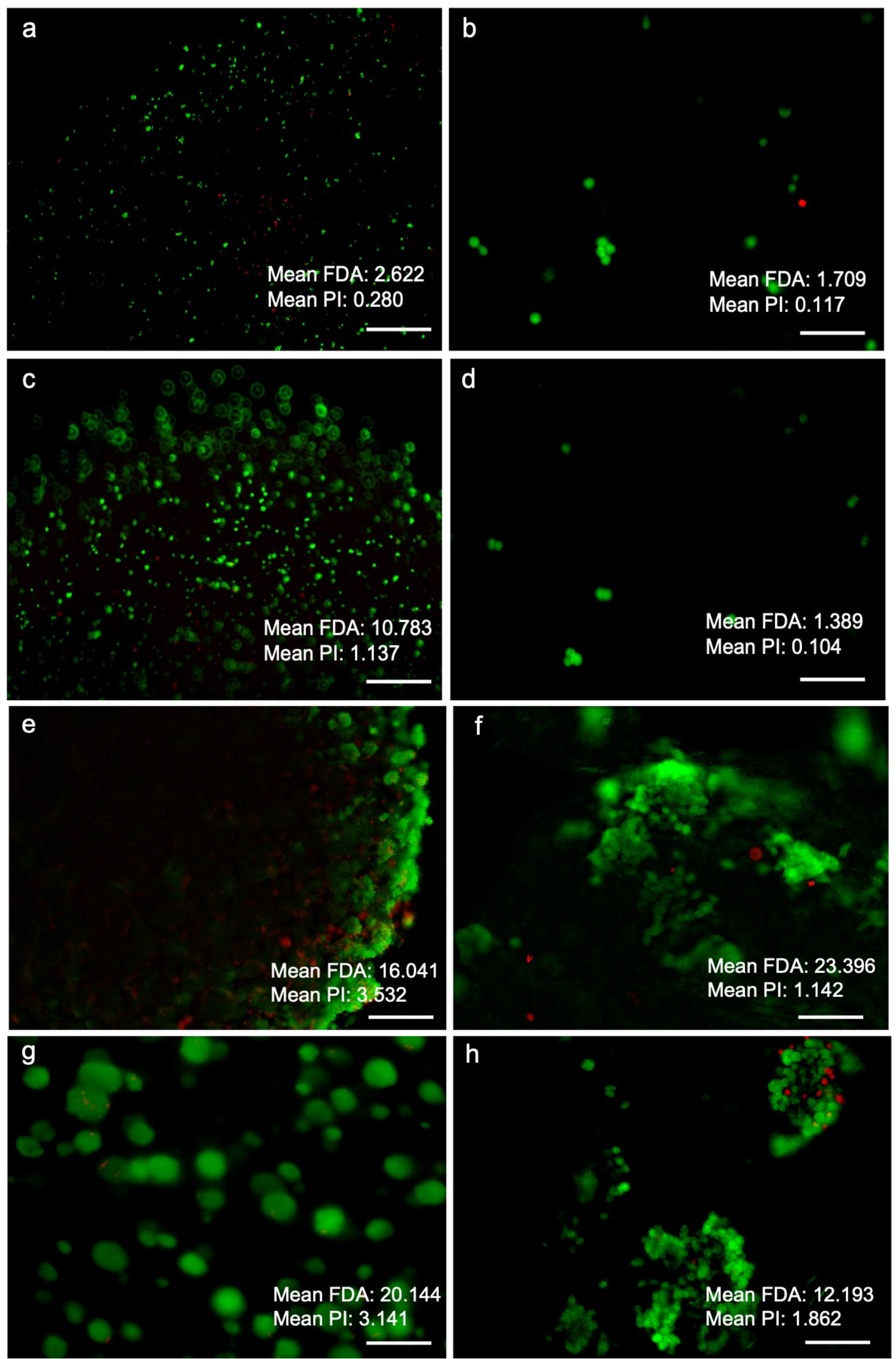

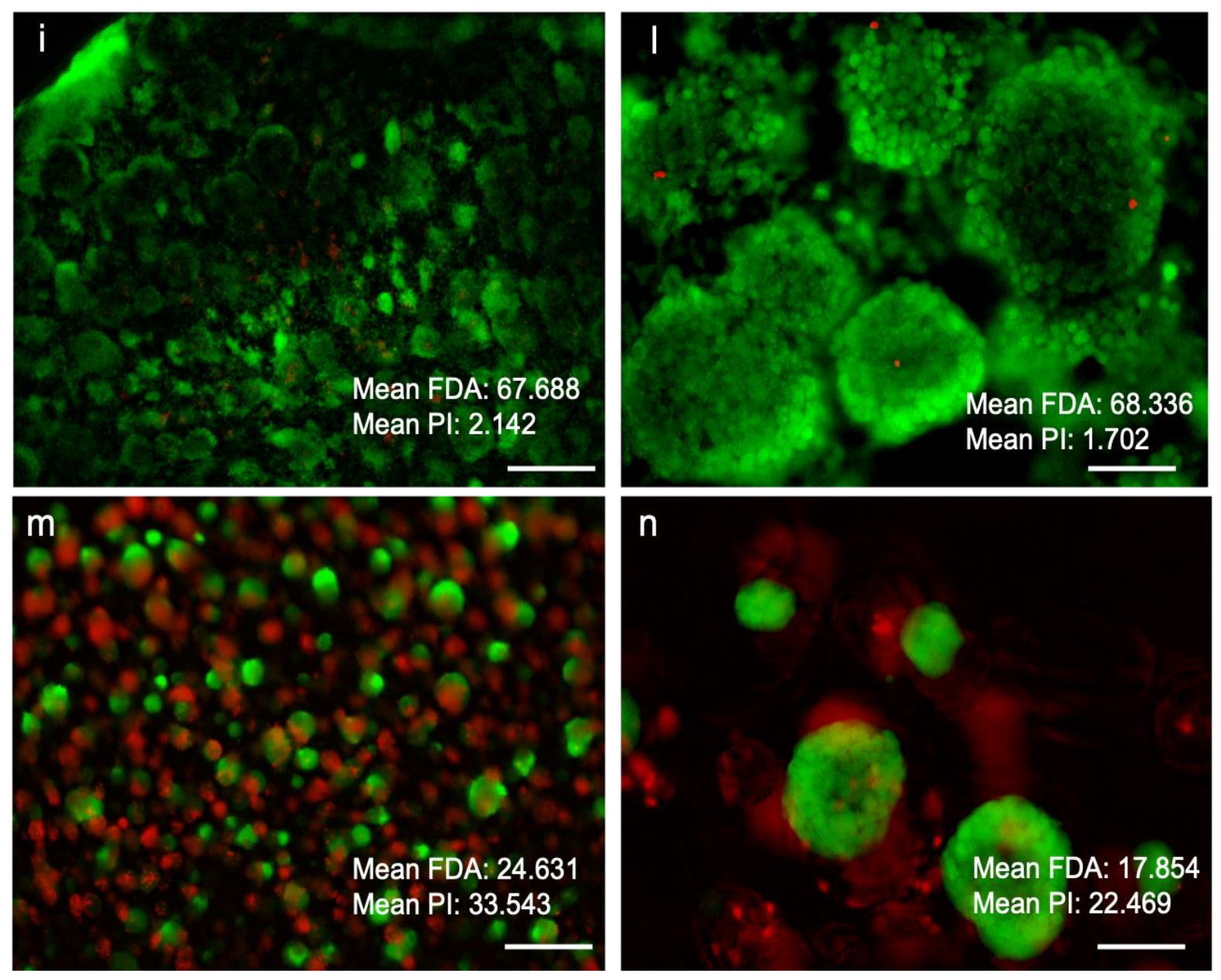

Figure 61. Live/Dead staining of HepG2 cells cultured in Hep-Gel (a,b,e,f,i,l) and Control-Gel (c,d,g,h,n,m) at day 1 (a-d), day 7 (e-h) and day 13 (i-n) of cell culture. Mean fluorescence intensity for FDA and PI was calculated using Image J. Scale bar (a,c,e,g,i,m) $500 \mu \mathrm{m}$, (b,d,f,h,l,n) $100 \mu \mathrm{m}$.

\subsubsection{Presto blue assay}

Presto blue assay was performed to assess the metabolic activity and proliferation rate of HepG2 cultured in Hep-Gel or Control-Gel at different time points during the cell culture (day 1 , day 7 and day 13).

As shown in Figure 61, HepG2 cultured in both Hep-Gel and Control-Gel showed an increased trend in metabolic activity and proliferation correlated with the days of culture. Notably, HepG2 showed a significantly higher metabolic profile in Hep-Gel compared to Hep-Gel or Control-Gel at both 1 day $\left({ }^{* *}, \mathrm{P}<0.001\right)$ and 7 days $\left({ }^{* *}\right.$, $\mathrm{P}<0.01$ ) of cell culture. Results obtained after 13 days of cell culture did not show any difference in metabolic activity between the two conditions. This was due to the higher 
number of HepG2 cells in the samples that cause a saturation, meaning that linearity of the signal has reached a plateau. These data therefore do not rule out possible differences between cell proliferation and metabolic activity between the conditions tested. In another way this result confirmed that in both Hep-Gel and Control-Gel, after 13 days of cell culture, there was an increased HepG2 number and metabolic activity compared to day 1 and 7 .

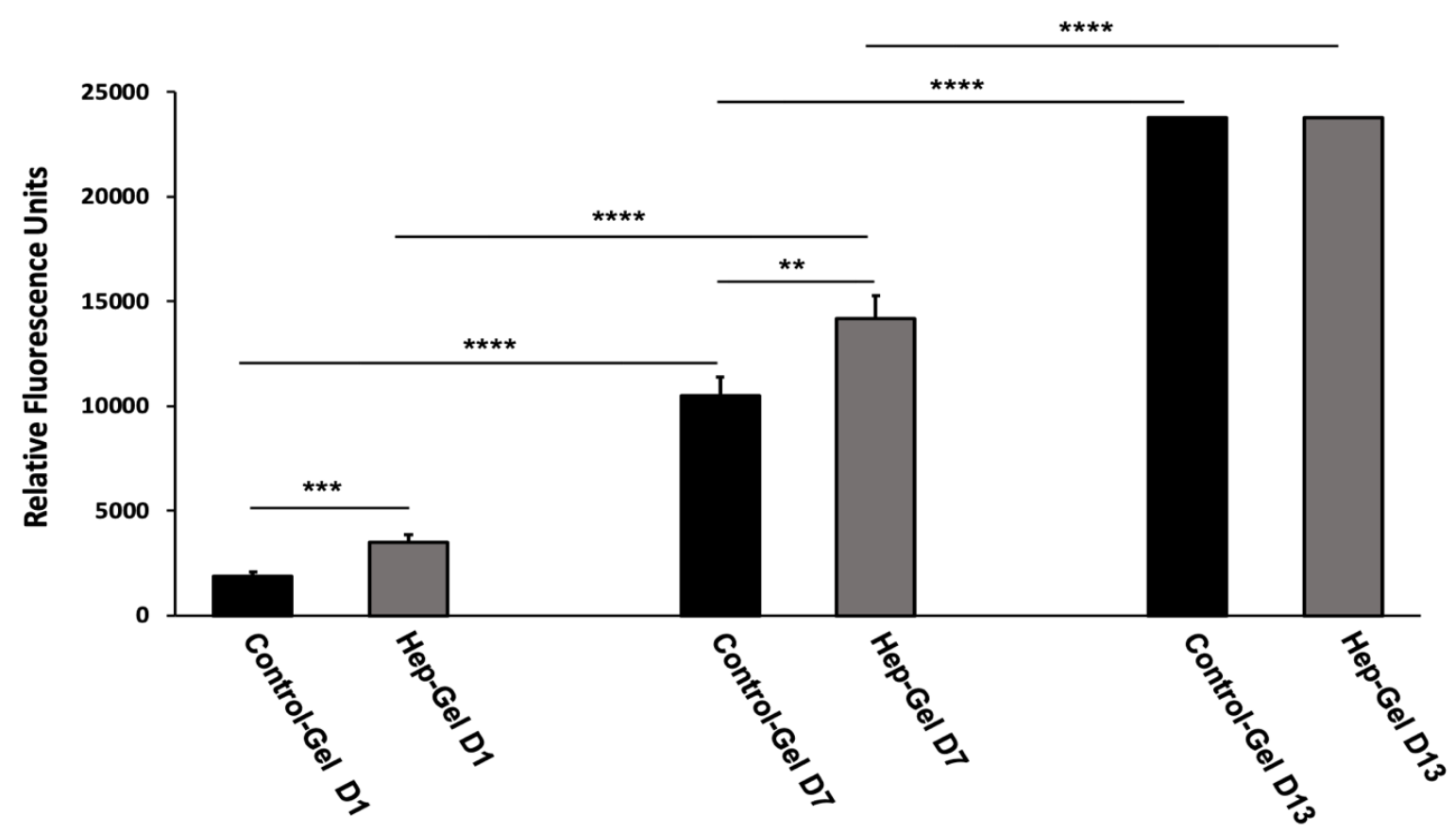

Figure 62. Presto blue assay performed to investigate metabolic activity and proliferation of HepG2 cells cultured in Hep-Gel and Control-Gel at day 1 (a), day 7 (b) and day 13 (c) of cell culture. ${ }^{* *} p<0.01,{ }^{* * *} p<0.001,{ }^{* * *} p<0.0001 . n=4$ per condition.

\subsubsection{Histological analysis}

Haematoxylin and Eosin staining and Sirius Red staining of bioengineered Hep-Gel and Control-Gel with HepG2 were performed after 7 and 13 days of cell culture.

Practical limitations were present when handling bioengineered Control-Gel with HepG2. These gels were very fragile and difficult to handle, as it was impossible to move them from the cell culture plate to a new plate for the fixation process without affecting their integrity. Furthermore, only a small portion of the Control-Gel remained intact after fixation in $10 \%$ formalin and dehydration cycles in Industrial Denatured 
Alcohol (IDA) and xylene baths prior the embedding in paraffin wax and histological staining.

In contrast, bioengineered Hep-Gel with HepG2 were easy to handle and were not affected by the fixation process or the dehydration cycles in Industrial Denatured Alcohol (IDA) (Acquascience) and xylene. For this reason, in case of Hep-Gel the entire samples were analysed.

Haematoxylin and Eosin staining (Figure 62 a,b) and Sirius Red staining (Figure 62 c,d) of HepG2 cultured for 7 days in Control-Gel showed that the cells were alive, as confirmed by the blue stained nuclei in the Haematoxylin and Eosin images (a,b), and cells were organised in spheroids of $50-100 \mu \mathrm{m}$ in diameter. 


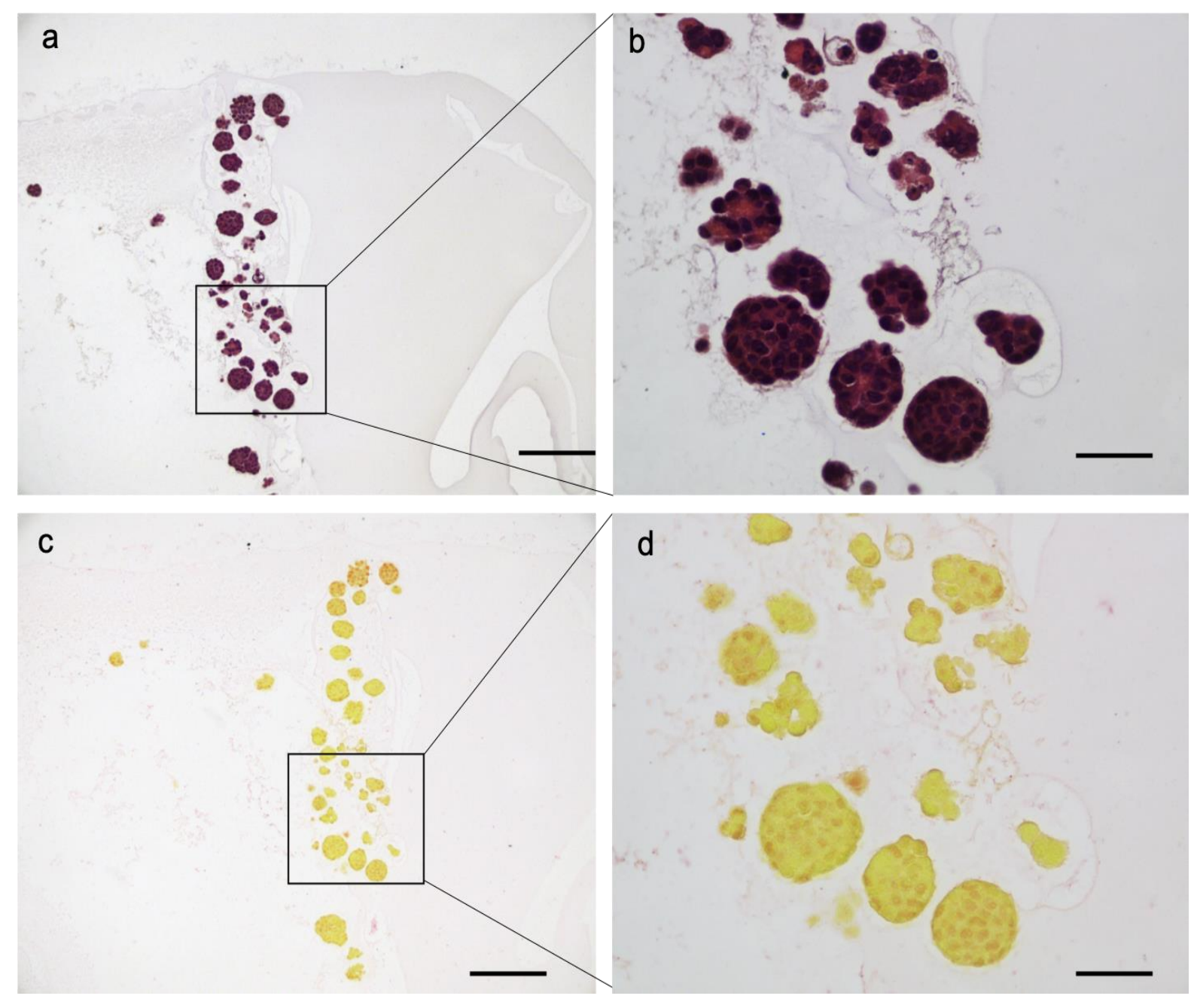

Figure 63. Haematoxylin and eosin staining (a,b) and Sirius Red staining (c,d) of HepG2 cells cultured for 7 days in Control-Gel. Scale bar (a,c) $200 \mu \mathrm{m}$, (b,d) $50 \mu \mathrm{m}$.

A similar result was obtained for HepG2 cells cultured for 13 days in Control-Gel (Figure $63 \mathrm{e}-\mathrm{h}$ ), a slight increase in the spheroid diameter was detected compared to samples analysed after 7 days of culture. Furthermore, the Haematoxylin and Eosin image captured at higher magnification (f), showed that especially in the bigger spheroids, several nuclei were not stained in blue by the eosin dye, suggesting that these cells were dead. These histological results confirmed those obtained through the Live/Dead staining regarding the cell death and the low growth rate of HepG2 cells in the Control-Gel at day 7. 


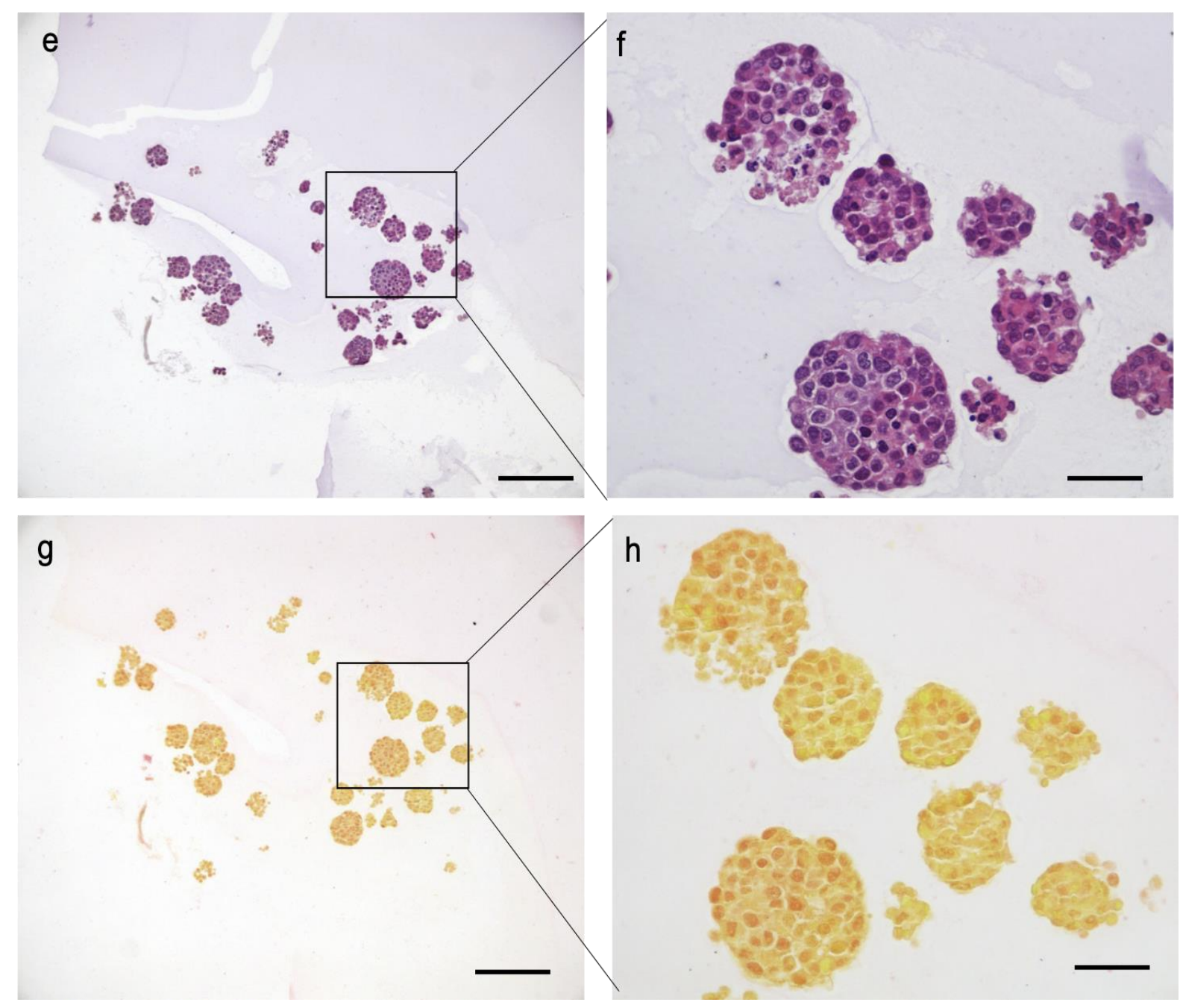

Figure 64. Haematoxylin and eosin staining (e,f) and Sirius Red staining (g,h) of HepG2 cells cultured for and 13 days in Control-Gel. Scale bar $(e, g) 200 \mu \mathrm{m},(\mathrm{f}, \mathrm{h}) 50 \mu \mathrm{m}$.

Haematoxylin and Eosin (Figure 64 a,b) and Sirius Red images (Figure 64 c,d) of HepG2 cultured for 7 days in Hep-Gel showed that the cells were alive, as confirmed by the blue stained nuclei in the Haematoxylin and Eosin images $(a, b)$ and were organised in spheroids of $100 \mu \mathrm{m}$ in diameter. According to Live/Dead results, the majority of HepG2 spheroids were found on the external part of the hydrogels and seem to be very close or even in contact with each other. Furthermore, these histological staining confirmed that in all areas of the hydrogels there was an homogeneous distribution of ECM components, as indicated by the pink colour in Haematoxylin and Eosin $(a, b)$ and accumulation of red colour in the Sirius Res images $(c, d)$. 


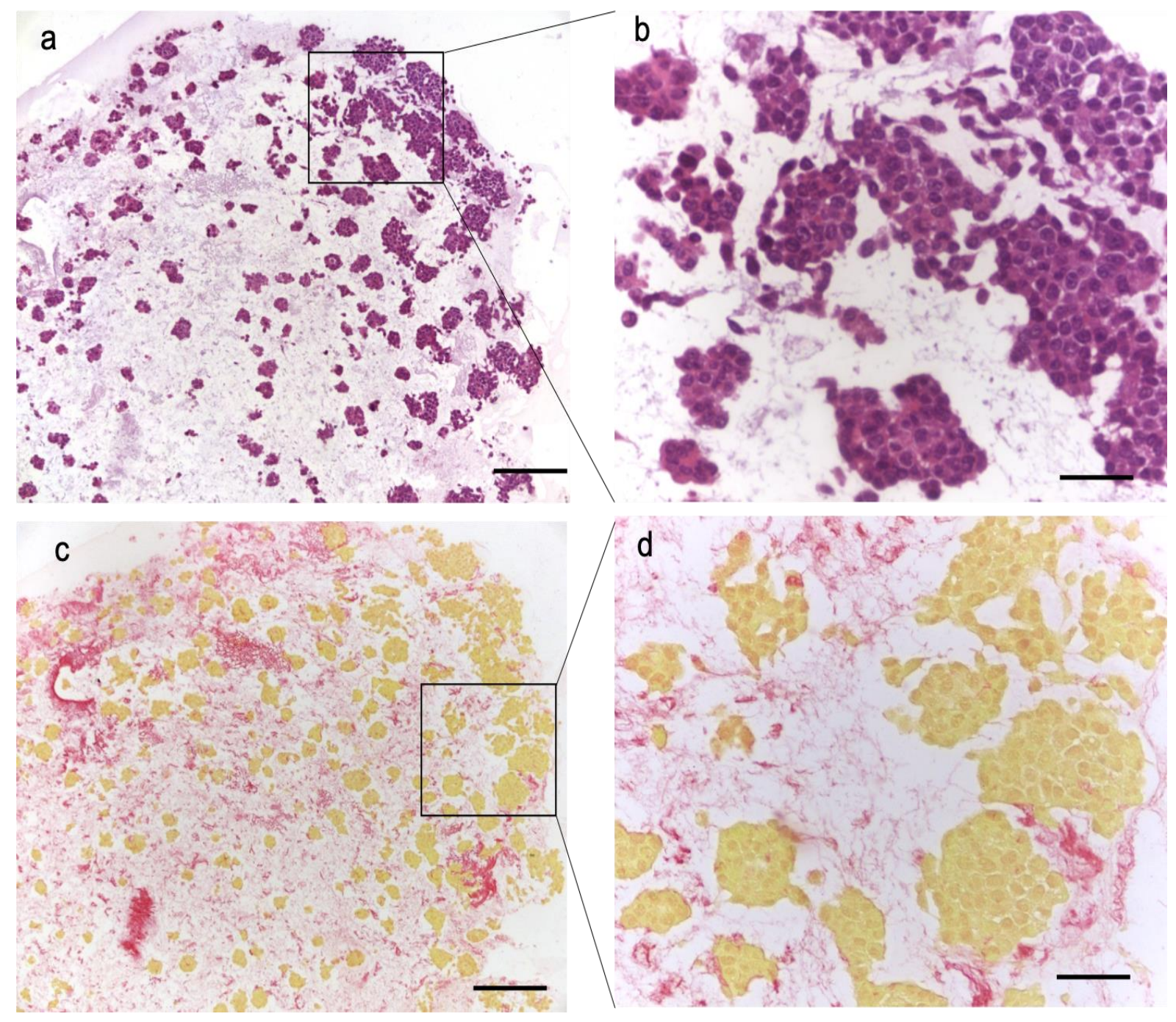

Figure 65. Haematoxylin and eosin staining (a,b) and Sirius Red staining (c,d) of HepG2 cells cultured for 7 days in Hep-Gel. Scale bar (a,c) $200 \mu \mathrm{m}$, (b,d) $50 \mu \mathrm{m}$.

Haematoxylin and Eosin (Figure 65 a,b) and Sirius Red images (Figure 65 c,d) of HepG2 cultured for 13 days in Hep-Gel, confirmed that cells where all alive and repopulated the entire hydrogels. Compared to the samples fixed after 7 days of cell culture, samples fixed after 13 days of cell culture showed an increased number and size of the spheroids, confirming the fast cell growth in these hydrogels composed of ECM components.

According to the previous results obtained by employing Live/Dead staining and Presto Blue assay, the histological analysis confirmed that the presence of ECM components enhanced HepG2 viability and proliferation. 


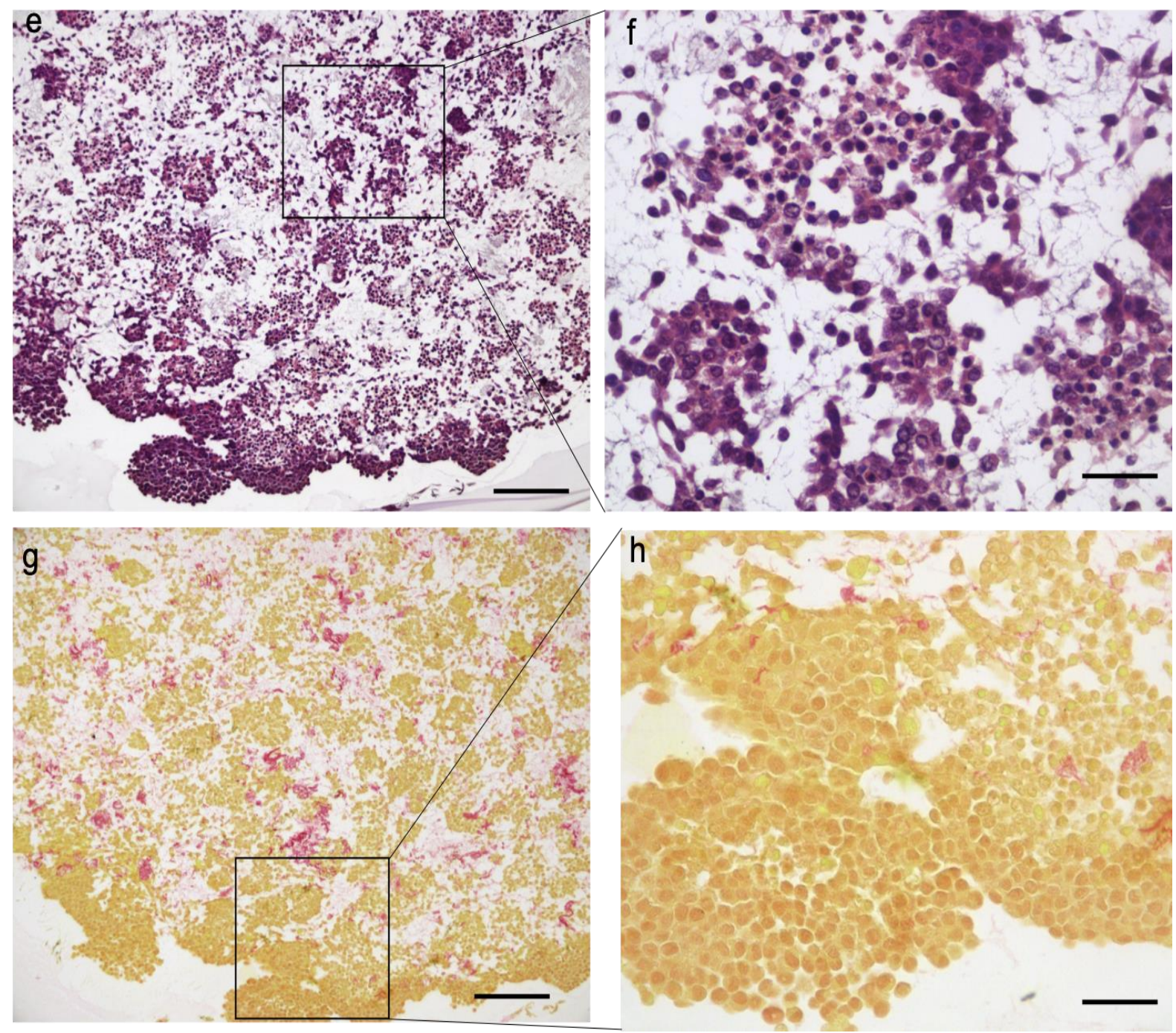

Figure 66. Haematoxylin and eosin (e,f) and Sirius Red staining (g,h) of HepG2 cells cultured for 13 days in Hep-Gel. Scale bar (e,g) $200 \mu \mathrm{m}$, (f,h) $50 \mu \mathrm{m}$.

\subsubsection{Immunofluorescence}

Immunofluorescence staining of HepG2 cultured for 13 day in both Hep-Gel and Control-Gel was performed with fluorescent Hoechst to stain the nuclei and fluorescent Phalloidin staining to capture the structure of the F-actin cytoskeleton.

As shown in Figure 66, merged Hoechst and Phalloidin images showed that HepG2 cultured in both Hep-Gel (a,b) and Control-Gel (c,d) maintained a round shape typical of epithelial cells. Important, the image captured at higher magnification for Hep-Gel (b) showed that all nuclei, including those in the bigger spheroid, were stained by the Hoechst, confirming that cells were alive. This also confirmed the efficient diffusion of 
nutrients and oxygen throughout the hydrogel. Moreover, the images captured at higher magnification $(b, d)$ confirmed the bigger size of HepG2 spheroids in Hep-Gel (b) compared to Control-Gel (d).
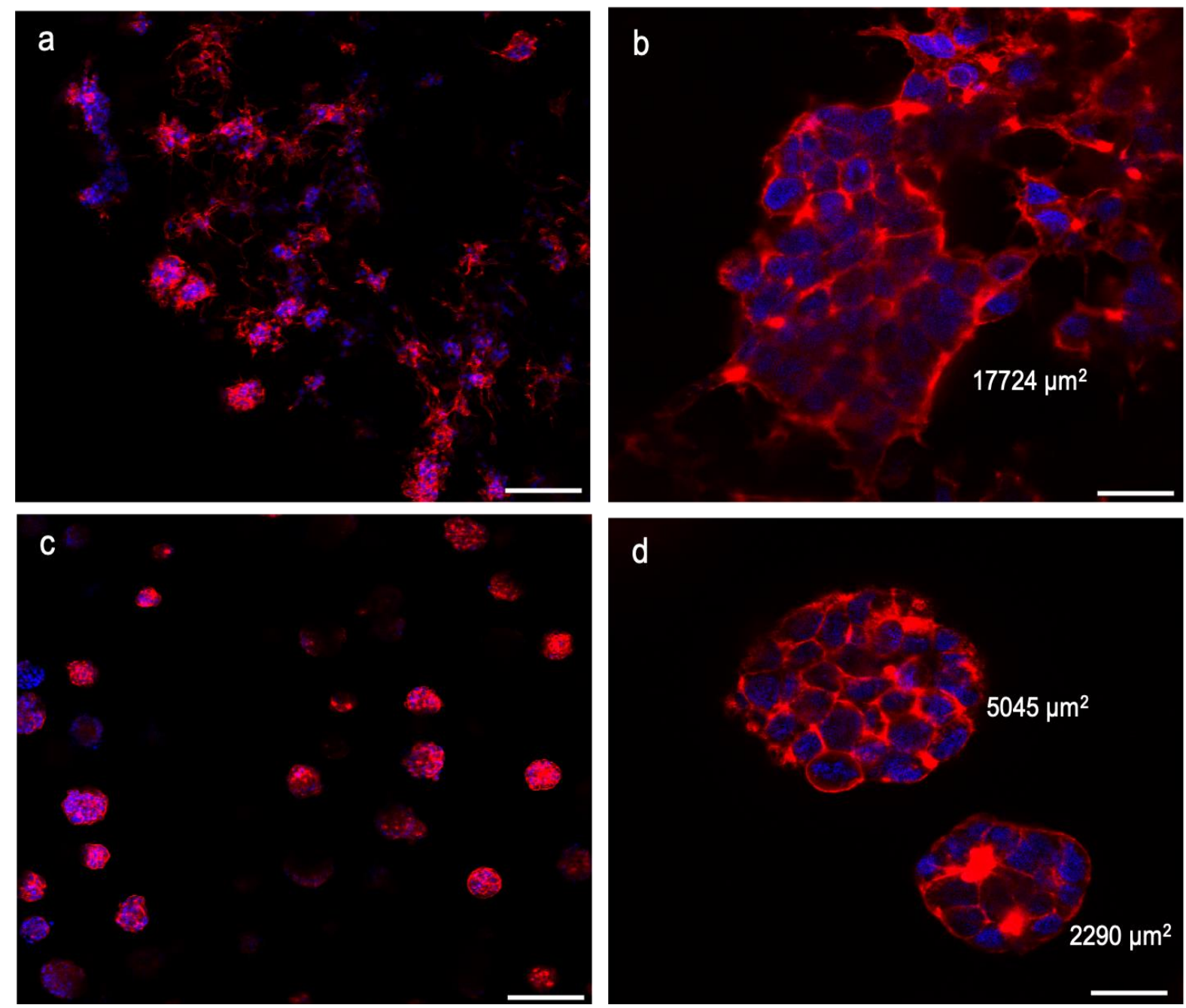

Figure 67. Merged fluorescent Phalloidin with Hoechst staining of HepG2 cells cultured for 13 days in Hep-Gel $(a, b)$ and Control-Gel $(c, d)$. Area of the clusters was quantified using Image J. Scale bar $(a, c) 200 \mu \mathrm{m},(b, d) 30 \mu \mathrm{m}$.

Immunofluorescence staining with Hoechst I and epithelial cell adhesion molecule (EpCAM) antibody was performed to further confirm that HepG2 maintained their natural epithelial phenotype when cultured in Hep-Gel.

EpCAM is a glycoprotein located on the plasma membrane of epithelial cells, for cellcell adhesion [410]. 
Representative confocal images (Figure 67 a-c) showed that HepG2 cultured in HepGel were positive to Hoechst staining (a) and EpCAM staining (b), and confirming the preservation of their natural epithelial phenotype. Furthermore, the high expression of this important protein for cell-cell adhesion from HepG2 cells cultured in Hep-Gel, justified their rapidly proliferating behaviour and their ability to create spheroids rather than remain in single cells.
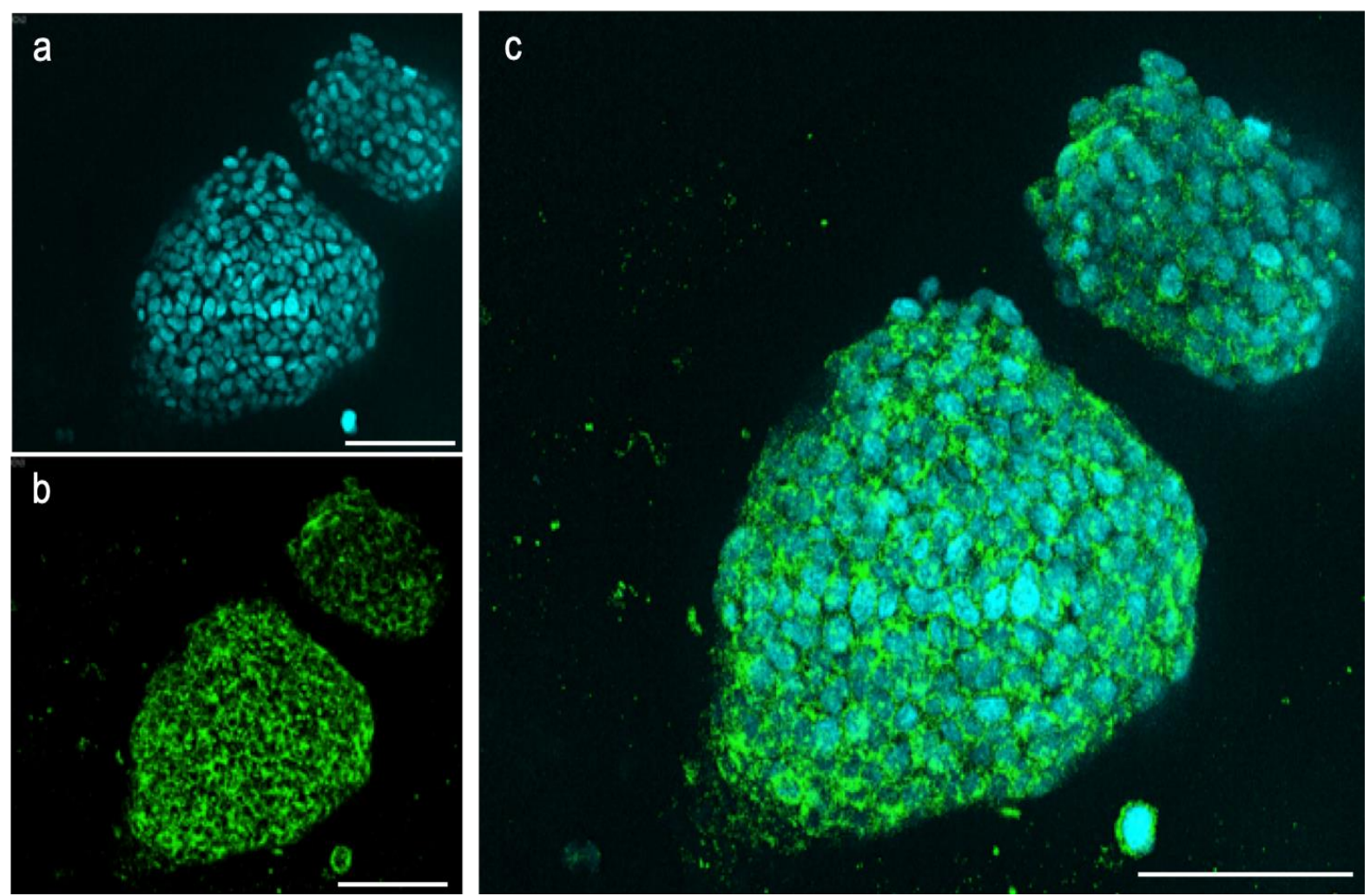

Figure 68. (a) Fluorescent Hoechst staining, (b) EpCAM antibody staining and (c) merged DAPI and EpCAM staining of HepG2 cultured for 13 days in Hep-Gel. Scale bar (a-c) $100 \mu \mathrm{m}$.

\subsubsection{Human albumin ELISA assay}

Human Albumin ELISA assay was carried out to investigate the metabolic activity and functionality of HepG2 cultured for 13 days in Hep-Gel and Control-Gel.

As shown in Figure 68, the presence of ECM components improved functionality and metabolic activity of HepG2, as suggested by the increased secretion of albumin from Hep-Gel condition compared to Control-Gel condition $\left.{ }^{* * * *}, \mathrm{P}<0.0001\right)$. 
Overall, all the data generated so far i.e. including those obtained through the Live/Dead staining, presto blue assay, histology, immunohistochemistry and human albumin ELISA assay, showed that HepG2's viability, proliferation, metabolic activity and functionality is dramatically enhanced when cells are cultured in the hydrogels composed of ECM components. For this reason, Hep-Gel, rather than Control-gel, was chosen in the following set of experiments further described in this Chapter.

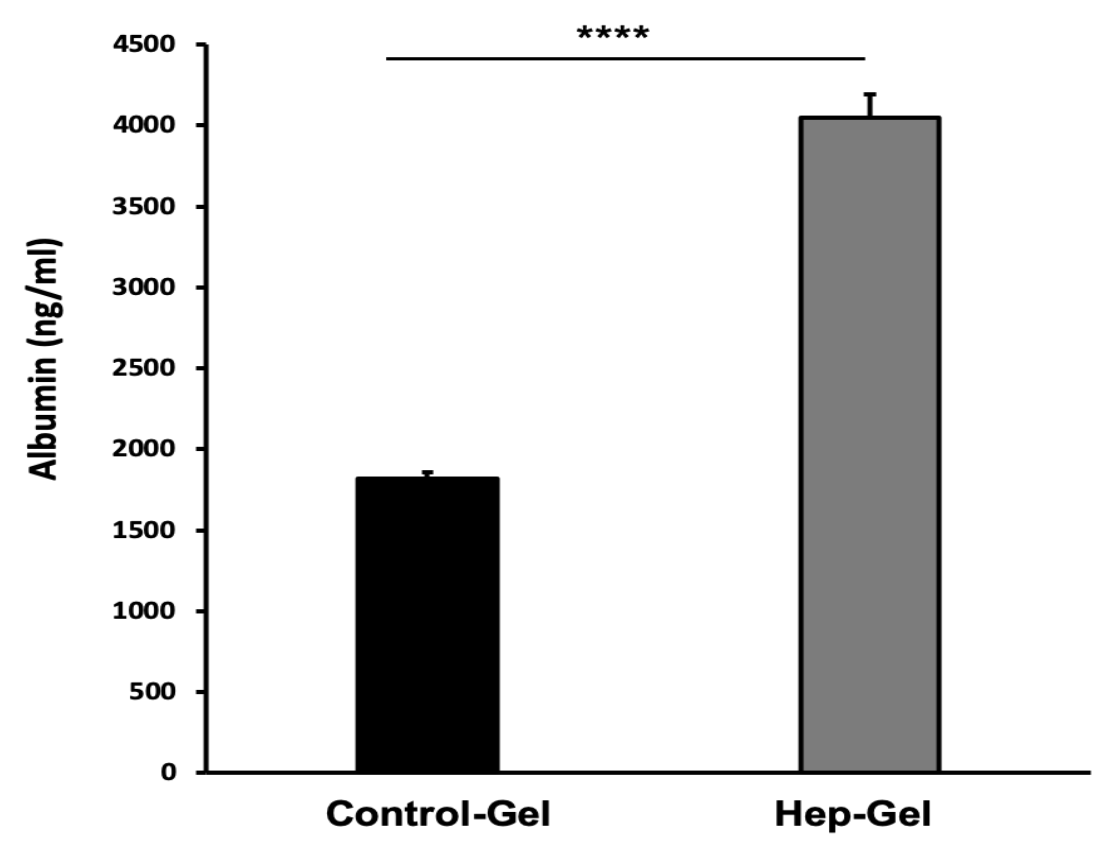

Figure 69. HepG2 albumin secretion from Hep-Gel and Control-Gel after 13 days of cell culture. ${ }^{* * *} p<0.0001 . n=4$ per condition.

\section{In-vitro Hepatocellular Carcinoma (HCC) models}

\subsubsection{Sorafenib treatment in 2D plastic culture}

HepG2 and SNU-449 cell lines were plated in 2D culture system (12-well plates) at a density of $3 \times 10^{5}$ cells per well ( $n=4$ per condition). After 1 day of $2 \mathrm{D}$ cell culture both cells reached the optimal $70 \%$ cell density and Sorafenib $2.5 \mu \mathrm{M}$ was applied to treat the samples for 48 hours. Changes in cell viability caused by this selected chemo drug, was assessed through Presto Blue assay and bright-field microscopy images. 
Bright-field microscopy images (Figure 69 a,b) showed that untreated HepG2 cells (a) were all alive and reached almost $100 \%$ of cell confluency. In contrast, Sorafenib treatment (b) induced cell death HepG2, as confirmed by the fact that all HepG2 cells were found detached. The few cells captured in the representative image (b) were dead and floating in the culture medium.

The Presto blue assay results (Figure $69 \mathrm{c}$ ) also confirmed the complete Sorafenibinduced cell death at $2.5 \mu \mathrm{M}$ for 48 hours $\left.{ }^{* * \star *}, \mathrm{P}<0.0001\right)$ since no colour development and no absorbance signal was detected in the case of Sorafenib treated samples.
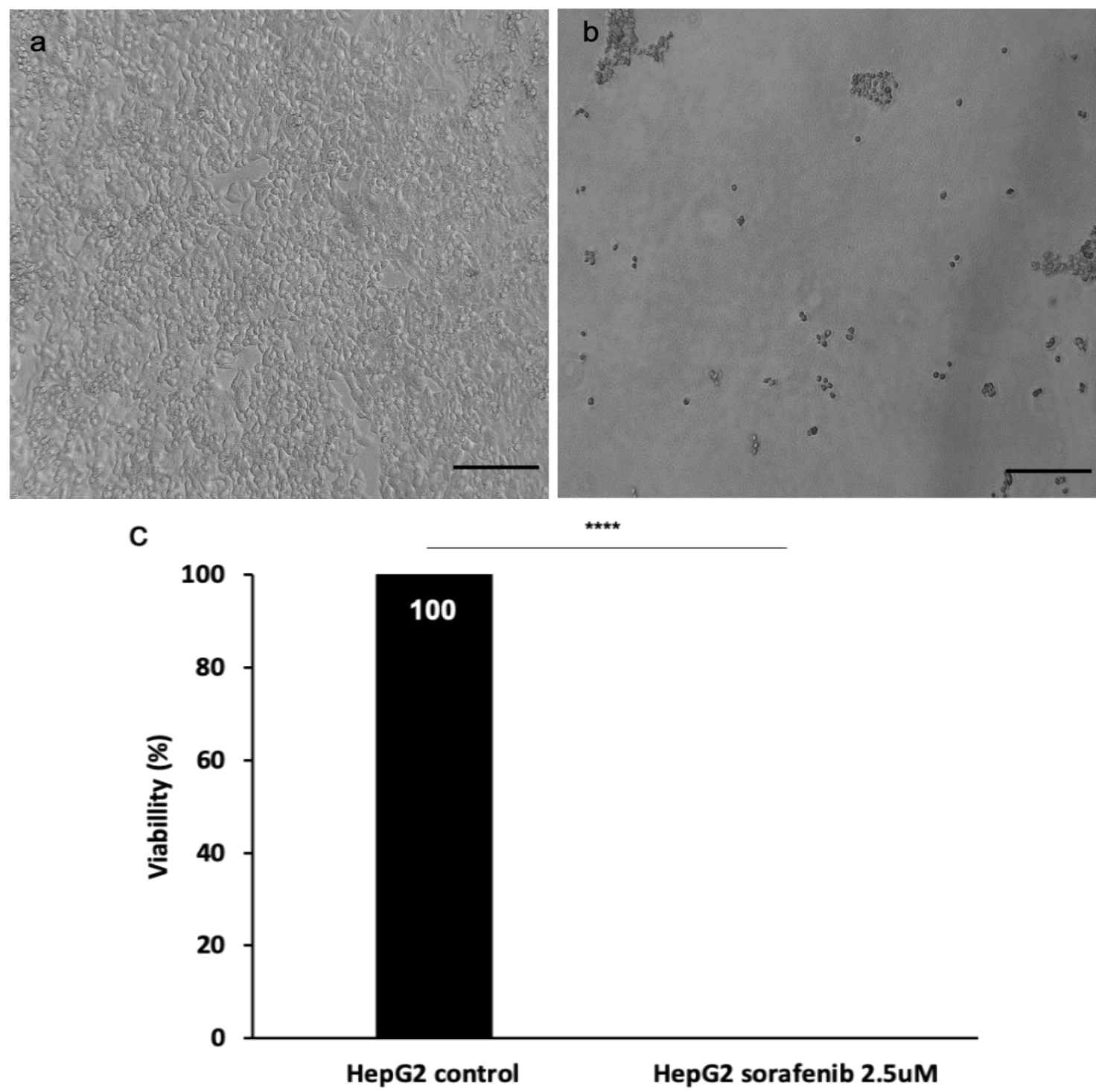

Figure 70. Bright-field microscopy images of (a) HepG2 control samples and (b) HepG2 treated samples with Sorafenib $2.5 \mu \mathrm{M}$ for 48 hours. (c) Presto blue viability test of treated and untreated HepG2 samples. Scale bar $(a, b) 200 \mu m$. (c) ${ }^{* * * *} p<0.0001 . n=4$ per condition. 
When culturing and exposing SNU-449 cells to Sorafenib, they exhibited a higher chemoresistance to Sorafenib treatment in comparison to HepG2 cells when cultured in the same 2D plastic cell culture system. Bright-field microscopy images (Figure 70 a,b) showed that untreated SNU-449 cells (a) were all alive and reached $100 \%$ of confluency, while Sorafenib treatment caused a reduction in cell viability, since in the captured image (b) it was possible to distinguish spaces not covered by SNU-449 cell.

When employing the Presto Blue viability assay (Figure 670 c) a $48.6 \%$ reduction in SNU-449 cell viability induced by Sorafenib $(2.5 \mu \mathrm{M}, 48$ hours $)$ was quantified compared to untreated samples $\left({ }^{* * *}, \mathrm{P}<0.0001\right)$.
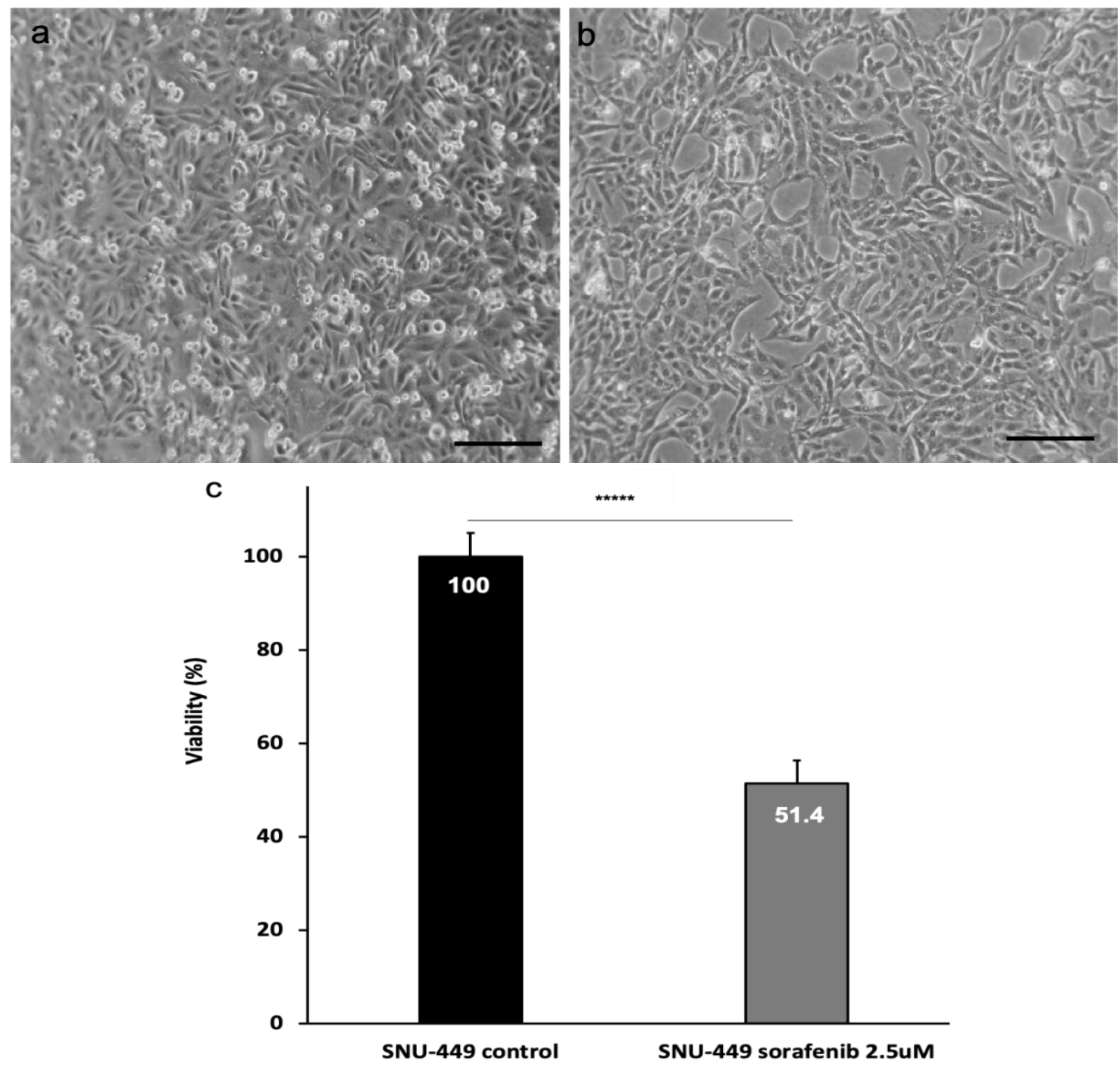

Figure 71. Bright-field microscopy images of (a) SNU-449 control samples and (b) SNU-449 treated samples with Sorafenib $2.5 \mu \mathrm{M}$ for 48 hours. (c) Presto blue viability test of treated and untreated SNU-449 samples. Scale bar $(a, b) 200 \mu \mathrm{m}$. (c) ${ }^{* * * *} p<0.0001$. $n=4$ per condition. 


\subsubsection{Sorafenib treatment in Hep-Gel 3D culture}

The efficiency of Sorafenib in inducing cell death in HepG2 and SNU-449 cells was further evaluated when both cell lines were cultured in Hep-Gel, a more physiologic 3D environment compared to 2D plastic. To do this, HepG2 or SNU-449 were embedded and cultured in Hep-Gel for 7 days prior the treatment with Sorafenib at different concentrations $(2.5 \mu \mathrm{M}, 10 \mu \mathrm{M}$ and $40 \mu \mathrm{M})$ for 48 hours. The clinical dose of $400 \mathrm{mg}$ used in patients were applied to the hydrogels which is equivalent to $10 \mu \mathrm{M}$ [411]. Also, Sorafenib $40 \mu \mathrm{M}$ was used to evaluate a possible resistance of the HepG2 and SNU-449 cell lines towards this kinase inhibitor. Changes in cell viability caused by Sorafenib treatment, were assessed through Presto Blue assay and Live/Dead staining.

Figure 71 showed a decrease in HepG2 cell survival upon Sorafenib treatment based on the results obtained from Live/Dead staining images (a-d) and Presto Blue assay (e). Captured Live/Dead images for untreated control HepG2 samples (a) confirmed that all cells were alive (90\% alive cells), while Sorafenib $2.5 \mu \mathrm{M}$ treatment (b) caused a little increase in cell death but overall HepG2 cells were found much more alive than dead (79.3\% alive cells). Images captured for HepG2 samples treated with Sorafenib $10 \mu \mathrm{M}$ (c) and $40 \mu \mathrm{M}$ (d) showed an equal number in alive and dead cells (56.2\% alive cells in $\mathrm{C}$ and $50.3 \%$ alive cells in d). Important, image captured for Sorafenib $10 \mu \mathrm{M}$ samples demonstrated that the drug was able to reach the middle of the spheroids and to cause the death of several HepG2 cells.

Presto Blue assay results (e) showed a Sorafenib dose dependent decrease in cell viability. Indeed, compared to the untreated control samples, in Sorafenib $2.5 \mu \mathrm{M}$ treated samples HepG2 were found $77 \%$ alive $\left(^{* * *}, \mathrm{P}<0.001\right)$, in Sorafenib $10 \mu \mathrm{M}$ treated samples cells were found $58 \%$ alive $\left({ }^{* \star * *}, \mathrm{P}<0.0001\right)$, while in Sorafenib $40 \mu \mathrm{M}$ treated samples HepG2 cells were found $51 \%$ alive $\left(^{* * * *}, \mathrm{P}<0.0001\right)$. Similar to Live/Dead staining results, Sorafenib $2.5 \mu \mathrm{M}$ caused only a slightly reduction in cell viability, while the clinical dose $10 \mu \mathrm{M}$ and the toxic dose $40 \mu \mathrm{M}$ reduced considerably more the HepG2 cell viability. A statistically significant reduction in HepG2 cell viability was observed while increasing Sorafenib dose from $2.5 \mu \mathrm{M}$ to $10 \mu \mathrm{M}$ ( $\left.{ }^{\star \star \star}, \mathrm{P}<0.0001\right)$ as well as from $10 \mu \mathrm{M}$ to $40 \mu \mathrm{M}\left({ }^{* *}, \mathrm{P}<0.01\right)$. Compared to the conventional $2 \mathrm{D}$ plastic culture system, where HepG2 were found all dead upon treatment with Sorafenib 2.5 
$\mu \mathrm{M}$, HepG2 cultured in Hep-Gel showed to be much more resistant to the chemo treatment.
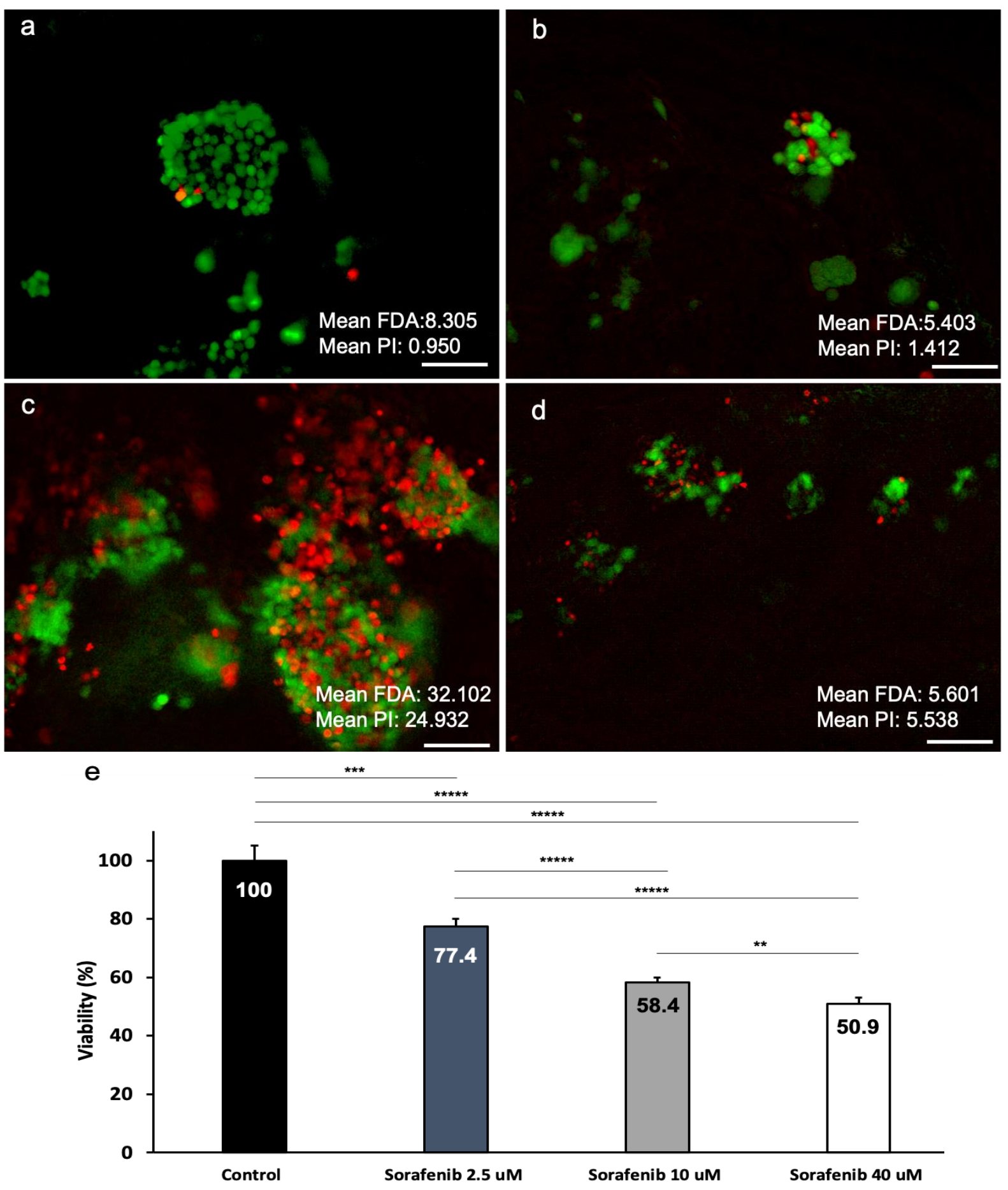

$\star \star \star *$

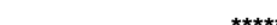

Figure 72. Live/Dead staining images (a-d) of (a) HepG2 untreated samples, (b) HepG2 samples treated with Sorafenib $2.5 \mu \mathrm{M}$, (c) HepG2 samples treated with Sorafenib $10 \mu \mathrm{M}$ and (d) HepG2 samples treated with Sorafenib $40 \mu \mathrm{M}$. Mean fluorescence intensity for FDA and $\mathrm{PI}$ was calculated using Image J. (e) Presto Blue viability assay for treated and untreated HepG2 samples. Scale bar (a-d) $100 \mu \mathrm{m}$. (e) ${ }^{* *} p<0.01,{ }^{* * *} p<0.001,{ }^{* * * *} p<0.0001 . n=4$ per condition. 
In the case of SNU-449, similar to HepG2 samples, Figure 72 showed a decrease in cell survival upon Sorafenib treatment based on the results obtained from Live/Dead staining images (a-d) and Presto Blue assay (e).

Live/Dead image captured for untreated SNU-449 samples (a) showed that almost all cells were alive (90,2\% alive cells). Captured Live/Dead images for SNU-449 samples treated with Sorafenib $2.5 \mu \mathrm{M}$ (b) and Sorafenib $10 \mu \mathrm{M}$ (c) did not show any major changes in cell viability compared to the untreated control samples since almost all the cells were found alive (85.1\% alive cells in b and $83.3 \%$ alive cells in c). Alive SNU449 cells in both Sorafenib $2.5 \mu \mathrm{M}$ (b) and Sorafenib $10 \mu \mathrm{M}$ (c) showed a modification in their morphology compared to alive SNU-449 in untreated samples. Cells showed a larger cytosol/nuclei ration and were characterised by a more accentuated spindle shape. In the case of SNU-449 samples treated with Sorafenib $40 \mu \mathrm{M}$ (d) captured Live/Dead images showed a slightly increased of dead cells compared to all other conditions but even in this condition SNU-449 cells were found more alive than dead (59.8\% alive cells). Compared to HepG2 samples, Live/Dead images showed that SNU-449 were characterised by enhanced chemoresistance to Sorafenib treatment, in particular when the clinical dose of $10 \mu \mathrm{M}$ and toxic dose of $40 \mu \mathrm{M}$ were applied.

Presto Blue assay results (e) showed a Sorafenib dose dependent decrease in cell viability. Compared to untreated control samples, in Sorafenib $2.5 \mu \mathrm{M}$ treated samples SNU-449 were found $82.7 \%$ alive $\left({ }^{* *}, \mathrm{P}<0.01\right)$, in Sorafenib $10 \mu \mathrm{M}$ treated samples cells were found $70.3 \%$ alive ( ${ }^{* * *}, P<0.001$ ), while in Sorafenib $40 \mu \mathrm{M}$ treated samples SNU-449 cells viability decreased up to $63.8 \%\left(^{* * * *}, \mathrm{P}<0.0001\right)$. A statistically significant reduction in SNU-449 cell viability was observed while increasing Sorafenib dose from $2.5 \mu \mathrm{M}$ to $10 \mu \mathrm{M}\left({ }^{* *}, \mathrm{P}<0.01\right)$, but this was not observed while increasing the Sorafenib concentration from $10 \mu \mathrm{M}$ to $40 \mu \mathrm{M}$, indicating a high level of chemoresistance. Similar to Live/Dead results, Presto Blue analysis confirmed that SNU-449 were less sensitive to Sorafenib treatment than HepG2. In the case of Sorafenib clinical dose $10 \mu \mathrm{M} \mathrm{HepG2}$ were found for $58 \%$ alive, while the same dose of chemo drug caused only a 30\% reduction in SNU-449 cells. This suggests that Sorafenib is significantly less effective in SNU-449 cells compared to HepG2 cells.

Compared to the conventional 2D plastic culture system where SNU-449 were found almost 50\% dead upon treatment with Sorafenib $2.5 \mu \mathrm{M}$, the same cells cultured in 
Hep-Gel showed to be much more resistant to the chemo treatment. Indeed, the same Sorafenib dose reduced cell viability up to $83 \%$ and not even the higher Sorafenib dose $(40 \mu \mathrm{M})$ was able to kill $50 \%$ of SNU-449 cells.
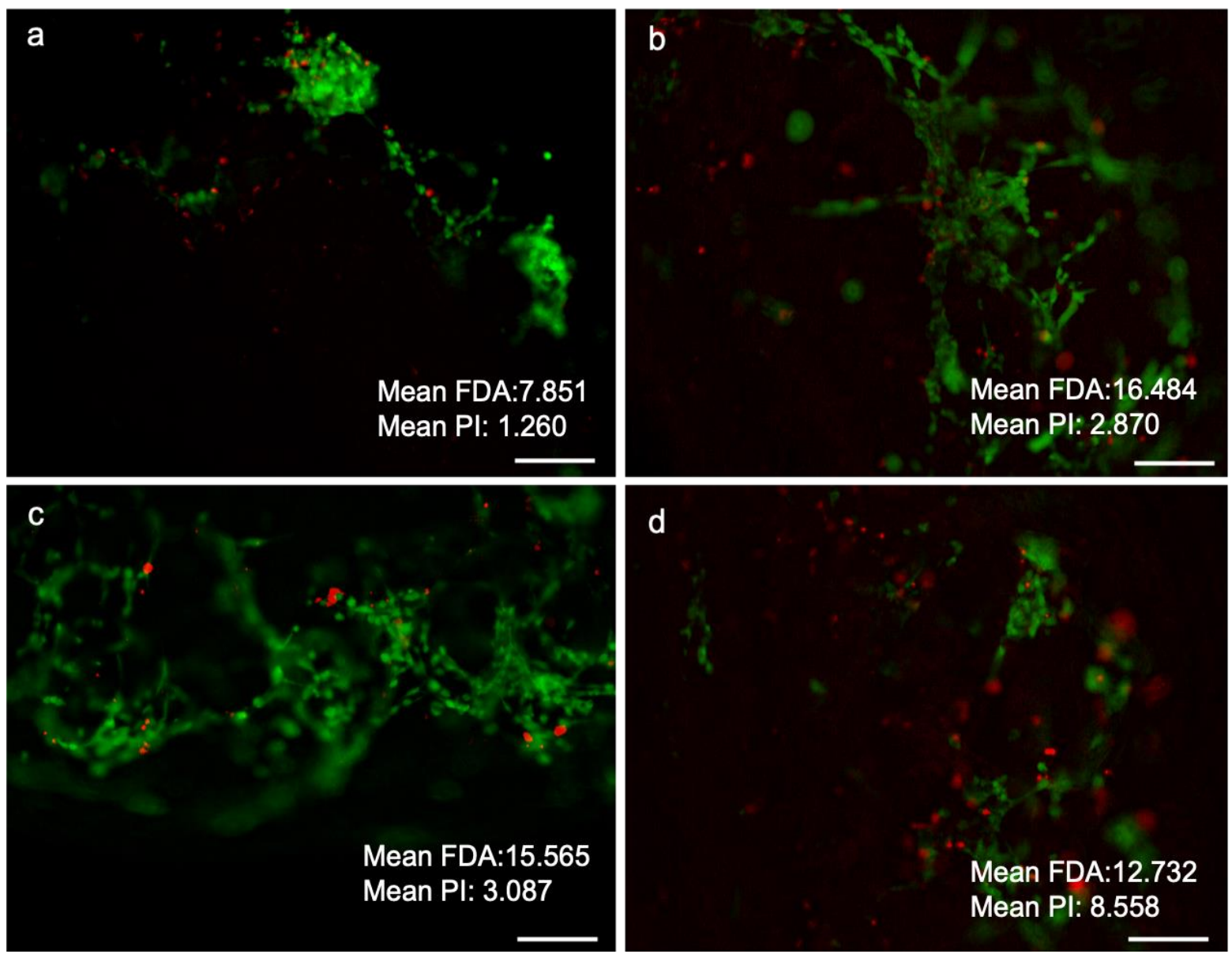

e

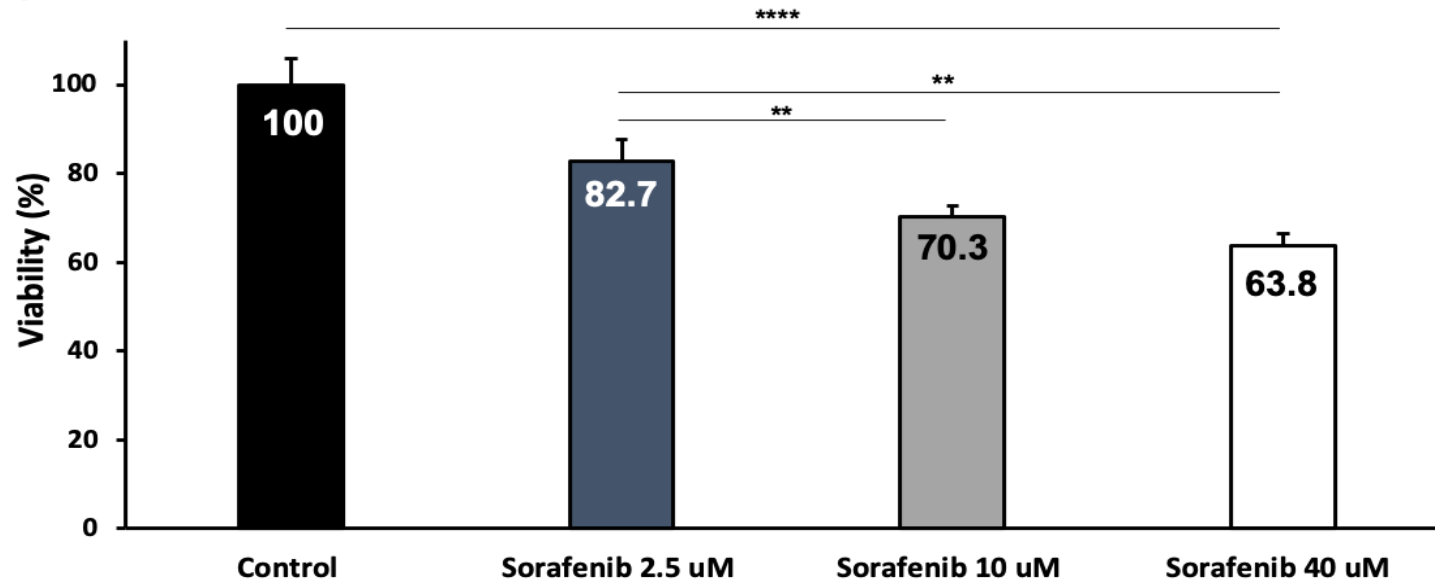

Figure 73. Live/Dead staining images (a-d) of (a) SNU-449 untreated samples, (b) SNU-449 samples treated with Sorafenib $2.5 \mu \mathrm{M}$, (c) SNU-449 samples treated with Sorafenib $10 \mu \mathrm{M}$ and (d) SNU-449 samples treated with Sorafenib $40 \mu \mathrm{M}$. Mean fluorescence intensity was calculated using Image J. (e) Presto Blue viability assay for treated and untreated SNU-449 samples. Scale bar (a-d) $200 \mu \mathrm{m}$. (e) ${ }^{* *} p<0.01,{ }^{* * *} p<0.001,{ }^{* * *} p<0.0001$. $n=4$ per condition. 


\subsubsection{TGF- $\beta 1$ treatment in Hep-Gel 3D culture}

Considering the low level of chemoresistance shown by HepG2 cells upon Sorafenib exposure, especially when cultured in conventional 2D plastic, only SNU-449 were used as HCC cell line to investigate the role of TGF- $\beta 1$ in the epithelial to mesenchymal transition (EMT).

Thus, SNU-449 cells were embedded and cultured in Hep-Gel for 7 days prior to treatment with TGF- $\beta 15 \mathrm{ng} / \mathrm{ml}$ for 48 hours. Live/Dead staining was employed to analyse possible differences in cell viability and proliferation between untreated control samples and TGF- $\beta 1$ treated samples.

Representative Live/Dead images (Figure 73) showed a reduction in alive cells (green signal) and an increase in dead cells (red signal) in TGF- $\beta 1$ treated samples (b) (70.6\% alive cells in b) compared to control samples (a) (90.1\% alive cells in a). This result indicated that TGF- $\beta 1$ affected proliferation and cell viability of SNU-449 cells.
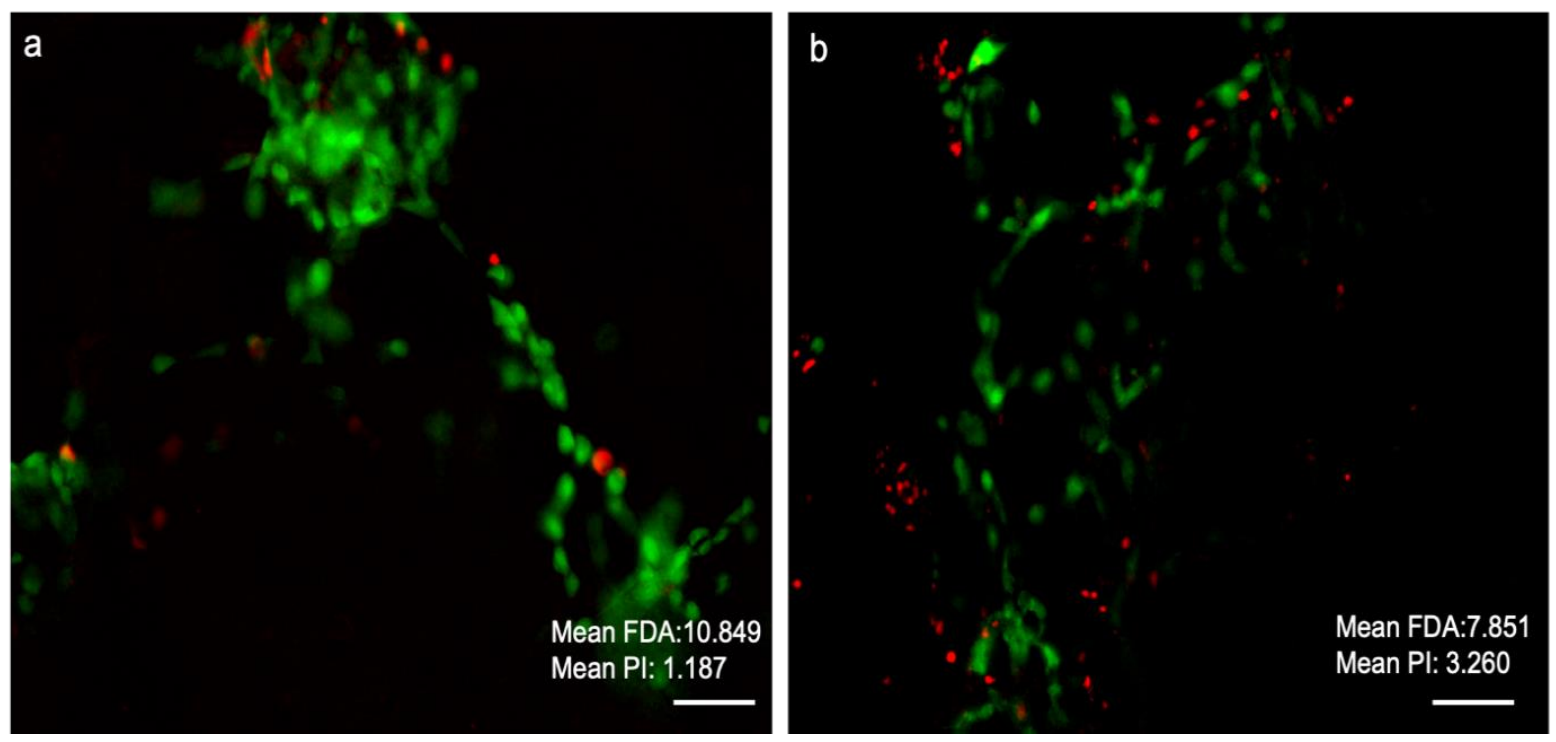

Figure 74. Live/Dead staining images of (a) SNU-449 untreated samples, (b) SNU-449 samples treated with TGF- $\beta 15 \mathrm{ng} / \mathrm{ml}$ for 48 hours. Mean fluorescence intensity for FDA and $\mathrm{PI}$ was calculated using Image J. Scale bar $(\mathrm{a}, \mathrm{b}) 100 \mu \mathrm{m}$.

Quantitative RT-PRC (Figure 74) was carried out to further investigate differences in gene expression induced by TGF- $\beta 1$ treatment. Among the most common genes 
involved in EMT process, E-Cadherin, $\beta$-Catenin, Vimentin and Twist1 were tested using GAPDH as housekeeping gene.

qPCR data showed a significant downregulation in the expression of E-Cadherin (a) $\left({ }^{* * *}, \mathrm{P}<0.0001\right)$ in Hep-Gel treated with TGF- $\beta 1$ compared to untreated control HepGel. On the other hand, $\beta$-Catenin (b) and Vimentin (c) expression were increased after TGF- $\beta 1$ treatment, showing a significant upregulation between untreated control samples and TGF- $\beta 1$ treated samples $\left.{ }^{* * *}, \mathrm{P}<0.001\right)$. These results confirmed the transition from epithelial to mesenchymal phenotype after treatment with TGF- $\beta 1$. Important, Twist1 gene expression (d), showed a significant decrease in expression upon TGF- $\beta 1$ treatment $\left.{ }^{* *}, \mathrm{P}<0.01\right)$. This is a controversial result compared to previous publications $[412,413]$.

a

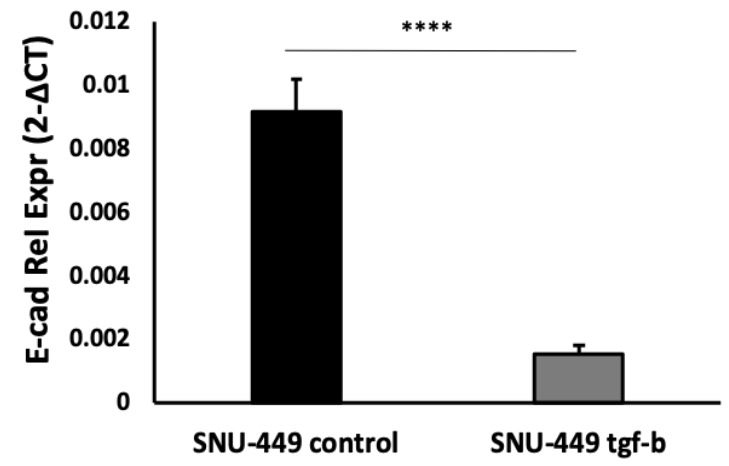

C

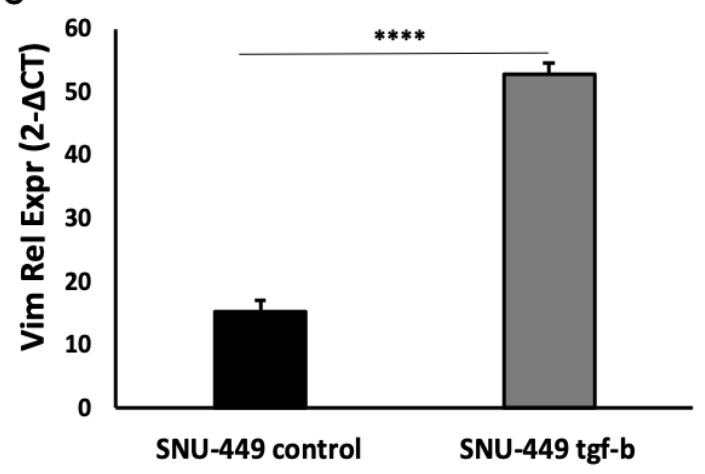

b

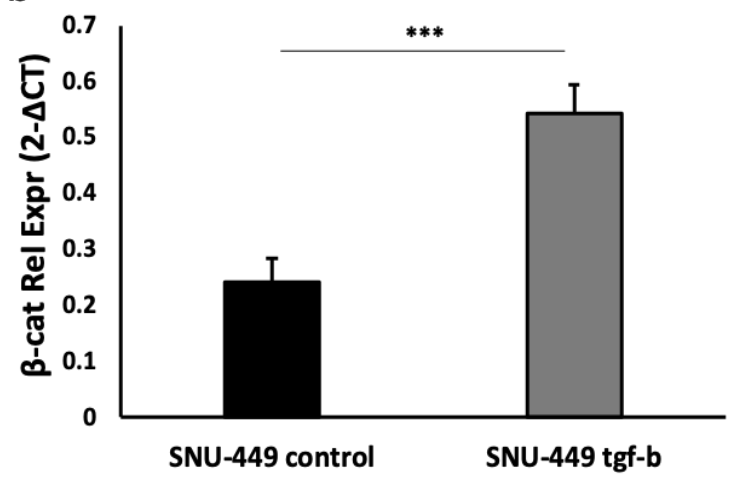

d

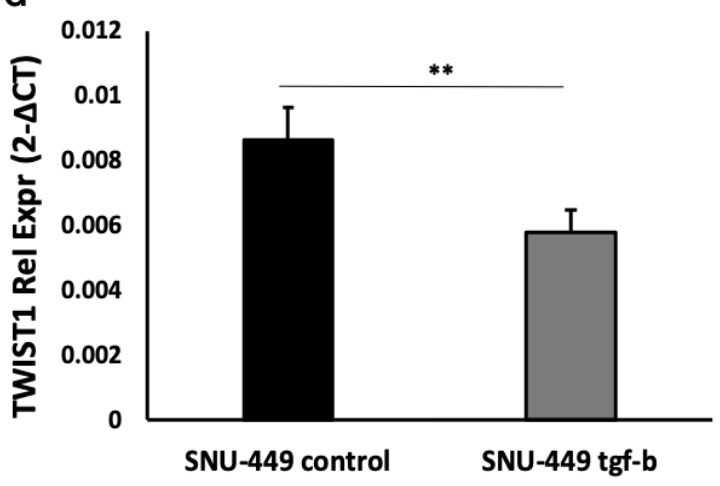

Figure 75. Quantitative analysis of the gene expression of SNU-449 untreated samples and TGF- $\beta 1$ treated treatment. (a) E-Cadherin, (b) $\beta$-Catenin, (c) Vimentin and (d) Twist1 gene level analysis. ${ }^{* *} p<0.01,{ }^{* * *} p<0.001,{ }^{* * * *} p<0.0001 . n=4$ per condition. 


\subsection{Discussion}

\subsubsection{D vs 3D}

Two-dimensional cell monolayers cultured on conventional plastic polystyrene are still the main used platforms for drug screening and development of new therapeutic molecules for the treatment of liver disease [97]. Although advances have been made by using these 2D models, they are not able to reproduce the complexity of human liver. Indeed, cells cultured on conventional plastic polystyrene do not behave in vivo like, since cell-cell interactions are only at a 2D level, cell-ECM interactions are replaced by interaction with plastic, and the stiffness of plastic (approximately $3 \mathrm{gPa}$ ) [414] is dramatically higher compared to a normal liver (2-6KPa) [415], there is no diffusion of oxygen, nutrients and waste products, etc. All these limitations often lead to misleading results during drug screening and development of new therapeutic molecules when performed using 2D plastic models [416].

In this project we evaluated the efficacy to model HCC development in Hep-Gel, an innovative thermoresponsive hydrogel composed of human liver ECM, obtained by the decellularization technique.

Decellularization is an innovative technique that aims to remove all the cellular components from tissues and organs while preserving the mechanical and bioactive properties [408]. For this reason, a model composed of human liver ECM components should provide the essential cues to mimic the liver microenvironment and allow the hepatic cells to behave in vivo like [213].

The Hep-Gel developed in this project presents several advantages compared to other 3D models used to study HCC. First, compared to the 3D models made by synthetic or natural polymers described in the introduction of this Chapter, this new hydrogel is composed of human liver tissue specific ECM, an essential feature to maintain the cells alive, functional and to allow them to behave in vivo like during the in vitro culture. The importance of the ECM components for cell viability and functionality was further confirmed in this Chapter considering the differences observed in HepG2 viability and functionality when cultured in Hep-Gel or Control-Gel. Indeed, HepG2 cultured in Hep- 
Gel were found all alive after both 7 and 13 days of cell culture, while in Control-gel cells were found mainly alive after 7 days but many HepG2 were found dead after 13 days of cell culture. This might be due to a better diffusion of waste, nutrients and oxygen in Hep-gel compared to Control-gel. Moreover, in Hep-Gel the size of the spheroids considerably increased between 7 and 13 days of cell culture, while in Control-gel the size of the spheroids remained almost the same after 7 and 13 days of cell culture, meaning that the gel was not able to fully support the cell growth and the cells started to die when spheroids reached around $100 \mu \mathrm{m}$ diameter.

Secondly, in contrast to the Precision Cut Liver Slices model that can be used over a very limited timeframe, the hepatic cells can be cultured longer in these human liver ECM thermoresponsive hydrogels, allowing researchers to study the tumour development for a longer period and to perform chronic treatments.

Furthermore, Hep-Gel provides some other advantages for example:

1) Cells engraftment and distribution: The preparation of bioengineered Hep-Gel is based on the mix of the Hep-Gel in a liquid state with the cellular component, resulting in a model where cells are evenly distributed throughout the entire Hep-Gel. This is an important advantage of this model compared to those where the cells are seeded on top of the scaffolds or ECM hydrogel (Chapter 2), such as decellularised liver scaffolds [154].

2) Sample preparation: The preparation of each Hep-Gel does not require more than 30 second, and it can be further improved with the use of automated dispensing systems. This is an important advantage of this model for its possible application in the high throughput screening (HTS) of new drugs for the treatment of liver disease.

3) Sample cost-effective: acellular liver scaffolds [154] prepared through decellularization technique are highly time consuming and only one-tenth of samples are prepared from a human liver when compared to Hep-gel. Indeed, an average of 2000 decellularised liver cubes can be prepared from a left lobe of a human liver. On the other hand, from the same left lobe it is possible to obtain an average of 3 grams of ECM powder, resulting in approximately 500 millilitre $(\mathrm{ml})$ of ECM solution and 1 litre (I) of Hep-Gel. Thus, considering that each Hep-Gel is composed of 50 microliters $(\mu \mathrm{l})$, from a liver left lobe it is possible to obtain a total of 20000 hydrogels, thus ten 
times more samples than acellular liver cubes. A similar number of samples can be generated by gelling the human liver ECM solution with Agarose (Chapter 2) or through 3D printing using of ECM-Cellink bio-ink (Chapter 3). Compared to these two ECM based models previously described and discussed in this thesis, Hep-Gel presents several advantages, including a better cell viability, higher time-effective to produce the samples and less total amount of cells needed to perform the experiments. These advantages make the Hep-Gel a better 3D model to be used for high throughput screening (HTS) of new drugs for the treatment of liver disease. Next experiments will be performed using 10-20 microliters $(\mu \mathrm{l})$ of Hep-Gel in 96 well plates in order to further reduce the number of cells needed and increase the number of samples that is possible to obtain from a liver left lobe. This is an essential step toward the high throughput screening (HTS) technology.

\begin{tabular}{|c|c|c|c|}
\hline 3D Platform Features & ECM-Agarose & ECM-bio-ink & Hep-Gel \\
\hline Cell viability & + & + & ++ \\
\hline Cell function & ++ & ++ & ++ \\
\hline Co-culture practicality & -- & ++ & ++ \\
\hline Length in culture & ++ & ++ & ++ \\
\hline Homogeneity & -- & ++ & ++ \\
\hline Toxic cross-linking & ++ & -- & ++ \\
\hline Time-effective & -- & - & ++ \\
\hline $\begin{array}{l}\text { Number of cells } \\
\text { needed }\end{array}$ & -- & - & ++ \\
\hline HTS applicability & -- & - & ++ \\
\hline
\end{tabular}

Table 21. Comparison between the developed ECM-based models described in this thesis. 


\subsubsection{Sorafenib Treatment}

Sorafenib is the first line treatment for advanced HCC patients and for this reason it was selected to evaluate the efficiency of Hep-Gel as a predictive tool to determine the clinical efficacy or chemoresistance of a compound.

In this study, the efficacy of Sorafenib $2.5 \mu$ was compared culturing HepG2 or SNU449 cell lines on conventional 2D plastic cell culture and in Hep-Gel. As expected, the Sorafenib treatment was much more effective when both HepG2 and SNU-449 cell lines were cultured on plastic compared to Hep-Gel. These results are similar to those of several published studies where it was shown that cells in 3D culture are characterised by an increased malignant phenotype and drug resistance compared to standard 2D cultured cells [106, 417-420]. These data confirm that monolayer cultures are poor predictive tools for the clinical efficacy and toxicity of a compound and explain why only $10 \%$ of the anticancer agent successfully complete the clinical trials [98]. Furthermore, most of the compounds fail during phase III of the clinical trials, the most expensive phase of clinical trials [421], thus enormously increasing the cost for drug discovery and development. This highlight the need of in vitro models able to mimic the natural microenvironment of human tissues and organs in order to efficiently discriminate the effective compounds and discard those characterised by lack of efficacy or excessive toxicity.

Clinical trials with Sorafenib showed that this drug can prolong survival in HCC patients, but its efficacy is limited due to development of resistance in cells [422]. The clinical Sorafenib dose $(10 \mu \mathrm{M})$ and a toxic dose $(40 \mu \mathrm{M})$ were applied to HepG2 and SNU-449 cultured in Hep-Gel (after 7 days of culture and treatment for a total of 48 hours) to evaluate the percentage of cell death in the clinical case and the possibility of the HCC cell lines being resistant to Sorafenib. Results showed that both the clinical and toxic doses of Sorafenib caused much less cell death of SNU-449 in comparison to HepG2 cells. In the case of SNU-449, a change in size and morphology upon Sorafenib treatment was observed, and this modification can be linked with chemoresistance. Indeed, several published studies have shown a change in shape and size in chemoresistant cancer cells [423, 424]. Furthermore, the toxic dose of Sorafenib did not cause a statistically significant decrease in cell viability compared to the clinical dose, while HepG2 showed a significant statistical increase in cell death 
compared to the clinical dose. Nevertheless, for both Hepg2 and SNU-449 cell lines there wasn't a substantial difference in terms of cells viability when samples were treated with Sorafenib $10 \mu \mathrm{m}$ or $40 \mu \mathrm{m}$. This is in line with published studies showing that saturation of transporters and targets is responsible for the non-linear dose efficacy [425, 426]. Another possible explanation is the development of chemoresistance to Sorafenib due to the activation of several pathways. This include downstream of Ras/Raf/MEK/ERK signalling pathway, activation of EMT process, over-expression of sorafenib export pumps such as MDR1and BCRP, up-regulation of anti-apoptotic proteins including Bcl-2 and $\mathrm{Mcl}-1$, up-regulation of CYP3A4 and CYP1A1 involved in sorafenib metabolism and over-expression of VEGFR, PDGFR and FGFR [427, 428]. Moreover, a molecule able to kill all the cancer cells in the gels (negative control), such as Eurycomanone or Tamoxifen [429], should have been used to prove that treatments are able to reach also the cells located in the core of the gels

This result, along with the result obtained by Sorafenib treatment when cells were cultured in 2D plastic, confirmed that HepG2 are less aggressive and more responsive to chemotherapy then SNU-449.

These results confirmed that Hep-Gel can be used as a predictive tool to determine the clinical efficacy or chemoresistance of a compound.

\subsubsection{TGF- $\beta 1$ treatment}

Epithelial to Mesenchymal Transition (EMT) is a reversible process in which epithelial cells switch to a mesenchymal phenotype [352]. Transforming Growth Factor $\beta$ (TGF$\beta$ ) is one of the key factors driving the hepatocytes transition from an epithelial phenotype to a mesenchymal phenotype, with consequent loss of cell polarity, dissolution of cell to cell adhesion junctions and increased cell migration, eventually leading to a metastases development [360].

Considering the hepatoblastoma behaviour of HepG2 observed upon Sorafenib treatment, the effect of TGF- $\beta 15 \mathrm{ng} / \mathrm{ml}$ (after 7 days of culture and for a total of 48 hours) was investigated only using SNU-449 cells cultured in Hep-Gel. 
Live/dead analysis of the samples after TGF- $\beta 1$ treatment showed a lower number of alive cells and an increased number of dead cells in treated samples compared to untreated control samples. This result is consistent with other published studies where it was shown that TGF- $\beta 1$ reduced SNU-449 cell growth and increased the percentage of apoptotic cells compared to untreated samples [430]. Gene expression analysis was performed to investigate the effect of TGF- $\beta 1$ on Vimentin, E-Cadherin, $\beta$-Catenin and Twist1 gene levels. Expression of the epithelial marker E-Cadherin was significantly down-regulated after 48 hours of TGF- $\beta 1$ treatment, suggesting advances in the EMT stage of the SNU-449 cell line towards a more mesenchymal phenotype. At the same time the investigated mesenchymal markers Vimentin and $\beta$-Catenin were clearly up-regulated, further confirming such transition toward a mesenchymal phenotype. The expression of Twist 1 is correlated with advancement in EMT process, invasion and metastasis development [431]. The performed gene expression analysis showed a significant decrease between the control and the treated samples, which disagrees with previous studies [412, 413, 431]. This result indicates that beside Twist 1 there are other transcription factors that play a key role in the downregulation of E-Cadherin and consequent upregulation of Vimentin during the epithelial to mesenchymal transition. A deeper investigation of possible other transcription factors involved in this process should be carried out in order to identify and validate new cancer targets.

In conclusion, the results described in this Chapter 4 confirmed that Hep-Gel could be used as predictive model to evaluate the anti-cancer effect of new compounds as well as to study the cellular mechanisms underlying the EMT processes. 


\section{Chapter 5: General discussion and future work}

The work presented in this thesis describes for the first time the development of tissue specific ECM hydrogels starting from human livers unsuitable for transplantation. The aim of this work was to develop a more physiological in vitro 3D model that could replace the conventional $2 \mathrm{D}$ plastic cell cultures for the high throughput screening (HTS) of new drugs and target validation for the treatment of liver diseases such as liver fibrosis and hepatocellular carcinoma (HCC) and for assessing drugs-related hepatotoxicity. A more efficient in vitro drug screening and development can also reduce the use of laboratory animals, decreasing the time needed and the costs of drug discovery and development process. The other ambitious aim was to use the developed human liver ECM hydrogels for in vivo cell therapy in order to improve survival and engraftment of transplanted cells and therefore enhance the successful rate of this clinical approach for the treatment of metabolic liver disorders.

Previous studies had described the development of ECM-based hydrogels through the decellularization of different organs, including porcine small intestinal submucosa [184], porcine liver [186], porcine urinary bladder [113] and porcine adipose tissue [187]. However, the physical and biochemical composition of the ECM is tissuespecific. Porcine liver from the physiological and anatomical point of view is considered to be the most similar to human liver, but it is characterised by well-defined lobules delineated by connective tissue, which are not present in healthy human livers but actually more typical of fibrotic human livers [213]. Therefore, although porcine liver ECM is considered to be the closest to human liver ECM, these changes in the ECM composition might alter cell signalling leading to a non in vivo like behaviour of hepatic cells [191]. In conclusion, to recreate the physiological microenvironment for the in vitro culture of the human hepatic cells one would favour human liver as most suitable source of ECM.

So far, all published protocols used for the development of ECM-based hydrogels employ pepsin for the digestion of the ECM powder. This xenogeneic enzyme cannot be removed from the ECM solution, therefore ECM-hydrogels prepared with above mentioned digestion step contain a high quantity of this enzyme and thus present a 
disadvantage for the in vivo cell transplantation. Thus, the main advantage of the new developed protocol, and an important step for future applications, is that the obtained human liver ECM solution does not contain xenogeneic enzymes. Furthermore, one important difference that was noticed is that the human liver ECM solution did not show self-assembling properties previously demonstrated by others xenogeneic organs ECM solutions, such as porcine liver ECM solution, porcine small intestine mucosa ECM solution and porcine urinary bladder ECM solution further indicating peculiar species specific characteristics.

To overcome this problem, as described in Chapter 2, gelling agents such as agarose and agar were first employed to gel the human liver ECM solution. These two gelling agents were selected because they are biocompatible, non-cancerogenic, non-toxic, biodegradable and already used for clinical applications as dermal filler [214]. ECM hydrogels containing $0.25 \%$ of agarose showed a stiffness comparable to that of human healthy livers, essential to preserve the in vivo cell phenotype and activity, adequate porosity, important to allow the diffusion of oxygen and nutrients, and bioactive cues as confirmed by the positive histological and immunohistochemical staining for the most abundant ECM proteins. Comparable results in terms of in vitro biocompatibility were observed between ECM-Agarose hydrogels and acellular liver cubes, the other 3D model developed in our lab [154]. Although in ECM-hydrogels the native ultrastructure of healthy livers is not preserved as in the acellular liver cubes, the main advantage of this novel 3D platform is the possibility of large scale production of gels within a reasonable time, thus more suitable for HTS purposes. ECM hydrogels were able to support the differentiation of Induced Pluripotent Stem Cells (IPSc) towards hepatocytes-like cells, important to overcome the limitation in available hepatocytes for in vitro and in vivo applications, and were also able to promote HepG2 survival and engraftment when transplanted into the omentum of immune deficient mice, without causing important systemic and local inflammation/fibrogenesis. These are encouraging results to overcome the current cell therapy limitations.

The major limitations of the new developed ECM-Agarose hydrogel system that could compromise their HTS applicability include: (i) the experimental variability due to different cell attachment into the hydrogels, (ii) co-culture limitations due to the impossibility to predict the cell attachment and (iii) the high number of cells needed. 
As described in Chapter 3, to overcome the limitations of ECM-agarose hydrogels, the human liver ECM solution was used as a bio-ink for 3D printing in combination with nanocellulose (Cellink bio-ink), a supporting material for bio-printing. Mixing the cells before bio-printing allowed to reduce the number of cells needed and automatically increased the consistency between samples/condition thus reducing experimental variability. Another advantage of this process is the possibility to increase the complexity of the sample by co-culturing hepatic cells while still maintaining the correct in-vivo like cell distribution. Compared to other ECM-based bio-inks described in literature, the use of human liver ECM in combination with Cellink bio-ink allowed to prepare human tissue-specific constructs in order to better mimic the human liver microenvironment, and bio-print under physiological conditions, room temperature and very low extrusion pressure in order to reduce the mechanical stress to the cells. These advantages were confirmed by the promising results obtained by bio-printing hepatic cell lines (LX2 and HepG2) and human primary hepatocytes. Unfortunately, 3D bio-printing presented also several problems including: (i) the use of calcium chloride at toxic concentration as cross-linking reagent, (ii) the numerous mixing steps required to obtain a homogeneous bio-ink that cause a loss of biomaterial during the process and hamper the sterility of the same, and (iii) the several washes required after bio-printing to remove the cross-linking agent which considerably increased the time needed to prepare the samples. All these technical limitations and the still too high number of cells required compromise the applicability of ECM bio-ink for high throughput screening of new drugs.

As described in Chapter 4, the technical limitations and high number of cells needed for bio-printing process were overcome through the development of Hep-Gel, an innovative ECM-based hydrogel composed of human liver ECM and a synthetic thermoresponsive co-polymer. The resulting Hep-Gel is a thermo-responsive hydrogel similar to Matrigel but with human tissue specific ECM. The efficiency of Hep-Gel bioengineered HepG2 or SNU-449 cells as in vitro HCC model was validated through TGF- $\beta 1$ and Sorafenib treatment. Important, results described in Chapter 4 showed that Hep-Gel is a better predictive model compared to 2D plastic cultures to evaluate the drug anti-cancer effect. 
As soon as possible, Hep-Gel will be fully characterised in terms of ECM proteins composition through proteomic, mechanical properties through rheology, and of microarchitecture through SEM.

Moreover, several steps are still required to develop an in vitro model able to better mimic the diseased microenvironment of HCC. This include (i) co-culture of different types of hepatic cells to mimic the heterogeneity of this disease, (ii) perform the experiment in a reduced oxygen condition to mimic the in vivo tumour hypoxia, and (iii) develop cirrhotic ECM solution to better mimic the HCC microenvironment considering that $90 \%$ of $\mathrm{HCC}$ cases is developed in fibrotic or cirrhotic subjects [309].

Furthermore, other essential steps toward the high throughput screening (HTS) technology are necessary. Moving in this direction, an automated system will be employed to dispense 10-20 microliters of bioengineered Hep-Gel with hepatic cells in 96 well plates.

It is in my future research development plan to investigate the efficacy of Hep-Gel for in vivo cell transplantation. Bioengineered Hep-Gel with primary human hepatocytes or IPSc differentiated towards hepatocytes-like cells will be implanted in mice and cell survival, engraftment and functionality will be evaluated. 


\section{References}

1. Abdel-Misih, S.R. and M. Bloomston, Liver anatomy. Surg Clin North Am, 2010. 90(4): p. 643-53.

2. Irwin M. Arias, e.a., The Liver Biology and Pathobiology. 5th Edition Vol. 5th Edition 2009, Wiley.

3. Xu, S., et al., Quantification of liver fibrosis via second harmonic imaging of the Glisson's capsule from liver surface. J Biophotonics, 2016. 9(4): p. 351-63.

4. Bismuth, H.J.W.J.o.S., Surgical anatomy and anatomical surgery of the liver. 1982. 6(1): p. 3-9.

5. Z., K., Cooperation of Liver Cells in Health and Disease. Advances in Anatomy Embryology and Cell Biology. Vol. vol 161. 2001, Springer, Berlin, Heidelberg.

6. Godoy, P., et al., Recent advances in 2D and 3D in vitro systems using primary hepatocytes, alternative hepatocyte sources and non-parenchymal liver cells and their use in investigating mechanisms of hepatotoxicity, cell signaling and ADME. Arch Toxicol, 2013. 87(8): p. 1315-530.

7. Martinez-Hernandez, A. and P.S. Amenta, The hepatic extracellular matrix. I. Components and distribution in normal liver. Virchows Arch A Pathol Anat Histopathol, 1993. 423(1): p. 1-11.

8. Bilzer, M., F. Roggel, and A.L. Gerbes, Role of Kupffer cells in host defense and liver disease. Liver Int, 2006. 26(10): p. 1175-86.

9. Coulouarn, C., et al., Hepatocyte-stellate cell cross-talk in the liver engenders a permissive inflammatory microenvironment that drives progression in hepatocellular carcinoma. Cancer Res, 2012. 72(10): p. 2533-42.

10. Bataller, R. and D.A. Brenner, Liver fibrosis. J Clin Invest, 2005. 115(2): p. 20918.

11. Rombouts, K., Chapter 2 - Hepatic Stellate Cell Culture Models, in Stellate Cells in Health and Disease, C.R. Gandhi and M. Pinzani, Editors. 2015, Academic Press: Boston. p. 15-27.

12. Hautekeete, M.L. and A. Geerts, The hepatic stellate (Ito) cell: its role in human liver disease. Virchows Arch, 1997. 430(3): p. 195-207.

13. Friedman, S.L., Molecular regulation of hepatic fibrosis, an integrated cellular response to tissue injury. The Journal of biological chemistry, 2000. 275(4): p. 2247-2250.

14. University, C. http://eclinpath.com/chemistry/liver/liver-structure-andfunction/liverlobule/. 27/08/2019]. 
15. Wells, R.G., The role of matrix stiffness in regulating cell behavior. Hepatology, 2008. 47(4): p. 1394-400.

16. Hynes, R.O., Extracellular matrix: not just pretty fibrils. Science (New York, N.Y.), 2009. 326(5957): p. 1216-1219.

17. Flaim, C.J., S. Chien, and S.N. Bhatia, An extracellular matrix microarray for probing cellular differentiation. Nat Methods, 2005. 2(2): p. 119-25.

18. Schuppan, D., Structure of the extracellular matrix in normal and fibrotic liver: collagens and glycoproteins. Semin Liver Dis, 1990. 10(1): p. 1-10.

19. Rojkind, M., M.A. Giambrone, and L. Biempica, Collagen types in normal and cirrhotic liver. Gastroenterology, 1979. 76(4): p. 710-9.

20. Bernal, W. and J. Wendon, Acute Liver Failure. New England Journal of Medicine, 2013. 369(26): p. 2525-2534.

21. Figorilli, F., et al., Development of an organ failure score in acute liver failure for transplant selection and identification of patients at high risk of futility. PLOS ONE, 2017. 12(12): p. e0188151.

22. Bernal, W., et al., Lessons from look-back in acute liver failure? A single centre experience of 3300 patients. J Hepatol, 2013. 59(1): p. 74-80.

23. Nelson, N.P. and T.V. Murphy, Hepatitis A: The Changing Epidemiology of Hepatitis A. Clinical liver disease, 2013. 2(6): p. 227-230.

24. Hoofnagle, J.H., K.E. Nelson, and R.H. Purcell, Hepatitis E. N Engl J Med, 2012. 367(13): p. 1237-44.

25. Khuroo, M.S. and S. Kamili, Aetiology and prognostic factors in acute liver failure in India. J Viral Hepat, 2003. 10(3): p. 224-31.

26. Reuben, A., D.G. Koch, and W.M. Lee, Drug-induced acute liver failure: results of a U.S. multicenter, prospective study. Hepatology, 2010. 52(6): p. 2065-76.

27. Tanne, J., Paracetamol causes most liver failure in UK and US. BMJ : British Medical Journal, 2006. 332(7542): p. 628-628.

28. Statistics, O.f.N. Number of drug-related deaths where paracetamol was mentioned on the death certificate, England and Wales, deaths registered in 1993 to $2015.2016 ;$ Available from: https://www.ons.gov.uk/peoplepopulationandcommunity/birthsdeathsandmarri ages/deaths/adhocs/006190numberofdrugrelateddeathsmentioningparacetam olregionsofenglandandwales 19932015 registrations.

29. Hansen, K. and S. Horslen, Metabolic liver disease in children. Liver Transpl, 2008. 14(4): p. 391-411.

30. Perlmutter, D.H., Metabolic Liver Disease. 2002. 35: p. S24-S28. 
31. de Serres, F.J., Alpha-1 antitrypsin deficiency is not a rare disease but a disease that is rarely diagnosed. Environ Health Perspect, 2003. 111(16): p. 1851-4.

32. Teckman, J.H., Liver disease in alpha-1 antitrypsin deficiency: current understanding and future therapy. Copd, 2013. 10 Suppl 1: p. 35-43.

33. Clark, V.C., Liver Transplantation in Alpha-1 Antitrypsin Deficiency. Clin Liver Dis, 2017. 21(2): p. 355-365.

34. Ala, A., et al., Wilson's disease. Lancet, 2007. 369(9559): p. 397-408.

35. M., Y., Urea Cycle in Basic Neurochemistry: Molecular, Cellular and Medical Aspects, A.B. Siegel GJ, Albers RW, et al., editors, Editor. 1999: Philadelphia: Lippincott-Raven.

36. Leonard, J.V. and A.A. Morris, Urea cycle disorders. Semin Neonatol, 2002. 7(1): p. 27-35.

37. Tuchman, M., The clinical, biochemical, and molecular spectrum of ornithine transcarbamylase deficiency. J Lab Clin Med, 1992. 120(6): p. 836-50.

38. Jansen, P.L., Diagnosis and management of Crigler-Najjar syndrome. Eur J Pediatr, 1999. 158 Suppl 2: p. S89-94.

39. Schauer, R., et al., Treatment of Crigler-Najjar type 1 disease: relevance of early liver transplantation. J Pediatr Surg, 2003. 38(8): p. 1227-31.

40. Lindblad, B., S. Lindstedt, and G. Steen, On the enzymic defects in hereditary tyrosinemia. Proc Natl Acad Sci U S A, 1977. 74(10): p. 4641-5.

41. Endo, F. and M.-S. Sun, Tyrosinaemia type I and apoptosis of hepatocytes and renal tubular cells. Journal of Inherited Metabolic Disease, 2002. 25(3): p. 227234.

42. Mieles, L.A., et al., Liver Transplantation for Tyrosinemia: A Review of 10 Cases from the University of Pittsburgh. Digestive diseases and sciences, 1990. 35(1): p. 153-157.

43. Leumann, E. and B. Hoppe, The primary hyperoxalurias. J Am Soc Nephrol, 2001. 12(9): p. 1986-93.

44. Hoppe, B. and C.B. Langman, A United States survey on diagnosis, treatment, and outcome of primary hyperoxaluria. Pediatr Nephrol, 2003. 18(10): p. 98691.

45. Özen, H., Glycogen storage diseases: New perspectives. World Journal of Gastroenterology : WJG, 2007. 13(18): p. 2541-2553.

46. Bove, K.E., et al., Bile acid synthetic defects and liver disease: a comprehensive review. Pediatr Dev Pathol, 2004. 7(4): p. 315-34. 
47. Lemonde, H.A., et al., Mutations in SRD5B1 (AKR1D1), the gene encoding delta(4)-3-oxosteroid 5beta-reductase, in hepatitis and liver failure in infancy. Gut, 2003. 52(10): p. 1494-9.

48. Gilbert, F., Postscript: a status report on hemochromatosis population screening. Genet Test, 2000. 4(2): p. 229-31.

49. Stickel, F., et al., Evaluation of genome-wide loci of iron metabolism in hereditary hemochromatosis identifies PCSK7 as a host risk factor of liver cirrhosis. Hum Mol Genet, 2014. 23(14): p. 3883-90.

50. Kowdley, K.V., et al., Survival after liver transplantation in patients with hepatic iron overload: the national hemochromatosis transplant registry. Gastroenterology, 2005. 129(2): p. 494-503.

51. Emanuele, D., I. Tuason, and Q.T. Edwards, HFE-associated hereditary hemochromatosis: overview of genetics and clinical implications for nurse practitioners in primary care settings. J Am Assoc Nurse Pract, 2014. 26(3): p. 113-22.

52. S. K. Sarin, R.M. Global Burden Of Liver Disease: A True Burden on Health Sciences and Economies!!

53. Li, W.X., et al., Histological differentiation predicts post-liver transplantation survival time. Clin Res Hepatol Gastroenterol, 2014. 38(2): p. 201-8.

54. Hansen, L., Y. Yan, and S.J. Rosenkranz, The power of the liver transplant waiting list: a case presentation. American journal of critical care : an official publication, American Association of Critical-Care Nurses, 2014. 23(6): p. 510515.

55. Mazza, G., et al., Hepatic regenerative medicine. J Hepatol, 2015. 63(2): p. 523-4.

56. Allen, J.W., T. Hassanein, and S.N. Bhatia, Advances in bioartificial liver devices. Hepatology, 2001. 34(3): p. 447-55.

57. Yu, Y., et al., Cell therapies for liver diseases. Liver Transpl, 2012. 18(1): p. 921.

58. Sokal, E.M., et al., Hepatocyte transplantation in a 4-year-old girl with peroxisomal biogenesis disease: technique, safety, and metabolic follow-up. Transplantation, 2003. 76(4): p. 735-8.

59. Nyberg, S.L., Bridging the gap: advances in artificial liver support. Liver Transpl, 2012. 18 Suppl 2: p. S10-4.

60. Tan, H.K., Molecular adsorbent recirculating system (MARS). Ann Acad Med Singapore, 2004. 33(3): p. 329-35.

61. Joseph, N. and L. Kumar, Liver support devices: Bridge to transplant or recovery. 2017. 6(2): p. 807-812. 
62. Selden, C., et al., Evaluation of encapsulated liver cell spheroids in a fluidisedbed bioartificial liver for treatment of ischaemic acute liver failure in pigs in a translational setting. PLoS One, 2013. 8(12): p. e82312.

63. Khalil, M., et al., Human hepatocyte cell lines proliferating as cohesive spheroid colonies in alginate markedly upregulate both synthetic and detoxificatory liver function. J Hepatol, 2001. 34(1): p. 68-77.

64. Selden, C., et al., A clinical-scale BioArtificial Liver, developed for GMP, improved clinical parameters of liver function in porcine liver failure. Scientific Reports, 2017. 7(1): p. 14518.

65. Mazza, G., et al., Liver tissue engineering: From implantable tissue to whole organ engineering. Hepatol Commun, 2018. 2(2): p. 131-141.

66. Dhawan, A., et al., Human hepatocyte transplantation: current experience and future challenges. Nat Rev Gastroenterol Hepatol, 2010. 7(5): p. 288-98.

67. Dorko, K., et al., A new technique for isolating and culturing human hepatocytes from whole or split livers not used for transplantation. Cell Transplant, 1994. 3(5): p. 387-95.

68. Mitry, R.R., R.D. Hughes, and A. Dhawan, Progress in human hepatocytes: isolation, culture \& cryopreservation. Semin Cell Dev Biol, 2002. 13(6): p. 4637.

69. Lee, S.W., et al., Hepatocyte transplantation: state of the art and strategies for overcoming existing hurdles. Ann Hepatol, 2004. 3(2): p. 48-53.

70. Jain, E., A. Damania, and A. Kumar, Biomaterials for liver tissue engineering. Hepatol Int, 2014. 8(2): p. 185-97.

71. O'Brien, F.J., Biomaterials \& scaffolds for tissue engineering. Materials Today, 2011. 14(3): p. 88-95.

72. Orive, G., et al., Cell encapsulation: promise and progress. Nat Med, 2003. 9(1): p. 104-7.

73. Orive, G., et al., Cell encapsulation: technical and clinical advances. Trends Pharmacol Sci, 2015. 36(8): p. 537-46.

74. Jitraruch, S., et al., Alginate microencapsulated hepatocytes optimised for transplantation in acute liver failure. PLoS One, 2014. 9(12): p. e113609.

75. Kim, A.R., et al., Reduction of inflammatory reaction in the use of purified alginate microcapsules. J Biomater Sci Polym Ed, 2013. 24(9): p. 1084-98.

76. Cao, N., X.B. Chen, and D.J. Schreyer, Influence of Calcium Ions on Cell Survival and Proliferation in the Context of an Alginate Hydrogel. ISRN Chemical Engineering, 2012. 2012: p. 9. 
77. Murphy, S.V. and A. Atala, 3D bioprinting of tissues and organs. Nat Biotechnol, 2014. 32(8): p. 773-85.

78. Hospodiuk, M., et al., The bioink: A comprehensive review on bioprintable materials. Biotechnol Adv, 2017. 35(2): p. 217-239.

79. Kim, B.S., et al., Decellularized extracellular matrix: a step towards the next generation source for bioink manufacturing. Biofabrication, 2017. 9(3): p. 034104.

80. Lee, H., et al., Development of Liver Decellularized Extracellular Matrix Bioink for Three-Dimensional Cell Printing-Based Liver Tissue Engineering. Biomacromolecules, 2017. 18(4): p. 1229-1237.

81. Aravamudhan, A., et al., Chapter 4 - Natural Polymers: Polysaccharides and Their Derivatives for Biomedical Applications, in Natural and Synthetic Biomedical Polymers, S.G. Kumbar, C.T. Laurencin, and M. Deng, Editors. 2014, Elsevier: Oxford. p. 67-89.

82. Nguyen, M.K. and D.S. Lee, Injectable biodegradable hydrogels. Macromol Biosci, 2010. 10(6): p. 563-79.

83. Hussey, G.S., J.L. Dziki, and S.F. Badylak, Extracellular matrix-based materials for regenerative medicine. Nature Reviews Materials, 2018. 3(7): p. 159-173.

84. Sharma, N.S., D. Nagrath, and M.L. Yarmush, Adipocyte-derived basement membrane extract with biological activity: applications in hepatocyte functional augmentation in vitro. Faseb j, 2010. 24(7): p. 2364-74.

85. Fu, R.-H., et al., Decellularization and Recellularization Technologies in Tissue Engineering. Cell Transplantation, 2014. 23(4-5): p. 621-630.

86. Keane, T.J., I.T. Swinehart, and S.F. Badylak, Methods of tissue decellularization used for preparation of biologic scaffolds and in vivo relevance. Methods, 2015. 84: p. 25-34.

87. Gilpin, A. and Y. Yang, Decellularization Strategies for Regenerative Medicine: From Processing Techniques to Applications. Biomed Res Int, 2017. 2017: p. 9831534.

88. Lee, J.S., et al., Liver extracellular matrix providing dual functions of twodimensional substrate coating and three-dimensional injectable hydrogel platform for liver tissue engineering. Biomacromolecules, 2014. 15(1): p. 20618.

89. Zhou, P., et al., Decellularized liver matrix as a carrier for the transplantation of human fetal and primary hepatocytes in mice. Liver Transpl, 2011. 17(4): p. $418-27$.

90. Guglielmi, A., et al., How much remnant is enough in liver resection? Dig Surg, 2012. 29(1): p. 6-17. 
91. Uygun, B.E. and M.L. Yarmush, Engineered liver for transplantation. Curr Opin Biotechnol, 2013. 24(5): p. 893-9.

92. Sussman, N.L. and J.H. Kelly, Artificial liver: a forthcoming attraction. Hepatology, 1993. 17(6): p. 1163-4.

93. Edmondson, R., et al., Three-dimensional cell culture systems and their applications in drug discovery and cell-based biosensors. Assay Drug Dev Technol, 2014. 12(4): p. 207-18.

94. Kola, I., The state of innovation in drug development. Clin Pharmacol Ther, 2008. 83(2): p. 227-30.

95. Hwang, T.J., et al., Failure of Investigational Drugs in Late-Stage Clinical Development and Publication of Trial Results. JAMA Intern Med, 2016. 176(12): p. 1826-1833.

96. Fogel, D.B., Factors associated with clinical trials that fail and opportunities for improving the likelihood of success: A review. Contemporary clinical trials communications, 2018. 11: p. 156-164.

97. Kunz-Schughart, L.A., et al., The use of 3-D cultures for high-throughput screening: the multicellular spheroid model. J Biomol Screen, 2004. 9(4): p. 273-85.

98. Breslin, S. and L. O'Driscoll, Three-dimensional cell culture: the missing link in drug discovery. Drug Discov Today, 2013. 18(5-6): p. 240-9.

99. Schuetz, E.G., et al., Regulation of gene expression in adult rat hepatocytes cultured on a basement membrane matrix. J Cell Physiol, 1988. 134(3): p. 30923.

100. Dunn, J.C., R.G. Tompkins, and M.L. Yarmush, Long-term in vitro function of adult hepatocytes in a collagen sandwich configuration. Biotechnol Prog, 1991. 7(3): p. 237-45.

101. Tchaparian, E.H., et al., Effect of culture time on the basal expression levels of drug transporters in sandwich-cultured primary rat hepatocytes. Drug Metab Dispos, 2011. 39(12): p. 2387-94.

102. Kotani, N., et al., Culture period-dependent changes in the uptake of transporter substrates in sandwich-cultured rat and human hepatocytes. Drug Metab Dispos, 2011. 39(9): p. 1503-10.

103. Birgersdotter, A., R. Sandberg, and I. Ernberg, Gene expression perturbation in vitro--a growing case for three-dimensional (3D) culture systems. Semin Cancer Biol, 2005. 15(5): p. 405-12.

104. Bhadriraju, K. and C.S. Chen, Engineering cellular microenvironments to improve cell-based drug testing. Drug Discov Today, 2002. 7(11): p. 612-20. 
105. Nussler, A.K., et al., The suitability of hepatocyte culture models to study various aspects of drug metabolism. Altex, 2001. 18(2): p. 91-101.

106. Horning, J.L., et al., 3-D tumor model for in vitro evaluation of anticancer drugs. Mol Pharm, 2008. 5(5): p. 849-62.

107. Stokes, W.S., Best practices for the use of animals in toxicological research and testing. Ann N Y Acad Sci, 2011. 1245: p. 17-20.

108. Morgan, E., Regulation of Hepatic Drug Metabolizing Enzymes in Animal Models of Infectious and Inflammatory Diseases. 2006.

109. Abass, K., et al., Comparative metabolism of benfuracarb in in vitro mammalian hepatic microsomes model and its implications for chemical risk assessment. Toxicol Lett, 2014. 224(2): p. 290-9.

110. Smith, D.A., Species differences in metabolism and pharmacokinetics: are we close to an understanding? Drug Metab Rev, 1991. 23(3-4): p. 355-73.

111. Freires, I.A., et al., Alternative Animal and Non-Animal Models for Drug Discovery and Development: Bonus or Burden? Pharm Res, 2017. 34(4): p. 681-686.

112. Marga, F., et al., Developmental biology and tissue engineering. Birth Defects Res C Embryo Today, 2007. 81(4): p. 320-8.

113. Freytes, D.O., et al., Preparation and rheological characterization of a gel form of the porcine urinary bladder matrix. Biomaterials, 2008. 29(11): p. 1630-7.

114. Zietarska, M., et al., Molecular description of a $3 D$ in vitro model for the study of epithelial ovarian cancer (EOC). Mol Carcinog, 2007. 46(10): p. 872-85.

115. O'Brien, L.E., M.M. Zegers, and K.E. Mostov, Opinion: Building epithelial architecture: insights from three-dimensional culture models. Nat Rev Mol Cell Biol, 2002. 3(7): p. 531-7.

116. Gumbiner, B.M., Regulation of cadherin-mediated adhesion in morphogenesis. Nat Rev Mol Cell Biol, 2005. 6(8): p. 622-34.

117. Lee, S.A., et al., Spheroid-based three-dimensional liver-on-a-chip to investigate hepatocyte-hepatic stellate cell interactions and flow effects. Lab Chip, 2013. 13(18): p. 3529-37.

118. Ding, C., et al., A Cell-type-resolved Liver Proteome. Mol Cell Proteomics, 2016. 15(10): p. 3190-3202.

119. Thomas, R.J., et al., The effect of three-dimensional co-culture of hepatocytes and hepatic stellate cells on key hepatocyte functions in vitro. Cells Tissues Organs, 2005. 181(2): p. 67-79. 
120. Pan, K., et al., [Evaluation of co-cultured CL-1 hepatocytes and hepatic stellate cells in rotatory cell culture system]. Nan Fang Yi Ke Da Xue Xue Bao, 2013. 33(6): p. 902-5.

121. Cukierman, E., et al., Taking cell-matrix adhesions to the third dimension. Science, 2001. 294(5547): p. 1708-12.

122. Hynes, R.O., Integrins: bidirectional, allosteric signaling machines. Cell, 2002. 110(6): p. 673-87.

123. Legate, K.R., S.A. Wickstrom, and R. Fassler, Genetic and cell biological analysis of integrin outside-in signaling. Genes Dev, 2009. 23(4): p. 397-418.

124. Geiger, B., et al., Transmembrane Crosstalk Between the Extracellular MatrixCytoskeleton Crosstalk. Nature Reviews Molecular Cell Biology, 2001. 2: p. 793-805.

125. Mazza, G., W. Al-Akkad, and K. Rombouts, Engineering in vitro models of hepatofibrogenesis. Adv Drug Deliv Rev, 2017. 121: p. 147-157.

126. Kim, K., et al., Preserved liver-specific functions of hepatocytes in 3D co-culture with endothelial cell sheets. Biomaterials, 2012. 33(5): p. 1406-13.

127. Landry, J., et al., Spheroidal aggregate culture of rat liver cells: histotypic reorganization, biomatrix deposition, and maintenance of functional activities. $J$ Cell Biol, 1985. 101(3): p. 914-23.

128. Fennema, E., et al., Spheroid culture as a tool for creating $3 D$ complex tissues. Trends Biotechnol, 2013. 31(2): p. 108-15.

129. Tostoes, R.M., et al., Human liver cell spheroids in extended perfusion bioreactor culture for repeated-dose drug testing. Hepatology, 2012. 55(4): $p$. 1227-36.

130. Kobayashi, A., et al., Regulation of differentiation and proliferation of rat hepatocytes by lactose-carrying polystyrene. Artif Organs, 1992. 16(6): p. 5647.

131. Drewitz, M., et al., Towards automated production and drug sensitivity testing using scaffold-free spherical tumor microtissues. Biotechnol J, 2011. 6(12): p. 1488-96.

132. Griner, L.M., et al., Generation of High-Throughput Three-Dimensional Tumor Spheroids for Drug Screening. J Vis Exp, 2018(139).

133. Fang, Y. and R.M. Eglen, Three-Dimensional Cell Cultures in Drug Discovery and Development. SLAS discovery : advancing life sciences R \& D, 2017. 22(5): p. 456-472.

134. Kim, M.S., et al., 3D tissue formation by stacking detachable cell sheets formed on nanofiber mesh. Biofabrication, 2017. 9(1): p. 015029. 
135. Kwon, O.H., et al., Rapid cell sheet detachment from poly $(\mathrm{N}$ isopropylacrylamide)-grafted porous cell culture membranes. J Biomed Mater Res, 2000. 50(1): p. 82-9.

136. Tsuda, Y., et al., The use of patterned dual thermoresponsive surfaces for the collective recovery as co-cultured cell sheets. Biomaterials, 2005. 26(14): p. 1885-93.

137. Kobayashi, J. and T. Okano, Fabrication of a thermoresponsive cell culture dish: a key technology for cell sheet tissue engineering. Science and technology of advanced materials, 2010. 11(1): p. 014111-014111.

138. Sasagawa, T., et al., Endothelial colony-forming cells for preparing prevascular three-dimensional cell-dense tissues using cell-sheet engineering. J Tissue Eng Regen Med, 2016. 10(9): p. 739-47.

139. Hakkinen, K.M., et al., Direct comparisons of the morphology, migration, cell adhesions, and actin cytoskeleton of fibroblasts in four different threedimensional extracellular matrices. Tissue Eng Part A, 2011. 17(5-6): p. 71324.

140. Szalowska, E., et al., Treatment of mouse liver slices with cholestatic hepatotoxicants results in down-regulation of Fxr and its target genes. BMC Med Genomics, 2013. 6: p. 39.

141. Vatakuti, S., et al., Validation of precision-cut liver slices to study drug-induced cholestasis: a transcriptomics approach. Arch Toxicol, 2017. 91(3): p. 14011412.

142. Olinga, P., et al., Comparison of five incubation systems for rat liver slices using functional and viability parameters. J Pharmacol Toxicol Methods, 1997. 38(2): p. 59-69.

143. de Graaf, I.A., et al., Preparation and incubation of precision-cut liver and intestinal slices for application in drug metabolism and toxicity studies. Nat Protoc, 2010. 5(9): p. 1540-51.

144. Leeman, W.R., I.A. van de Gevel, and A.A. Rutten, Cytotoxicity of retinoic acid, menadione and aflatoxin $B(1)$ in rat liver slices using Netwell inserts as a new culture system. Toxicol In Vitro, 1995. 9(3): p. 291-8.

145. Vickers, A.E., et al., Organ slice viability extended for pathway characterization: an in vitro model to investigate fibrosis. Toxicol Sci, 2004. 82(2): p. 534-44.

146. Olinga, P. and D. Schuppan, Precision-cut liver slices: a tool to model the liver ex vivo. J Hepatol, 2013. 58(6): p. 1252-3.

147. van de Bovenkamp, M., et al., Precision-cut liver slices as a new model to study toxicity-induced hepatic stellate cell activation in a physiologic milieu. Toxicol Sci, 2005. 85(1): p. 632-8. 
148. Shirahama, H., et al., Fabrication of Inverted Colloidal Crystal Poly(ethylene glycol) Scaffold: A Three-dimensional Cell Culture Platform for Liver Tissue Engineering. J Vis Exp, 2016(114).

149. Jiang, J., et al., Efficacy of Engineered Liver Tissue Based on Poly-L-lactic Acid Scaffolds and Fetal Mouse Liver Cells Cultured with Oncostatin M, Nicotinamide, and Dimethyl Sulfoxide. Tissue engineering, 2004. 10: p. 157786.

150. Grant, R., D.C. Hay, and A. Callanan, A Drug-Induced Hybrid Electrospun PolyCapro-Lactone: Cell-Derived Extracellular Matrix Scaffold for Liver Tissue Engineering. Tissue Eng Part A, 2017. 23(13-14): p. 650-662.

151. Rodriguez-Vazquez, M., et al., Chitosan and Its Potential Use as a Scaffold for Tissue Engineering in Regenerative Medicine. Biomed Res Int, 2015. 2015: p. 821279.

152. Bhattacharya, M., et al., Nanofibrillar cellulose hydrogel promotes threedimensional liver cell culture. J Control Release, 2012. 164(3): p. 291-8.

153. Dvir-Ginzberg, M., et al., Liver tissue engineering within alginate scaffolds: effects of cell-seeding density on hepatocyte viability, morphology, and function. Tissue Eng, 2003. 9(4): p. 757-66.

154. Mazza, G., et al., Rapid production of human liver scaffolds for functional tissue engineering by high shear stress oscillation-decellularization. Sci Rep, 2017. 7(1): p. 5534.

155. Jain, A. and R. Bansal, Applications of regenerative medicine in organ transplantation. Journal of pharmacy \& bioallied sciences, 2015. 7(3): p. 188194.

156. Mason, C. and P. Dunnill, A brief definition of regenerative medicine. Regen Med, 2008. 3(1): p. 1-5.

157. Benam, K.H., et al., Engineered in vitro disease models. Annu Rev Pathol, 2015. 10: p. 195-262.

158. Hunt, J.A., et al., Hydrogels for tissue engineering and regenerative medicine. Journal of Materials Chemistry B, 2014. 2(33): p. 5319-5338.

159. Niranjan, R., et al., A novel injectable temperature-sensitive zinc doped chitosan/beta-glycerophosphate hydrogel for bone tissue engineering. Int J Biol Macromol, 2013. 54: p. 24-9.

160. Martinez-Sanz, E., et al., Bone reservoir: Injectable hyaluronic acid hydrogel for minimal invasive bone augmentation. J Control Release, 2011. 152(2): p. 23240.

161. Jeon, J.E., et al., Dynamic compression improves biosynthesis of human zonal chondrocytes from osteoarthritis patients. Osteoarthritis Cartilage, 2012. 20(8): p. 906-15. 
162. Jin, R., et al., Enzyme-mediated fast in situ formation of hydrogels from dextrantyramine conjugates. Biomaterials, 2007. 28(18): p. 2791-800.

163. Hume, S.L., et al., Alignment of multi-layered muscle cells within threedimensional hydrogel macrochannels. Acta Biomater, 2012. 8(6): p. 2193-202.

164. Shinohara, S., et al., Fabrication of in vitro three-dimensional multilayered blood vessel model using human endothelial and smooth muscle cells and highstrength PEG hydrogel. J Biosci Bioeng, 2013. 116(2): p. 231-4.

165. Liu, Y. and M.B. Chan-Park, Hydrogel based on interpenetrating polymer networks of dextran and gelatin for vascular tissue engineering. Biomaterials, 2009. 30(2): p. 196-207.

166. Garbern, J.C., et al., Delivery of basic fibroblast growth factor with a $\mathrm{pH}$ responsive, injectable hydrogel to improve angiogenesis in infarcted myocardium. Biomaterials, 2011. 32(9): p. 2407-16.

167. Sabbah, H.N., et al., Augmentation of left ventricular wall thickness with alginate hydrogel implants improves left ventricular function and prevents progressive remodeling in dogs with chronic heart failure. JACC Heart Fail, 2013. 1(3): p. 252-8.

168. Gao, J., et al., The use of chitosan based hydrogel for enhancing the therapeutic benefits of adipose-derived MSCs for acute kidney injury. Biomaterials, 2012. 33(14): p. 3673-81.

169. Vasanthan, K.S., et al., Development of Porous Hydrogel Scaffolds with Multiple Cues for Liver Tissue Engineering. Regenerative Engineering and Translational Medicine, 2017. 3(3): p. 176-191.

170. Ye, S., et al., Hydrogels for Liver Tissue Engineering. 2019. 6(3): p. 59.

171. Tian, W.M., et al., Hyaluronic acid-poly-D-lysine-based three-dimensional hydrogel for traumatic brain injury. Tissue Eng, 2005. 11(3-4): p. 513-25.

172. Zhu, J. and R.E. Marchant, Design properties of hydrogel tissue-engineering scaffolds. Expert review of medical devices, 2011. 8(5): p. 607-626.

173. Jennings, J.A. and J.D. Bumgardner, Chitosan Based Biomaterials, Volume 2 - Tissue Engineering and Therapeutics. Elsevier.

174. Saldin, L.T., et al., Extracellular matrix hydrogels from decellularized tissues: Structure and function. Acta Biomater, 2017. 49: p. 1-15.

175. Sellaro, T.L., et al., Maintenance of human hepatocyte function in vitro by liverderived extracellular matrix gels. Tissue Eng Part A, 2010. 16(3): p. 1075-82.

176. Watt, F.M., Cell culture models of differentiation. Faseb j, 1991. 5(3): p. 28794. 
177. Lin, P., et al., Assessing porcine liver-derived biomatrix for hepatic tissue engineering. Tissue Eng, 2004. 10(7-8): p. 1046-53.

178. Kleinman, H.K. and G.R. Martin, Matrigel: basement membrane matrix with biological activity. Semin Cancer Biol, 2005. 15(5): p. 378-86.

179. Hughes, C.S., L.M. Postovit, and G.A. Lajoie, Matrigel: a complex protein mixture required for optimal growth of cell culture. Proteomics, 2010. 10(9): p. 1886-90.

180. LeCluyse, E., et al., Influence of extracellular matrix overlay and medium formulation on the induction of cytochrome $P-4502 B$ enzymes in primary cultures of rat hepatocytes. Drug Metab Dispos, 1999. 27(8): p. 909-15.

181. Talbot, N.C. and T.J. Caperna, Proteome array identification of bioactive soluble proteins/peptides in Matrigel: relevance to stem cell responses. Cytotechnology, 2015. 67(5): p. 873-83.

182. M. Ross, A., et al., Synthetic substrates for long-term stem cell culture. Polymer, 2012. 53: p. 2533-2539.

183. Badylak, S.F., Regenerative medicine and developmental biology: the role of the extracellular matrix. Anat Rec B New Anat, 2005. 287(1): p. 36-41.

184. Badylak, S.F., D.O. Freytes, and T.W. Gilbert, Extracellular matrix as a biological scaffold material: Structure and function. Acta Biomater, 2009. 5(1): p. 1-13.

185. Loneker, A.E., et al., Solubilized liver extracellular matrix maintains primary rat hepatocyte phenotype in-vitro. J Biomed Mater Res A, 2016. 104(7): p. 18467.

186. Coronado, R.E., et al., Decellularization and Solubilization of Porcine Liver for Use as a Substrate for Porcine Hepatocyte Culture: Method Optimization and Comparison. Cell Transplantation, 2017. 26(12): p. 1840-1854.

187. Tan, Q.W., et al., Hydrogel derived from decellularized porcine adipose tissue as a promising biomaterial for soft tissue augmentation. J Biomed Mater Res A, 2017. 105(6): p. 1756-1764.

188. Frantz, C., K.M. Stewart, and V.M. Weaver, The extracellular matrix at a glance. J Cell Sci, 2010. 123(Pt 24): p. 4195-200.

189. Skardal, A., et al., Tissue specific synthetic ECM hydrogels for 3-D in vitro maintenance of hepatocyte function. Biomaterials, 2012. 33(18): p. 4565-75.

190. Acikgöz, A., S. Giri, and A. Bader, Detection of nanolevel drug metabolites in an organotypic culture of primary human hepatocytes and porcine hepatocytes with special reference to a two-compartment model. International Journal of Nanomedicine, 2012. 7: p. 5859-5872. 
191. Klaas, M., et al., The alterations in the extracellular matrix composition guide the repair of damaged liver tissue. Sci Rep, 2016. 6: p. 27398.

192.

http://www. vivo.colostate.edu/hbooks/pathphys/digestion/liver/histo sin usoids.html.

193. Wang, J., et al., Reduction of hepatic fibrosis by overexpression of von HippelLindau protein in experimental models of chronic liver disease. Scientific Reports, 2017. 7: p. 41038.

194. Jiang, Y., et al., Click hydrogels, microgels and nanogels: emerging platforms for drug delivery and tissue engineering. Biomaterials, 2014. 35(18): p. 496985.

195. Li, Y., J. Rodrigues, and H. Tomas, Injectable and biodegradable hydrogels: gelation, biodegradation and biomedical applications. Chem Soc Rev, 2012. 41(6): p. 2193-221.

196. Schild, H.G., Poly(N-isopropylacrylamide): experiment, theory and application. Progress in Polymer Science, 1992. 17(2): p. 163-249.

197. Kaneko, Y., et al., Rapid Deswelling Response of Poly(N-isopropylacrylamide) Hydrogels by the Formation of Water Release Channels Using Poly(ethylene oxide) Graft Chains. Macromolecules, 1998. 31(18): p. 6099-6105.

198. Seetapan, N., et al., Linear viscoelasticity of thermoassociative chitosan-gpoly(N-isopropylacrylamide) copolymer. Rheologica Acta, 2006. 45: p. 10111018.

199. Ding, H., et al., Synthesis and characterization of temperature-responsive copolymer of PELGA modified poly(N-isopropylacrylamide). Polymer, 2006. 47: p. $1575-1583$.

200. L. Roach, B., et al., Agarose Hydrogel Characterization for Regenerative Medicine Applications: Focus on Engineering Cartilage. 2016. p. 258-273.

201. Nagase, K., et al., Preparation of thermoresponsive cationic copolymer brush surfaces and application of the surface to separation of biomolecules. Biomacromolecules, 2008. 9(4): p. 1340-7.

202. Patil, N.S., J.S. Dordick, and D.G. Rethwisch, Macroporous poly(sucrose acrylate) hydrogel for controlled release of macromolecules. Biomaterials, 1996. 17(24): p. 2343-50.

203. He, L., et al., Direct Synthesis of Controlled-Structure Primary Amine-Based Methacrylic Polymers by Living Radical Polymerization. Macromolecules, 2007. 40(13): p. 4429-4438.

204. Choi, J.S. and H.S. Yoo, Pluronic/chitosan hydrogels containing epidermal growth factor with wound-adhesive and photo-crosslinkable properties. J Biomed Mater Res A, 2010. 95(2): p. 564-73. 
205. Ibrahim, S., et al., Characterization of glycidyl methacrylate - crosslinked hyaluronan hydrogel scaffolds incorporating elastogenic hyaluronan oligomers. Acta biomaterialia, 2011. 7(2): p. 653-665.

206. Rouillard, A.D., et al., Methods for photocrosslinking alginate hydrogel scaffolds with high cell viability. Tissue Eng Part C Methods, 2011. 17(2): p. 173-9.

207. Lutolf, M.P., et al., Cell-Responsive Synthetic Hydrogels. Advanced Materials, 2003. 15(11): p. 888-892.

208. Tan, $\mathrm{H}$., et al., Injectable in situ forming biodegradable chitosan-hyaluronic acid based hydrogels for cartilage tissue engineering. Biomaterials, 2009. 30(13): p. 2499-506.

209. Schense, J.C. and J.A. Hubbell, Cross-linking exogenous bifunctional peptides into fibrin gels with factor XIIla. Bioconjug Chem, 1999. 10(1): p. 75-81.

210. Ryan, B.J., N. Carolan, and C. O'Fagain, Horseradish and soybean peroxidases: comparable tools for alternative niches? Trends Biotechnol, 2006. 24(8): p. 355-63.

211. Lee, K.Y. and D.J. Mooney, Alginate: properties and biomedical applications. Prog Polym Sci, 2012. 37(1): p. 106-126.

212. Patil, J., et al., lonotropic gelation and polyelectrolyte complexation: The novel techniques to design hydrogel particulate sustained, modulated drug delivery system: A review. Vol. 5. 2010.

213. Mazza, G., et al., Decellularized human liver as a natural 3D-scaffold for liver bioengineering and transplantation. Sci Rep, 2015. 5: p. 13079.

214. Fernandez-Cossio, S., et al., Biocompatibility of agarose gel as a dermal filler: histologic evaluation of subcutaneous implants. Plast Reconstr Surg, 2007. 120(5): p. 1161-9.

215. Hsu, M.J., et al., Long-Term In Vivo Monitoring of Adult-Derived Human Liver Stem/Progenitor Cells by Bioluminescence Imaging, Positron Emission Tomography, and Contrast-Enhanced Computed Tomography. Stem Cells Dev, 2017. 26(13): p. 986-1002.

216. Waldherr, M., et al., Use of HuH6 and other human-derived hepatoma lines for the detection of genotoxins: a new hope for laboratory animals? Archives of toxicology, 2018. 92(2): p. 921-934.

217. Schmittgen, T.D. and K.J. Livak, Analyzing real-time PCR data by the comparative $C(T)$ method. Nat Protoc, 2008. 3(6): p. 1101-8.

218. Lorvellec, M., et al., Mouse decellularised liver scaffold improves human embryonic and induced pluripotent stem cells differentiation into hepatocytelike cells. PLoS One, 2017. 12(12): p. e0189586. 
219. Loo Kiew, P. and M. Mat Don, The Influence of Acetic Acid Concentration on the Extractability of Collagen from the Skin of Hybrid Clarias sp. and Its Physicochemical Properties: A Preliminary Study. Vol. 2. 2013. 123-128.

220. Mavri-Damelin, D., et al., Ornithine transcarbamylase and arginase I deficiency are responsible for diminished urea cycle function in the human hepatoblastoma cell line HepG2. Int J Biochem Cell Biol, 2007. 39(3): p. 55564.

221. Platell, C., et al., The omentum. World journal of gastroenterology, 2000. 6(2): p. 169-176.

222. Mosala Nezhad, Z., et al., Small intestinal submucosa extracellular matrix (CorMatrix $(R))$ in cardiovascular surgery: a systematic review. Interact Cardiovasc Thorac Surg, 2016. 22(6): p. 839-50.

223. Hayano, T., et al., Primary structure of human pepsinogen $C$ gene. J Biol Chem, 1988. 263(3): p. 1382-5.

224. Ijima, H., et al., Physical Properties of the Extracellular Matrix of Decellularized Porcine Liver. Vol. 4. 2018. 39.

225. Colombo, S., et al., Normal liver stiffness and its determinants in healthy blood donors. Digestive and Liver Disease, 2011. 43(3): p. 231-236.

226. Baiocchini, A., et al., Extracellular Matrix Molecular Remodeling in Human Liver Fibrosis Evolution. PLOS ONE, 2016. 11(3): p. e0151736.

227. Skardal, A., et al., Substrate elasticity controls cell proliferation, surface marker expression and motile phenotype in amniotic fluid-derived stem cells. J Mech Behav Biomed Mater, 2013. 17: p. 307-16.

228. Dai, Z., et al., Sterilization techniques for biodegradable scaffolds in tissue engineering applications. J Tissue Eng, 2016. 7: p. 2041731416648810.

229. Griffin, M., et al., Evaluation of Sterilisation Techniques for Regenerative Medicine Scaffolds Fabricated with Polyurethane Nonbiodegradable and Bioabsorbable Nanocomposite Materials. Int J Biomater, 2018. 2018: p. 6565783.

230. $\mathrm{Hu}, \mathrm{C}$. and $\mathrm{L}$. Li, In vitro culture of isolated primary hepatocytes and stem cellderived hepatocyte-like cells for liver regeneration. Protein Cell, 2015. 6(8): p. 562-74.

231. Luckert, C., et al., Comparative analysis of $3 D$ culture methods on human HepG2 cells. Arch Toxicol, 2017. 91(1): p. 393-406.

232. Costa, E.C., et al., Spheroids Formation on Non-Adhesive Surfaces by Liquid Overlay Technique: Considerations and Practical Approaches. Biotechnol J, 2018. 13(1). 
233. Jang, M., et al., On-chip three-dimensional cell culture in phaseguides improves hepatocyte functions in vitro. Biomicrofluidics, 2015. 9(3): p. 034113.

234. Ebert, A.D., P. Liang, and J.C. Wu, Induced pluripotent stem cells as a disease modeling and drug screening platform. J Cardiovasc Pharmacol, 2012. 60(4): p. 408-16.

235. Li, Y., et al., Patient-Specific Induced Pluripotent Stem Cell-Based Disease Model for Pathogenesis Studies and Clinical Pharmacotherapy. Circulation. Arrhythmia and electrophysiology, 2017. 10(6): p. e005398.

236. Hannan, N.R., et al., Production of hepatocyte-like cells from human pluripotent stem cells. Nat Protoc, 2013. 8(2): p. 430-7.

237. Sampaziotis, F., et al., Directed differentiation of human induced pluripotent stem cells into functional cholangiocyte-like cells. Nat Protoc, 2017. 12(4): $p$. 814-827.

238. Millman, J.R. and F.W. Pagliuca, Autologous Pluripotent Stem Cell-Derived beta-Like Cells for Diabetes Cellular Therapy. Diabetes, 2017. 66(5): p. 11111120.

239. Batalov, I. and A.W. Feinberg, Differentiation of Cardiomyocytes from Human Pluripotent Stem Cells Using Monolayer Culture. Biomark Insights, 2015. 10(Suppl 1): p. 71-6.

240. Salimi, A., et al., Comparison of different protocols for neural differentiation of human induced pluripotent stem cells. Mol Biol Rep, 2014. 41(3): p. 1713-21.

241. Wertheim, J.A. and J.R. Leventhal, Clinical implications of basic science discoveries: induced pluripotent stem cell therapy in transplantation--a potential role for immunologic tolerance. American journal of transplantation : official journal of the American Society of Transplantation and the American Society of Transplant Surgeons, 2015. 15(4): p. 887-890.

242. Nishikawa, S., R.A. Goldstein, and C.R. Nierras, The promise of human induced pluripotent stem cells for research and therapy. Nat Rev Mol Cell Biol, 2008. 9(9): p. 725-9.

243. Sauer, V., et al., Induced pluripotent stem cells as a source of hepatocytes. Current pathobiology reports, 2014. 2(1): p. 11-20.

244. Kruth, J.P., Material Incress Manufacturing by Rapid Prototyping Techniques. CIRP Annals, 1991. 40(2): p. 603-614.

245. Hull Charles, W., Apparatus For Production Of Three-dimensional Objects By Stereolithography. 1986, UVP INC: US.

246. Chia, H.N. and B.M. Wu, Recent advances in $3 D$ printing of biomaterials. Journal of biological engineering, 2015. 9: p. 4-4. 
247. Chimene, D., et al., Advanced Bioinks for 3D Printing: A Materials Science Perspective. Ann Biomed Eng, 2016. 44(6): p. 2090-102.

248. Ji, S. and M. Guvendiren, Recent Advances in Bioink Design for 3D Bioprinting of Tissues and Organs. Frontiers in bioengineering and biotechnology, 2017. 5: p. 23-23.

249. Gopinathan, J. and I. Noh, Recent trends in bioinks for 3D printing. Biomaterials research, 2018. 22: p. 11-11.

250. Chen, Y.W., et al., Osteogenic and angiogenic potentials of the cell-laden hydrogel/mussel-inspired calcium silicate complex hierarchical porous scaffold fabricated by 3D bioprinting. Mater Sci Eng C Mater Biol Appl, 2018. 91: p. 679687.

251. Kaye, R., et al., A 3-dimensional bioprinted tracheal segment implant pilot study: Rabbit tracheal resection with graft implantation. Int $\mathrm{J}$ Pediatr Otorhinolaryngol, 2019. 117: p. 175-178.

252. Jose, R.R., et al., Evolution of Bioinks and Additive Manufacturing Technologies for 3D Bioprinting. ACS Biomaterials Science \& Engineering, 2016. 2(10): p. 1662-1678.

253. Tappa, K. and U. Jammalamadaka, Novel Biomaterials Used in Medical 3D Printing Techniques. J Funct Biomater, 2018. 9(1).

254. Xiong, J.Y., et al., Topology evolution and gelation mechanism of agarose gel. J Phys Chem B, 2005. 109(12): p. 5638-43.

255. Fedorovich, N.E., et al., Three-dimensional fiber deposition of cell-laden, viable, patterned constructs for bone tissue printing. Tissue Eng Part A, 2008. 14(1): p. 127-33.

256. Kreimendahl, F., et al., Three-Dimensional Printing and Angiogenesis: Tailored Agarose-Type I Collagen Blends Comprise Three-Dimensional Printability and Angiogenesis Potential for Tissue-Engineered Substitutes. Tissue Eng Part C Methods, 2017. 23(10): p. 604-615.

257. Yang, X., et al., Collagen-alginate as bioink for three-dimensional (3D) cell printing based cartilage tissue engineering. Mater Sci Eng C Mater Biol Appl, 2018. 83: p. 195-201.

258. Inoue, A., Characterization of PL-7 Family Alginate Lyases From Marine Organisms and Their Applications. Methods Enzymol, 2018. 605: p. 499-524.

259. Ozbolat, I.T. and M. Hospodiuk, Current advances and future perspectives in extrusion-based bioprinting. Biomaterials, 2016. 76: p. 321-43.

260. Christensen, K., et al., Freeform inkjet printing of cellular structures with bifurcations. Biotechnol Bioeng, 2015. 112(5): p. 1047-55. 
261. Lawson, M.A., et al., Adhesion and growth of bone marrow stromal cells on modified alginate hydrogels. Tissue Eng, 2004. 10(9-10): p. 1480-91.

262. Ferreira, A.M., et al., Collagen for bone tissue regeneration. Acta Biomater, 2012. 8(9): p. 3191-200.

263. Grzesik, W.J. and P.G. Robey, Bone matrix RGD glycoproteins: immunolocalization and interaction with human primary osteoblastic bone cells in vitro. J Bone Miner Res, 1994. 9(4): p. 487-96.

264. Park, J.Y., et al., A comparative study on collagen type I and hyaluronic acid dependent cell behavior for osteochondral tissue bioprinting. Biofabrication, 2014. 6(3): p. 035004.

265. Homenick, C.M., et al., Pluronics as crosslinking agents for collagen: novel amphiphilic hydrogels. Polymer International, 2011. 60(3): p. 458-465.

266. Yoo, H.S., et al., Hyaluronic acid modified biodegradable scaffolds for cartilage tissue engineering. Biomaterials, 2005. 26(14): p. 1925-33.

267. Jeon, O., et al., Mechanical properties and degradation behaviors of hyaluronic acid hydrogels cross-linked at various cross-linking densities. Carbohydrate Polymers, 2007. 70(3): p. 251-257.

268. Gerecht, S., et al., Hyaluronic acid hydrogel for controlled self-renewal and differentiation of human embryonic stem cells. Proc Natl Acad Sci U S A, 2007. 104(27): p. 11298-303.

269. Piras, C.C., S. Fernandez-Prieto, and W.M. De Borggraeve, Nanocellulosic materials as bioinks for 3D bioprinting. Biomater Sci, 2017. 5(10): p. 1988-1992.

270. Markstedt, K., et al., 3D Bioprinting Human Chondrocytes with NanocelluloseAlginate Bioink for Cartilage Tissue Engineering Applications. Biomacromolecules, 2015. 16(5): p. 1489-96.

271. Nguyen, D., et al., Cartilage Tissue Engineering by the 3D Bioprinting of iPS Cells in a Nanocellulose/Alginate Bioink. Sci Rep, 2017. 7(1): p. 658.

272. Guvendiren, M. and J.A. Burdick, Engineering synthetic hydrogel microenvironments to instruct stem cells. Curr Opin Biotechnol, 2013. 24(5): p. 841-6.

273. Mozetic, P., et al., Engineering muscle cell alignment through 3D bioprinting. J Biomed Mater Res A, 2017. 105(9): p. 2582-2588.

274. Cui, X., et al., Direct human cartilage repair using three-dimensional bioprinting technology. Tissue Eng Part A, 2012. 18(11-12): p. 1304-12.

275. Mandrycky, C., et al., 3D bioprinting for engineering complex tissues. Biotechnol Adv, 2016. 34(4): p. 422-434. 
276. Chan, B.P. and K.W. Leong, Scaffolding in tissue engineering: general approaches and tissue-specific considerations. European spine journal : official publication of the European Spine Society, the European Spinal Deformity Society, and the European Section of the Cervical Spine Research Society, 2008. 17 Suppl 4(Suppl 4): p. 467-479.

277. Kabirian, F. and M. Mozafari, Decellularized ECM-derived bioinks: Prospects for the future. Methods, 2019.

278. Pati, F., et al., Printing three-dimensional tissue analogues with decellularized extracellular matrix bioink. Nature Communications, 2014. 5: p. 3935.

279. Kim, H., et al., Characterization of cornea-specific bioink: high transparency, improved in vivo safety. J Tissue Eng, 2019. 10: p. 2041731418823382.

280. Kim, B.S., et al., 3D cell printing of in vitro stabilized skin model and in vivo prevascularized skin patch using tissue-specific extracellular matrix bioink: A step towards advanced skin tissue engineering. Biomaterials, 2018. 168: p. 38-53.

281. Weiskirchen, R., et al., Genetic Characteristics of the Human Hepatic Stellate Cell Line LX-2. PLOS ONE, 2013. 8(10): p. e75692.

282. Fedorovich, N.E., et al., Hydrogels as extracellular matrices for skeletal tissue engineering: state-of-the-art and novel application in organ printing. Tissue Eng, 2007. 13(8): p. 1905-25.

283. Blaeser, A., et al., Controlling Shear Stress in 3D Bioprinting is a Key Factor to Balance Printing Resolution and Stem Cell Integrity. Adv Healthc Mater, 2016. 5(3): p. 326-33.

284. Halliday, P., et al., Preparation of the porcine liver for allotransplantation. Aust N Z J Surg, 1971. 40(4): p. 374-80.

285. Porzionato, A., et al., Tissue-Engineered Grafts from Human Decellularized Extracellular Matrices: A Systematic Review and Future Perspectives. International journal of molecular sciences, 2018. 19(12): p. 4117.

286. Koci, Z., et al., Extracellular Matrix Hydrogel Derived from Human Umbilical Cord as a Scaffold for Neural Tissue Repair and Its Comparison with Extracellular Matrix from Porcine Tissues. Tissue Eng Part C Methods, 2017. 23(6): p. 333-345.

287. Gomez-Lechon, M.J., J.V. Castell, and M.T. Donato, Hepatocytes--the choice to investigate drug metabolism and toxicity in man: in vitro variability as a reflection of in vivo. Chem Biol Interact, 2007. 168(1): p. 30-50.

288. Gaca, M.D., et al., Basement membrane-like matrix inhibits proliferation and collagen synthesis by activated rat hepatic stellate cells: evidence for matrixdependent deactivation of stellate cells. Matrix Biol, 2003. 22(3): p. 229-39. 
289. Prestigiacomo, V., et al., Pro-fibrotic compounds induce stellate cell activation, ECM-remodelling and Nrf2 activation in a human 3D-multicellular model of liver fibrosis. PLoS One, 2017. 12(6): p. e0179995.

290. Zhao, W., et al., Inhibition of lysyl oxidase-like 1 (LOXL1) expression arrests liver fibrosis progression in cirrhosis by reducing elastin crosslinking. Biochim Biophys Acta Mol Basis Dis, 2018. 1864(4 Pt A): p. 1129-1137.

291. Fabregat, I. and D. Caballero-Díaz, Transforming Growth Factor- $\beta$-Induced Cell Plasticity in Liver Fibrosis and Hepatocarcinogenesis. Frontiers in oncology, 2018. 8: p. 357-357.

292. Stanger, B.Z., Cellular homeostasis and repair in the mammalian liver. Annual review of physiology, 2015. 77: p. 179-200.

293. Yin, C., et al., Hepatic stellate cells in liver development, regeneration, and cancer. J Clin Invest, 2013. 123(5): p. 1902-10.

294. Murgia, M., et al., Controlling metabolism and cell death: at the heart of mitochondrial calcium signalling. Journal of molecular and cellular cardiology, 2009. 46(6): p. 781-788.

295. Paulsen, S.J. and J.S. Miller, Tissue vascularization through 3D printing: Will technology bring us flow? Dev Dyn, 2015. 244(5): p. 629-40.

296. Sarker, M.D., et al., 3D biofabrication of vascular networks for tissue regeneration: A report on recent advances. J Pharm Anal, 2018. 8(5): p. 277296.

297. Miri, A.K., et al., Effective bioprinting resolution in tissue model fabrication. Lab Chip, 2019. 19(11): p. 2019-2037.

298. Dhawan, A., et al., Hepatocyte transplantation for inherited factor VII deficiency. Transplantation, 2004. 78(12): p. 1812-4.

299. Rouwkema, J., et al., Supply of Nutrients to Cells in Engineered Tissues. Biotechnology and Genetic Engineering Reviews, 2009. 26(1): p. 163-178.

300. Williams, R., et al., Addressing liver disease in the UK: a blueprint for attaining excellence in health care and reducing premature mortality from lifestyle issues of excess consumption of alcohol, obesity, and viral hepatitis. Lancet, 2014. 384(9958): p. 1953-97.

301. Maddams, J., M. Utley, and H. Moller, Projections of cancer prevalence in the United Kingdom, 2010-2040. Br J Cancer, 2012. 107(7): p. 1195-202.

302. Jemal, A., et al., Global patterns of cancer incidence and mortality rates and trends. Cancer Epidemiol Biomarkers Prev, 2010. 19(8): p. 1893-907.

303. Graham Kristie, H.Y.K. World Cancer Declaration Progress Report 2016 / UICC. 2016. 
304. Ferlay, J., et al., Estimates of worldwide burden of cancer in 2008: GLOBOCAN 2008. Int J Cancer, 2010. 127(12): p. 2893-917.

305. Mittal, S. and H.B. El-Serag, Epidemiology of hepatocellular carcinoma: consider the population. J Clin Gastroenterol, 2013. 47 Suppl: p. S2-6.

306. British Association for the Study of the Liver (BASL), B.S.o.G.B.L.S., A TIME TO ACT:IMPROVING LIVER HEALTH AND OUTCOMES IN LIVER DISEASE 2009.

307. Bruix, J. and M. Sherman, Management of hepatocellular carcinoma: an update. Hepatology, 2011. 53(3): p. 1020-2.

308. Mikula, M., et al., Activated hepatic stellate cells induce tumor progression of neoplastic hepatocytes in a TGF-beta dependent fashion. J Cell Physiol, 2006. 209(2): p. 560-7.

309. Farazi, P.A. and R.A. DePinho, Hepatocellular carcinoma pathogenesis: from genes to environment. Nat Rev Cancer, 2006. 6(9): p. 674-87.

310. Golabi, P., et al., Mortality assessment of patients with hepatocellular carcinoma according to underlying disease and treatment modalities. Medicine (Baltimore), 2017. 96(9): p. e5904.

311. Alizadeh, A.A., et al., Toward understanding and exploiting tumor heterogeneity. Nat Med, 2015. 21(8): p. 846-53.

312. Woo, H.G., et al., Exploring genomic profiles of hepatocellular carcinoma. Molecular carcinogenesis, 2011. 50(4): p. 235-243.

313. Boyault, S., et al., Transcriptome classification of HCC is related to gene alterations and to new therapeutic targets. Hepatology, 2007. 45(1): p. 42-52.

314. Calderaro, J., et al., Histological subtypes of hepatocellular carcinoma are related to gene mutations and molecular tumour classification. J Hepatol, 2017. 67(4): p. 727-738.

315. Nowak, A.K., P.K. Chow, and M. Findlay, Systemic therapy for advanced hepatocellular carcinoma: a review. Eur J Cancer, 2004. 40(10): p. 1474-84.

316. Novo, E. and M. Parola, Redox mechanisms in hepatic chronic wound healing and fibrogenesis. Fibrogenesis Tissue Repair, 2008. 1(1): p. 5.

317. Svegliati Baroni, G., et al., Fibrogenic effect of oxidative stress on rat hepatic stellate cells. Hepatology, 1998. 27(3): p. 720-6.

318. Yu, L.X., Y. Ling, and H.Y. Wang, Role of nonresolving inflammation in hepatocellular carcinoma development and progression. NPJ Precis Oncol, 2018. 2(1): p. 6.

319. Friedman, S.L., Hepatic stellate cells: protean, multifunctional, and enigmatic cells of the liver. Physiol Rev, 2008. 88(1): p. 125-72. 
320. Schuppan, D. and Y.O. Kim, Evolving therapies for liver fibrosis. J Clin Invest, 2013. 123(5): p. 1887-901.

321. Capece, D., et al., The inflammatory microenvironment in hepatocellular carcinoma: a pivotal role for tumor-associated macrophages. Biomed Res Int, 2013. 2013: p. 187204.

322. Maeda, S., et al., IKK Keta couples hepatocyte death to cytokine-driven compensatory proliferation that promotes chemical hepatocarcinogenesis. Cell, 2005. 121(7): p. 977-90.

323. Arzumanyan, A., H.M. Reis, and M.A. Feitelson, Pathogenic mechanisms in $H B V$ - and HCV-associated hepatocellular carcinoma. Nat Rev Cancer, 2013. 13(2): p. 123-35.

324. Mazzocca, A., et al., Tumor-secreted lysophostatidic acid accelerates hepatocellular carcinoma progression by promoting differentiation of peritumoral fibroblasts in myofibroblasts. Hepatology, 2011. 54(3): p. 920-30.

325. Cornelius, A., et al., Endothelial Expression of Endocan Is Strongly Associated with Tumor Progression in Pituitary Adenoma. Brain Pathology, 2012. 22(6): p. 757-764.

326. Parola, M., F. Marra, and M. Pinzani, Myofibroblast - like cells and liver fibrogenesis: Emerging concepts in a rapidly moving scenario. Mol Aspects Med, 2008. 29(1-2): p. 58-66.

327. Carloni, V., T.V. Luong, and K. Rombouts, Hepatic stellate cells and extracellular matrix in hepatocellular carcinoma: more complicated than ever. Liver Int, 2014. 34(6): p. 834-43.

328. Hong-Jin Chen, M.-H.H., Fang-Gui Xu, Hao-Jun Xu, Jun-Jun She, Hong-Ping $\mathrm{Xia}$, Understanding the inflammation-cancer transformation in the development of primary liver cancer. Hepatoma Research, 2018. 4:29

329. Lau, W.Y., Future perspectives for hepatocellular carcinoma. HPB : the official journal of the International Hepato Pancreato Biliary Association, 2003. 5(4): p. 206-213.

330. Di Marco, V., et al., Sorafenib: from literature to clinical practice. Ann Oncol, 2013. 24 Suppl 2: p. ii30-7.

331. Mazzaferro, V., et al., Liver transplantation for the treatment of small hepatocellular carcinomas in patients with cirrhosis. N Engl J Med, 1996. 334(11): p. 693-9.

332. Zhu, Z., Milan criteria and its expansions in liver transplantation for hepatocellular carcinoma. Hepatobiliary surgery and nutrition, 2016. 5(6): p. 498-502. 
333. Llovet, J.M., J. Fuster, and J. Bruix, The Barcelona approach: diagnosis, staging, and treatment of hepatocellular carcinoma. Liver Transpl, 2004. 10(2 Suppl 1): p. S115-20.

334. Zamora-Valdes, D., T. Taner, and D.M. Nagorney, Surgical Treatment of Hepatocellular Carcinoma. Cancer control : journal of the Moffitt Cancer Center, 2017. 24(3): p. $1073274817729258-1073274817729258$.

335. Bilimoria, M.M., et al., Underlying liver disease, not tumor factors, predicts longterm survival after resection of hepatocellular carcinoma. Arch Surg, 2001. 136(5): p. 528-35.

336. Lee, G.C., et al., Surgical resection versus ablation for early-stage hepatocellular carcinoma: A retrospective cohort analysis. Am J Surg, 2019. 218(1): p. 157-163.

337. Riaz, A., et al., Radiologic-pathologic correlation of hepatocellular carcinoma treated with internal radiation using yttrium-90 microspheres. Hepatology, 2009. 49(4): p. 1185-93.

338. Llovet, J.M., et al., Arterial embolisation or chemoembolisation versus symptomatic treatment in patients with unresectable hepatocellular carcinoma: a randomised controlled trial. Lancet, 2002. 359(9319): p. 1734-9.

339. Wong, R. and C. Frenette, Updates in the management of hepatocellular carcinoma. Gastroenterol Hepatol (N Y), 2011. 7(1): p. 16-24.

340. McNamara, M.G., et al., Sorafenib as first-line therapy in patients with advanced Child-Pugh B hepatocellular carcinoma - a meta-analysis. European Journal of Cancer, 2018. 105: p. 1-9.

341. Liu, L., et al., Sorafenib blocks the RAF/MEK/ERK pathway, inhibits tumor angiogenesis, and induces tumor cell apoptosis in hepatocellular carcinoma model PLC/PRF/5. Cancer Res, 2006. 66(24): p. 11851-8.

342. Ben Mousa, A., Sorafenib in the treatment of advanced hepatocellular carcinoma. Saudi journal of gastroenterology : official journal of the Saudi Gastroenterology Association, 2008. 14(1): p. 40-42.

343. Llovet, J.R.S. and V. Mazzaferro, Sorafenib improves survival in advanced hepatocellular carcinoma (HCC): results of a phase III randomized placebocontrolled trial (SHARP trial). J Clin Oncol, 2007. 25.

344. Zhai, B., et al., Inhibition of Akt reverses the acquired resistance to sorafenib by switching protective autophagy to autophagic cell death in hepatocellular carcinoma. Mol Cancer Ther, 2014. 13(6): p. 1589-98.

345. Zhou, S.L., et al., Tumor-Associated Neutrophils Recruit Macrophages and TRegulatory Cells to Promote Progression of Hepatocellular Carcinoma and Resistance to Sorafenib. Gastroenterology, 2016. 150(7): p. 1646-1658.e17. 
346. Heo, Y.A. and Y.Y. Syed, Regorafenib: A Review in Hepatocellular Carcinoma. Drugs, 2018. 78(9): p. 951-958.

347. Kondo, M., et al., Treatment of Advanced Hepatocellular Carcinoma after Failure of Sorafenib Treatment: Subsequent or Additional Treatment Interventions Contribute to Prolonged Survival Postprogression. Gastroenterol Res Pract, 2017. 2017: p. 5728946.

348. Chen, J. and J. Gao, Advances in the study of molecularly targeted agents to treat hepatocellular carcinoma. Drug Discov Ther, 2014. 8(4): p. 154-64.

349. Cainap, C., et al., Linifanib versus Sorafenib in patients with advanced hepatocellular carcinoma: results of a randomized phase III trial. J Clin Oncol, 2015. 33(2): p. 172-9.

350. Zhu, A.X., et al., SEARCH: a phase III, randomized, double-blind, placebocontrolled trial of sorafenib plus erlotinib in patients with advanced hepatocellular carcinoma. J Clin Oncol, 2015. 33(6): p. 559-66.

351. Cheng, A.L., et al., Randomized, open-label phase 2 study comparing frontline dovitinib versus sorafenib in patients with advanced hepatocellular carcinoma. Hepatology, 2016. 64(3): p. 774-84.

352. Grunert, S., M. Jechlinger, and H. Beug, Diverse cellular and molecular mechanisms contribute to epithelial plasticity and metastasis. Nat Rev Mol Cell Biol, 2003. 4(8): p. 657-65.

353. Brabletz, T., To differentiate or not--routes towards metastasis. Nat Rev Cancer, 2012. 12(6): p. 425-36.

354. Nieto, M.A., Epithelial plasticity: a common theme in embryonic and cancer cells. Science, 2013. 342(6159): p. 1234850.

355. Xu, J., S. Lamouille, and R. Derynck, TGF-beta-induced epithelial to mesenchymal transition. Cell Res, 2009. 19(2): p. 156-72.

356. Osterreicher, C.H., et al., Fibroblast-specific protein 1 identifies an inflammatory subpopulation of macrophages in the liver. Proc Natl Acad Sci U S A, 2011. 108(1): p. 308-13.

357. Pinzani, M., Epithelial-mesenchymal transition in chronic liver disease: fibrogenesis or escape from death? J Hepatol, 2011. 55(2): p. 459-65.

358. Thiery, J.P., et al., Epithelial-mesenchymal transitions in development and disease. Cell, 2009. 139(5): p. 871-90.

359. van Zijl, F., G. Krupitza, and W. Mikulits, Initial steps of metastasis: cell invasion and endothelial transmigration. Mutat Res, 2011. 728(1-2): p. 23-34.

360. Papageorgis, P., TGF $\beta$ Signaling in Tumor Initiation, Epithelial-toMesenchymal Transition, and Metastasis. Journal of oncology, 2015. 2015: p. 587193-587193. 
361. Abdollah, S., et al., TbetaRI phosphorylation of Smad2 on Ser465 and Ser467 is required for Smad2-Smad4 complex formation and signaling. J Biol Chem, 1997. 272(44): p. 27678-85.

362. Xie, L., et al., Activation of the Erk pathway is required for TGF-beta1-induced EMT in vitro. Neoplasia (New York, N.Y.), 2004. 6(5): p. 603-610.

363. Zhang, Y.E., Non-Smad pathways in TGF-beta signaling. Cell Res, 2009. 19(1): p. 128-39.

364. Jian Hao, D.C., <strong $>$ TGF- $\beta$ signaling in hepatocellular carcinoma suppression and progression</strong>. 2018. 3(1): p. 10-21.

365. Cano, A., et al., The transcription factor snail controls epithelial-mesenchymal transitions by repressing E-cadherin expression. Nat Cell Biol, 2000. 2(2): p. 76-83.

366. Savagner, P., K.M. Yamada, and J.P. Thiery, The zinc-finger protein slug causes desmosome dissociation, an initial and necessary step for growth factor-induced epithelial-mesenchymal transition. The Journal of cell biology, 1997. 137(6): p. 1403-1419.

367. Eger, A., et al., DeltaEF1 is a transcriptional repressor of E-cadherin and regulates epithelial plasticity in breast cancer cells. Oncogene, 2005. 24(14): $p$. 2375-85.

368. Wendt, M.K., T.M. Allington, and W.P. Schiemann, Mechanisms of the epithelial-mesenchymal transition by TGF-beta. Future Oncol, 2009. 5(8): p. 1145-68.

369. Yang, J., et al., Twist, a master regulator of morphogenesis, plays an essential role in tumor metastasis. Cell, 2004. 117(7): p. 927-39.

370. Papageorgis, P., TGF\&\# $x 3 b 2$; Signaling in Tumor Initiation, Epithelial-toMesenchymal Transition, and Metastasis \%J Journal of Oncology. 2015. 2015: p. 15.

371. Tien, L.T., et al., Expression of beta-catenin in hepatocellular carcinoma. World J Gastroenterol, 2005. 11(16): p. 2398-401.

372. Amini Nik, S., et al., TGF-beta modulates beta-Catenin stability and signaling in mesenchymal proliferations. Exp Cell Res, 2007. 313(13): p. 2887-95.

373. Tian, X., et al., E-cadherin/beta-catenin complex and the epithelial barrier. J Biomed Biotechnol, 2011. 2011: p. 567305.

374. Zhang, S., et al., EPLIN downregulation promotes epithelial-mesenchymal transition in prostate cancer cells and correlates with clinical lymph node metastasis. Oncogene, 2011. 30(50): p. 4941-52.

375. Park, J.G., et al., Characterization of cell lines established from human hepatocellular carcinoma. Int J Cancer, 1995. 62(3): p. 276-82. 
376. Ku, J.-L. and J.-G. Park, Biology of SNU Cell Lines. Cancer Res Treat, 2005. 37(1): p. 1-19.

377. Kang, M.S., et al., Mutation of p53 gene in hepatocellular carcinoma cell lines with HBX DNA. Int J Cancer, 1996. 67(6): p. 898-902.

378. Kancherla, V., et al., Genomic Analysis Revealed New Oncogenic Signatures in TP53-Mutant Hepatocellular Carcinoma. Front Genet, 2018. 9: p. 2.

379. El-Kafrawy, S.A., et al., P53 mutations in hepatocellular carcinoma patients in Egypt. Int J Hyg Environ Health, 2005. 208(4): p. 263-70.

380. Weinberg, A.G. and M.J. Finegold, Primary hepatic tumors of childhood. Hum Pathol, 1983. 14(6): p. 512-37.

381. Lopez-Terrada, D., et al., Hep G2 is a hepatoblastoma-derived cell line. Hum Pathol, 2009. 40(10): p. 1512-5.

382. Shan, J., et al., High-Throughput Platform for Identifying Molecular Factors Involved in Phenotypic Stabilization of Primary Human Hepatocytes In Vitro. Journal of Biomolecular Screening, 2016. 21(9): p. 897-911.

383. Carpentier, A., et al., Hepatic differentiation of human pluripotent stem cells in miniaturized format suitable for high-throughput screen. Stem Cell Res, 2016. 16(3): p. 640-50.

384. Leenders, M.W., M.W. Nijkamp, and I.H. Borel Rinkes, Mouse models in liver cancer research: a review of current literature. World J Gastroenterol, 2008. 14(45): p. 6915-23.

385. Heindryckx, F., I. Colle, and H. Van Vlierberghe, Experimental mouse models for hepatocellular carcinoma research. Int J Exp Pathol, 2009. 90(4): p. 367-86.

386. Santos, N.P., A.A. Colaco, and P.A. Oliveira, Animal models as a tool in hepatocellular carcinoma research: A Review. Tumour Biol, 2017. 39(3): p. 1010428317695923.

387. Bakiri, L. and E.F. Wagner, Mouse models for liver cancer. Mol Oncol, 2013. 7(2): p. 206-23.

388. Magee, P.N. and J.M. Barnes, The production of malignant primary hepatic tumours in the rat by feeding dimethylnitrosamine. Br J Cancer, 1956. 10(1): $\mathrm{p}$. 114-22.

389. Dybing, E., et al., Metabolism and activation of 2-acetylaminofluorene in isolated rat hepatocytes. Cancer Res, 1979. 39(8): p. 3268-75.

390. Kang, J.S., et al., Role of CYP2E1 in thioacetamide-induced mouse hepatotoxicity. Toxicol Appl Pharmacol, 2008. 228(3): p. 295-300. 
391. McGlynn, K.A., et al., Susceptibility to aflatoxin B1-related primary hepatocellular carcinoma in mice and humans. Cancer Res, 2003. 63(15): p. 4594-601.

392. Kramer, M.G., et al., Evaluation of hepatocellular carcinoma models for preclinical studies. Drug Discovery Today: Disease Models, 2005. 2(1): p. 4149.

393. Cheon, D.J. and S. Orsulic, Mouse models of cancer. Annu Rev Pathol, 2011. 6: p. 95-119.

394. Shanks, N. and R. Greek, Experimental use of nonhuman primates is not a simple problem. Nat Med, 2008. 14(10): p. 1012; discussion 1012-3.

395. Hay, M., et al., Clinical development success rates for investigational drugs. Nat Biotechnol, 2014. 32(1): p. 40-51.

396. Arrowsmith, J., Trial watch: phase III and submission failures: 2007-2010. Nat Rev Drug Discov, 2011. 10(2): p. 87.

397. Astashkina, A., B. Mann, and D.W. Grainger, A critical evaluation of in vitro cell culture models for high-throughput drug screening and toxicity. Pharmacol Ther, 2012. 134(1): p. 82-106.

398. Bhatia, S.N. and D.E. Ingber, Microfluidic organs-on-chips. Nat Biotechnol, 2014. 32(8): p. 760-72.

399. Ronaldson-Bouchard, K. and G. Vunjak-Novakovic, Organs-on-a-Chip: A Fast Track for Engineered Human Tissues in Drug Development. Cell Stem Cell, 2018. 22(3): p. 310-324.

400. Satoh, T., et al., A multi-throughput multi-organ-on-a-chip system on a plate formatted pneumatic pressure-driven medium circulation platform. Lab on a Chip, 2018. 18(1): p. 115-125.

401. Menon, N.V., et al., A microfluidic co-culture system to monitor tumor-stromal interactions on a chip. Biomicrofluidics, 2014. 8(6): p. 064118.

402. Kern, M.A., et al., Ex vivo analysis of antineoplastic agents in precision-cut tissue slices of human origin: effects of cyclooxygenase-2 inhibition in hepatocellular carcinoma. Liver Int, 2006. 26(5): p. 604-12.

403. Alshareeda, A.T., et al., The potential of cell sheet technique on the development of hepatocellular carcinoma in rat models. PLOS ONE, 2017. 12(8): p. e0184004.

404. Fang, M., et al., In vitro invasive pattern of hepatocellular carcinoma cell line HCCLM9 based on three-dimensional cell culture and quantum dots molecular imaging. J Huazhong Univ Sci Technolog Med Sci, 2013. 33(4): p. 520-524. 
405. Molina-Jimenez, F., et al., Matrigel-embedded 3D culture of Huh-7 cells as a hepatocyte-like polarized system to study hepatitis $C$ virus cycle. Virology, 2012. 425(1): p. 31-9.

406. Fong, E.L.S., et al., Generation of matched patient-derived xenograft in vitro-in vivo models using $3 D$ macroporous hydrogels for the study of liver cancer. Biomaterials, 2018. 159: p. 229-240.

407. Leung, M., et al., Chitosan-alginate scaffold culture system for hepatocellular carcinoma increases malignancy and drug resistance. Pharmaceutical research, 2010. 27(9): p. 1939-1948.

408. Crapo, P.M., T.W. Gilbert, and S.F. Badylak, An overview of tissue and whole organ decellularization processes. Biomaterials, 2011. 32(12): p. 3233-43.

409. Blanazs, A., et al., Sterilizable Gels from Thermoresponsive Block Copolymer Worms. Journal of the American Chemical Society, 2012. 134(23): p. 97419748.

410. Trzpis, M., et al., Epithelial cell adhesion molecule: more than a carcinoma marker and adhesion molecule. Am J Pathol, 2007. 171(2): p. 386-95.

411. Gauthier, A. and M. Ho, Role of sorafenib in the treatment of advanced hepatocellular carcinoma: An update. Hepatol Res, 2013. 43(2): p. 147-54.

412. Zhang, X.D., et al., Hypoxia promotes epithelial-mesenchymal transition of hepatocellular carcinoma cells via inducing Twist1 expression. Eur Rev Med Pharmacol Sci, 2017. 21(13): p. 3061-3068.

413. Yamada, S., et al., Epithelial to mesenchymal transition is associated with shorter disease-free survival in hepatocellular carcinoma. Ann Surg Oncol, 2014. 21(12): p. 3882-90.

414. Gilbert, P.M., et al., Substrate elasticity regulates skeletal muscle stem cell selfrenewal in culture. Science, 2010. 329(5995): p. 1078-81.

415. Fung, J., et al., Defining Normal Liver Stiffness Range in a Normal Healthy Chinese Population without Liver Disease. PLOS ONE, 2013. 8(12): p. e85067.

416. Trédan, O., et al., Drug Resistance and the Solid Tumor Microenvironment. JNCl: Journal of the National Cancer Institute, 2007. 99(19): p. 1441-1454.

417. Fischbach, C., et al., Engineering tumors with 3D scaffolds. Nat Methods, 2007. 4(10): p. 855-60.

418. Smalley, K.S., M. Lioni, and M. Herlyn, Life isn't flat: taking cancer biology to the next dimension. In Vitro Cell Dev Biol Anim, 2006. 42(8-9): p. 242-7.

419. Xu, F. and K.J. Burg, Three-dimensional polymeric systems for cancer cell studies. Cytotechnology, 2007. 54(3): p. 135-43. 
420. Tredan, O., et al., Drug resistance and the solid tumor microenvironment. J Natl Cancer Inst, 2007. 99(19): p. 1441-54.

421. DiMasi, J.A. and H.G. Grabowski, Economics of new oncology drug development. J Clin Oncol, 2007. 25(2): p. 209-16.

422. Llovet, J.M., et al., Sorafenib in advanced hepatocellular carcinoma. N Engl J Med, 2008. 359(4): p. 378-90.

423. Pasqualato, A., et al., Quantitative shape analysis of chemoresistant colon cancer cells: correlation between morphotype and phenotype. Exp Cell Res, 2012. 318(7): p. 835-46.

424. Ayu Puspita, N. and A. Bedford, Morphological Changes of Cisplatin-resistant Human Breast Cancer MCF-7 Cell Line. International Journal of Integrated Health Sciences, 2017. 5: p. 8-14.

425. Pawaskar, D.K., et al., Physiologically based pharmacokinetic models for everolimus and sorafenib in mice. Cancer Chemother Pharmacol, 2013. 71(5): p. 1219-29.

426. Zheng, N., et al., Co-delivery of sorafenib and metapristone encapsulated by CXCR4-targeted PLGA-PEG nanoparticles overcomes hepatocellular carcinoma resistance to sorafenib. J Exp Clin Cancer Res, 2019. 38(1): p. 232.

427. Marin, J.J.G., et al., Mechanisms of Anticancer Drug Resistance in Hepatoblastoma. Cancers (Basel), 2019. 11(3).

428. Zhu, Y.J., et al., New knowledge of the mechanisms of sorafenib resistance in liver cancer. Acta Pharmacol Sin, 2017. 38(5): p. 614-622.

429. Zakaria, Y., et al., Eurycomanone induce apoptosis in HepG2 cells via upregulation of p53. Cancer Cell Int, 2009. 9: p. 16.

430. Wang, X.H., et al., TGF-beta1 signaling pathway serves a role in HepG2 cell regulation by affecting the protein expression of PCNA, gankyrin, $p 115, X I A P$ and survivin. Oncol Lett, 2017. 13(5): p. 3239-3246.

431. Sun, T., et al., Expression and functional significance of Twist1 in hepatocellular carcinoma: its role in vasculogenic mimicry. Hepatology, 2010. 51(2): p. 54556. 